\title{
PALLADIUM NANOPARTICLES ENCAPSULATED WITH RESVERATROL-DERIVED PHENOLS AND POLYPHENOLS FOR TARGETED PROSTATE CANCER THERAPY
}

\author{
A Dissertation \\ presented to \\ the Faculty of the Graduate School \\ at the University of Missouri-Columbia \\ In Partial Fulfillment \\ of the Requirements for the Degree \\ Doctor of Philosophy \\ by \\ VELAPHI CLEMENT THIPE
}

Professor. Kattesh V. Katti, Dissertation Supervisor

MAY 2019 
(C) Copyright by Velaphi C. Thipe 2019

All Rights Reserved 
The undersigned, appointed by the Associate Vice Chancellor of the Office of Research and Graduate School, have examined the dissertation entitled

\section{PALLADIUM NANOPARTICLES ENCAPSULATED WITH RESVERATROL- DERIVED PHENOLS AND POLYPHENOLS FOR TARGETED PROSTATE CANCER THERAPY}

Presented by Velaphi Clement Thipe

A candidate for the degree of Doctor of Philosophy

And hereby certify that, in their opinion, it is worthy of acceptance.

Professor. Kattesh V. Katti

Professor. Silvia S. Jurisson

Professor. Kent S. Gates

Professor. C. Michael Greenlief 


\section{DEDICATION}

This dissertation is dedicated to my late father and sister, James Phiri and Palesa Lucretia Thipe, respectively and my dearest mother, Shadi Patricia Thipe, who has been my pillar of strength throughout all my ups and downs. I would have not been able to reach for my dreams if not for their constant love, support and encouragement. You have stood by me no matter what and I am truly thankful for having you in my life. 


\section{ACKNOWLEDGEMENTS}

I would like to take this opportunity to first and foremost thank God for being my strength and guide throughout this journey. I would not have had the wisdom or the physical ability to accomplish this milestone in my life. The following people have played an enormous role in providing guidance, support and brainstorming throughout my doctoral studies:

I express my sincere gratitude to my advisor Prof. Kattesh V. Katti for devoting constant time and providing me with the opportunity to complete my Ph.D. degree. His vast knowledge and expertise in the field of green nanotechnology and nanomedicine for the development of nextgeneration cancer therapy enabled him to give me the right guidance and provided me with much needed motivation that made my Ph.D. experience productive and stimulating. I appreciate the opportunity to work on the project that I like. To my chair, Prof. Silvia S. Jurisson, who trained me in all aspects of my research toward my $\mathrm{PhD}$ I would like to thank her. Furthermore, I express my gratitude to my Graduate committee members (Prof. Katti, Prof. Jurrisson, Prof. C. Michael Greenlief and Prof. Kent S. Gates) for their guidance. I would like to thank Dr. Menka Khoobchandani for her constant advice and help throughout my work at Institute of Green NanoTechnology at MU. I really appreciate her teaching me a lot. I am very grateful to Mrs. Kavita Katti and Dr. Alice Raphael Karikachery for their assistance and advice with my experiments. They always guided, encouraged and helped me with my research work and writing my dissertation.

The electron microscopy core (EMCore) facility directed by Dr. Tommi White and her colleagues (DeAna Grant, Xiaoqing He and Martin Schauflinger), I would like to thank them for providing training, necessary facilitates for the characterization of palladium nanoparticles. I am very thankful to Lisa D. Watkinson, Terry Carmack, and Charles J. Smith (Harry S. Truman 
Memorial VA Hospital) for their valuable help with animal studies of Res-PdNPs. I would also like to thank Prof. Jurisson's radiopharmaceutical research group for giving me their valuable support, guidance and advice. I would also like to extend my gratitude to Dr. Brian Mooney his guidance, teaching, expertise and for conducting LC-MS analysis.

To the members of the University community who have become my friends, you have all been an inspiration and encouragement and have become like family to me. Most specifically I would like to thank the MU International Office (Jillian Collins, Lauren Pate, James Scott, David Currey, Dottie Heibel and other staff members); the MU African Interdisciplinary Studies Hub (Jenifer Pilz and Nadege Uwase) for their continuous support. On a personal level, I give thanks to my dear friends, Dr. Siphokazi Ntetha, Evonnia Woods, Olha Kholod, George Chingarande, Roxanne Wan, Pierce Bloebaum and his family, Shireen Mentor, Brian Chihera, Noor AzizanGardner, Nishant Jain, Kris Majumdar, Ann and Carl Korschgen, members of the Fulbright Student Organization, Missouri International Student Council and the African Graduate and Professional Student Association for their never-ending support, friendship and advice.

Most importantly, I would like to thank my family, they have supported me emotionally throughout my studies and who have always encouraged me. This journey was not easy due to the loss of my father and sister, it would not have been possible without the love and support of my dear mother (Shadi Patricia Thipe), who has been there throughout my studies and has always, encouraged me to pursue my dreams. I would not forget to give thanks to my nephews (Katleho and Kutwano Thipe) and nieces (Kamogetse and Rorisane Thipe) for giving me encouragement and understanding to never give up on my dreams. 
I would further like to acknowledge the following organizations and funding bodies that contributed tremendously towards the project:

- The Fulbright Student Exchange Program [Grant No. 15150089]

- The South African National Research Foundation (NRF): Nanobiotechnology for cancer therapy S\&F - Doctoral Abroad Grants [Grant No. 98141] 


\section{TABLE OF CONTENTS}

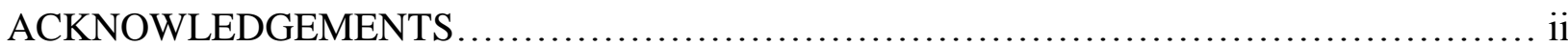

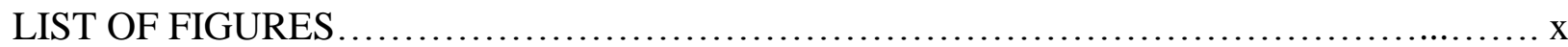

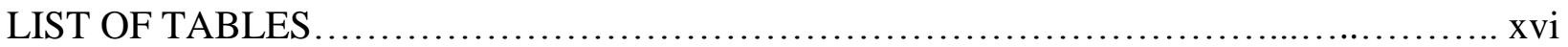

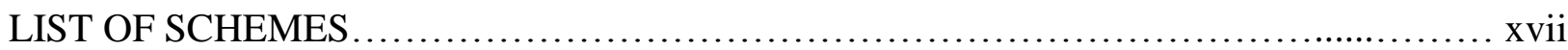

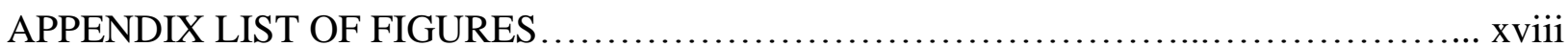

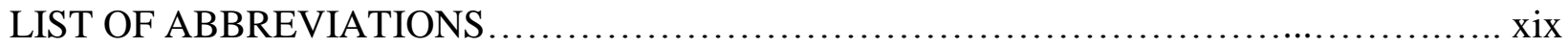

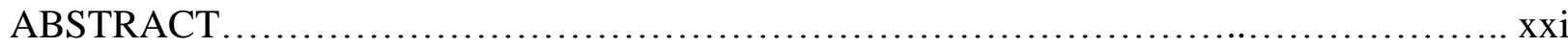

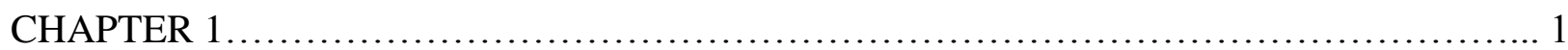

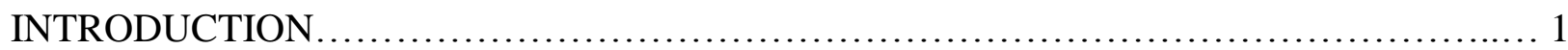

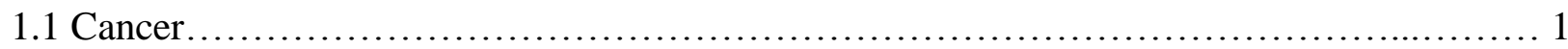

1.1.1 Prostate cancer.............................................................. 4

1.1.1.2 Types of prostate cancer................................................ 7

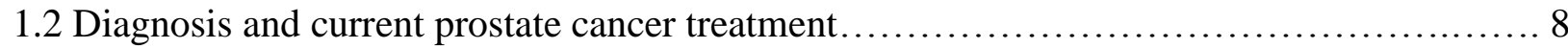

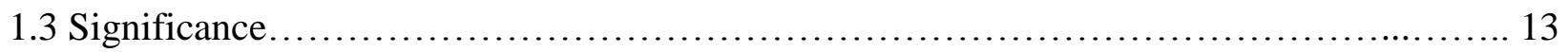

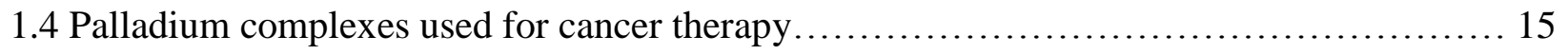

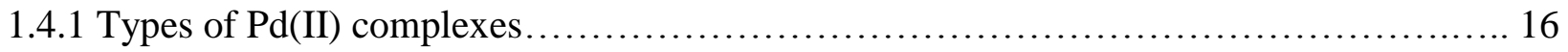

1.4.2 Challenges associated with $\mathrm{Pd}(\mathrm{II})$ complexes.................................. 16 
1.5 Plant-derived agents as anticancer pharmaceutical drugs for cancer therapy

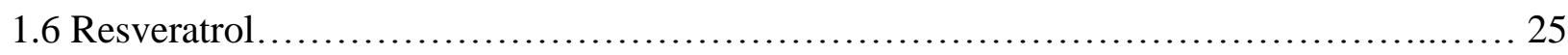

1.6.1 Anticancer mechanisms of resveratrol bioactivity............................. 28

1.6.1.1 Resveratrol delivery through various mode of administration..................... 33

1.6.2 Pitfalls and limitations associated with resveratrol delivery........................ 34

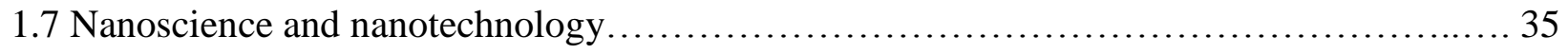

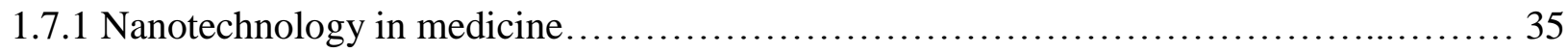

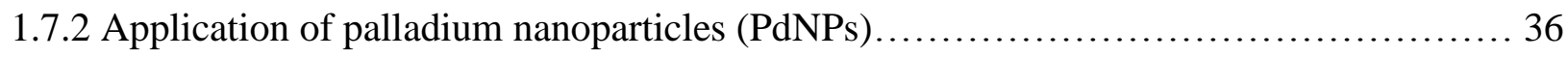

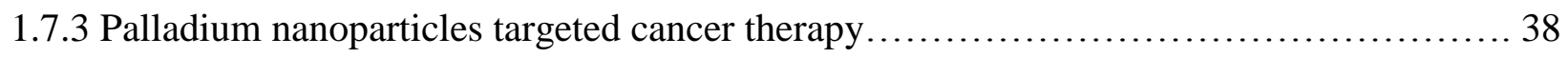

1.7.3.1 Toxicological effects of PdNPs............................................ 38

REFERENCES............................................................. 42

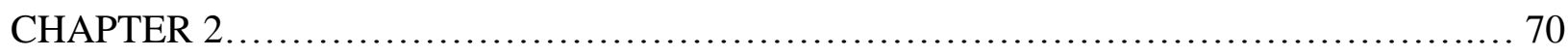

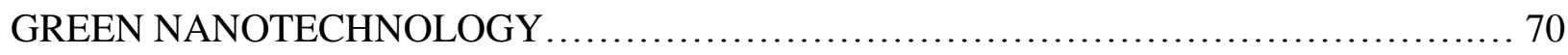

2.1 Green nanotechnology in cancer therapy - combinational modality.................... 70

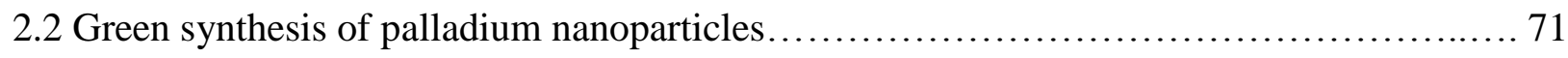

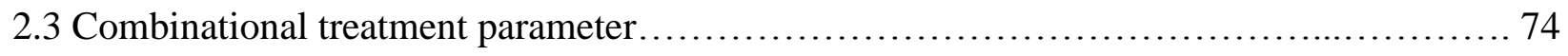

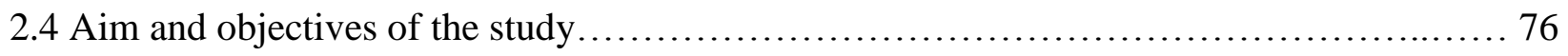

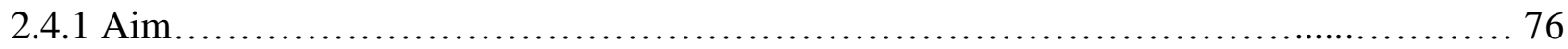




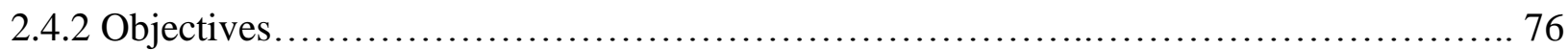

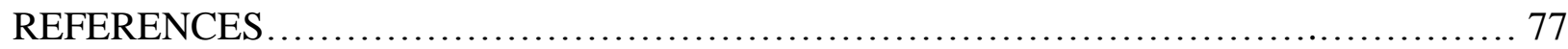

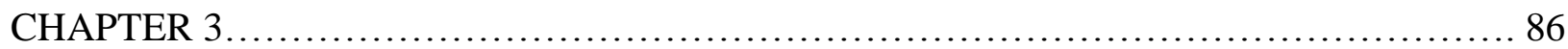

MATERIALS AND METHODS .......................................................... 86

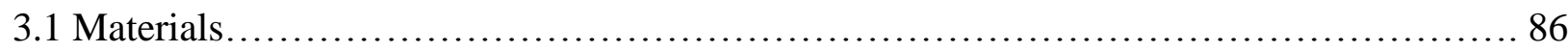

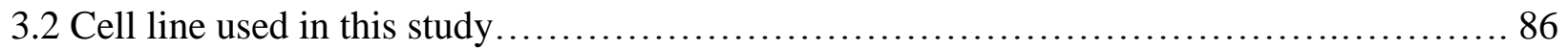

3.3 Characterization of palladium nanoparticles........................................ 87

3.4 Synthesis of resveratrol-derived phenols encapsulated palladium nanoparticles (Res-

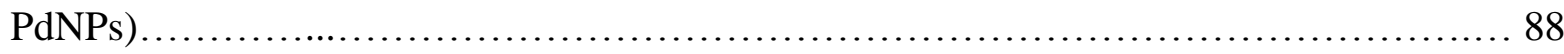

3.4.1 Res initiated and stabilized palladium nanoparticles (Res-PdNP-1 and Res-PdNP-2)..... 89

3.4.2 Res initiated and gum arabic stabilized palladium nanoparticles (Res-PdNP-3 and Res-

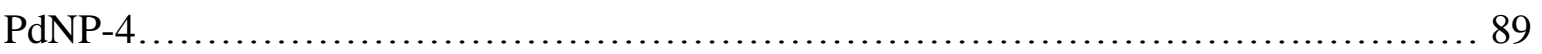

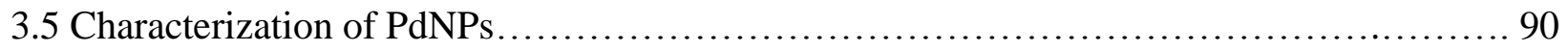

3.5.1 In vitro stability analysis of PdNPs.............................................. 90

3.5.2 Determination of palladium nanoparticles concentration.............................. 91

3.6 Quantification of resveratrol on the surface of PdNPs.................................. 95

3.6.1 Liquid chromatography-tandem mass spectrometry multiple reaction monitoring (LC-

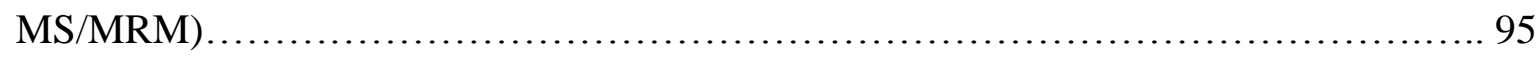

3.7 Cellular studies of PdNPs encapsulated with Res-derived phenols and polyphenols......... 96 


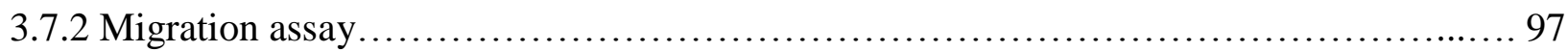

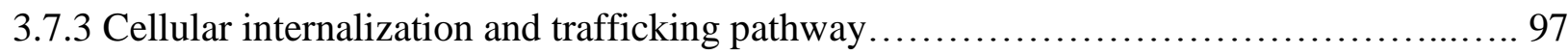

3.7.4 Confocal and dark-field microscopic technique..................................... 98

3.7.5 Transmission electron microscopy (TEM) technique.................................. 99

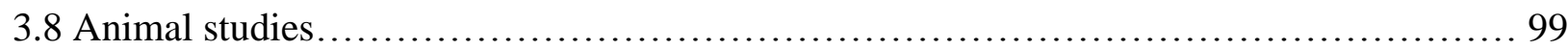

3.8.1 In vivo therapeutic efficacy study of Res-PdNPs................................... 100

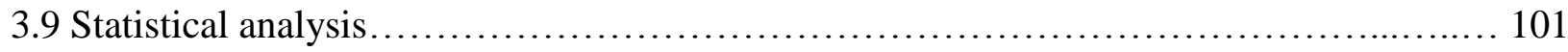

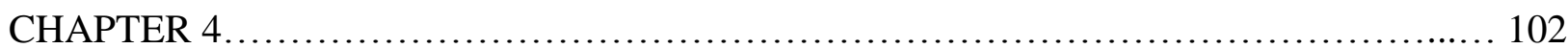

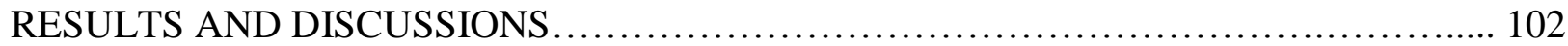

4.1 UV-Vis spectroscopy of palladium nanoparticles (PdNPs) encapsulated with resveratrol

(Res)-derived phenols and polyphenols............................................. 102

4.1.1 Res initiated and stabilized palladium nanoparticles (Res-PdNP-1 and Res-PdNP-2)... 102

4.1.2 Res initiated and Gum Arabic stabilized palladium nanoparticles (Res-PdNP-3 and Res-

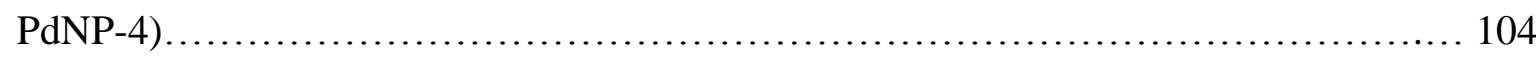

4.2 Electron microscopy studies of Res-PdNPs....................................... 106

4.3 Elemental analysis of Res-PdNPs.................................................. 114

4.4 In vitro stability of palladium nanoparticles....................................... 116

4.5 Fourier transform-infrared (FTIR) of Res-PdNP-3 and Res-PdNP-4 ................... 119 
4.6 Quantification of Res-derived phenols and polyphenolic molecules encapsulated onto the surface of the nanoparticles by liquid Chromatography-tandem mass spectrometry with

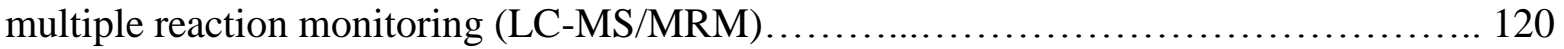

$4.7{ }^{1} \mathrm{H}$ nuclear magnetic resonance (NMR) analysis of the Res-PdNP-4................... 122

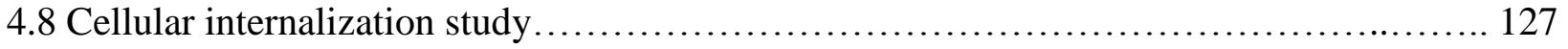

4.9 Receptor mediated cellular internalization of Res-PdNP-4 ......................... 132

4.10 Assessment of the cytotoxicity of Res-PdNP-4 against prostate cancer (PC-3) cells and

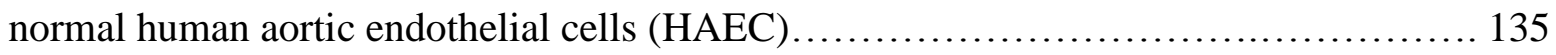

4.11 Cellular morphology and migration studies.................................. 138

4.12 Therapeutic efficacy study of Res-PdNP-4 ................................. 142

4.12.1 Immunohistochemistry of tumor xenografts................................. 146

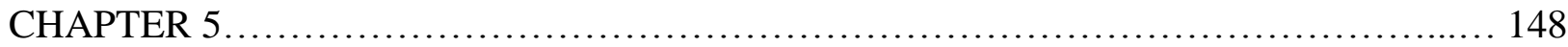

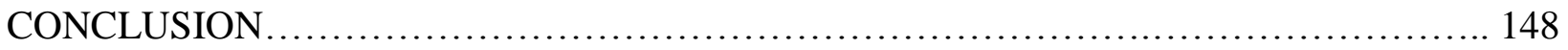

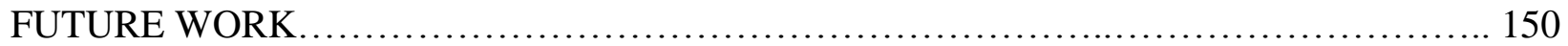

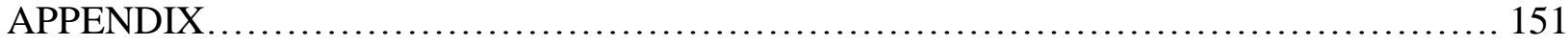

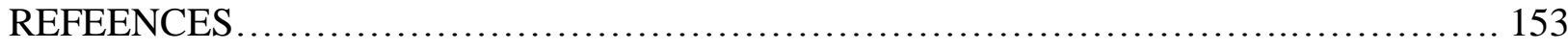

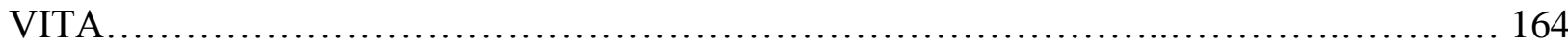




\section{LIST OF FIGURES}

Figure. Page

Figure 1: Cancer incidence worldwide in 2018. Breast, lung, prostate and colorectal cancers contribute a major public health burden across the globe. 2

Figure 2: Financial burden of cancer healthcare in the United States from 2010 to $2017 \ldots \ldots \ldots .3$

Figure 3: World map representing the most common type of cancer mortality in 2018 among men. Prostate cancer is ranked second after lung. Adapted with permission from

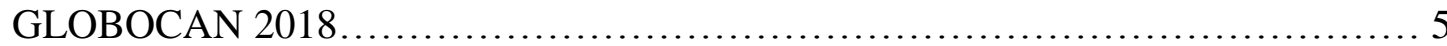

Figure 4: Prostate cancer tumorigenesis and metastasis from Stage I to Stage IV. The cancer cells grow within the prostate (Stage I and II), through the outer layer of the prostate into surrounding tissues (Stage III), and then to lymph nodes or other organs of the body (Stage IV).

Figure 5: Systemic therapy landscape of prostate cancer progression.

Figure 6: Platinum chemotherapeutic drugs used for the treatment of solid cancer (i) cisplatin,

(ii) carboplatin and (iii) oxaliplatin

Figure 7: Palladium complexes that have been investigated for their activity against tumor cells (i) trans- $\left[\mathrm{Pd}\{(\mathrm{R})-(+) \text {-bornylamine }\}_{2} \mathrm{Cl}_{2}\right.$, (ii) $\left[(\right.$ bipy $\left.) \mathrm{Pd}\left(\mathrm{SeO}_{3}\right)\right]$, (iii) $\mathrm{Pd}(\mathrm{II})$ complexes bearing 1,2-bis(diphenylphosphino)ethane (dppe), (iv) $\quad[($ Benzyl)Pd $\{$ bis(thiosemicar bazonate) $\}]$, and (v) [ trans- $\left.\mathrm{PtCl}\left(\mathrm{NH}_{3}\right)\right\} 2-\#-\left\{\right.$ trans- $\mathrm{Pd}\left(\mathrm{NH}_{3}\right)$ (2-hydroxypyridine)$\left.\left(\mathrm{H}_{2} \mathrm{~N}\left(\mathrm{CH}_{2}\right)_{6} \mathrm{NH}_{2}\right)_{2}\right]^{4+}$

Figure 8: Clinical benefit of resveratrol in cancer, cardiovascular diseases, chronic inflammation, 
Figure 9: Chemical structure of (i) 17- $\beta$ estradiol, (ii) Diethylstilbestrol, (iii) Resveratrol and (iv) Piceatannol, the red colored structure show the homology between these Compounds 28

Figure 10: Schematic diagram that depicts the cancer therapeutic mechanism of resveratrol, inducing apoptosis through p53-dependent activation of pro-apoptotic proteins (Bax, NOXA, and BUMA). Moreover, resveratrol upregulates Sirt1 and AMPK that induces cancer cell death by autophagy. The downregulation of the TGF- $\beta 1 / \mathrm{Smads}$, Wnt $/ \beta$ -

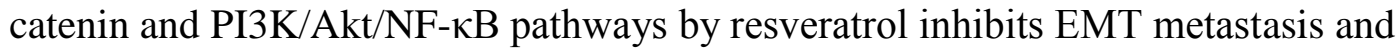
HIF-1 $\alpha /$ MMPs dependent inhibits VEGF angiogenesis.

Figure 11: A schematic representation of PdNPs photothermal therapy, where the external NIR laser excites the PdNPs to generate heat for hyperthermia.

Figure 12: Resveratrol A- and B-ring, and its molecular electrostatic potential (MEP), where the deep red color indicates an electron-rich site, whereas deep blue indicates an electrondeficient site 72

Figure 13: UV-Vis spectra of palladium nanoparticles encapsulated with resveratrol-derived phenols and polyphenols a) free Res, GA and Res-GA mixture, b) Res-PdNP-1 and Res-PdNP-2 and c) Res-PdNP-3 and Res-PdNP-4 .......................... 103

Figure 14: TEM image and size distribution of a) Res-PdNP-1 and b) Res-PdNP-2 ......... 107

Figure 15: TEM image and size distribution of a) Res-PdNP-3 and b) Res-PdNP-4.......... 108 Figure 16: HRTEM images of a) Res-PdNP-1, b) Res-PdNP-2 confirming the existence of individual Pd nanoclusters and c) schematic drawing visualizing Pd nanocluster 
agglomeration (structures are not drawn to scale)....

Figure 17: HRTEM images of a) Res-PdNP-3, b) Res-PdNP-4 crystalline nature and c)

Schematic drawing visualizing PdNPs nanocrystals (structures are not drawn to scale)

Figure 18: Crystal growth of palladium nanoparticles influenced by GA, and a schematic illustration of crystallization mechanism of amorphous palladium nanoparticles.... 114

Figure 19A: STEM-HAADF image and EDS elemental maps showing the presence of Pd, O, Cu, $\mathrm{Na}$ and $\mathrm{Cl}$ in a) Res-PdNP-1, b) Res-PdNP-2, respectively and c) EDS spectrum of Res-PdNPs 115

Figure 19B: STEM-HAADF image and EDS elemental maps showing the presence of Pd, O, Cu, $\mathrm{Si}, \mathrm{Na}$ and $\mathrm{Cl}$ in a) Res-PdNP-3, b) Res-PdNP-4 and c) EDS spectrum of ResPdNPs. 116

Figure 20: In vitro stability assay of Res-PdNPs by monitoring the hydrodynamic size of a) ResPdNP-1 and Res-PdNP-2 and b) Res-PdNP-3 and Res-PdNP-4 in various biological media at $37{ }^{\circ} \mathrm{C}$ for a week. Res-PdNP-1 and Res-PdNP-2 not stable in media (green

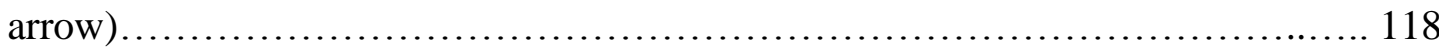

Figure 21: Fourier transform-infrared (FTIR) spectra of free Res and Res-PdNPs.... 120

Figure 22: LC-MS/MRM Quantitation of Res a) Res standard curve normalized to AUC for tolbutamide internal standard and b) Res-PdNPs monitored by MRM to specifically quantify the resveratrol in the samples..... 121

Figure 23: ${ }^{1} \mathrm{H}$ NMR spectra of a) Free Res, b) GA and c) Res-GA conjugate in $\mathrm{D}_{2} \mathrm{O} \ldots \ldots \ldots \ldots 123$

Figure 24: ${ }^{1} \mathrm{H}$ NMR spectra of Res-PdNP-4 in $\mathrm{D}_{2} \mathrm{O}$ (insert: magnified Res region)........... 124 
Figure 25: Chemical structure of resveratrol (a) and of its oxidation products piceatannol (b), 3,5dihydroxybenzoic acid (c), 3,5-dihydroxybenzaldehyde (d) and para-hydroxybenza ldehyde (e)

Figure 26: Mechanism for the formation of Res-PdNPs by using Res a) simplified mechanism of Res to quinone form with the reduction of $\mathrm{Pd} 2+$ to $\mathrm{Pd} 0$ by electron transfer and $b$ ) multiple oxidative products of Res 126

Figure 27: Dark field CytoViva images of prostate cancer (PC-3) cells showing cellular internalization post treatment a) control (no treatment), b) PC-3 cells incubated for 2 h, c) PC-3 cells incubated for $6 \mathrm{~h}$ and d) PC-3 cells incubated with $42 \mu \mathrm{g} / \mathrm{mL}$ ResPdNP-4 for $24 \mathrm{~h}$. 128

Figure 28: Confocal microscopy images of a) PC-3 control cells (no treatment) and b) 3D projection of a), c) PC-3 cells incubated with $42 \mu \mathrm{g} / \mathrm{mL}$ Res-PdNP-4 for $6 \mathrm{~h}$ and d) 3D projection of c). Nucleus (DAPI stain - blue), cytoplasm (WGA stain - green) and Res-PdNP-4 (yellow) 129

Figure 29: TEM images indicating cellular internalization of Res-PdNP-4 at $6 \mathrm{~h}$ post treatment a) PC-3 control cells (no treatment), b) PC-3 cells treated with $42 \mu \mathrm{g} / \mathrm{mL}$ Res-PdNP-4, c) magnified image of b) and d) size distribution histogram of Res-PdNP-4 ....... 130 Figure 30: TEM images indicating cellular internalization through endocytic pathway. $42 \mu \mathrm{g} / \mathrm{mL}$ Res-PdNP-4 at 6 h post treatment in PC-3 cancer cells. 131

Figure 31: Confocal microscopic images showing immunocytochemical localization of Lam 67R a) prostate cancer (PC-3) control cells (no treatment) and b) inhibition in Res-PdNP-4 uptake in the presence of Laminin 67 receptor blocking antibody (MLuC5) at $6 \mathrm{~h}$ post treatment in PC-3 cancer cells. 133 
Figure 32: STEM and EDS mapping of prostate cancer (PC-3) cells treated with $42 \mu \mathrm{g} / \mathrm{mL}$ ResPdNP-4 a) dark-field image of PC-3 cells; b) magnified image of a) showing a vaculoe with nanoparticles; c) EDS mapping of Res-PdNP-4 and d) EDS spectrum confirming the presence of Pd inside the prostate cancer cells

Figure 33: Cell viability of prostate cancer (PC-3) after $72 \mathrm{~h}$ post incubation with Res-PdNP-4 and controls (free Res, GA, GA-PdNPs, cisplatin and etoposide) 136

Figure 34: Cell viability of normal human aortic endothelial cells (HAEC) after $72 \mathrm{~h}$ post incubation with Res-PdNP-4 and controls (cisplatin and etoposide). At high ResPdNP-4 concentration no toxicity was observed (green arrow)

Figure 35: Cellular morphological studies of prostate cancer (PC-3) cells after $72 \mathrm{~h}$ post incubation with a) control (no treatment), b) cisplatin, c) etoposide and d) Res-PdNP4 for $72 \mathrm{~h}$ at $42 \mu \mathrm{g} / \mathrm{mL}$

Figure 36: Cellular morphological studies of normal human aortic endothelial cells (HAEC) after $72 \mathrm{~h}$ post incubation with a) control (no treatment), b) cisplatin, c) etoposide and d)

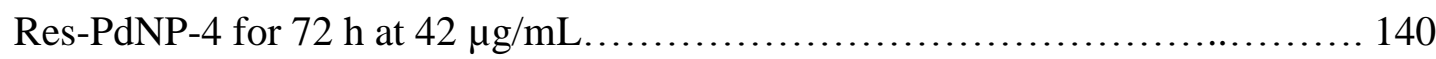

Figure 37: Cellular migration study of prostate cancer (PC-3) cells treated with Res-PdNP-4 compared with control cells (no treatment) 141

Figure 38: Therapeutic efficacy studies of prostate tumor-bearing SCID male mice treated by intravenous injection twice a week with Res-PdNP-4, Res and saline 144

Figure 39: Average body weight of the SCID male mice during the study..... 145

Figure 40: Immunohistochemical CD31 staining of tumor tissues to evaluate blood vessels.

Twelve fields from each xenograft were analyzed to determine the average number of vessels per field (microvessel density $=$ MVD). $\mathrm{N}=3$; mean \pm STEM; a) saline control xiv 
group, b) free Res treated group and c) Res-PdNP-4 treated group............. 147 


\section{LIST OF TABLES}

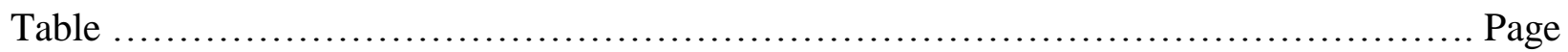

Table 1: Standard healthcare treatment for prostate cancer............................ 10

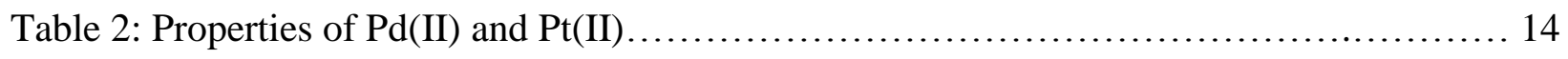

Table 3: Plant-derived drugs in the market used for the treatment of cancer and other-related

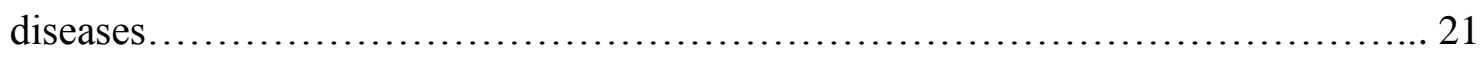

Table 4: Toxicology of palladium nanoparticles.................................. 39

Table 5: Antitumor study dose design for the animal body weight $(\mathrm{kg}) \ldots \ldots \ldots \ldots \ldots \ldots \ldots \ldots$

Table 6: Physiochemical properties of Res-PdNPs............................... 109

Table 7: In vitro cellular viability studies....................................... 138 


\section{LIST OF SCHEMES}

Scheme Page

Scheme 1: Reverse engineering and pharmacology of plant-derived anticancer drugs 1) food as a source of nutrients, 2) chemical extraction from plant material, 3) purification and identification of isolated compounds, 4) identification of active compound, 5) in-vitro cellular studies, 6) in-vivo anticancer pre-clinical studies, 7) bioinformatics and immunological studies and 8) clinical studies for prospective use as a cancer

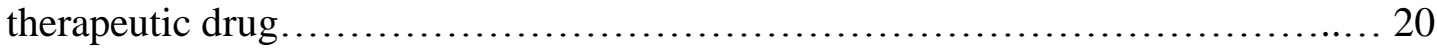

Scheme 2: Synthesis of PdNPs using resveratrol as a reducing and capping agent............ 88

Scheme 3: Unit cell of a crystalline palladium nanoparticle............................ 91 


\section{APPENDIX LIST OF FIGURES}

Figures ..... Page

Figure 41: Cell viability of prostate cancer (PC-3) after a) $24 \mathrm{~h}$ and b) $48 \mathrm{~h}$ post incubation with Res-PdNP-4 and controls (free Res, GA, GA-PdNPs, cisplatin and etoposide)....... 151

Figure 42: Cell viability of human aortic endothelial cells (HAEC) after a) $24 \mathrm{~h}$ and b) $48 \mathrm{~h}$ post incubation with Res-PdNP-4 and controls (cisplatin and etoposide). At high ResPdNP-4 concentration no toxicity was observed (green arrow). 152 


\section{LIST OF ABBREVIATIONS}

Abbreviation

BSA

DMSO

EDS

FBS

FT-IR

GA

GA-PdNPs

HAEC

HBSS

HSA

$\mathrm{IC}_{50}$

ICP-OES

LC-MS/MS

MRM

MTT

$\mathrm{NaBH}_{4}$

$\mathrm{NaCl}$

$\mathrm{Na}_{2} \mathrm{PdCl}_{4}$

NIR

MLuC5

PC-3
Definition

Bovine serum albumin

Dimethyl Sulfoxide Anhydrous

Energy-dispersive X-ray spectroscopy

Fetal Bovine Serum

Fourier-transform infrared spectroscopy

Gum arabic

Gum Arabic -palladium nanoparticles

Human aortic endothelial cells

Hank's Balanced Salt Solution

Human serum albumin

Half maximal inhibitory concentration

Inductively coupled plasma - optical emission spectrometry

Liquid chromatography-tandem mass spectrometry

Multiple reaction monitoring

(3-(4,5-dimethylthiazol-2-yl)-2,5-diphenyltetrazolium

bromide)

Sodium borohydride

Sodium chloride

Sodium tetrachloropalladate (II)

Near-infrared

RPSA monoclonal antibody

Prostate cancer cells 


$\begin{array}{ll}\text { Pd } & \text { Palladium } \\ \text { PFA } & \text { Paraformaldehyde } \\ \text { Pt } & \text { Platinum } \\ \text { Res } & \text { Resveratrol } \\ \text { Res-PdNP-1 } & \text { Resveratrol-palladium nanoparticles } \\ \text { Res-PdNP-2 } & \text { 3x Resveratrol-palladium nanoparticles } \\ \text { Res-PdNP-3 } & \text { Resveratrol gum arabic-palladium nanoparticles } \\ \text { Res-PdNP-4 } & \text { 3x Resveratrol gum arabic-palladium nanoparticles } \\ \text { RPMI } & \text { Roswell Park Memorial Institute Medium } \\ \text { SCID } & \text { Severely compromised immunodeficiency } \\ \text { TEM } & \text { Transmission electron microscopy } \\ \text { UV-vis } & \text { Ultraviolet-visible spectrophotometry } \\ 67 L R & \text { Laminin receptor }\end{array}$




\begin{abstract}
The main goal of this dissertation was to explore the development of a new generation of green nanoformulations through the production of biocompatible palladium nanoparticles using resveratrol to treat, image and evaluate the efficacy of the formulations in prostate cancer cells with minimal toxicity to surrounding normal tissues.
\end{abstract}

This dissertation is classified into three parts with three main objectives of the producing and characterizing resveratrol-derived phenols and polyphenols encapsulated palladium nanoparticles (Res-PdNPs) for the imaging and treatment of prostate cancer. Rigorous studies were performed for the optimization of the synthesis to achieve increased resveratrol-derived phenols and polyphenols corona loading on the palladium nanoparticle surface capable of providing adjuvant therapeutic benefits through delivering potent doses of both resveratrol phenols and nanoparticles directly to prostate cancer cells. A total of four formulations were produced ResPdNP-1 (resveratrol-palladium nanoparticles), Res-PdNP-2 (increased resveratrol corona loaded palladium nanoparticles), Res-PdNP-3 (resveratrol-gum arabic stabilized palladium nanoparticles) and Res-PdNP-4 (increased resveratrol corona loaded and compacted with gum arabic stabilized palladium nanoparticles), respectively. Electron microscopic (TEM) results revealed that role of gum arabic was not limited to the stability of the nanoparticles but also facilitated the crystallization of the produced palladium nanoparticles (Res-PdNP-3 and ResPdNP-4) and subsequently provided a supportive matrix for increased resveratrol phenols loading capacity. In vitro evaluation of the Res-PdNPs showed that Res-PdNP-1 and Res-PdNP2, were not stable in serum while Res-PdNP-3 and Res-PdNP-4 maintained superior stability, thus ruling out further analysis using Res-PdNP-1 and Res-PdNP-2. The LC-MS/MRM results 
confirmed increased resveratrol phenols loading in Res-PdNP-4 when compared to Res-PdNP-3; consequently Res-PdNP-4 nanoparticles were confirmed as the ideal nanoformulation to improve the bioavailability, biodistribution and emblematize as an adjuvant therapy to induce selective and specific tumor-cell-death.

The prostate tumor selective and specific affinity of Res-PdNP-4 nanoparticles through numerous cellular internalization studies undoubtedly revealed that Res-PdNP-4 nanoparticles can be internalized into prostate cancer cells via laminin receptor-mediated endocytosis which are receptors overexpressed on prostate cancer cells compared to normal cells. The Res-PdNP-4 nanoparticles were evaluated to investigate in vitro cellular toxicity against both prostate cancer (PC-3) cells and normal human aortic endothelial cells (HAEC). Results indicated that ResPdNP-4 exhibited comparable anticancer efficacy against prostate cancer cells as chemotherapeutic drugs (cisplatin and etoposide). However, the results showed that cisplatin and etoposide treatments were highly toxic to normal cells while Res-PdNP-4 nanoparticles presented no toxicity further corroborating laminin receptor-mediated delivery, making ResPdNP-4 nanoparticles selective and specific to prostate cancer cells.

Res-PdNP-4 nanoparticles were investigated in vivo using a human prostate tumor-bearing severely combined immunodeficient (SCID) male mice as the animal model to evaluate ResPdNP-4 nanoparticles ability to control or reduce prostate tumor size. The in vivo results of ResPdNP-4 showed a good dose response which was well tolerated by the animals, as no animal health problems and discomfort was observed as evidenced by body weight/eating habits of animals. Although further studies are required to determine a better dose to see increased 
efficacy. This study was performed through intravenous (IV) administration of the Res-PdNP-4, intraperitoneal (IP) delivery and direct injection into the tumor may show a better response as has been the case with many different types of nanoparticles. In conclusion, the therapeutic efficacy results showed that Res-PdNP-4 have significant therapeutic effect and are able to control the tumor size in comparison to the saline control and free resveratrol treated groups. This was due to the high corona of resveratrol-derived phenols and polyphenols on the PdNPs facilitating effectively enhanced delivery of resveratrol with high bioavailability, giving an advantage in tumor therapy. 


\section{CHAPTER 1}

\section{INTRODUCTION}

\subsection{Cancer}

Cancer continues to pose a tremendous challenge in society as being one of the main cause of death worldwide and contributes a major public health burden across the globe (Figure 1$){ }^{1-3}$ Cancer by definition is the uncontrollable cell proliferation that bypass the cell-cycle checkpoints; resulting in the creation of abnormal cells which become malignant tumors. ${ }^{4,5}$ The greatest number of cancer deaths is attributed by lung, breast, prostate, colorectal, liver, and stomach cancer (Figure 1) ${ }^{4-6}$ according to data reported by the International Agency for Research on Cancer with several entities such as the International Association of Cancer Registries, the National Center for Health Statistics (NCHS) - the National Cancer Institute (NCI), the Centers for Disease Control and Prevention's (CDC's), American Cancer Society, and the North American Association of Central Cancer Registries. In 2018, cancer was responsible for 9.6 million deaths worldwide and new cases are projected to rise above $70 \%$ in the next few decades. $^{7}$ Approximately 18.1 million new cases of cancer have been diagnosed globally in $2018 .^{7-9}$

In 2015, Weir et al. ${ }^{10}$ predicted an annual increase in cancer incidences in the United States among men to be $24.1 \%$ (>1 million cases) and 20.6\% among women (>900,000 cases) by 2020 . These increases are expected to be from breast, prostate, kidney, liver, lung, thyroid, urinary bladder uterus cancers. ${ }^{10}$ The United States alone accounts for an estimated 1,762,450 new cases diagnosed in 2019 that is equivalent to about 4,800 new cases each day. ${ }^{11}$ Advancements in cancer treatment in recent years have showed promise in alleviating cancer, however, the cost of 
cancer care is increasing at an exponential rate each year and this rise in health-care costs vastly represents a central challenge for governments and the private sectors. ${ }^{12-14}$ Moreover, late-stage cancer presentation and recondite diagnosis are common challenges that hinder the effectiveness of current treatment modalities due to the cancer's continuous evolution.

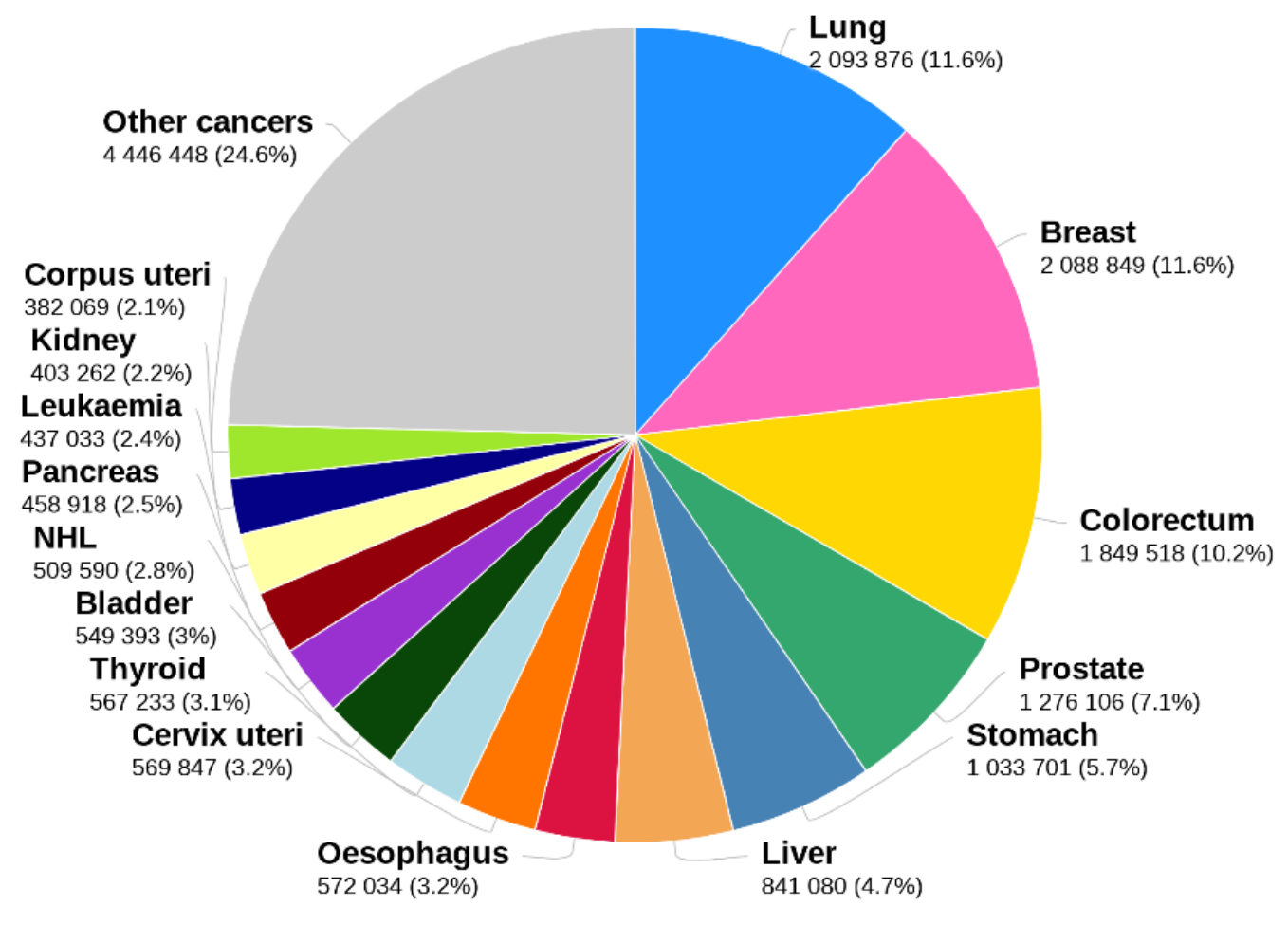

Total : 18078957

Figure 1: Cancer incidence worldwide in 2018. Breast, lung, prostate and colorectal cancers contribute a major public health burden across the globe. ${ }^{9}$

In 2017, Fleming et al. ${ }^{15}$ and Sayed et al. ${ }^{16}$ reported evidence suggesting that low-income countries (less than $30 \%$ ) lack pathology services that are accessible in the public sector. A majority $(\sim 90 \%)$ of high-income countries have appropriate cancer treatment services, ${ }^{4}$ therefore, the economic impact of cancer is significant causing financial burdens to families, countries and 
the world, with annual costs of cancer treatment that reached $\$ 1.16$ trillion in 2010 (Figure 2). ${ }^{17,18}$

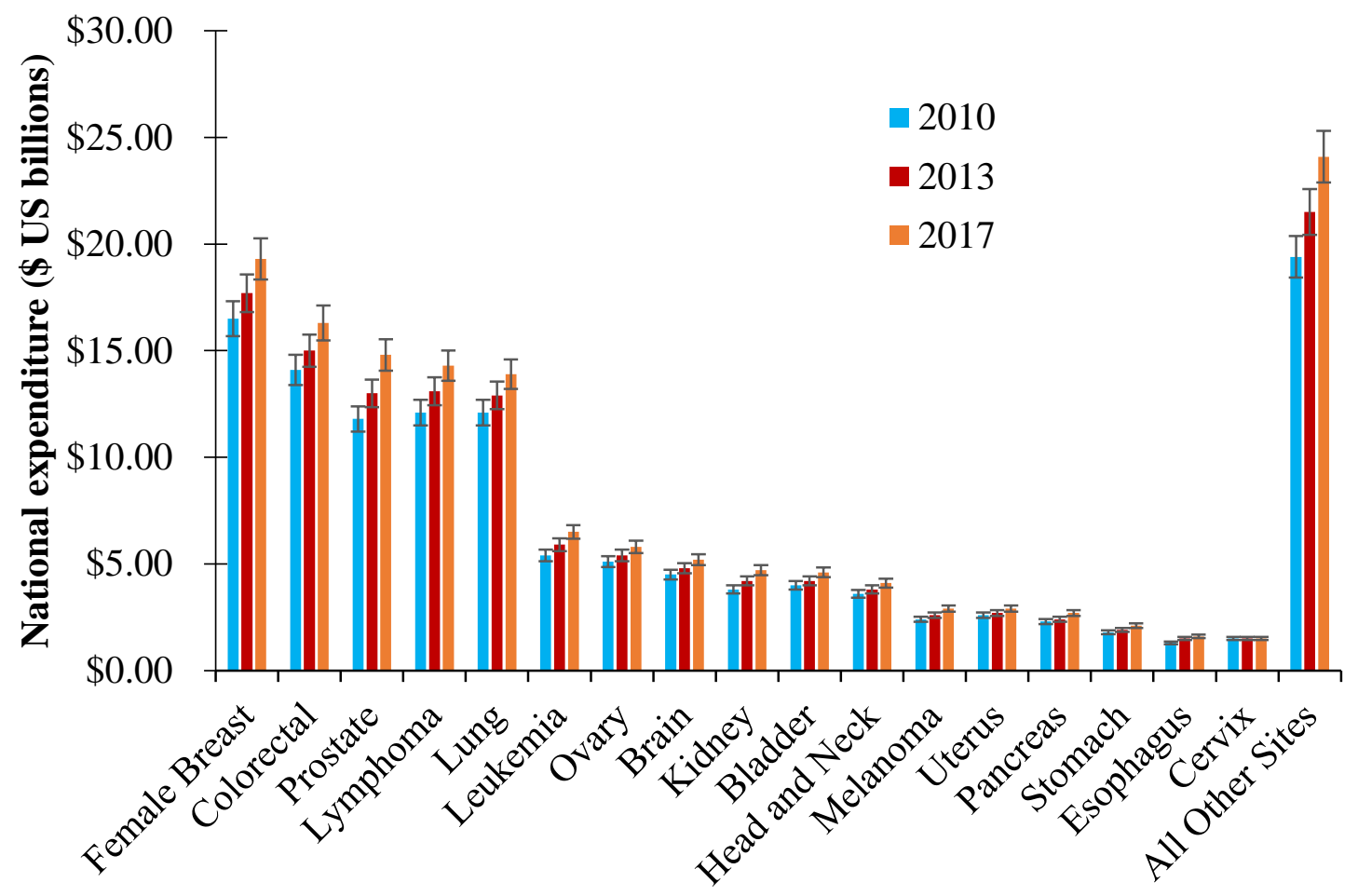

Cancer site

Figure 2: Financial burden of cancer healthcare in the United States from 2010 to $2017 .{ }^{17,18}$

The healthcare costs for cancer treatment are expected to increase exponentially as new and more advanced therapies are adopted as standard care for cancer treatments. Prostate cancer is an aggressive malignant tumour, reported to be the second-/sixth-leading cause of cancer-related death and the most diagnosed cancer in men in the United States and worldwide, respectively., ${ }^{9,19}$ The mainstay of cancer therapy modalities include chemotherapy, surgery, radiation therapy, targeted therapy, hormone therapy, stem cell transplant, cancer immunotherapy, and a combination of modalities. ${ }^{20}$ Some of these modalities have shown promising results towards prolonging the quality of life; however, failures in these therapies are governed by dose-limiting 
toxicities, lack of selectivity and specificity that leads to severe unintended side effects compromising the health and wellbeing of the patient.

In the case of cancer immunotherapy which has received tremendous attention to date due to reported benefits of activating the immune system to defend itself against tumour cells. ${ }^{21-25}$ This has sparked global interests in cancer treatment, where the global cancer immunotherapy market in 2016 was valued at $\$ 45$ billion. ${ }^{26}$ This market is projected to reach $\$ 152.83$ billion by 2024 , growing at a compound annual growth rate (CAGR) of 13.8\% during the forecast period of 2018 to $2024 .{ }^{27}$ The cancer cost conundrum for access to cutting-edge personalized cell-based therapies is an obstacle since the majority of the global population cannot afford this treatment due to price trajectory. ${ }^{28}$ Moreover, the Agency for Healthcare Research and Quality (AHRQ) estimated that the direct medical costs (total of all healthcare costs) for cancer in 2015 was $\$ 80.2$ billion. ${ }^{13}$ Despite modern medical approaches in cancer treatment, a major clinical problem and cause of patient death is attributed by secondary cancer events led by tumour metastasis.

\subsubsection{Prostate cancer}

Prostate cancer is the most common malignancy among men worldwide and the most frequently diagnosed type of cancer in men in over $57 \%$ of the countries in the world. ${ }^{9}$ Prostate cancer is associated with significant morbidity (1.3 million cases) and mortality $(359,000)$, representing the second most frequent cause of cancer-related deaths in male population in the world as shown in Figure 3., ${ }^{9,29}$ About $11 \%$ of men (aged 65 or older) will be diagnosed with prostate cancer in their lifetime, this number is expected rise according to the Global Cancer Observatory. ${ }^{9}$ In the United States alone an estimated number of 174,650 new cases of prostate cancer and 31,620 deaths from prostate cancer are expected for 2019., ${ }^{9,31,32}$ 


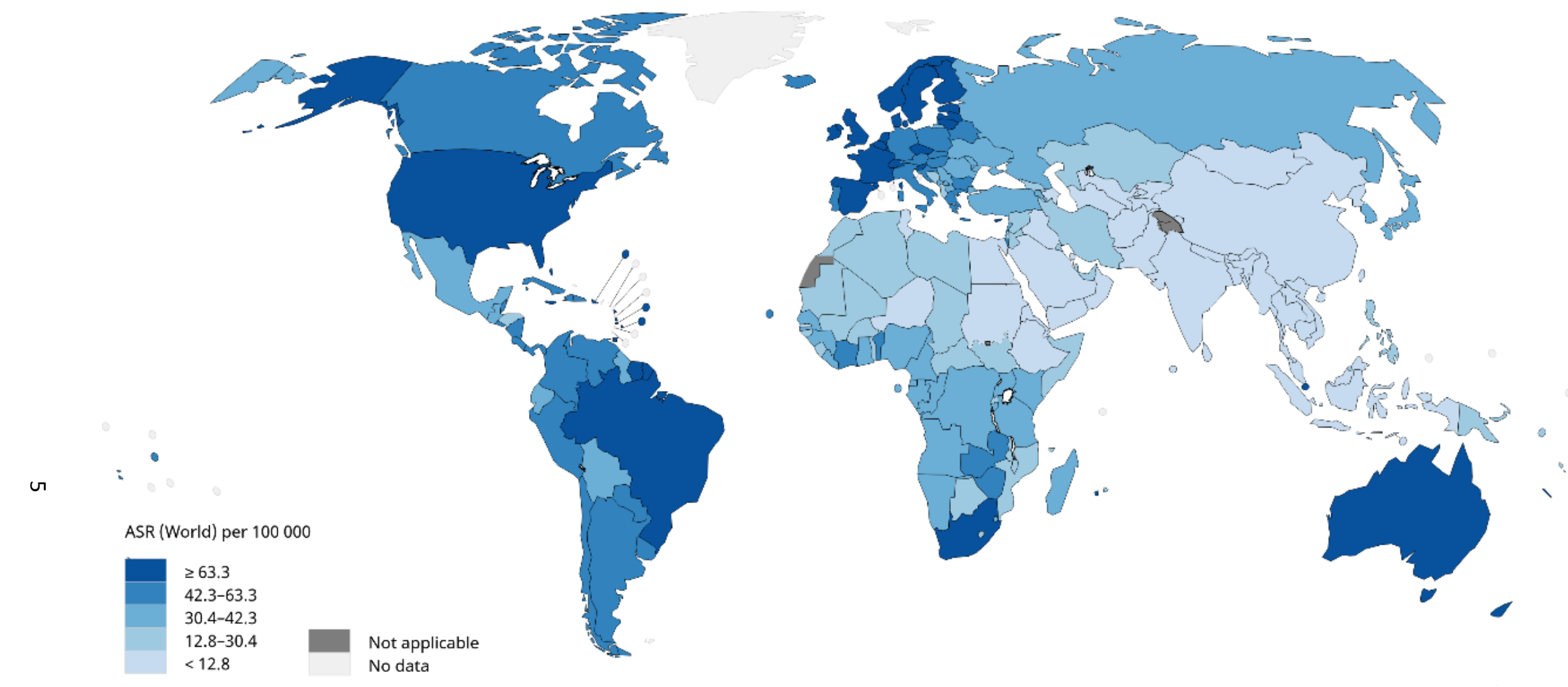

Figure 3: World map representing the most common type of cancer mortality in 2018 among men. Prostate cancer is ranked second after lung. Adapted with permission from GLOBOCAN 2018. 
Prostate cancer is a complex heterogeneous disease with diverse sets of hallmark capabilities that orchestrates prostatic tumorigenesis and metastasis (Figure 4).
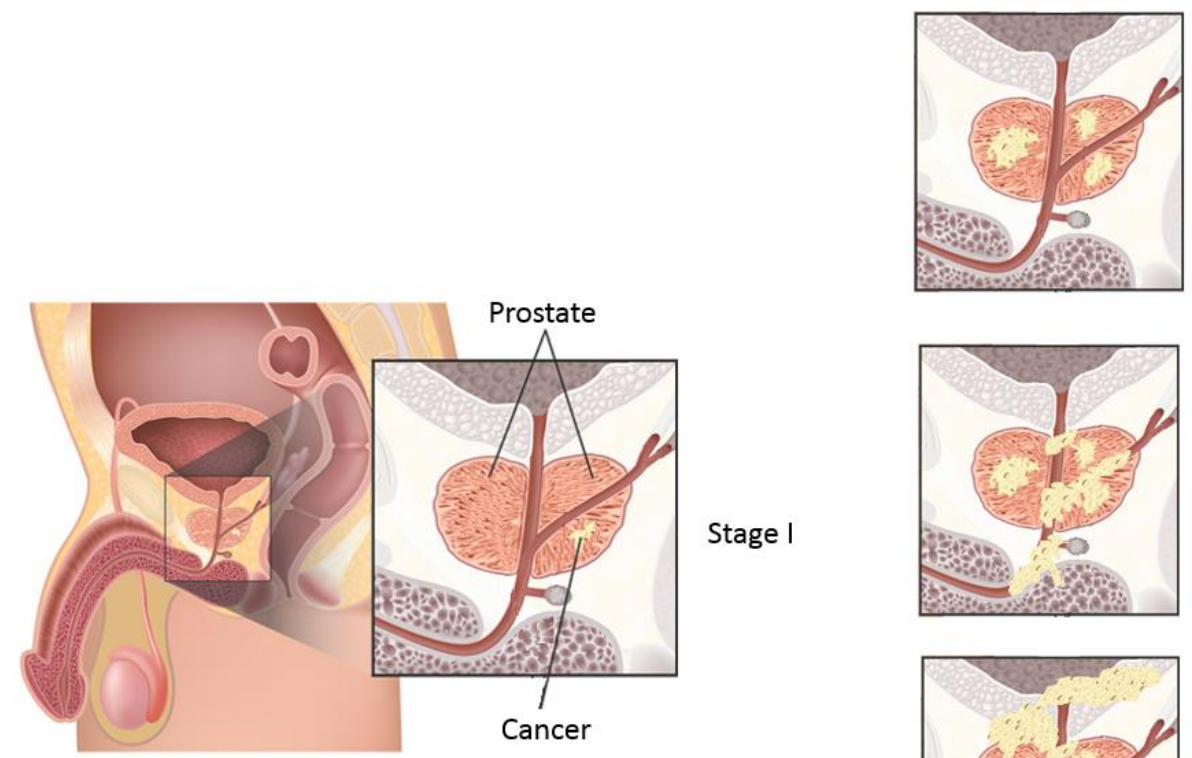

Stage II

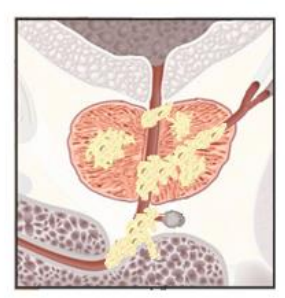

Stage III

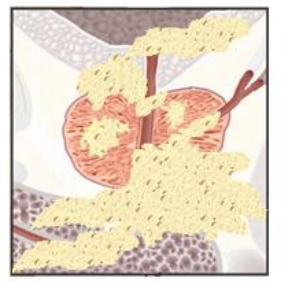

Stage IV

Figure 4: Prostate cancer tumorigenesis and metastasis from Stage I to Stage IV. The cancer cells grow within the prostate (Stage I and II), through the outer layer of the prostate into surrounding tissues (Stage III), and then to lymph nodes or other organs of the body (Stage IV). 


\subsubsection{Types of prostate cancer}

The type of prostate cancer is determined by the origin of the cancer:

\section{(i) Acinar and ductal adenocarcinoma}

The majority (95\%) of prostate cancers are adenocarcinomas, which are the frequent and most common type of diagnosed cancer in men. Acinar and ductal adenocarcinomas develop in the gland cells that line the prostate gland and in the cells that line the ducts (tubes) of the prostate gland, respectively. The ductal adenocarcinoma proliferates more rapidly than acinar adenocarcinoma. $^{33}$

\section{(ii) Transitional cell (or urothelial) cancer}

Approximately $4 \%$ of prostate cancers are arise from transitional cell carcinoma that develops from cells that line the inside of the tubes (ureters) which is part of the urinary tract, responsible for carry urine produced by the kidneys to the bladder and spreads into the prostate. ${ }^{34}$ It is rare solid malignant tumor that is highly aggressive and presents poor prognosis. ${ }^{35}$

\section{(iii) Squamous cell cancer}

This is a rare type of cancer develops from flat cells that cover the prostate and proliferates more rapidly than adenocarcinoma of the prostate. As demonstrated by Malik et al. ${ }^{36}$, Biswas et al. ${ }^{37}$ and Brunnhoelzl and $\mathrm{Wang}^{38}$, squamous prostate cancer approximately makes up $0.5 \%$ to $1 \%$ of all prostate carcinomas and aggressive cancer with bony metastases. 


\section{(iv) Small cell prostate cancer}

Rare type of anaplastic prostate cancer $(<1 \%)$ is a high-grade malignant neoplasm with neuroendocrine differentiation and one of the most aggressive malignancies of the prostate. ${ }^{39,40}$

\subsection{Diagnosis and current prostate cancer treatment}

The diagnosis of prostate cancer is mainly through monitoring prostate-specific antigen (PSA) or prostate-specific membrane antigen (PSMA) levels produced by normal and malignant cells of the prostate gland. ${ }^{41,42}$ The blood level of PSA/PSMA is elevated in men with prostate cancer, the PSA test is often in conjunction with a digital rectal exam, transrectal ultrasound, transrectal needle biopsy and these specify the appropriate type of treatment for a patient (Figure 5). ${ }^{42-45}$ The standard of treatment for prostate cancer depends type of diagnoses that range from localized to metastatic cancer as shown in Table 1 . However, many of the prostate tumors currently being diagnosed also include small, early stage, indolent, and low-risk prostate cancer. Substantial majority men still undergo overtreatment to receive aggressive whole-gland treatment with either surgery or radiation. 


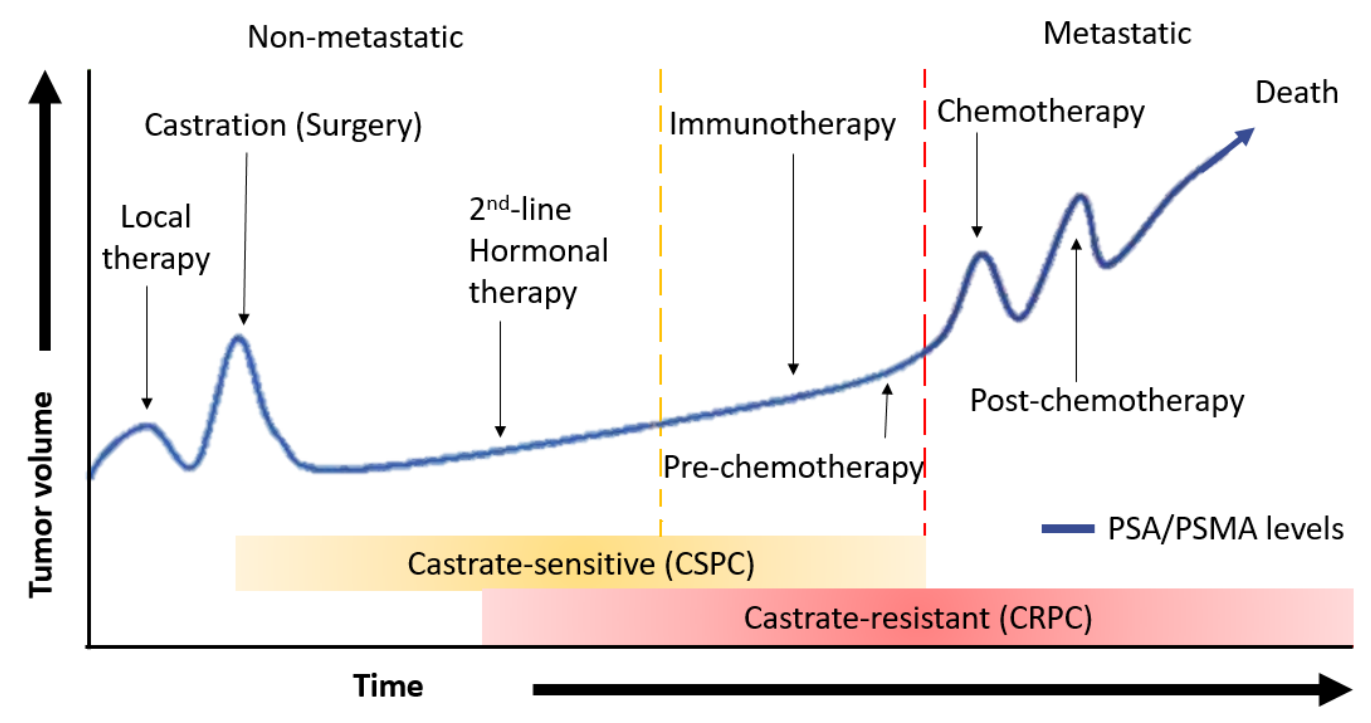

Figure 5: Systemic therapy landscape of prostate cancer progression. ${ }^{46,47}$ 
Table 1: Standard healthcare treatment for prostate cancer

\begin{tabular}{|c|c|c|}
\hline Treatment & Description & Side-effects \\
\hline $\begin{array}{l}\text { Expectant } \\
\text { management }\end{array}$ & $\begin{array}{l}\text { Expectant management consists of watchful waiting and active surveillance } \\
\text { of prostate cancer progression that includes treating symptoms with palliative } \\
\text { intent focused on managing pain or urinary difficulty (e.g. urinary retention } \\
\text { or hematuria) - candidates are typically older (>65 years). }{ }^{48} \text { Active } \\
\text { surveillance involves a series of monitoring prostate-specific antigen (PSA) } \\
\text { levels and a combination of prostate biopsies and physical examinations to } \\
\text { monitor cancer progression with curative intent. }{ }^{43}\end{array}$ & $\begin{array}{l}\text { May be more detrimental effects } \\
\text { for younger patients with high } \\
\text { risks of progression. }\end{array}$ \\
\hline Surgery & $\begin{array}{l}\text { Surgical removal of the tumor using three types of acceptable surgeries (i) } \\
\text { radical prostatectomy (retropubic or perineal) by removing the prostate } \\
\text { through an incision in the abdominal wall or in the perineum, respectively; } \\
\text { (ii) pelvic lymphadenectomy (PLDN) - removal of the lymph nodes in the } \\
\text { pelvis and (iii) transurethral resection of the prostate to remove tissue from } \\
\text { the prostate using a resectoscope inserted through the urethra. }{ }^{49-51}\end{array}$ & $\begin{array}{l}\text { Erectile dysfunction, damage to } \\
\text { nearby organs, and infections at } \\
\text { the surgery site. }\end{array}$ \\
\hline
\end{tabular}




\begin{tabular}{|c|c|c|}
\hline Treatment & Description & Side-effects \\
\hline $\begin{array}{l}\text { Radiation } \\
\text { therapy and } \\
\text { radiopharmaceu } \\
\text { tical therapy }\end{array}$ & $\begin{array}{l}\text { Radiotherapies are the second major therapeutic modalities for prostate } \\
\text { cancer. These utilizes a form of high-energy } \mathrm{x} \text {-rays or other types of } \\
\text { radiation ( } \alpha \text { - or } \beta \text { particles) to kill the cancer cells. The treatment strategies } \\
\text { include external-beam radiotherapy (EBRT), hypofractionated radiation } \\
\text { therapy, internal radiation therapy/brachytherapy - this involves inserting } \\
\text { radioactive seeds }\left(\mathrm{t}_{1 / 2}=60 \text { days) at the prostate tumor site and monitoring }\right. \\
\text { tumor shrinkage via ultrasound guidance. Radiopharmaceutical therapy uses } \\
\text { radioactive (e.g. radium-223) to treat prostate cancer that has metastasized to } \\
\text { the bone. }\end{array}$ & $\begin{array}{l}\text { Increased risk of having bladder } \\
\text { and/or gastrointestinal cancer. } \\
\text { Damage to nearby organs and } \\
\text { infertility. }\end{array}$ \\
\hline $\begin{array}{l}\text { Hormonal } \\
\text { therapy }\end{array}$ & $\begin{array}{l}\text { Surgical removal of male hormones or silencing their expression through } \\
\text { orchiectomy, androgen deprivation therapy (antiandrogens), abiraterone } \\
\text { acetate, estrogens, luteinizing hormone-releasing hormone agonists and other } \\
\text { drugs that prevent adrenal glands. }\end{array}$ & $\begin{array}{l}\text { Impaired sexual function, loss of } \\
\text { desire for sex, and weakened } \\
\text { bones. }\end{array}$ \\
\hline Chemotherapy & $\begin{array}{l}\text { Treatment with drugs such as taxane (docetaxel and cabazitaxel) and } \\
\text { platinum-based (cisplatin, carboplatin and oxaliplatin) chemotherapies that } \\
\text { stop the proliferation of cancer cells. }{ }^{54,55}\end{array}$ & $\begin{array}{l}\text { Secondary systemic toxicity, hair } \\
\text { loss and diarrhea. }\end{array}$ \\
\hline
\end{tabular}




\begin{tabular}{|c|l|l|}
\hline Treatment & \multicolumn{1}{c|}{ Description } & \multicolumn{1}{c|}{ Side-effects } \\
\hline Immunotherapy & $\begin{array}{l}\text { Boosting or restoring the immune homeostasis against cancer. Sipuleucel-T } \\
\text { and pembrolizumab used for programming a patient's immune system to } \\
\text { target prostate cancer cells and destroy them. }\end{array}$ & $\begin{array}{l}\text { Bones,57 } \\
\text { resolved within a few days. The } \\
\text { biggest challenge is the cost of } \\
\text { the treatment. }\end{array}$ \\
\hline
\end{tabular}


The challenge of current prostate cancer therapies is hindered by the pivotal factors that constitute an 'Achilles heel' where the systemic side effects (such as urinary symptoms and sexual dysfunction) greatly outweigh the benefits of the treatment. There is an urgent need to develop multimodality approaches that simultaneously targets cancers at multiple levels.

\subsection{Significance}

Metal-based therapies such as platinum (Pt)-based antitumor chemotherapeutic drugs (cisplatin [cis- $\mathrm{Pt}\left(\mathrm{NH}_{3}\right) \mathrm{Cl}_{2}$ ], carboplatin and oxaliplatin have been used as broad spectrum therapeutic agents to primarily treat solid tumors and these chemotherapeutic agents remain among the most widely used cancer therapies for myriad human cancers (Figure 6) ${ }^{58}$ The quest for metal-based anticancer agents continues to attract significant attention because of the evidence that metals such as Pt show propensity to form DNA intercalating adducts which are generally not feasible through conventional drugs. ${ }^{59,60}$ Although cisplatin and its derivatives (carboplatin and oxaliplatin) are used in the vast majority cancer treatments for patients suffering from various forms of cancer, these Pt-based pharmaceuticals continue to pose vexing clinical problems such as gastrointestinal and cardiovascular toxicity, injury to renal tubular epithelial cells, emetogenesis, nephrotoxicity and others. ${ }^{58,61,62}$ Pt-based drugs are highly toxic with a lethal dose (LD) of $6.6 \mathrm{mg} / \mathrm{Kg}$ of body weight for cisplatin and the low tumor selectivity, specificity and solubility also contribute to its high toxicity. ${ }^{63}$ Moreover, cisplatin-resistant tumors cannot be treated through Pt-based chemotherapeutic agents. ${ }^{64}$

To circumvent the challenges presented by Pt-based chemotherapeutic drugs, researchers have devoted significant amount of work in developing non-Pt metal ion complexes for cancer treatment. Palladium (Pd) complexes as anti-cancer drugs have been explored due to their 
structural similarities and overlapping coordination chemistry with Pt as shown in Table $2 .^{58,65}$ Pd-based pharmaceuticals are being developed for use as the next generation metal-based anticancer drugs in order to overcome severe systemic toxic effects and drug resistance associated with cisplatin and its derivative chemotherapeutic agents. ${ }^{58,66}$

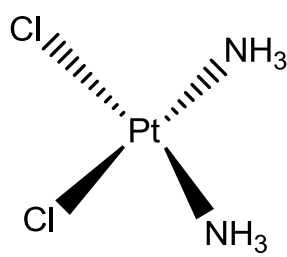

(i)<smiles>N[P+]1(N)OC(=O)C2(CCC2)C(=O)O1</smiles>

(ii)<smiles>O=C1O[P]2(NC3CCCC[C@@H]3N2)OC1=O</smiles>

(iii)

Figure 6: Platinum chemotherapeutic drugs used for the treatment of solid cancer (i) cisplatin, (ii) carboplatin and (iii) oxaliplatin

Table 2: Properties of Pd(II) and Pt(II)

\begin{tabular}{|l|l|l|}
\hline \multicolumn{1}{|c|}{ Property } & \multicolumn{1}{|c|}{ Pd(II) } & \multicolumn{1}{|c|}{ Pt(II) } \\
\hline Dissociation pattern & Slow & Fast \\
\hline Density $\left(\mathrm{g} \mathrm{cm}^{-3}\right)$ & 12.0 & 21.5 \\
\hline Reactivity & High & Low \\
\hline
\end{tabular}

Albeit, $\mathrm{Pd}(\mathrm{II})$ and $\mathrm{Pt}(\mathrm{II})$ have significant coordination chemistry similarities, $\mathrm{Pd}(\mathrm{II})$ complexes serve as suitable antitumor drugs over Pt(II) complexes mainly because of their high solubility. ${ }^{67}$ 


\subsection{Palladium complexes used for cancer therapy}

Palladium is known to possess remarkable catalytic and optical properties, however; its medical applications have not been fully exploited. ${ }^{68}$ It is widely used as a versatile catalyst in a number of important reactions (e.g. C-C coupling reactions, hydrogen storage and sensing) ${ }^{69}$ Moreover, Pd exhibits exceptional electro-activity that can be harnessed for high-energy flux production. ${ }^{70}$ However, Pd complexes have been used in the medical sector for several years. For example, palladium chloride complexes was previously prescribed for the treatment of tuberculosis, administered at $1 \mathrm{mg} / \mathrm{kg}$ body weight per day without presenting side effects. ${ }^{71}$ In the medical field, Pd has been used as an alloy component in dental appliances and radioactive isotope of $\mathrm{Pd}$ $\left({ }^{103} \mathrm{Pd}\right.$ seeds) are used for brachytherapy for melanoma and prostate cancer treatments. ${ }^{72,73}$

Jahromi et al. ${ }^{58}$ reported Pd(II) dithiocarbamate derivatives complexes were effective against cisplatin resistant tumors. Noticeably, the chelating ligand was found to play a crucial role in the effectiveness of these complexes, since some Pd(II) complexes shown no significant antitumor efficacy. Furthermore, Jahromi et al. ${ }^{58}$ demonstrated that these complexes exhibited a low kidney toxicity compared to cisplatin, this was associated with the inability of kidney tubules proteins to replace tightly bound chelate ligands of $\mathrm{Pd}(\mathrm{II})$. 


\subsubsection{Types of Pd(II) complexes}

A variety of $\mathrm{Pd}(\mathrm{II})$ complexes have been investigated for their in vitro and in vivo anticancer activity against a number of tumors (Figure 7).

(i) Trans-Pd(II) complexes - Abu-Surrah et al. ${ }^{65}$ reported that trans-Pd(II) complex (trans- $\left[\operatorname{Pd}\left\{(\mathrm{R})-(+)\right.\right.$-bornylamine $\left.\left.{ }_{2} \mathrm{Cl}_{2}\right]\right)$, when compared with cisplatin, showed similar antitumor activity against HeLa cells. Huq et al. ${ }^{74}$ found that trans- $\mathrm{PdCl}_{2} \mathrm{~L}_{2}$ where $\mathrm{L}=$ 2-hydroxypyridine, had a higher antitumor activity against resistant ovarian cancer cell lines.

(ii) Pd(II) complexes bidentate ligands - square planar $\mathrm{Pd}(\mathrm{II})$-selenite complex ([(bipy)Pd( $\left.\left.\mathrm{SeO}_{3}\right)\right]$ ) was found to intercalate DNA through covalent bonding similar to cisplatin mode of action, results showed better cytotoxicity when compared to cisplatin. ${ }^{65}$ The N-N ligands (bipyridine and phenanthroline) based Pd(II) complexes have been reported to have lower half maximal inhibitory concentration $\left(\mathrm{IC}_{50}\right)$ values than cisplatin against P388 lymphocytic leukemia cells. ${ }^{75}$

(iii) Phosphine ligands coordinated Pd(II) complexes - The cyclopalladated complexes based on biphosphine ligands were reported to cause total destruction of the tumor cells (B16F10-Nex2 murine melanoma; Ehrlich ascites carcinoma and DU145 human prostate carcinoma $(\mathrm{HTB}-81))$ at low concentration $(<1.25 \mu \mathrm{M}) .^{76}$

(iv) Pd(II) complexes with mixed donor atom ligands $[($ Benzyl $) \operatorname{Pd}\{$ bis(thiosemicarbazonate $)\}]$ is a thiosemicarbazide derivative that was found to have similar $\mathrm{IC}_{50}$ of cisplatin and still active against cisplatin-resistant cell lines. $^{77}$ 
(v) Multinuclear Pd(II) complexes - a trinuclear complex $\left[\left\{\right.\right.$ trans- $\left.\mathrm{PtCl}\left(\mathrm{NH}_{3}\right)\right\} 2-\#-$ \{trans- $\mathrm{Pd}\left(\mathrm{NH}_{3}\right)$ (2-hydroxypyridine)- $\left.\left(\mathrm{H}_{2} \mathrm{~N}\left(\mathrm{CH}_{2}\right)_{6} \mathrm{NH}_{2}\right)_{2}\right]^{4+}$ was reported to have antitumor activity against cancer cell lines. ${ }^{78}$ This was found to be attributed to the formation of inter-strand GG adducts with duplex DNA which affects the integrity of the DNA leading to apoptosis.

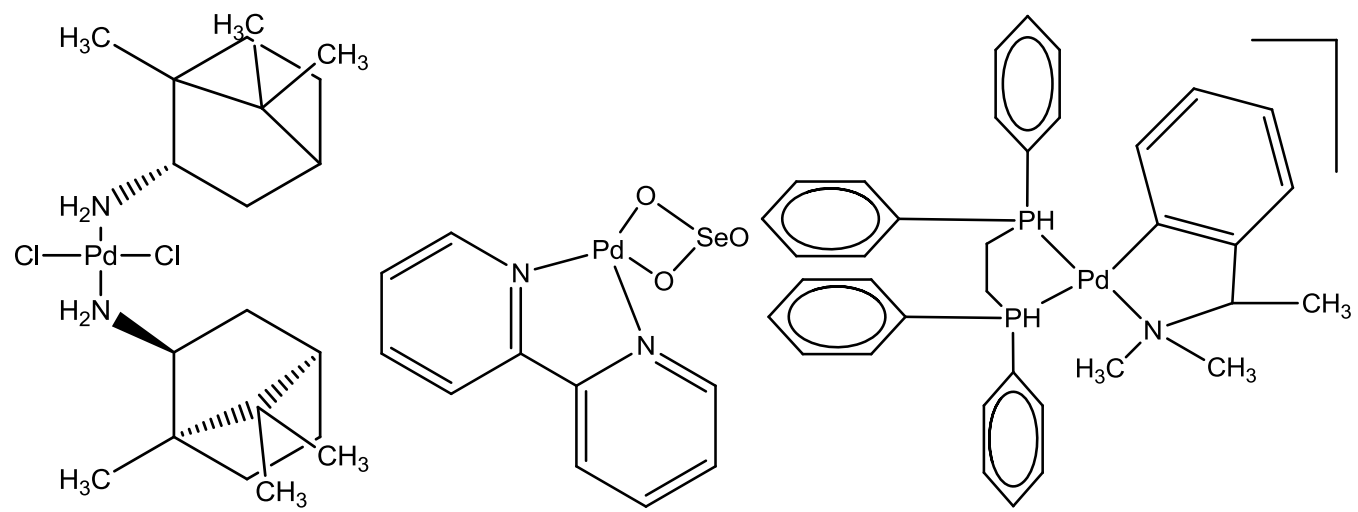

(i)<smiles></smiles>

(iv)

(ii)<smiles>CCCCCCCCN[PH](N)(NCC)[n+]1ccccc1O</smiles><smiles>CCCCCCCCN[PH](N)(NCC)[n+]1ccccc1O</smiles>

(iii)

(v)

Figure 7: Palladium complexes that have been investigated for their activity against tumor cells (i) trans-[Pd$\{(\mathbf{R})-(+)-\text { bornylamine }\}_{2} \mathrm{Cl}_{2}$, (ii) $\left[(\right.$ bipy $\left.) \operatorname{Pd}\left(\mathrm{SeO}_{3}\right)\right]$, (iii) $\operatorname{Pd}(\mathrm{II})$ complexes bearing 1,2-bis(diphenylphosphino)ethane (dppe), (iv) [(Benzyl)Pd\{bis(thiosemicarbazona te)\}], and (v) [\{trans-PtCl( $\left.\left.\mathrm{NH}_{3}\right)\right\} 2-\#-\left\{\right.$ trans-Pd( $\left.\left.\mathrm{NH}_{3}\right)\left(2 \text {-hydroxypyridine)-( } \mathrm{H}_{2} \mathbf{N}\left(\mathrm{CH}_{2}\right)_{6} \mathrm{NH}_{2}\right)_{2}\right]$ 4+ 65 


\subsubsection{Challenges associated with Pd(II) complexes}

The pharmaceutical design of $\mathrm{Pd}(\mathrm{II})$ complexes with anticancer activity are challenging, since $\mathrm{Pd}(\mathrm{II})$ is thermodynamically and kinetically less stable than $\mathrm{Pt}(\mathrm{II})$ complexes. ${ }^{79}$ Moreover, $\mathrm{Pd}(\mathrm{II})$ complexes have rapid ligand-exchange kinetics $\left(10^{5}\right.$ times faster than their corresponding $\mathrm{Pt}$ analogues) $^{65}$ leading to very reactive species that hinders it's overall pharmacological effectiveness. ${ }^{80}$ Therefore, it is important to take into consideration the high reactive nature of Pd(II) when developing Pd(II)-based pharmaceutical drugs, Abu-Surrah et al. ${ }^{65}$ suggested that Pd(II) antitumor drugs should be stabilized by strongly coordinated nitrogen ligand and suitable non labile leaving group to maintain structural integrity in vivo. However, rapid ligand exchange and hydrolysis is a major drawback of palladium complexes that limits their clinical translation. This can be overcome by the palladium nanostructures, to be discussed below. ${ }^{79}$ Isomerization of Pd(II) complexes from trans to cis conformation affects their bioactivity. ${ }^{81,82}$ In general, several studies indicated that trans-Pd(II) complexes are more effective than cis-Pd(II) complexes and show activities that are comparable or superior to those of cisplatin against tumor cells. ${ }^{65}$

\subsection{Plant-derived agents as anticancer pharmaceutical drugs for cancer therapy}

Biologically active compounds isolated from plants are considered to be nutraceuticals because they possess some medicinal properties which can lead to the treatment of a variety of certain diseases as shown in Scheme 1. The use of medicinal plants for treating diseases dates back to Egyptians, Greek and Romans civilizations, however, 3000 years ago; Hippocrates coined the phrase "Let food be thy medicine and medicine be thy food" - a notion about the therapeutic application of food. ${ }^{83}$ This is justified by modern medicine, such as plant derived chemotherapies 
used for the treatment of various disorders such as cancer. There are four classes of plant-derived anticancer and other disease-related drugs currently in the market as shown in Table $3 .^{84}$

Modern medicine is focused on the fundamental premise of understanding the pharmacokinetics and pharmacodynamics in relation to the dosimetry of a drug rather than complex formulations by medicinal practitioners (e.g. herbalist and traditional healers) that lack reproducibility between batches. All plant-derived anticancer drugs on the market are based on reverse engineering and pharmacology as shown in Scheme 1 to isolate the single most active compound and determining their molecular mechanism and mode of action. ${ }^{85}$ This is dependent on the quality, purity and quantity of the isolated active compound from the medicinal plant and further characterization to determine the pharmacokinetics, pharmacodynamics, metabolic fate, efficacy and safety, dosimetry, drug interaction, immunogenicity and associated side effects.

However, most drugs that reach the market are based on the positive statistical correlations, safety and efficacy on a large population. As we encroach more into approaches geared at personalized medicine and recent discoveries in immunotherapy - the future of modern medicine is immunomodulation and regenerative medicine to strengthen the immune system. Medicinal plants serve as reservoirs for novel bioactive chemical entities as potential candidates for the development of anticancer drugs due to their pleiotropic actions specifically against tumor cells without affecting normal cells. ${ }^{86}$ Several nutraceuticals have been reported for playing a pivotal role by inhibiting the progression and development of cancer trough convulsing cancer cell activating proteins/enzymes and signalling pathways. 


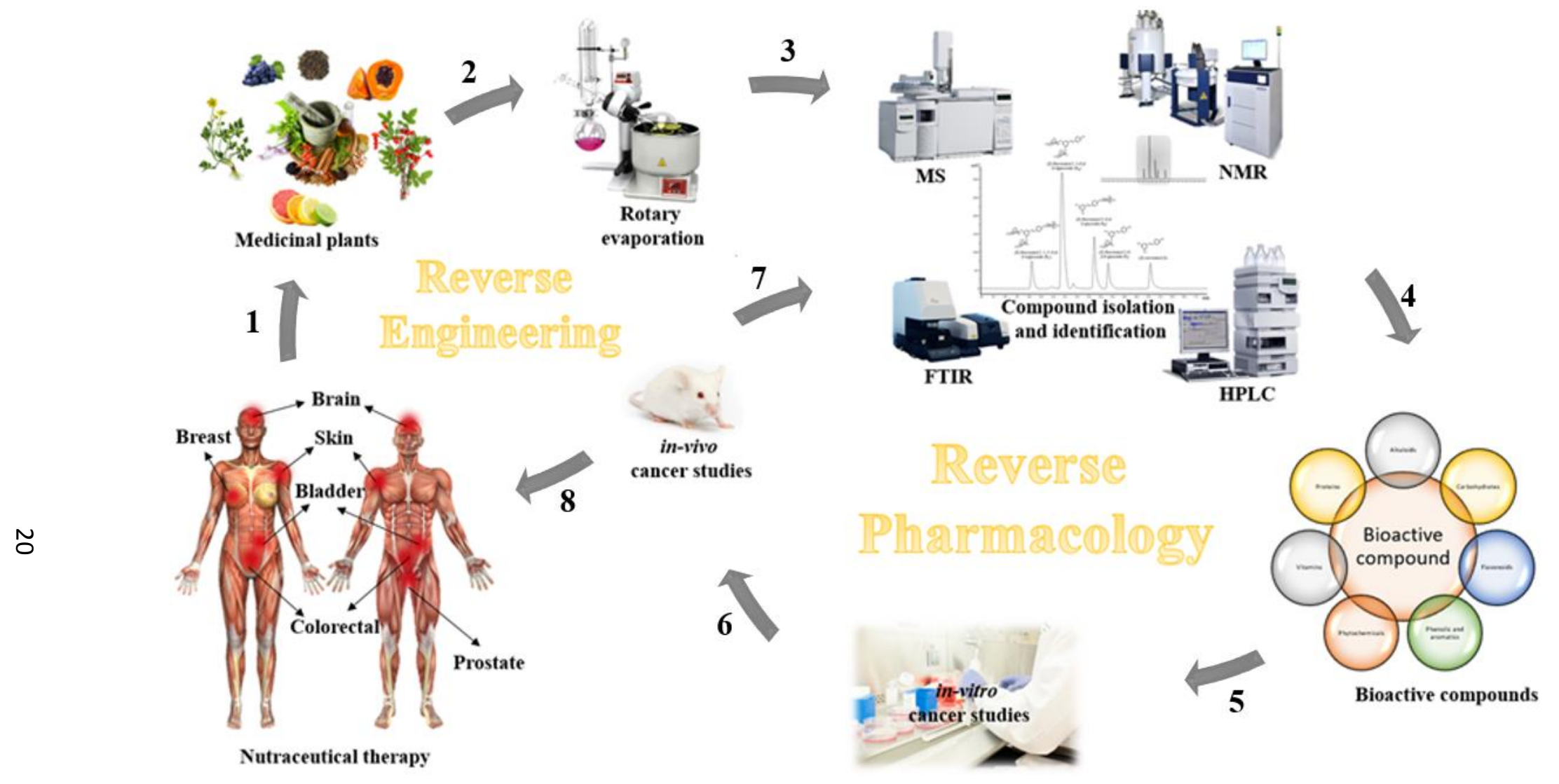

Scheme 1: Reverse engineering and pharmacology of plant-derived anticancer drugs 1) food as a source of nutrients, 2) chemical extraction from plant material, 3) purification and identification of isolated compounds, 4) identification of active compound, 5) in-vitro cellular studies, 6) in-vivo anticancer pre-clinical studies, 7) bioinformatics and immunological studies and 8) clinical studies for prospective use as a cancer therapeutic drug. 
Table 3: Plant-derived drugs in the market used for the treatment of cancer and other-related diseases

\begin{tabular}{|c|c|c|c|c|c|c|}
\hline Class & $\begin{array}{c}\text { Anticancer } \\
\text { drugs }\end{array}$ & Structure & Source & $\begin{array}{c}\text { Therapeutic } \\
\text { benefits }\end{array}$ & Company & Reference \\
\hline Taxanes & $\begin{array}{l}\text { Paclitaxel } \\
(\text { Taxol } \AA) \text { and } \\
\text { Docetaxel } \\
\text { (Taxotere } \AA)\end{array}$ & (Paclitaxel) & $\begin{array}{l}\text { Plant } \\
\text { alkaloid } \\
\text { derived from } \\
\text { the Pacific } \\
\text { yew (Taxus } \\
\text { brevifolia). } \\
\text { Docetaxel is } \\
\text { the synthetic } \\
\text { derivative of } \\
\text { paclitaxel, } \\
\text { 10-deacetyl } \\
\text { baccatin III } \\
\text { as the } \\
\text { precursor. }\end{array}$ & $\begin{array}{l}\text { Mitotic inhibitor } \\
\text { which binds } \\
\text { microtubulin } \\
\text { preventing } \\
\text { chromosomal } \\
\text { missegration, } \\
\text { apoptotic cell death } \\
\text { and mitotic arrest. } \\
\text { It is used as a cancer } \\
\text { chemotherapy for } \\
\text { breast, lung, } \\
\text { pancreatic, prostate, } \\
\text { melanoma and } \\
\text { ovarian cancer. }\end{array}$ & $\begin{array}{l}\text { Python Biotech, } \\
\text { LLC } \\
\text { (United States) }\end{array}$ & $\begin{array}{l}\text { Weaver }^{87} ; \\
\text { Pandey et al. }\end{array}$ \\
\hline
\end{tabular}




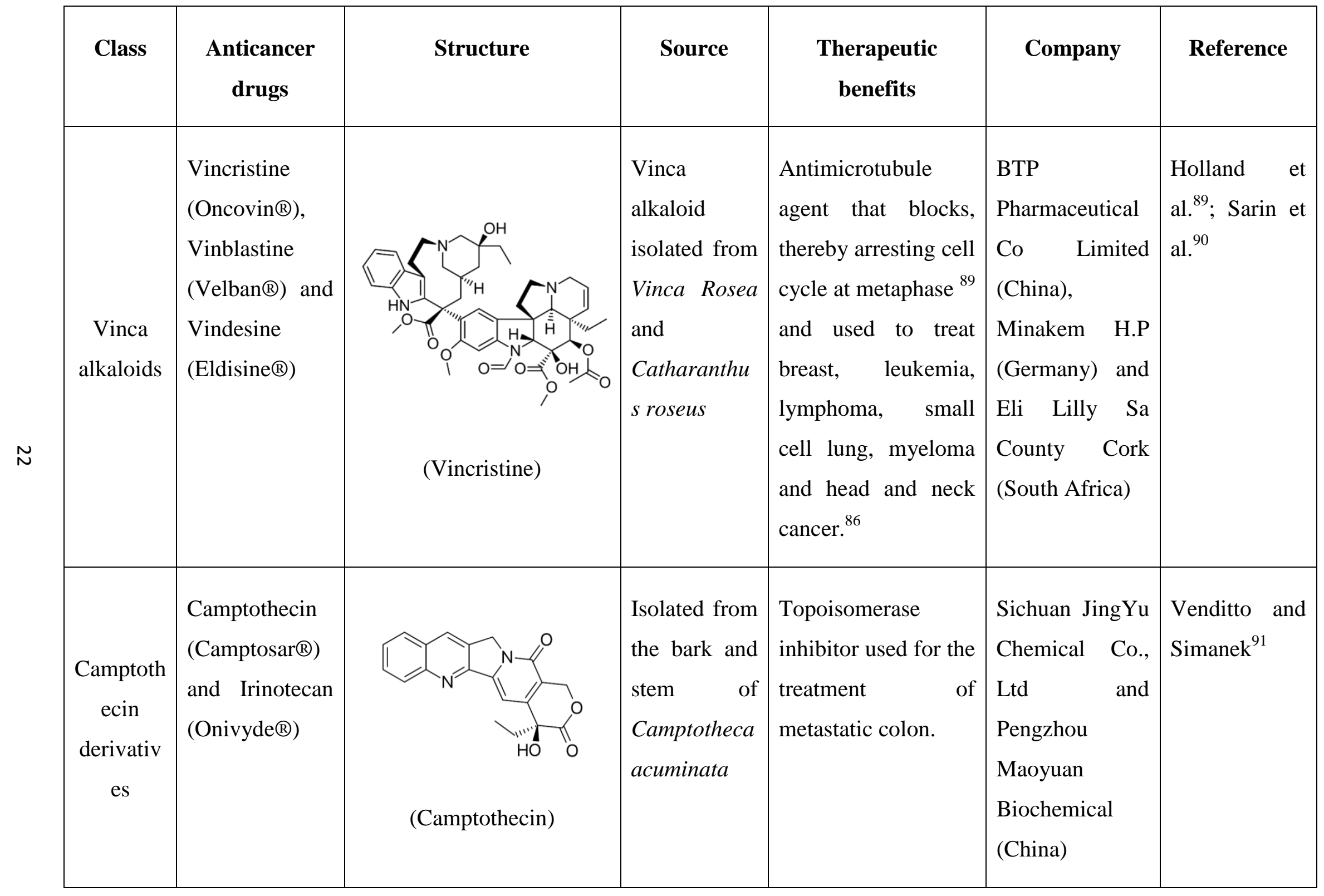




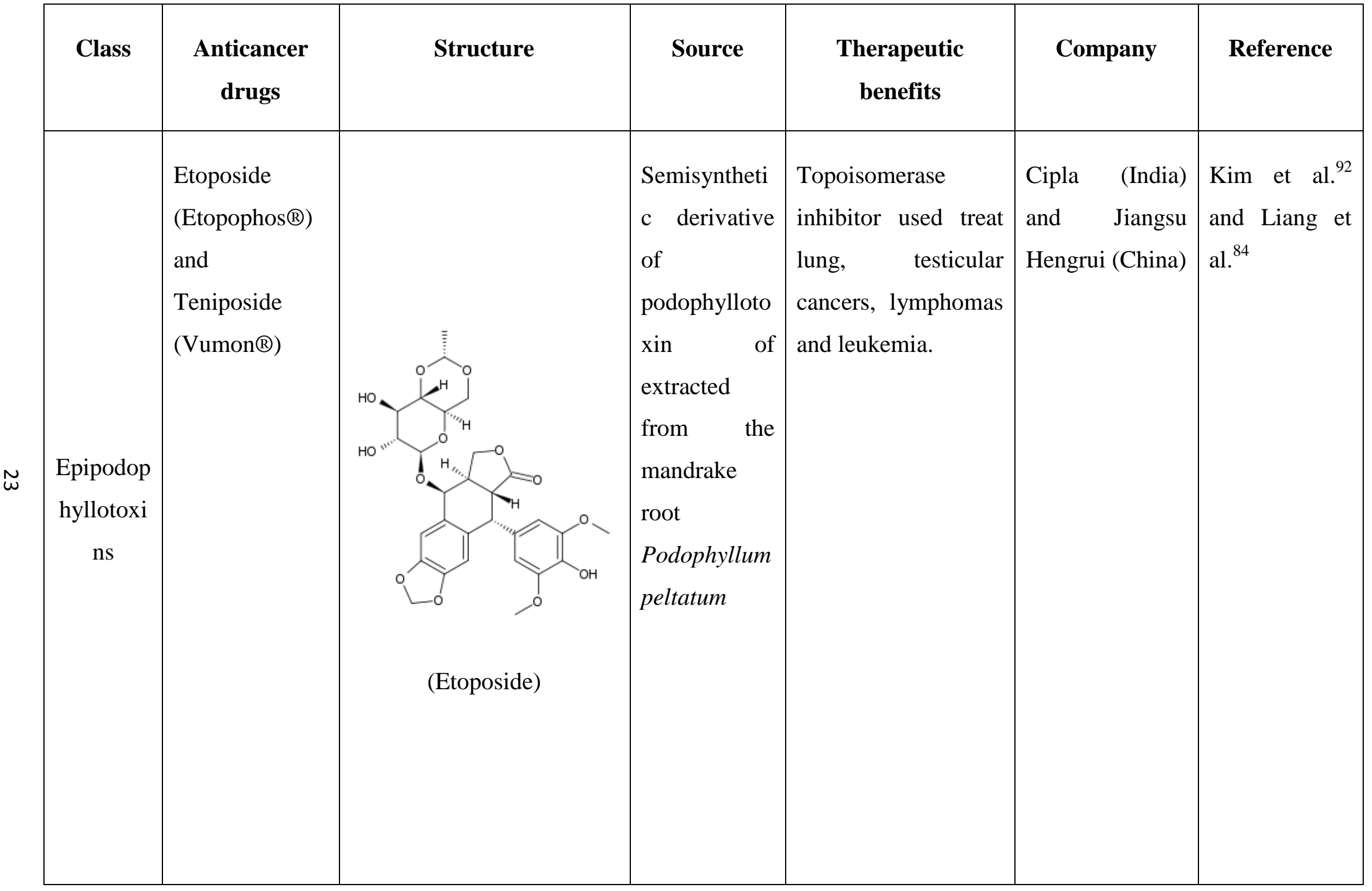




\begin{tabular}{|c|c|c|c|c|c|c|}
\hline \multicolumn{7}{|c|}{ Other disease related drugs } \\
\hline Class & $\begin{array}{l}\text { Anticancer } \\
\text { drugs }\end{array}$ & Structure & Source & $\begin{array}{l}\text { Therapeutic } \\
\text { benefits }\end{array}$ & Company & Reference \\
\hline $\begin{array}{l}\text { Cephalot } \\
\text { axus } \\
\text { alkaloids }\end{array}$ & $\begin{array}{c}\text { Omacetaxine } \\
\text { mepesuccinate } \\
(\text { Synribo®) }\end{array}$ & & $\begin{array}{l}\text { Isolated from } \\
\text { Cephalotaxu } \\
s \\
\text { harringtonii }\end{array}$ & $\begin{array}{l}\text { Tyrosine kinase } \\
\text { inhibitor used for the } \\
\text { treatment of chronic } \\
\text { myeloid leukemia. }\end{array}$ & $\begin{array}{l}\text { Teva } \\
\text { Pharmaceutical } \\
\text { (United States) }\end{array}$ & $\begin{array}{l}\text { Alvandi et } \\
\text { al. }{ }^{91}\end{array}$ \\
\hline \multicolumn{2}{|c|}{ Anticancer drugs } & Structure & Source & $\begin{array}{l}\text { Therapeutic } \\
\text { benefits }\end{array}$ & Company & Reference \\
\hline \multicolumn{2}{|c|}{ Epidiolex } & & $\begin{array}{l}\text { Cannabidiol } \\
\text { drug from } \\
\text { the cannabis } \\
\text { plant }\end{array}$ & $\begin{array}{l}\text { to treat seizure } \\
\text { disorders }\end{array}$ & $\begin{array}{l}\text { GW } \\
\text { Pharmaceuticals }\end{array}$ & $\mathrm{FDA}^{93}$ \\
\hline
\end{tabular}




\subsection{Resveratrol}

Resveratrol (3,5,4'-trihydroxy-trans-stilbene) is a non-flavonoid polyphenolic compound predominantly found in the skin of red grapes and various red wines. ${ }^{94}$ Resveratrol (Res) was investigated because of the "French paradox" - that is, the low prevalence of coronary heart disease were reported in a population of people in Southern France as a result of high red wine consumption. ${ }^{95-97}$ This posed extensive research on Res, thus several studies have been focused on demonstrating the cardioprotective properties of Res against plaque development in various animal models of atherogenesis. ${ }^{98-100}$ Nonetheless, like most effective polyphenols, low bioavailability hinders its effectiveness towards clinical translation. A minimal fraction (approximately $\sim 2-4 \mu \mathrm{M}$ in the blood) of ingested Res reaches mammalian tissue and to some extent the tumor. ${ }^{101}$ Therefore, the increased delivery of Res to the tumor is paramount to improving its bioavailability for an increased therapeutic effect against cancer and other related diseases. Coimbra et al. ${ }^{102}$ encapsulated Res in liposomes to increase its bioavailability and reduce their enzymatic degradation. ${ }^{94}$

A pharmacokinetic study of Res was carried out by Ortuno et al. ${ }^{103}$ demonstrated that Res from natural grape products had a higher absorption in vivo as opposed to tablet supplements, thereby conferring the importance of the delivery matrix for Res which imposes its bioavailability. ${ }^{104}$ Moreover, Res is a lipophilic molecule that can easily cross through membranes and target membrane receptors (such as estrogen receptor alpha and beta (ER- $\alpha$ and ER- $\beta$ ), integrin $\alpha v \beta 3$, androgen receptor) that can increase its intra-tissular levels. ${ }^{105-109}$ According to some studies, Res is known to be a chemopreventive molecule with various health benefits, such as antiangiogenic, anti-diabetic, anti-obesity, anti-inflammatory, anti-oxidant, anti-proliferative, anti- 
viral, cardiovascular, anti-cancer and neuroprotective properties (Figure 8). ${ }^{83,110-112}$ As a natural anticancer agent, Res participates in the regulation of a number of signaling pathways resulting in suppression of adhesion, thus limiting invasion and metastasis of tumor cells. ${ }^{109}$ Furthermore, tumor cell proliferation is affected due to induction of apoptosis, differentiation and reduced angiogenesis facilitated by Res's pleiotropic effects. ${ }^{112}$ Patel et al. ${ }^{113,114}$ reported evidence that suggested that Res can be metabolized into stable sulfate-conjugated forms in human colorectal cells which in turn can provide beneficial effects in vivo in the form of generating parental compounds. 


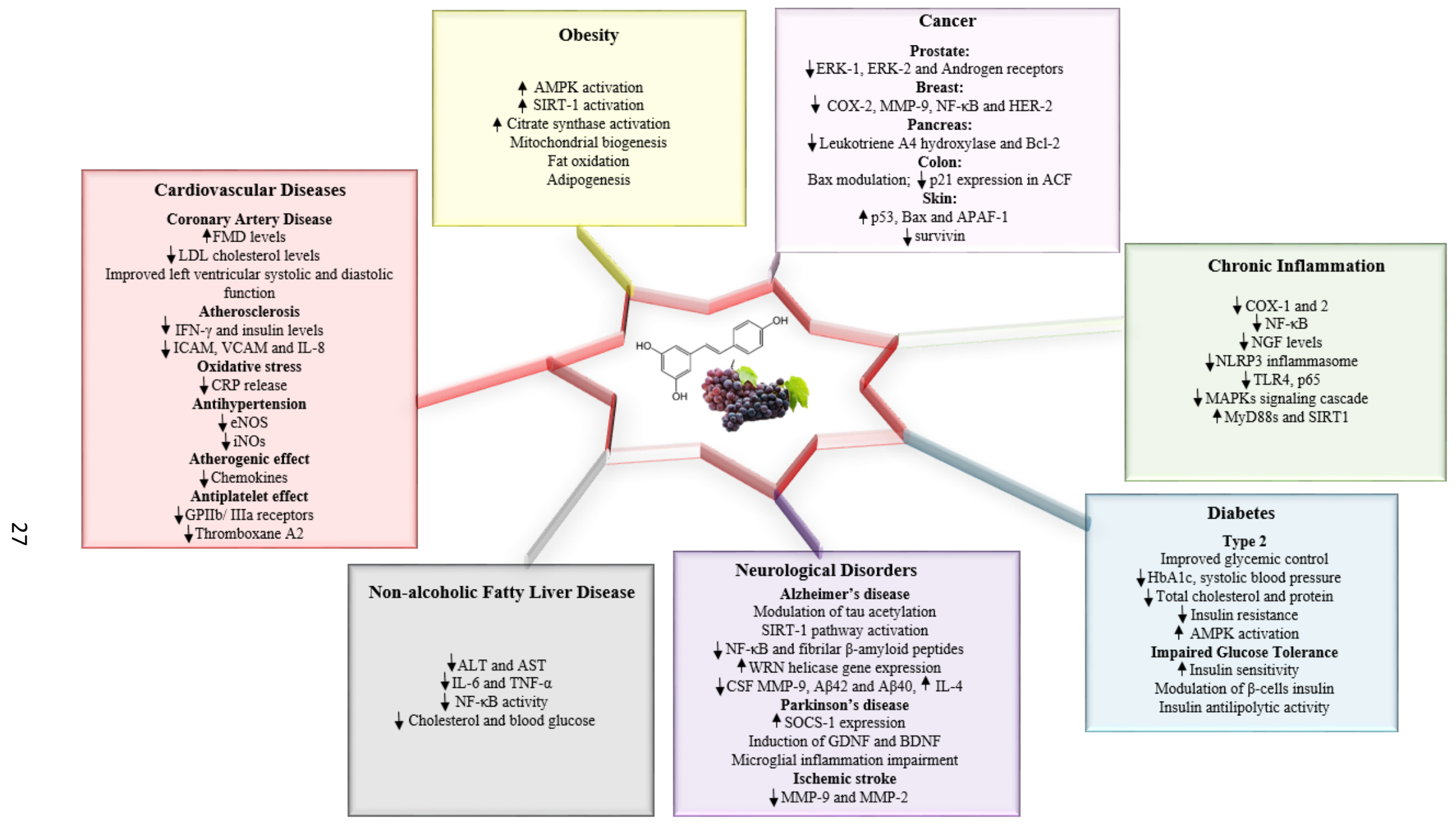

Figure 8: Clinical benefit of resveratrol in cancer, cardiovascular diseases, chronic inflammation, diabetes, neurological disorders and non-alcoholic fatty liver disease (Refer to the Abbreviation section for complete definition of the abbreviations). 


\subsubsection{Anticancer mechanisms of resveratrol bioactivity}

The chemical structure of Res is analogous to the structures of diethylstilbestrol, 17- $\beta$ estradiol and piceatannol, which are synthetic estrogen that can binds to the estrogen receptor alpha (ER $\alpha)$ as shown in Figure 9. ${ }^{115,116}$ This suggests that Res can act as an agonist of ER and thereby increase the transcription of estrogen-responsive reporter gene. ${ }^{15,117}$ Structural ligand-specific response insight into partial agonist and antagonist action of phytoestrogen Res on ER $\alpha$ ligand binding domain was further investigated by Chakraborty et al. ${ }^{118}$ using molecular modeling and atomistic simulations that confers chemoprotective properties in different cell types. Their findings revealed that Res can bind in both the antagonist and agonist conformations with ER $\alpha$ through the Helix 12 in the ligand binding pocket. ${ }^{118-120}$

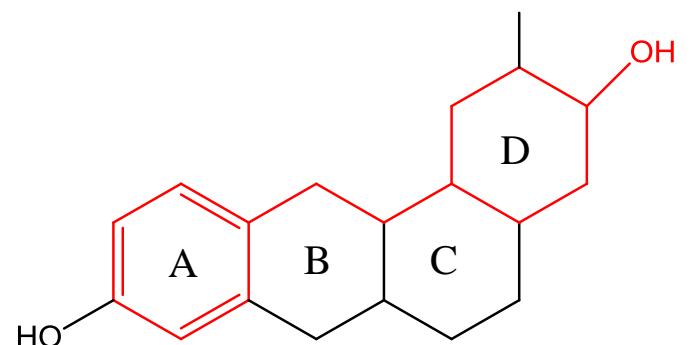

(i)

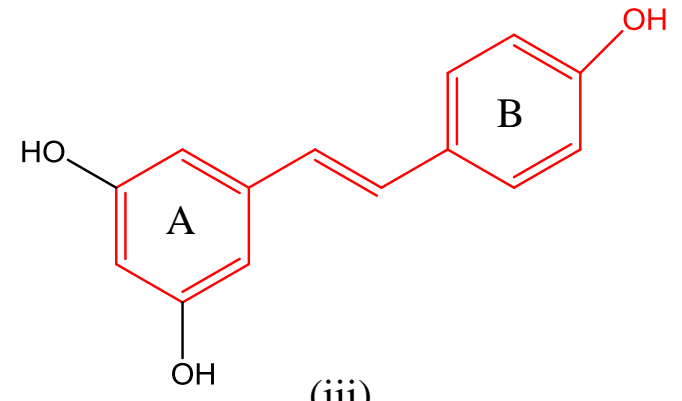

(iii)

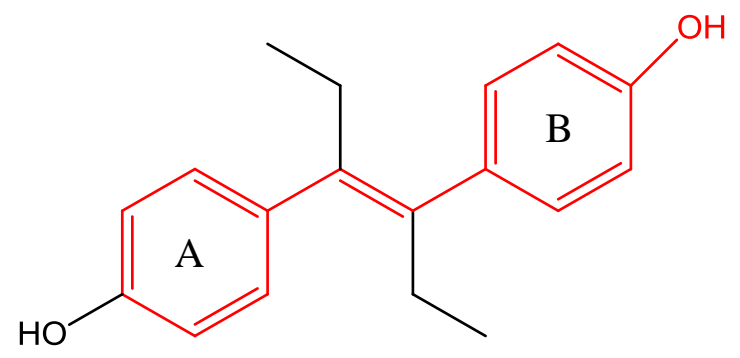

(ii)

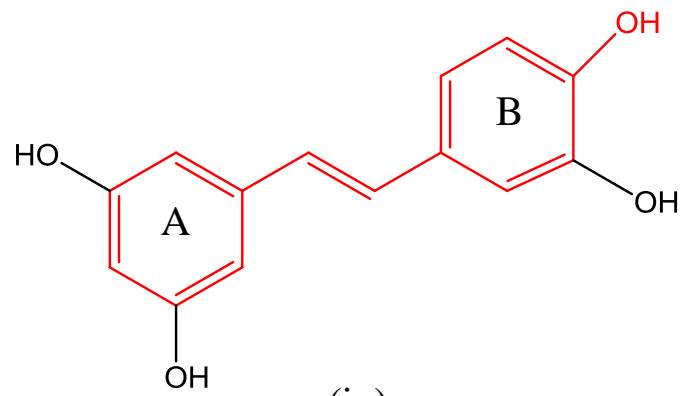

(iv)

Figure 9: Chemical structure of (i) 17- $\beta$ estradiol, (ii) Diethylstilbestrol, (iii) Resveratrol and (iv) Piceatannol, the red colored structure show the homology between these compounds. 
Studies by Uberti et al. ${ }^{121}$ and Cipolleti et al. ${ }^{122}$ demonstrated that Res binds to ER $\alpha$ and ER $\beta$ receptors through a biphasic effect on cell proliferation and suppressing growth. These results also showed that Res inhibited cell proliferation in ER-negative cancer cell lines. Walle et al. ${ }^{123}$ and Dandawate et al. ${ }^{124}$ reported that Res inhibits the proliferation of preneoplastic lesions by affecting signaling pathways through the activation of the de novo ceramide synthesis pathway. ${ }^{125}$ A number of human cancer cell lines (i.e. colon, breast, liver, lung, pancreatic, prostate and skin) against Res have been investigated and results showed the inhibition of cell proliferation, apoptosis induction and blocking cell cycle progression. ${ }^{126-128}$

Res was found to inhibit the transcriptional activation of the carcinogen-activating enzyme (CYP1A1), which is responsible for preventing cancer at the initiation stage according to reported by Ciolino et al. ${ }^{129}$ Res was reported to be a cyclooxygenase-2 (COX-2) inhibitor which is an enzyme induced during carcinogenesis and inflammation. ${ }^{120,130}$ Treatment with Res was shown to significantly decrease COX-2 expression related to the inhibition of nuclear factor kappa-light-chain-enhancer of activated B cells (NF-kB) and COX-2 has been suggested to play a role in p53-dependent apoptosis in cancer cells, thereby showing antitumor properties, conferring its chemopreventive properties. ${ }^{131,132}$

A number of studies have also reported Res to have antiaging properties, Pearson et al. ${ }^{133}$ and Bhullar et al. ${ }^{134}$ showed that elderly mice treated with Res had a beneficial effect in increasing their lifespan but not midlife mice. Kovacic and Somanathan ${ }^{135}$; Hsieh and $\mathrm{Wu}^{136}$ and Salehi et al. ${ }^{115}$ reported that the biological activities of Res were primarily attributed to the structural integrity of Res. The trans-Res with an ortho-diphenoxyl or para-diphenoxyl having a 4'hydroxyl and double bond showed a higher chemopreventive activity than the cis-Res 
configuration. ${ }^{137,138}$ Res has been shown to participate in the sirtuin (SIRT) pathway which regulates apoptosis, cell-cycle regulation (through caspase-3 activation and Bax upregulation), cellular differentiation and proliferation, DNA damage, immune responses and induces autophagy via the inhibition of the hosphatidylinositol-4,5-bisphosphate 3-kinase (PI3K)/Protein kinase B (AKT)/ mammalian target of rapamycin (mTOR) pathway. ${ }^{109,112,120}$ This was validated by Zhao et al. ${ }^{139}$ demonstrating that Res induces autophagy through the inhibition of STRT1/AMPK-mTOR pathway.

The laminin receptor modulates angiogenesis, enhanced invasive and metastatic potential of tumor cells (breast, prostate, lung, colon and etc.). Prostate tumor cells overexpress laminin receptors on their cell surface than normal cells and this can be exploited as a target for drugs. ${ }^{140}$ Res has been reported to target progesterone-responsive collagen-laminin receptor $\alpha 1$ integrin. Therefore laminin receptor can be a target molecule for Res. Han et al. ${ }^{141}$ demonstrated that the anticancer of Res analogs and epigallocatechin gallate (EGCG) could be mediated by the activation of common receptor binding sites.

Chemical metabolism and inhibitor for key proteins in signal transduction pathways (e.g. activator protein-1, mitogen-activated protein kinases (MAPK), cyclin dependent kinases, matrix metalloproteinases (MMPs), cyclins, AP-1, cytokines, COX-2 proteins and NF- $\kappa \mathrm{B}$ are facilitated by the antioxidant activity of Res. ${ }^{142-144}$ Elshaer et al. ${ }^{120}$ suggested that resveratrol-induced apoptosis occurs through a variety of p53-dependent and p53-independent pathways, expression of pro-apoptotic Bax with concomitant inhibition of anti-apoptotic Bc1-2 protein (Figure 10). Moreover, the mitochondrial pathway has been implicated by resveratrol-induced apoptosis via $\mathrm{Bax} / \mathrm{Bcl} 2$ ratio changes leading to the loss of mitochondrial transmembrane potential (MTP) with 
subsequent release of cytochrome $C$ followed by caspase cascade (caspase 3 and 9) results to apoptosis. ${ }^{128}$ The Pro-oxidant activity of Res is due to the hydroxylation that initiates a cellular apoptotic cascade through tumor necrosis factor (TNF)-related apoptosis-inducing ligand (TRAIL). ${ }^{145}$ Frazzi and Guardi ${ }^{112}$ mentioned that Res further participates within the mitochondrial apoptotic pathway which plays a crucial role during tumor death through changes in mitochondrial membrane potential leading in the subsequent release of cytochrome $\mathrm{c}$ in different tumor models. ${ }^{146}$ The epithelial-mesenchymal transition (EMT) plays a crucial role in tumorigenesis essential for metastasis, Res suppresses metastasis by modulating EMT-related factors (e.g. E-cadherin, N-cadherin, vimentin, MMP-2, and MMP-9) via the PI3K/Akt/NF- $\mathrm{B}$ signaling pathway. ${ }^{120,147}$

Catalgol et al. ${ }^{94}$ and Park et al. ${ }^{148}$ reported that Res regulates SIRT1 transcription and activity, this was further endorsed by Dai et al. ${ }^{149}$ who reported that Res is a therapeutic sirtuin activating compound for the overexpression of SIRT1 which inhibits several oncogenes and enzymes such as the cyclic adenosine monophosphate (cAMP)-degrading phosphodiesterases, resulting in induced apoptotic tumor suppression. ${ }^{149,150}$ Res is further involved in a cascade of pathways, as an activator for MAPK (MEK1/2) which catalyzes the phosphorylation of ERK1/2 which meditates cell proliferation and apoptosis. ${ }^{151,152}$ Res inhibits angiogenesis by suppressing the expression of vascular endothelial growth factor (VEGF), thereby downregulating hypoxiaInducible Factor $1 \alpha($ HIF-1 $\alpha) .{ }^{153}$ The onset of apoptosis can be triggered by the production of reactive oxygen species (ROS) and Res has been implicated in ROS production that seems to be a relevant mechanism of Res-induced cell death. ${ }^{154,155}$ 


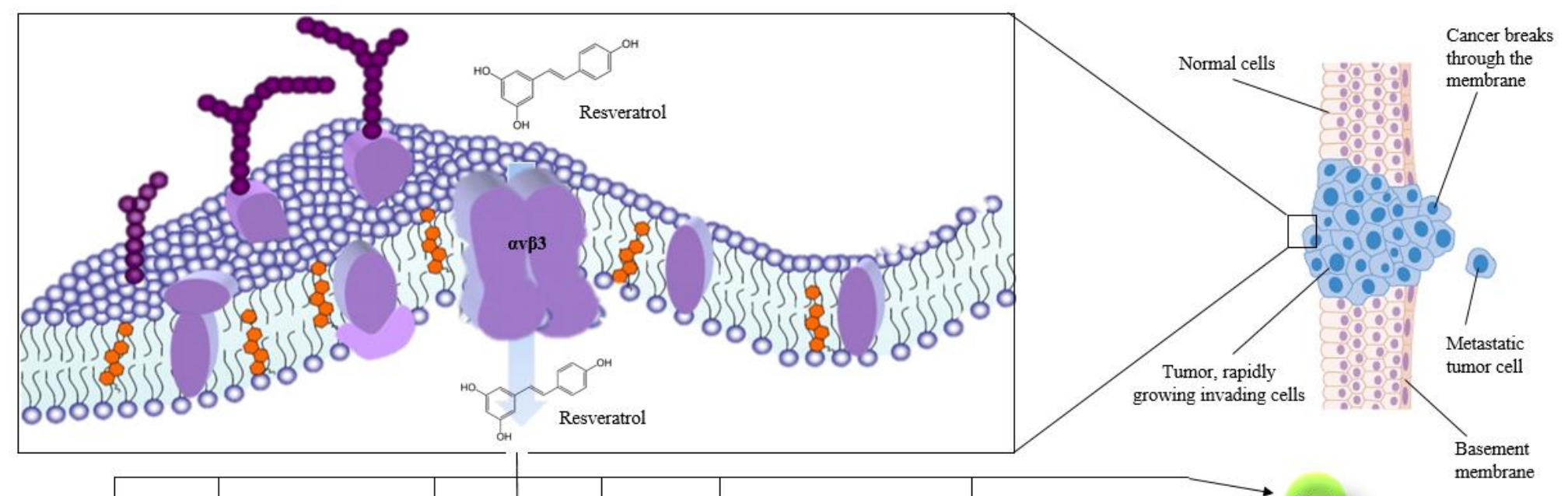

Figure 10: Schematic diagram that depicts the cancer therapeutic mechanism of resveratrol, inducing apoptosis through p53dependent activation of pro-apoptotic proteins (Bax, NOXA, and BUMA). Moreover, resveratrol upregulates Sirt1 and AMPK

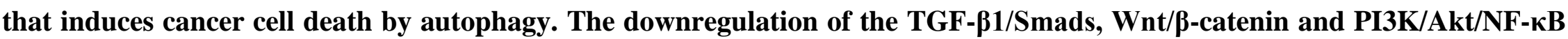
pathways by resveratrol inhibits EMT metastasis and HIF-1 $\alpha$ /MMPs dependent inhibits VEGF angiogenesis. 
In light of the reports of Res being a natural chemopreventive and chemotherapeutic agent against cancer and other-related diseases, this is supported by growing in vitro and in vivo data demonstrating the inhibitory effects of Res on a wide variety of human tumor cells. ${ }^{155}$ Antitumor and anti-metastatic properties of Res are due to the inhibition of DNA synthesis, neovascularization and angiogenesis. ${ }^{157,158}$ This data was corroborated by recent evidence demonstrating that cells undergo cell cycle arrest at the $\mathrm{G}_{0} / \mathrm{G}_{1}$ and $\mathrm{S}-\mathrm{G}_{2} / \mathrm{M}$ phase and apoptosis following Res treatment. ${ }^{12,159,160}$

\subsubsection{Resveratrol delivery through various mode of administration}

The cancer chemopreventative action of Res replies mainly on the delivery and bioavailability of Res to achieve a high therapeutic effectiveness. There are several routes of administration for Res:

(i) Buccal administration - Res absorption directly via the mucous membrane of the mouth. The drawback of this method is due to Res's low solubility which limits the absorbable amount through the buccal mucosa. ${ }^{159}$

(ii) Oral administration - as a tablet restricts the bioavailability because Res is metabolized rapidly into its glucuronate and sulfonate conjugates derivatives in the intestine and liver. ${ }^{161}$

Nanoemulsions are 20 to $200 \mathrm{~nm}$ formulations that are composed of two immiscible liquids that form a single phase through emulsification method. ${ }^{83}$ This mode of delivery enhances better retention for effective delivery of Res to the tumor. This can be achieved through the use of Liposomes which are spherical phospholipids composed of lipid bilayers as an advanced delivery 
system. Godin et al. ${ }^{162}$ reported the effective delivery of quercetin through the blood brain barrier for an increased therapeutic antitumor efficacy. Machida et al. ${ }^{163}$ and Sanli et al. ${ }^{164}$ demonstrated the delivery of Ginseng and curcumin phytosomes (phospholipid complexation) provided a better therapeutic efficacy attributed by elevated bioavailability and increased antioxidant activity. Microspheres (1 to $1000 \mu \mathrm{m}$ vesicular particles) have been reported as effective carriers for camptothecin for providing a prolonged anticancer effect. ${ }^{163-166}$ Transfersomal drug delivery of vincristine, tamoxifen, stavudine and interferon- $\alpha$ have been reported by Bhasin and Londhe. ${ }^{167}$

\subsubsection{Pitfalls and limitations associated with resveratrol delivery}

The stability of the formulation without compromising the anticancer therapeutic efficacy of Res can be a great challenge. The above-mentioned delivery systems must confine to release Res only at the site of delivery, thus a suitable system must take into consideration the pharmacodynamics and pharmacokinetics of the system. Insight about the pharmacokinetic mechanism can be performed through in vitro tests, however, the data do not provide any information about Res cellular uptake and metabolic responses ${ }^{83}$ Another major challenges is geared towards lack of recognition by pharmaceutical companies, industrial investments and federal funding in nutraceutical research, as nutraceutical formulations are not perceived as medicine ${ }^{85}$ Obeid et al. ${ }^{168}$ pharmaceutical companies have embraced synthetic and combinatorial technologies in favor of drug discovery programs based naturally derived candidate molecules.

We cannot conceal the body of evidence from research papers that have demonstrated the crucial role of nutraceuticals in the prevention and treatment of various diseases. Moreover, the incorporation of a nutraceutical such as resveratrol into a drug formulation serves as an effective 
alternative for patients who are unwilling to go through current chemotherapies (such as cisplatin) which affect the quality of life due to their toxicity. Thus, creating a paradigm shift in medicine relinquishing cancer management towards true health care focused at treating the root cause of the cancer rather than its systematic symptoms.

\subsection{Nanoscience and nanotechnology}

Nanoscience is the study of the properties of matter at a nanoscale $(1-100 \mathrm{~nm})$; it is an interdisciplinary and multidisciplinary field that integrates different knowledge and methods from various disciplines such as biology, chemistry, physics, materials science and engineering. Nanotechnology is the application of nanoscience through the design, manipulation and fabrication of materials by atom-by-atom or molecule-by-molecule while maintaining their shape and size at a nanoscale. This application offers materials with enhanced chemical and physical properties different from the same bulk material counterparts utilized in a variety of applications such as electronics, food agriculture, textiles, renewable energy, environmental, industrial, biomedical and healthcare sector.

\subsubsection{Nanotechnology in medicine}

Nanotechnologies utilized in the biomedical field have improved a myriad of applications for diagnostics and targeted therapy for drug delivery. This is due to the peculiar physiochemical properties of nanomaterials, which is attributed by increase surface-to-volume ratio. A variety of methods using hazardous substitutes (such as sodium borohydride, hydrazine) have been used to synthesize metallic nanoparticles with multifactorial interaction capabilities with biological systems that can result in toxic side effects impeding their clinical translation in the 
pharmaceutical and medical fields. The use of nanocarriers for delivery has demonstrated effectiveness in delivering a number of pro-drugs (doxorubicin). ${ }^{169}$

\subsubsection{Application of palladium nanoparticles (PdNPs)}

Palladium (Pd) is amongst the rare and precious metal (such as ruthenium, rhodium, osmium, iridium, and platinum) that belongs to the Platinum group elements. ${ }^{170}$ Moreover, $\mathrm{Pd}$ is predominately used as a catalyst in automotive catalytic converters, but recently has found applications in the electronic, engineering and biomedical sectors. ${ }^{171}$ Recent studies, have demonstrated Pd to have a propensity to adsorb hydrogen which has led palladium nanoparticles (PdNPs) to be utilized in hydrogen storage, catalysis and in fuel cells during electrochemical reactions. ${ }^{170,172,173}$ Different methods for palladium nanoparticles synthesis have been reported which include chemical reduction, metal vaporization, laser ablation, sonochemical and sol-gel process, however these methods generate hazardous byproducts and particles. ${ }^{172}$

Chitosan-graphene material doped with PdNPs have been used in the development of biosensors for determining of glucose levels. ${ }^{174}$ In medicine, Pd nanostructures have been reported as prodrug activator and photothermal agents for photothermal therapy (PTT) and as antitumor/antimicrobial agents, respectively. ${ }^{70}$ The PTT is through an external near-infrared (NIR) light source which emits a photon that penetrates through tissue to excites the tumor localized Pd nanostructures to produce heat, thereby causing tumor death as shown in Figure $11 .^{175-178}$ Combinational therapy using PdNPs with site-specific ligands as a NIR photo-absorber has a number of advantages such as precise spatial-temporal selectivity and effective eradication of tumor cells. Work by Rubio-Ruiz et al. ${ }^{179}$ demonstrated a high precision photothermal ablation using biocompatible PdNPs. 


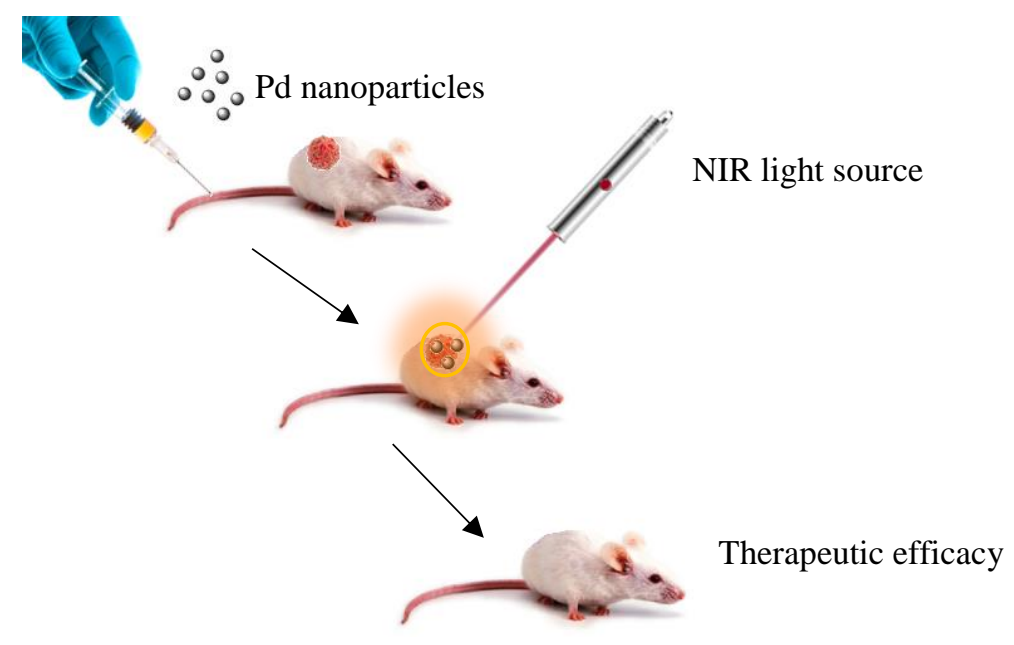

Figure 11: A schematic representation of PdNPs photothermal therapy, where the external NIR laser excites the PdNPs to generate heat for hyperthermia.

Rubio-Ruiz et al. ${ }^{179}$ further reported that NIR irradiated PdNPs conjugated with histidine significantly resulted in a higher temperature increase than PdNPs without histidine. This was postulated to histidine's aromatic ring thus increasing the NIR light absorption, which promoted their photothermal capabilities. ${ }^{180-182}$ Laser microscopy-patterned analysis revealed that squamous cell carcinoma SSC-GFP cells irradiated with NIR laser alone were not susceptible compared to cells labelled with PdNPs and NIR irradiated. ${ }^{179,183,184}$ An in vivo xenograft tissue ablation study by Rubio-Ruiz et al. ${ }^{179}$ on CD-1 nude mice showed that tumor xenograft composed of cancer cells with PdNPs induced photothermal tissue ablation.

Bharathiraja et al. $^{70}$ synthesized a multimodal complex (water-soluble chitosan coated biocompatible PdNPs conjugated with RGD (arginine-glycine-aspartic acid) peptide for photobased imaging and therapy. The results obtained showed that the RGD peptide-linked, chitosancoated PdNPs (Pd@Chitosan-RGD) enhanced photothermal therapeutic effects under 808 nm laser irradiation and even after 5-cycles of irradiation the Pd@Chitosan-RGD maintained their 
integrity. A vast pool of publications have demonstrated that PdNPs are ideal nanotheranostic candidates for enhanced photoacoustic imagining and photothermal therapy for tumors utilizing a non-invasive NIR laser. ${ }^{70,180}$ The benefit of NIR irradiation lies in water molecules having minimal absorbance around the NIR region, thereby preventing damage to healthy surrounding

tissues due to overheating. Reale et al. ${ }^{185}$ and Iavicoli et al. ${ }^{186}$ have reported that PdNPs further demonstrated to increase the levels of cytokines ( $\uparrow$ IFN- $\gamma$ release) as key mediators for their proinflammatory action.

\subsubsection{Palladium nanoparticles targeted cancer therapy}

\subsubsection{Toxicological effects of PdNPs}

Studies that investigate the toxicology of nanomaterials and PdNPs are on the cornerstone. The toxicity of PdNPs have been evaluated in vitro, ex vivo and in vivo models to assess a number of parameters which include cell viability, cytokine release, cell cycle dysfunction, oxidative stress reaction, DNA damage and the release of inflammatory mediators as shown in Table 4. 
Table 4: Toxicology of palladium nanoparticles

\begin{tabular}{|c|c|c|c|c|}
\hline $\begin{array}{l}\text { Type of } \\
\text { PdNPs }\end{array}$ & Experimental design & $\begin{array}{c}\text { Parameters } \\
\text { investigated }\end{array}$ & Results & Reference \\
\hline \multicolumn{5}{|c|}{ In vitro } \\
\hline $1-12 \mathrm{~nm}$ & $\begin{array}{l}\text { Barley (Hordeum } \\
\text { vulgare) seeds were } \\
\text { cultivated in a } \\
\text { hydroponic system } \\
\text { containing a nutrient } \\
\text { solution supplemented } \\
\text { with } 0-50 \mu \mathrm{M} \text { PdNPs } \\
\text { for 1 week. }\end{array}$ & Leaf length & $\begin{array}{l}\text { Leaf length: } \downarrow \text { at } \\
\geq 40 \mu \mathrm{M} \text { of PdNPs } \\
\text { compared to } \\
\text { untreated plants } \\
\text { (length: } 115 \mathrm{~mm} \mathrm{vs} \\
125 \quad \mathrm{~mm} \text {, } \\
\text { respectively). }\end{array}$ & Battke et al. ${ }^{187}$ \\
\hline $\begin{array}{l}\mathrm{Pd} / \text { magnetite } \\
\text { nanoparticles } \\
(20-30 \mathrm{~nm})\end{array}$ & $\begin{array}{l}\text { Rainbow trout gills } \\
\text { (RTgill-W1) cells were } \\
\text { treated with } 5-25 \mathrm{mg} / \mathrm{L} \\
\text { PdNPs for } 72 \mathrm{~h} .\end{array}$ & Cell viability & $\begin{array}{l}\text { Metabolic activity } \\
\text { and membrane } \\
\text { integrity: } \downarrow \text { after } 1 \\
h, \text { however, cell } \\
\text { viability was fully } \\
\text { restored after } 72 \mathrm{~h} \text {. }\end{array}$ & $\begin{array}{l}\text { Potthoff et } \\
\text { al. }\end{array}$ \\
\hline
\end{tabular}




\section{In vivo}

\begin{tabular}{|c|c|c|c|c|}
\hline \multirow{2}{*}{$10 \pm 6 \mathrm{~nm}$} & $\begin{array}{l}\text { Female Wistar rats (n. } \\
5 \text { per group) were } \\
\text { treated with a single } \\
\text { intravenous injection } \\
\text { of } 0-12 \mu \mathrm{g} / \mathrm{kg} \text { PdNPs } \\
\text { dose from day } 1,30 \& \\
60 . \text { Serum cytokines } \\
\text { were assessed after the } \\
90^{\text {th }} \text { day post exposure. }\end{array}$ & $\begin{array}{l}\text { Effects on } \\
\text { the immune } \\
\text { system }\end{array}$ & $\begin{array}{l}\text { Treatment at } 12 \\
\mu \mathrm{g} / \mathrm{kg} \text { PdNPs: } \downarrow \\
\text { IL-1 } \alpha, \text { IL-4, IL-6, } \\
\text { IL-10, IL-12 and } \\
\text { GM-CSF }\end{array}$ & $\begin{array}{l}\text { Iavicoli et } \\
\text { al. }^{186}\end{array}$ \\
\hline & $\begin{array}{l}\text { Female Wistar rats (n. } \\
4 \text { per group) were } \\
\text { treated with a single } \\
\text { intravenous injection } \\
\text { of } 0-12 \mu \mathrm{g} / \mathrm{kg} \text { PdNPs. } \\
\text { Sex hormones (E2, } \\
\text { FSH, LH, P and T) } \\
\text { levels were analyzed } \\
\text { after } 14 \text { days of } \\
\text { exposure. }\end{array}$ & $\begin{array}{l}\text { Effects on } \\
\text { the } \\
\text { endocrine } \\
\text { system }\end{array}$ & $\begin{array}{l}\text { Treatment at } 12 \\
\mu \mathrm{g} / \mathrm{kg} \text { PdNPs: } \downarrow \text { E2 } \\
\text { and } \mathrm{T} \text { while, } \uparrow \\
\text { FSH, LH, and } \mathrm{P} \\
\text { levels }\end{array}$ & Leso et al. ${ }^{189}$ \\
\hline
\end{tabular}

E2, estradiol; FSH, follicle-stimulating hormone; GM-CSF, granulocyte-macrophage colony stimulating factor; IL, interleukin; LH, luteinizing hormone; $\mathrm{P}$, progesterone; T, testosterone. 
The results mentioned in Table 4 are based on studies that produced the tested PdNPs using chemical reagents such as sodium borohydride $\left(\mathrm{NaBH}_{4}\right)$ as the reducing agent. Thereby, producing PdNPs that are not biocompatible increases human health and environmental risks. To overcome this challenge, alternative methods of synthesis are required, hence Green Nanotechnology a technology used to develop ecofriendly biocompatible materials to minimize human health and potential environmental risks. 


\section{REFERENCES}

1. Golemis EA, Scheet P, Beck TN, Scolnick EM, Hunter DJ, Hawk E, Hopkins N. Molecular mechanisms of the preventable causes of cancer in the United States. Genes Dev. 2018:1-35. doi:10.1101/gad.314849.118.

2. Biemar F, Foti M. Global progress against cancer - challenges and opportunities. Cancer Biol Med. 2013:183-186. doi:10.7497/j.issn.2095-3941.2013.04.001.

3. DeVita VT, Chu E. A History of Cancer Chemotherapy.eview article. Cancer Res. 2008; 68: (21).

4. Levine MS, Holland AJ. The impact of mitotic errors on cell proliferation and tumorigenesis. Genes and Dev. 2015:620-638. doi:10.1101/gad.314351.118.620.

5. Makridakis N, Reichardt JKV. Pharmacogenetic analysis of human steroid 5 reductase type II : comparison of finasteride and dutasteride. J Mol Endocrinol. 2004:617-623. doi:10.1677/jme.1.01725.

6. Siegel RL, Miller KD, Jemal A. Cancer statistics. CA Cancer J Clin. 2017;67(1):7-30. doi: 10.3322/caac. 21387 .

7. World Health Organization. Global action plan for the prevention and control of noncommunicable diseases 2013-2020. World Heal Organ. 2013:102. doi:978 924 1506236.

8. International Agency for Research on Cancer (IARC). Online: https://www.iarc.fr/wpcontent/uploads/2018/09/pr263_E.pdf. (Accessed September 2018). 
9. Bray F, Ferlay J, Soerjomataram I. Global Cancer Statistics 2018: GLOBOCAN Estimates of Incidence and Mortality Worldwide for 36 Cancers in 185 Countries. $C A$ Cancer J Clin. 2018:394-424. doi:10.3322/caac.21492.

10. Weir HK, Thompson TD, Soman A, Møller B, Leadbetter S. The past, present, and future of cancer incidence in the United States: 1975 through 2020. Cancer. 2015;121(11):18271837. doi:10.1002/cncr.29258.

11. Siegel RL, Miller KD, Jemal A. Cancer statistics. CA Cancer J Clin. 2018;68(1):7-30. doi:10.3322/caac.21442.

12. Siegel RL, Miller KD, Jemal A. Cancer statistics. CA Cancer J Clin. 2019;69(1):7-34. doi:10.3322/caac.21551.

13. Mariotto AB, Robin Yabroff K, Shao Y, Feuer EJ, Brown ML. Projections of the cost of cancer care in the United States: 2010-2020. J Natl Cancer Inst. 2011;103(2):117-128. doi:10.1093/jnci/djq495.

14. Yabroff KR, Lund J, Kepka D, Mariotto A. Economic burden of cancer in the United States: estimates, projections, and future research. Cancer Epidemiol Biomarkers Prev. 2011;20(10):2006-2014. doi:10.1158/1055-9965.EPI-11-0650.

15. Fleming KA, Naidoo M, Wilson M, et al. An Essential Pathology Package for Low- and Middle-Income Countries. 2017:15-32. doi:10.1093/AJCP/AQW143.

16. Sayed S, Cherniak W, Lawler M, Tan SY, El Sadr W, Wolf N, Silkensen S, Brand N, Looi LM, Pai SA, Wlison ML, Milner D, Flanigan J, Fleming KA. Pathology and 
laboratory medicine in low-income and Improving pathology and laboratory medicine in low-income and middle-income countries: roadmap to solutions. Lancet. 2018;391(10133):1939-1952. doi:10.1016/S0140-6736(18)30459-8.

17. Prager GW, Braga S, Bystricky B, Qvortrup C, Criscitiello C, Esin E, Sonke GS, Martínez GA, Frenel JS, Karamouzis M, Strijbos M, Yazici O, Bossi P, Banerjee S, Troiani T, Eniu A, Ciardiello F, Tabernero J, Zielinski CC, Casali PG, Cardoso F, Douillard JY, Jezdic S, McGregor K, Bricalli G, Vyas M, Ilbawi A. Global cancer control : responding to the growing burden, rising costs and inequalities in access. ESMO Open. 2018:1-10. doi:10.1136/esmoopen-2017-000285.

18. Howe R, Hassett MJ, Wheelock A, Donoghue CO, Kaplan C, Ozanne EM. Costs matter : The impact of disclosing treatment costs and provider profit on patients' decisions. $J$ Cancer Policy. 2017;11:42-47. doi:10.1016/j.jcpo.2016.09.002.

19. Fizazi K, Smith MR, Tombal B. Clinical Development of Darolutamide: A Novel Androgen Receptor Antagonist for the Treatment of Prostate Cancer. Clin Genitourin Cancer. 2018;16(5):332-340. doi:10.1016/j.clgc.2018.07.017.

20. Chiou Y, Li S, Ho C, Pan M. Prevention of Breast Cancer by Natural Phytochemicals: Focusing on Molecular Targets and Combinational Strategy. Mol Nutr Food Res. 2018; 62(23):e1800392 . doi:10.1002/mnfr.201800392.

21. Farkona S, Diamandis EP, Blasutig IM. Cancer immunotherapy : the beginning of the end of cancer ? BMC Med. 2016:1-18. doi:10.1186/s12916-016-0623-5. 
22. Messerschmidt JL, Prendergast GC, Messerschmidt GL. How Cancers Escape Immune Destruction and Mechanisms of Action for the New Significantly Active Immune Therapies: Helping Nonimmunologists Decipher Recent Advances. Oncologist. 2016;21(2):233-243.

23. Kroemer G, Zitvogel L. The breakthrough of the microbiota. Nat Publ Gr. 2018;18(2):8788. doi:10.1038/nri.2018.4.

24. Kowalewski D, De T, Rammensee HG, Stevanovic S. Hillin. Vol 2.; 2018.

25. Sahin U, Türeci Ö. Personalized vaccines for cancer immunotherapy. Science 2018;359(6382):1355-1360. doi: 10.1126/science.aar7112.

26. Schulz-Knappe PK, Lautscham G. Prevent Cancer Immunotherapy's Side Effects. Genet Eng Biotechnol News. 2018;38(12):28-29. doi:10.1089/gen.38.12.12.

27. Tang J, Pearce L, Donnell-tormey JO, Hubbard-lucey VM. FROM THE ANALYST' S COUCH Trends in the global immuno-oncology landscape. Nat Publ Gr. 2018;17(11):783-784. doi:10.1038/nrd.2018.167.

28. Dolgin E. Cancer's cost conundrum. Nature. 2018;555:S26-S29. doi:10.1200/JCO.2016.72.2124.

29. Ferlay J, Colombet M, Soerjomataram I, Mathers C, Parkin DM, Piñeros M, Znaor A, Bray F. Estimating the global cancer incidence and mortality in 2018: GLOBOCAN sources and methods. Int J Cancer. 2018. doi:10.1002/ijc.31937. 
30. Fontana F, Raimondi M, Marzagalli M, Sommariva M, Limonta P, Gagliano N. Epithelial-To-Mesenchymal Transition Markers and CD44 Isoforms Are Differently Expressed in 2D and 3D Cell Cultures of Prostate Cancer Cells. Cells. 2019;8(2):143. doi:10.3390/cells8020143.

31. Siegel RL, Miller KD. Cancer Statistics , 2019. 2019;69(1):7-34. doi:10.3322/caac.21551.

32. Weir HK, Thompson TD, Soman A, Møller B. The Past, Present, and Future of Cancer Incidence in the United States: 1975 Through 2020. 2020:1827-1837. doi:10.1002/cncr.29258.

33. Mazhar D, Waxman J. Prostate cancer. 2002;(July):590-595.

34. Zhou J, Yang C, Lu Z, Zhang L, Yin Y, Tai S, Liang C. Primary urothelial carcinoma of the prostate. Medicine (Baltimore). 2019;98(3):1-5.

35. Angulo JC, Núñez C, Gonzalez J, Hernández E, Castillo E, Rodríguez-Barbero JM. Primary transitional cell carcinoma of the prostate: a male disease with dismal prognosis despite cisplatin-based systemic chemotherapy. J Mens health. 2010;7(1):64-72. doi:https://doi.org/10.1016/j.jomh.2009.12.004.

36. Malik RD, Dakwar G, Hardee ME, Sanfilippo NJ, Rosenkrantz AB, Taneja SS. Squamous Cell Carcinoma of the Prostate. Rev Urol. 2011;13(1):56-60. doi:10.3909/riu0494.

37. Biswas T, Podder T, Lepera PA, Walker P. Primary squamous cell carcinoma of the prostate : a case report of a rare clinical entity. Future Sci OA. 2015;1:5-10. 
38. Brunnhoelzl D, Wang J. Clinical features, treatment, prognosis, and outcome of 47 patients with pure squamous cell carcinoma of the prostate. $J$ Clin Oncol. 2018;36(6_suppl):7.doi:10.1200/JCO.2018.36.6_suppl.7.

39. Nadal R, Street NB, Eisenberger MA. Small cell carcinoma of the prostate. Nat Rev Urol. 2015;11(4):213-219. doi:10.1038/nrurol.2014.21.Small.

40. Weprin S, Yonover P. Urology Case Reports Small Cell Carcinoma of the Prostate : A Case Report and Brief Review of the Literature. Urol Case Reports. 2017;13:61-62. doi:10.1016/j.eucr.2016.10.010.

41. Bouchelouche K, Choyke PL, Capala J. Prostate specific membrane antigen- a target for imaging and therapy with radionuclides. Discov Med. 2010;9(44):55-61. doi:10.1097/MPG.0b013e3181a15ae8.Screening.

42. Dong DX, Ji ZG. Current progress and controversies in prostate cancer management. Chin Med J (Engl). 2017;130(24):2991-2995. doi:10.4103/0366-6999.220317.

43. Litwin MS, Tan HJ. The diagnosis and treatment of prostate cancer: A review. JAMA - J Am Med Assoc. 2017;317(24):2532-2542. doi:10.1001/jama.2017.7248.

44. Kearns JT, Holt SK, Wright JL, Lin DW, Lange PH, Gore JL. PSA screening, prostate biopsy, and treatment of prostate cancer in the years surrounding the USPSTF recommendation against prostate cancer screening. Cancer. 2018;124(13):2733-2739. doi:10.1002/cncr.31337.

45. Tian JY, Guo FJ, Zheng GY, Ahmad A. Prostate cancer: Updates on current strategies for 
screening, diagnosis and clinical implications of treatment modalities. Carcinogenesis. 2018;39(3):307-317. doi:10.1093/carcin/bgx141.

46. Crawford ED, Petrylak D, Sartor O. Navigating the evolving therapeutic landscape in advanced prostate cancer. Urol Oncol Semin Orig Investig. 2017;35:S1-S13. doi:10.1016/j.urolonc.2017.01.020.

47. Ramroop JR, Stein MN, Drake JM. Impact of Phosphoproteomics in the Era of Precision Medicine for Prostate Cancer. Front Oncol. 2018;8(February). doi:10.3389/fonc.2018.00028.

48. Filson CP, Marks LS, Litwin MS. Expectant management for men with early stage prostate cancer. CA Cancer J Clin. 2015;65(4):264-282. doi:10.3322/caac.21278.

49. Dickens E, Ahmed S. Principles of cancer treatment by chemotherapy. Surg (United Kingdom). 2018;36(3):134-138. doi:10.1016/j.mpsur.2017.12.002.

50. Karadurmuş N, Ertürk İ. Which is the best for cancer treatment? Surgery or chemotherapy. J Oncol Sci. 2017;3(1):32-33. doi:10.1016/j.jons.2017.02.001.

51. Benjamin DJ. The efficacy of surgical treatment of cancer - 20 years later. Med Hypotheses. 2014;82(4):412-420. doi:10.1016/j.mehy.2014.01.004.

52. Sgouros G, Goldenberg DM. Radiopharmaceutical therapy in the era of precision medicine. Eur J Cancer. 2014;50(13):2360-2363. doi:10.1016/j.ejca.2014.04.025.

53. Goel AK, De S. Abiraterone acetate, an inhibitor of adrenal androgen synthesis in 
"hormone refractory prostate cancer." Indian J Med Paediatr Oncol. 2011;32(1):43-45. doi:10.4103/0971-5851.81890.

54. S. Matos C, L.M.B. de Carvalho A, P. Lopes R, P.M. Marques M. New Strategies Against Prostate Cancer - Pt(II)-Based Chemotherapy. Curr Med Chem. 2012;19(27):4678-4687. doi:10.2174/092986712803306394.

55. Sundararajan S, Vogelzang N. Chemotherapy in the Treatment of Prostate Cancer - The Past, the Present, and the Future. Chemotherapy. 2014;10(6):14-21.

56. Anassi E, Ndefo UA. Sipuleucel-T (provenge) injection: the first immunotherapy agent (vaccine) for hormone-refractory prostate cancer. P T. 2011;36(4):197-202.

57. Bilen MA, Hess KR, Subudhi SK, Aparicio A, Kim J, Zurita-Saavedra AJ, Araujo JC, Corn PG, Stover J, Lin SH, Logothetis CJ, Tu SM. Clinical predictors of survival in patients with castration-resistant prostate cancer receiving sipuleucel-T cellular immunotherapy. Cancer Chemother Pharmacol. 2017;80(3):583-589. doi:10.1007/s00280-017-3391-9.

58. Jahromi EZ, Divsalar A, Saboury AA, Khaleghizadeh S, Mansouri-Torshizi H, Kostova I. Palladium complexes: new candidates for anti-cancer drugs. J Iran Chem Soc. 2018;13(5):967-989. doi:10.1007/s13738-015-0804-8.

59. Pages BJ, Garbutcheon-singh KB, Aldrich-wright JR. Platinum Intercalators of DNA as Anticancer Agents. Eur. J. Inorg. Chem. 2017:1613-1624. doi:10.1002/ejic.201601204.

60. Fong CW. Platinum anti-cancer drugs: free radical mechanism of Pt-DNA adduct 
formation and anti-neoplastic effect . Free Radic Biol Med. 2016;95:216-29. doi: 10.1016/j.freeradbiomed.2016.03.006.

61. Johnston A, Holt DW. Substandard drugs: a potential crisis for public health. Br J Clin Pharmacol. 2013;78(2):218-243. doi:10.1111/bcp.12298.

62. Iqbal J, Abbasi BA, Mahmood T, Kanwal S, Ali B, Shah AS, Khalil T. Plant-derived anticancer agents: A green anticancer approach. Asian Pac J Trop Biomed. 2017;7(12):1129-1150. doi:10.1016/j.apjtb.2017.10.016.

63. Aston WJ, Hope DE, Nowak AK, Robinson BW, Lake RA, Lesterhuis WJ. A systematic investigation of the maximum tolerated dose of cytotoxic chemotherapy with and without supportive care in mice. BMC Cancer. 2017:1-10. doi:10.1186/s12885-017-3677-7.

64. Gao E, Zhu M, Yin H, Liu L, Wu Q, Sun Y. Synthesis, characterization, interaction with DNA and cytotoxicity in vitro of dinuclear Pd(II) and Pt(II) complexes dibridged by 2,2'azanediyldibenzoic acid. $J$ Inorg Biochem. 2008;102(10):1958-1964. doi:10.1016/j.jinorgbio.2008.07.011.

65. Abu-surrah AS, Al-sa HH, Abdalla MY. Palladium-based chemotherapeutic agents: Routes toward complexes with good antitumor activity. Cancer Therapy. 2008;6:1-10.

66. Gao E, Zhu M, Yin H, Liu L, Wu Q, Sun Y. Synthesis, characterization, interaction with DNA and cytotoxicity in vitro of dinuclear Pd(II) and Pt(II) complexes dibridged by 2,2'azanediyldibenzoic acid. $J$ Inorg Biochem. 2008;102(10):1958-1964. doi:10.1016/j.jinorgbio.2008.07.011. 
67. Ghobadi R, Divsalar A, Reza A, Akbar A. Spectroscopic investigation of Bovine Liver Catalase interactions with a novel phen-imidazole derivative of platinum. J Biomol Struct Dyn. 2018;36(3):656-662. doi: 10.1080/07391102.2017.1290551.

68. Choi KW, Kim DY, Ye SJ, Park OO. Shape- and Size-Controlled Synthesis of Noble Metal Nanoparticles. Adv Mat Res. 2014;3(4) 129-137

69. Torborg C, Beller M. Recent Applications of Palladium-Catalyzed Coupling Reactions in the Pharmaceutical, Agrochemical, and Fine Chemical Industries. Adv. Synth. Cata. 2009. doi:10.1002/adsc.200900587.

70. Bharathiraja S, Bui NQ, Manivasagan P, Moorthy MS, Mondal S, Seo H, Phuoc NT, Vy Phan TT, Kim H, Lee KD, Oh J. Multimodal tumor-homing chitosan oligosaccharidecoated biocompatible palladium nanoparticles for photo-based imaging and therapy. Sci Rep. 2018:1-16. doi:10.1038/s41598-017-18966-8.

71. Gama NH, Elkhadir AYF, Gordhan BG, Kana BD, Darkwa J, Meyer D. Activity of phosphino palladium(II) and platinum(II) complexes against HIV-1 and Mycobacterium tuberculosis. BioMetals. 2016;29(4):637-650.

72. Skowronek PJ. Current status of brachytherapy in cancer treatment - short overview. J Contemp Brachytherapy. 2017;9(6). doi: 10.5114/jcb.2017.72607.

73. Brumm J. Brachytherapy as a treatment option for prostate cancer : overview and nursing considerations. Proc (Bayl Univ Med Cent). 2000:227-229.

74. Huq F, Tayyem H, Beale P, Yu JQ. Studies on the activity of three palladium ( II ) 
compounds of the form: Trans -PdL $2 \mathrm{Cl} 2$ where $\mathrm{L}=2$-hydroxypyridine. J. Inorg. Biochem. 2007;101:30-35. doi:10.1016/j.jinorgbio.2006.08.001.

75. Zhao G, Sun H, Lin H, Zhu S, Su X, Chen Y. Palladium(II) complexes with N,N'-Dialkyl1,10-phenanthroline-2,9-dimathanamine: synthesis, characterization and cytotoxic activity. J Inorg Biochem. 1998;72(3):173-177. doi:https://doi.org/10.1016/S01620134(98)10077-6.

76. Rodrigues EG, Silva LS, Fausto DM, Hayashi MS, Dreher S, Santos EL, Pesquero JB, Travassos LR, Caires ACF. Cyclopalladated compounds as chemotherapeutic agents: Antitumor activity against a murine melanoma cell line. Int J Cancer. 2003;107(3):498504. doi:10.1002/ijc.11434.

77. Arora S, Agarwal S, Singhal S. Anticancer activities of thiosemicarbazides/thiosemicarbazones: A review. Int J Pharm Pharm Sci. 2014;6(9).

78. Cheng H, Huq F, Beale P, Fisher K. Synthesis, characterisation, activities, cell uptake and DNA binding of a trinuclear complex: [[trans-PtCl(NH3)]2mu-[trans-Pd(NH3)(2hydroxypyridine)-(H2)N(CH2)6NH2)2]Cl4. Eur. J. Med. Chem. 2006;41:896-903. doi:10.1016/j.ejmech.2006.03.026.

79. El-Sherif AA (2012). Coordination Chemistry of Palladium(II) Ternary Complexes with Relevant Biomolecules, Stoichiometry and Research - The Importance of Quantity in Biomedicine, Dr Alessio Innocenti (Ed.), ISBN: 978-953-51-0198-7, InTech, Available from: http://www.intechopen.com/books/stoichiometry-andresearch-the-importance-ofquantity-in-biomedicine/coordination-chemistry-of-palladium-ii-ternary-complexeswith- 
relevant-biomolecules

80. Marques MPM. Platinum and Palladium Polyamine Complexes as Anticancer Agents: The Structural Factor. Int Sch Res Notices. 2013;1-29. doi.org/10.1155/2013/287353

81. Zhang P, Sadler. Advances in the design of organometallic anticancer complexes. J. Organomet. Chem. 2017;839(15):5-14. https://doi.org/10.1016/j.jorganchem.2017.03.038.

82. Krylova LF, Kovtunova LM, Romanenko G V. Pt ( II ) and Pd ( II ) Complexes with $\beta$ Alanine. Bioinorg Chem Appl. 2008. doi:10.1155/2008/983725.

83. Ruchi S, Amanjot K, Sourav T, Keerti B, Sujit B. Role of nutraceuticals in health care: A review. IJGP. 2017;2017(3):2-8. doi.org/10.22377/ijgp.v11i03.1146.

84. Liang J, Bi N, Wu S, Chen M, Lv C, Zhao L, Shi A, Jiang W, Xu Y, Zhou Z, Wang W, Chen D, Hui Z, Lv J, Zhang H, Feng Q, Xiao Z, Wang X, Liu L, Zhang T, Du L, Chen W, Shyr Y, Yin W, Li J, He J, Wang L. Etoposide and cisplatin versus paclitaxel and carboplatin with concurrent thoracic radiotherapy in unresectable stage III non-small cell lung cancer: a multicenter randomized phase III trial. Ann Oncol. 2017;28(4):777-783. doi: 10.1093/annonc/mdx009.

85. Ramaswamy S. Reflections on current Ayurveda research. J Ayurveda Integr Med. 2018;9:250-251. doi:10.1016/j.jaim.2018.11.001.

86. Iqbal J, Abbasi BA, Mahmood T, Kanwal S, Ali B, Shah SA, Khalil AT. Plant-derived anticancer agents: A green anticancer approach. Asian Pac J Trop Biomed. 2017;7(12):1129-1150. doi:10.1016/j.apjtb.2017.10.016. 
87. Weaver BA. How Taxol/paclitaxel kills cancer cells. Mol Biol Cell. 2014;25(18):26772681. doi:10.1091/mbc.E14-04-0916.

88. Pandey N, Meena RP, Rai SK, Rai SP. Medicinal plants derived nutraceuticals: A Reemerging health aid. Int J Pharma Bio Sci. 2011;2(4):419-441.

89. Holland JF, Scharlau C, Gailani S, Krant MJ, Olson KB, Horton J, Shnider BI, Lynch JJ, Owens A, Carbone PP, Colsky J, Grob D, Miller SP, Hall TC. Vincristine Treatment of Advanced Cancer: A Cooperative Study of 392 Cases. Cancer Res. 1973;33(6):1258 LP1264.

90. Rajat S, Manisha S, Robin S, Sunil K. Nutraceuticals: a Review. IRJP. 2012;3(4):95-99.

91. Venditto VJ, Simanek EE. Cancer therapies utilizing the camptothecins: a review of the in vivo literature. Mol Pharm. 2010;7(2):307-349. doi:10.1021/mp900243b.

92. Kim YH, Mio T, Hamamoto Y, Mishima M. Successful treatment with carboplatin and etoposide in a small-cell lung cancer patient undergoing hemodialysis. Respir Med CME. 2010;3(2):68-70. doi:https://doi.org/10.1016/j.rmedc.2009.05.006.

93. FDA. Epidiolex prescribing information. Onlline: https://www.accessdata.fda.gov/drugsatfda_docs/label/2018/210365lbl.pdf. （Accessed December, 2018)

94. Catalgol B, Batirel S, Taga Y, Ozer NK. Resveratrol: French Paradox Revisited. Front Pharmacol. 2012. doi:10.3389/fphar.2012.00141. 
95. Haseeb S, Alexander B, Baranchuk A. Wine and Cardiovascular Health. Circulation. 2017:1434-1448. doi:10.1161/CIRCULATIONAHA.117.030387.

96. Gaetano G De, Castelnuovo A, Rotondo S. Cardiovascular protective effect of moderate wine consumption: evidence after the French Paradox. Sang Thrombose Vaisseaux. 2005;17:47-60.

97. Stockley CS. The relationships between alcohol, wine and cardiovascular diseases - A review. J Nutr Health Aging. 2015;3:55-88. doi:10.3233/NUA-150052.

98. Prasad K, . Resveratrol, Wine, and Atherosclerosis. Int J Angiol. 2012;1(212). doi: $10.1055 / \mathrm{s}-0032-1306417$.

99. Zordoky BNM, Robertson IM, Dyck JRB. Preclinical and clinical evidence for the role of resveratrol in the treatment of cardiovascular diseases. Biochim Biophys Acta. 2015;1852(6):1155-1177. doi:10.1016/j.bbadis.2014.10.016.

100. Jahnert T, Hager MD, Schubert US. Application of phenolic radicals for antioxidants, as active materials in batteries, magnetic materials and ligands for metal-complexes . $J$. Mater. Chem. A. 2014:15234-15251. doi:10.1039/c4ta03023k.

101. Timmers S, Auwerx J, Schrauwen P. The journey of resveratrol from yeast to human. Aging (Albany NY). 2012;4(3):146-158.

102. Coimbra M, Isacchi B, van Bloois L, Torano JS, Ket A, Wu X, Broere F, Metselaar JM, Rijcken CJ, Storm G, Bilia R, Schiffelers RM. Improving solubility and chemical stability of natural compounds for medicinal use by incorporation into liposomes. Int $J$ 
Pharm. 2011;416(2):433-442. doi:https://doi.org/10.1016/j.ijpharm.2011.01.056.

103. Ortuño J, Covas MI, Farre M, Pujadas M, Fito M, Khymenets O, Andres-Lacueva C, Roset P, Joglar J, Lamuela-Raventós RM, Torre R. Matrix effects on the bioavailability of resveratrol in humans. Food Chem. 2010;120(4):1123-1130. doi:10.1016/j.foodchem.2009.11.032.

104. Tomé-carneiro J, Larrosa M, González-sarrías A, Tomás-barberán FA, Teresa M, Espín JC. Resveratrol and Clinical Trials: The Crossroad from In Vitro Studies to Human Evidence. Curr Pharm Des. 2013:6064-6093.

105. Zalba S, Hagen TLM. Cell membrane modulation as adjuvant in cancer therapy. Cancer Treat Rev. 2017;52:48-57. doi:10.1016/j.ctrv.2016.10.008.

106. Neves AR, Lucio M, Lima JL, Reis S. Resveratrol in Medicinal Chemistry: A Critical Review of Its Pharmacokinetics, Drug-Delivery, and Membrane Interactions. Curr Med Chem. 2014. doi:10.2174/092986712799945085.

107. Hsieh TC. Uptake of resveratrol and role of resveratrol-targeting protein, quinone reductase 2 , in normally cultured human prostate cells. Asian J Androl. 2009;(6):653-661. doi:10.1038/aja.2009.56.

108. Lin HY, Lansing L, Merillon J, Davis FB, Tang H, Shih A, Vitrac X, Krisa S, Keating T, Cao HJ, Bergh J, Quackenbush, Davis PJ. Integrin $\alpha \mathrm{V} \beta 3$ contains a receptor site for resveratrol. FJ Express. 2006;(May 2014). doi:10.1096/fj.06-5743fje.

109. Varoni EM, Lo Faro AF, Sharifi-Rad J, Iriti M. Anticancer Molecular Mechanisms of 
Resveratrol. Front Nutr. 2016;3:8. doi:10.3389/fnut.2016.00008.

110. Singh SK, Jr JWL, Singh R. Reversal of drug resistance by planetary ball milled (PBM) nanoparticle loaded with resveratrol and docetaxel in prostate cancer. Cancer Lett. 2018;427:49-62. doi:10.1016/j.canlet.2018.04.017.

111. Pan M. Antiobesity molecular mechanisms of action: Resveratrol and pterostilbene. Biofactors. 2018;50-60. doi:10.1002/biof.1409.

112. Frazzi R and Guardi M .Cells L. Cellular and Molecular Targets of Resveratrol on Lymphoma and Leukemia Cells. Molecules. 2017:1-15. doi:10.3390/molecules22060885.

113. Patel KR, Brown VA, Jones DJL, Britton RG, Hemingway D, Miller AS, West KP, Booth TD, Perloff M, Crowell JA, Brenner DE, Steward WP, Gescher AJ, Brown K. Clinical pharmacology of resveratrol and its metabolites in colorectal cancer patients. Cancer Res. 2011;70(19):7392-7399. doi:10.1158/0008-5472.CAN-10-2027.

114. Patel KR, Andreadi C, Britton RG, Horner-Glister E, Karmokar A, Sale S, Brown VA, Brenner DE, Singh R, Steward WP, Gescher AJ, Brown K. Sulfate metabolites provide an intracellular pool for resveratrol generation and induce autophagy with senescence. Sci Transl Med. 2013;5(205):205ra133. doi: 10.1126/scitranslmed.3005870.

115. Salehi B, Mishra AP, Nigam M, Sener B, Kilic M. Sharifi-Rad M, Fokou PVT, Martins N, Sharifi-Rad J. Resveratrol: A Double-Edged Sword in Health Benefits. Biomedicines. 2018:1-20. doi:10.3390/biomedicines6030091.

116. Radkar V, Hardej D, Lau-cam C, Billack B. Evaluation of Resveratrol and Piceatannol 
Cytotoxicity in Macrophages, T Cells, and Skin Cells. Arh Hig Rada Toksikol. 2007;58:293-304. doi:10.2478/v10004-007-0020-8.

117. Lecomte S, Demay F, Ferri F, Pakdel F. Phytochemicals Targeting Estrogen Receptors : Beneficial Rather Than Adverse Effects? Int. J. Mol. Sci. 2017:1-19. doi:10.3390/ijms18071381.

118. Chakraborty S, Levenson AS, Biswas PK. Structural insights into Resveratrol's antagonist and partial agonist actions on estrogen receptor alpha. BMC Struct Biol. 2013;13(1):1. doi:10.1186/1472-6807-13-27.

119. Nwachukwu JC, Srinivasan S, Bruno NE, Parent AA, Hughes TS, Pollock JA, Gjyshi O, Cavett V, Nowak J, Garcia-Ordonez RD, Houtman R, Griffin PR, Kojetin DJ, Katzenellenbogen JA, Conkright MD, Nettles KW. Resveratrol modulates the inflammatory response via an estrogen receptor-signal integration network. Elife. 2014;3:e02057. doi:10.7554/eLife.02057.

120. Elshaer M, Chen Y, Wang XJ, Tang X. Resveratrol: An overview of its anti-cancer mechanisms. Life Sci. 2018;207:340-349. doi:10.1016/j.lfs.2018.06.028.

121. Uberti F, Morsanuto V, Aprile S, Ghirlanda S, Stoppa I, Cochis A, Grosa G, Rimondini L, Molinari C. Biological effects of combined resveratrol and vitamin D3 on ovarian tissue. Research. 2017:1-14. doi:10.1186/s13048-017-0357-9.

122. Cipolletti M, Fernandez VS, Montalesi E, Marino M, Fiocchetti M. Beyond the Antioxidant Activity of Dietary Polyphenols in Cancer: the Modulation of Estrogen 
Receptors ( ERs ) Signaling. Int. J. Mol. Sci. 2018;19(9):2624. doi:10.3390/ijms19092624.

123. Walle T, Hsieh F, DeLegge MH, Oatis JE, Walle UK. High absorption but very low bioavailability of oral reseveratrol in humans. Drug Metab Dispos. 2004;32(12):13771382. doi:10.1124/dmd.104.000885.

124. Dandawate PR, Subramaniam D, Jensen RA, Anant S. Targeting cancer stem cells and signaling pathways by phytochemicals: Novel approach for breast cancer therapy. Semin Cancer Biol. 2016:192-208. doi:10.1016/j.semcancer.2016.09.001.

125. Scarlatti F, Sala G, Somenzi G, Signorelli P, Sacchi N, Ghidoni R. Resveratrol induces growth inhibition and apoptosis in metastatic breast cancer cells via de novo ceramide signaling. F.J. Express. 2003;. doi:10.1096/fj.03-0292fje.

126. Singh SK, Banerjee S, Acosta EP, Lillard JW, Singh R. Resveratrol induces cell cycle arrest and apoptosis with docetaxel in prostate cancer cells via a p53 / p21 WAF1 / CIP1 and p27 KIP1 pathway. Oncotarget. 2017;8(10):17216-17228.

127. Hahnvajanawong C, Boonyanugomol W, Pattanapanyasat K, Sripa B, Namwat N, Pinmai $\mathrm{K}$, Tassaneeyakul W, Reutrakul V. Inhibition of cell cycle progression and apoptotic activity of resveratrol in human intrahepatic cholangiocarcinoma cell lines. Asian Biomed. 2011;5(6):775-786. doi:10.5372/1905-7415.0506.116.

128. Rauf A, Imran M, Butt MS, Nadeem M, Peters DG, Mubarak MS. Resveratrol as an anticancer agent: A review. Crit Rev Food Sci Nutr. 2018;58(9):1428-1447. doi:10.1080/10408398.2016.1263597. 
129. Ciolino HP, Daschner PJ, Yeh GC. Resveratrol inhibits transcription of CYP1A1 in vitro by preventing activation of the aryl hydrocarbon receptor. Cancer Res. 1998;58(24):570712.

130. Shamsara J, Shahir-sadr A. Developing a CoMSIA Model for Inhibition of COX-2 by Resveratrol Derivatives. Iran J Pharm Res. 2016;15:459-469.

131. Gong W, Zhao N, Zhang Z, Zhang Y, Yan L, Li J. The inhibitory effect of resveratrol on COX-2 expression in human colorectal cancer: a promising therapeutic strategy. Eur Rev Med Pharmacol Sci. 2017:1136-1143.

132. Zykova TA, Zhu F, Zhai X, Ma W, Ermakova SP, Lee KW, Bode AM, Dong Z. Resveratrol directly targets COX- 2 to inhibit carcinogenesis. Mol Carcinog. 2008;47(10):797-805. doi:10.1002/mc.20437.

133. Baur JA, Pearson KJ, Price NL, Jamieson HA, Lerin C, Kalra A, Prabhu VV, Allard JS, Lopez-Lluch G, Lewis K, Pistell PJ, Poosala S, Becker KG, Boss O, Gwinn D, Wang M, Ramaswamy S, Fishbein KW, Spencer RG, Lakatta EG, Le Couteur D, Shaw RJ, Navas P, Puigserver P, Ingram DK, de Cabo R, Sinclair DA. Resveratrol improves health and survival of mice on a high-calorie diet. Nature. 2006;444:337.

134. Bhullar KS, Hubbard BP. Lifespan and healthspan extension by resveratrol. Biochim Biophys Acta - Mol Basis Dis. 2015;1852(6):1209-1218. doi:https://doi.org/10.1016/j.bbadis.2015.01.012.

135. Kovacic P, Somanathan R. Multifaceted approach to resveratrol bioactivity Focus on 
antioxidant action, cell signaling and safety. Oxid Med Cell Longev. 2010;3(2):86-100. doi: 10.4161/oxim.3.2.11147.

136. McBride WJ, D'Souza CA, Karacay H, Sharkey RM, Goldenberg DM. New lyophilized kit for rapid radiofluorination of peptides. Bioconjug Chem. 2012;23(3):538-547. doi:10.1021/bc200608e.A.

137. Singh CK, George J, Ahmad N. Resveratrol-based combinatorial strategies for cancer management. Ann N Y Acad Sci. 2013;1290(1):113-121. doi:10.1111/nyas.12160.

138. Morris VL, Toseef T, Nazumudeen FB, Rivoira C, Spatafora C, Tringali C, Rotenberg SA. Anti-tumor properties of cis-resveratrol methylated analogs in metastatic mouse melanoma cells. Mol Cell Biochem. 2015;402(0):83-91. doi:10.1007/s11010-014-2316-8.

139. Zhao H, Chen S, Gao K, Zhou Z, Wang C, Shen Z, Guo Y, Li Z, Wan Z, Liu C, Mei X. Resveratrol protects against spinal cord injury by activating autophagy and inhibiting apoptosis mediated by the SIRT1/AMPK signaling pathway. Neuroscience. 2017;348:241-251. doi:10.1016/j.neuroscience.2017.02.027.

140. Bhat K.P.L, Pezzuto J.M. Resveratrol Exhibits Cytostatic and Antiestrogenic Properties with Human Endometrial Adenocarcinoma (Ishikawa) Cells. Cancer Research. $2001 ; 6137-6144$

141. Han YS, Bastianetto S, Dumont Y, Quirion R. Specific Plasma Membrane Binding Sites for Polyphenols, Including Resveratrol, in the Rat Brain. J. Pharmacol. Exp. Ther. 2006; $238-245$ 
142. Mehta J, Rayalam S, Wang X. Cytoprotective Effects of Natural Compounds against Oxidative Stress. Antioxidants (Basel). 2018:1-20. doi:10.3390/antiox7100147.

143. Yahfoufi N, Alsadi N, Jambi M, Matar C. The Immunomodulatory and AntiInflammatory Role. Nutrients. 2018:1-23. doi:10.3390/nu10111618.

144. Kuršvietienė L, Stanevičienė I, Mongirdienė A, Bernatonienė J. Multiplicity of effects and health benefits of resveratrol. Medicina (Kaunas). 2016;2:3-10. doi:10.1016/j.medici.2016.03.003.

145. Williams P, Sorribas A, Howes M-JR. Natural products as a source of Alzheimer's drug leads. Nat Prod Rep. 2011;28(1):48-77. doi:10.1039/c0np00027b.

146. Kumar S, Eroglu E, Stokes JA 3rd, Scissum-Gunn K, Saldanha SN, Singh UP, Manne U, Ponnazhagan S, Mishra MK. Resveratrol induces mitochondria-mediated, caspaseindependent apoptosis in murine prostate cancer cells. Oncotarget. 2017;8(13):2089520908. www.impactjournals.com/oncotarget.

147. Brabletz T, Kalluri R, Nieto MA, Weinberg RA. EMT in cancer. Nat Rev Cancer. 2018;18:128. doi: 10.1038/nrc.2017.118.

148. Park SJ, Ahmad F, Um JH, Brown AL, Xu X, Kang H, Ke H, Feng X, Ryall J, Philp A, Schenk S, Kim MK, Sartorelli V, Chung JH. Specific Sirt1 Activator-mediated Improvement in Glucose Homeostasis Requires Sirt1-Independent Activation of AMPK. EBioMedicine. 2017;18:128-138. doi:https://doi.org/10.1016/j.ebiom.2017.03.019.

149. Dai H, Sinclair DA, Ellis JL, Steegborn C. Pharmacology \& Therapeutics Sirtuin 
activators and inhibitors: Promises, achievements, and challenges. Pharmacol Ther. 2018;188:140-154. doi:10.1016/j.pharmthera.2018.03.004.

150. Treviño-Saldaña N, García-Rivas G. Regulation of Sirtuin-Mediated Protein Deacetylation by Cardioprotective Phytochemicals. Oxid Med Cell Longev. 2017;1750306. doi:10.1155/2017/1750306.

151. Howitz KT, Bitterman KJ, Cohen HY, Lamming DW, Lavu S, Wood JG, Zipkin RE, Chung P, Kisielewski A, Zhang L, Scherer B, Sinclair DA. Small molecule activators of sirtuins extend Saccharomyces cerevisiae lifespan. Nature. 2003;425:191. https://doi.org/10.1038/nature01960.

152. Gertz M, Nguyen GTT, Fischer F, Suenkel B, Schlicker C, Franzel B, Tomaschewski J, Aladini F, Becker C, Wolters D, Steegborn C. A Molecular Mechanism for Direct Sirtuin Activation by Resveratrol. PLoS One. 2012;7(11):e49761. https://doi.org/10.1371/journal.pone.0049761.

153. Kasiotis KM, Pratsinis H, Kletsas D, Haroutounian SA. Resveratrol and related stilbenes: Their anti-aging and anti-angiogenic properties. Food Chem Toxicol. 2013;61:112-120. doi:https://doi.org/10.1016/j.fct.2013.03.038.

154. Heo JR, Kim SM, Hwang KA, Kang JH, Choi KC. Resveratrol induced reactive oxygen species and endoplasmic reticulum stress - mediated apoptosis, and cell cycle arrest in the A375SM malignant melanoma cell line. Int J Mol Med. 2018:1427-1435. doi:10.3892/ijmm.2018.3732. 
155. Truong V, Jun M, Jeong W. Role of resveratrol in regulation of cellular defense systems against oxidative stress. BioFactors. 2018;36-49. doi:10.1002/biof.1399.

156. Ko J, Sethi G, Um J, Shanmugam MK, Arfuso F, Kumar AP, Bishayee A, Ahn KS. The Role of Resveratrol in Cancer Therapy. Int. J. Mol. Sci. 2017;18(12):2589. doi:10.3390/ijms18122589.

157. Mirossay L, Varinsk L, Mojžiš J. Antiangiogenic Effect of Flavonoids and Chalcones : An Update. Int J Mol Sci. 2018. doi:10.3390/ijms19010027.

158. Bishayee A. Cancer Prevention and Treatment with Resveratrol: From Rodent Studies to Clinical Trials. Cancer Prev Res. 2009;2(5):409-418. doi:10.1158/1940-6207.

159. Filippi-chiela EC, Villodre ES, Zamin LL, Lenz G. Autophagy Interplay with Apoptosis and Cell Cycle Regulation in the Growth Inhibiting Effect of Resveratrol in Glioma Cells. PLOS One. 2011;6(6). doi:10.1371/journal.pone.0020849.

160. Jin Z, Feng WEI, Ji Y, Jin L. Resveratrol mediates cell cycle arrest and cell death in human esophageal squamous cell carcinoma by directly targeting the EGFR signaling pathway. Oncol. Lett. 2017:347-355. doi:10.3892/ol.2016.5391.

161. Walle T, Hsieh F, Delegge MH, Oatis JE, Walle UK. High absortion but very low bioavaibility of oral resveratrol in humans. Drug Metab isposition. 2004;32(12):13771382. doi:10.1124/dmd.104.000885.

162. Godin B, Touitou E. Mechanism of bacitracin permeation enhancement through the skin and cellular membranes from an ethosomal carrier. J Control Release. 2004;94(2):365- 
379. doi:https://doi.org/10.1016/j.jconrel.2003.10.014.

163. Machida Y, Onishi H, Kurita A, Hata H, Morikawa A, Machida Y. Pharmacokinetics of prolonged-release CPT-11-loaded microspheres in rats. $J$ Control Release. 2000;66(2):159-175. doi:https://doi.org/10.1016/S0168-3659(99)00267-9.

164. Şanlı O, Karaca İ, Işıklan N. Preparation, characterization, and salicylic acid release behavior of chitosan/poly(vinyl alcohol) blend microspheres. J Appl Polym Sci. 2009;111(6):2731-2740. doi:10.1002/app.29319.

165. Chao P, Deshmukh M, Kutscher HL, Gao D, Rajan SS, Hu P, Laskin DL, Stein S, Sinko PJ. Pulmonary targeting microparticulate camptothecin delivery system: anticancer evaluation in a rat orthotopic lung cancer model. Anticancer Drugs. 2010;21(1):65-76. doi:10.1097/CAD.0b013e328332a322.

166. Gavini E, Alamanni MC, Cossu M, Giunchedi P. Tabletted microspheres containing Cynara scolymus (var. Spinoso sardo) extract for the preparation of controlled release $\begin{array}{llll}\text { nutraceutical } & \text { matrices. } & J & \text { Microencapsul. 2005;22(5):487-499. }\end{array}$ doi:10.1080/02652040500099919.

167. Bhasin B, Londhe VY. An overview of transfersomal drug delivery. IJPSR. 2018;9(6):2175-2184. doi:10.13040/JJPSR.0975-8232.9(6).2175-84.

168. Obeid MA, Al Qaraghuli MM, Alsaadi M, Alzahrani AR, Niwasabutra K, Ferro VA. Delivering natural products and biotherapeutics to improve drug efficacy. Ther Deliv. 2017;8(11):947-956. doi:10.4155/tde-2017-0060. 
169. Din F, Aman W, Ullah I, Qureshi OS, Mustapha O, Shafique S, Zeb A. Effective use of nanocarriers as drug delivery systems for the treatment of selected tumors. Int $J$ Nanomedicine. 2017:7291-7309. doi: 10.2147/IJN.S146315.

170. Leso V, Iavicoli I. Palladium Nanoparticles: Toxicological Effects and Potential Implications for Occupational Risk Assessment. Int. J. Mol. Sci. 2018;19(2):503. doi:10.3390/ijms19020503.

171. Chauhan S. Use of cellulose and its derivatives for metal ion sorption. J Chem Pharm Res. 2010;2(4):602-611.

172. Gurunathan S, Kim E, Han JW, Park JH, Kim J. Green Chemistry Approach for Synthesis of Effective Anticancer Palladium Nanoparticles. Molecules. 2015;20(12):22476-22498. doi:10.3390/molecules201219860.

173. Lu Y, Li W, Hu B, Yu S. Size-controllable palladium nanoparticles immobilized on carbon nanospheres for nitroaromatic hydrogenation. J. Mater. Chem. A. 2013;1:37833788. doi:10.1039/C3TA00159H.

174. Qin Y, Kong Y, Tao Y, Li S. In situ synthesis of highly loaded and ultrafine Pd nanoparticles-decorated graphene oxide for glucose biosensor application. J. Mater. Chem. 2012;(November). doi:10.1039/C2JM35321K.

175. Gananathan P, Rao AP, Ganesan S, Manickan E. Review of Laser in Nanophotonics - A Literature Study for Cellular Mechanism. Integr Cancer Biol Res. 2017;5:1-14.

176. Melo-diogo D De, Pais-silva C, Dias DR, Moreira AF, Correia IJ. Strategies to Improve 
Cancer Photothermal Therapy Mediated by Nanomaterials. Adv Healthc Mater. 2017. doi:10.1002/adhm.201700073.

177. Kruger CA, Abrahamse H. Utilisation of Targeted Nanoparticle Photosensitiser Drug Delivery Systems for the Enhancement of Photodynamic Therapy. Molecules. 2018. doi:10.3390/molecules23102628.

178. Lee SS. Targeted nanotherapy for prostate cancer: Applications for cancer therapeutics and imaging. 2017.

179. Rubio-ruiz B, Pérez-lópez AM, Bray TL, Lee M, Serrels A, Prieto M, Arruebo M, Carragher NO, Sebastián V, Unciti-Broceta A. High-Precision Photothermal Ablation using Biocompatible Palladium Nanoparticles and Laser Scanning Microscopy. ACS Appl Mater Interfaces. 2018;10(4):1-16

180. Bray TL, Lee M, Serrels A, Arruebo M, Carragher NO. High-Precision Photothermal Ablation Using Biocompatible Palladium Nanoparticles and Laser Scanning Microscopy. ACS Appl Mater Interfaces. 2018. doi:10.1021/acsami.7b17282.

181. Kim H, Beack S, Han S, Shin M, Lee T, Park Y, Kim KS, Yetisen AK, Yun SH, Kwon W, Hahn SK. Multifunctional Photonic Nanomaterials for Diagnostic, Therapeutic , and Theranostic Applications. Adv. Mater. 2018;1701460:1-33. doi:10.1002/adma.201701460.

182. Yan G, Li A, Zhang A, Sun Y, Liu J. Polymer-Based Nanocarriers for Co-Delivery and Combination of Diverse Therapies against Cancers. Nanomaterials. 2018. doi:10.3390/nano8020085. 
183. Liu Y, Bhattarai P, Dai Z, Chen X. Photothermal therapy and photoacoustic imaging via nanotheranostics in fighting cancer. Chem Soc Rev. 2019. doi:10.1039/C8CS00618K.

184. Miller MA, Askevold B, Mikula H, Kohler RH, Pirovich D, Weissleder R. chemistry. Nat Commun. 2017;8:1-13. doi:10.1038/ncomms15906.

185. Reale M, Vianale G, Lotti LV, Mariani-Costantini R, Perconti S, Cristaudo A, Leopold K, Antonucci A, Di Giampaolo L, Iavicoli I, Di Gioacchino M, Boscolo P. Effects of palladium nanoparticles on the cytokine release from peripheral blood mononuclear cells of palladium-sensitized women. J Occup Environ Med. 2011;53(9):1054-1060. doi:10.1097/JOM.0b013e318228115e.

186. Iavicoli I, Fontana L, Leso V, Corbi M, Marinaccio A, Leopold K, Schindl R, Lucchetti D, Calapà F, Sgambato A. Subchronic exposure to palladium nanoparticles affects serum levels of cytokines in female Wistar rats. Hum Exp Toxicol. 2017;37(3):309-320. doi:10.1177/0960327117702952.

187. Battke F, Leopold K, Maier M, Schmidhalter U, Schuster M. Palladium exposure of barley: uptake and effects. Plant Biol. 2008;10(2):272-276. doi:10.1111/j.14388677.2007.00017.x.

188. Hildebrand H, Kühnel D, Potthoff A, Mackenzie K, Springer A, Schirmer K. Evaluating the cytotoxicity of palladium / magnetite nano-catalysts intended for wastewater treatment. Environ Pollut. 2010;158:65-73. doi:10.1016/j.envpol.2009.08.021.

189. Leso V, Fontana L, Marinaccio A, Leopold K, Fanali C, Lucchetti D, Sgambato A, 
Iavicoli I. Palladium nanoparticle effects on endocrine reproductive system of female rats.

Hum Exp Toxicol. 2018;37(10):1069-1079. doi:10.1177/0960327118756722. 


\section{CHAPTER 2}

\section{GREEN NANOTECHNOLOGY}

Green nanotechnology is development of ecofriendly nanomaterials using plant bioactive compounds (phytochemicals, carbohydrates, etc.) rather than toxic chemicals (e.g., sodium borohydride, hydrazine, sodium citrate, etc.) to minimize side effects on human health, toxic waste and potential environmental risks. The utilization of green chemistry in the synthesis of nanoparticles have endowed them with biocompatibility, environmentally healthy, and stability in physiological solutions that mimic in vivo conditions, as well as facilitating further modification for specific-targeting therapies. ${ }^{1-4}$ Nanotechnologies bring about a paradigm advantage in packaging, storage, delivery and enhancing the bioavailability of active phytochemicals from various plants and herbal sources. Professor Kattesh Katti's laboratory has pioneered the application of green nanotechnology for the development of nanoparticles of gold, silver, palladium and a host of other metallic precursors. ${ }^{5-8}$ That is, abiding by the $\underline{12 \text { design }}$ principles of green chemistry outlined by Anastas and Warner within the American Chemical Society. ${ }^{9}$

\subsection{Green nanotechnology in cancer therapy - combinational modality}

An efficient strategy for overcoming metastasis is through combinational therapy that makes use of a natural cancer chemopreventive agent as an adjuvant treatment to complement existing therapy. The use of green nanotechnology provides an alternative solution that can surpass current obstacles for cancer treatments, ${ }^{4,10,11}$ thereby, attaining the maximum therapeutic efficacy against tumor cells with the minimum concentration of the nanodrug. ${ }^{12}$ Green nanotechnology 
allows the development of nanoparticles through direct interaction of electron-rich anti-oxidants of plant origin. ${ }^{13,14,21,20}$ Gurunathan et al. ${ }^{15}$ demonstrated the use of leaf extract of Evolvulus alsinoides for the synthesis of palladium nanoparticles and reported then to be in vitro efficacy against A2780 human ovarian cancer cells. Moreover, the results revealed that the anticancer mechanism of the synthesized particles was through increased leakage of lactate dehydrogenase (LDH), elevated levels of reactive oxygen species (ROS), induced autophagy and autophagic cell death confirmed by transmission electron microscopy (TEM). The loss of mitochondrial membrane potential (MMP) and enhanced caspase-3 activity followed by DNA fragmentation accessed using terminal deoxynucleotidyl transferase (TdT) dUTP Nick-End Labeling (TUNEL) assay was attributed to PdNPs. ${ }^{15}$ There are currently ongoing efforts on the development of metallic nanoparticles as antineoplastic agents for a myriad of cancers. It is apparent from the above mentioned data that both the metal and the ligand determine the overall biological activity of the nanoformulations. The principle of suitable cancer therapy is not based on a single therapeutic intervention but on the pivotal philosophy of restoring homeostasis. ${ }^{16}$

\subsection{Green synthesis of palladium nanoparticles (PdNPs)}

The synthesis of palladium nanoparticles has been previously reported using various plants extracts (black pepper, tea, soybean, seaweed and other plant materials). ${ }^{4,7-10,13,14,17-31}$ The use of a single bioactive compound from the extracts facilitates the reproducibility of the synthesized nanoparticles of the same size and shape optimally suited to penetrate tumor vasculature associated with leaky blood vessels, which are manifested in all human tumors. ${ }^{32}$ This can be capitalized by synthesizing nanoparticles that are conjugated/encapsulated by natural anticancer agents as a combinational therapeutic strategy. 
Resveratrol (Res) has three hydroxyl groups that can serve as electron donors to transform palladium metal salts into zero-valent palladium nanoparticles. ${ }^{4}$ Moreover, in the case of hydrogen bonds formed in phenoxy radicals, phenoxy radical oxygen can act as a proton acceptor in the presence of substituents that can serve as proton donors. ${ }^{33}$ The flavonoid B-ring of Res is more important than A-ring from a bond dissociation enthalpy (BDE) - represents the ease of hydrogen donation of phytochemicals; this is because the radical structure formed after $\mathrm{H}$-atom abstraction from the 4'-OH group can be stabilized by the resonance between the two rings for strong electron-donating groups. ${ }^{34,35}$ The nucleophilic and electrophilic sites in Res are expressed in term of different color codes as shown in Figure $12 .^{33,34,34}$

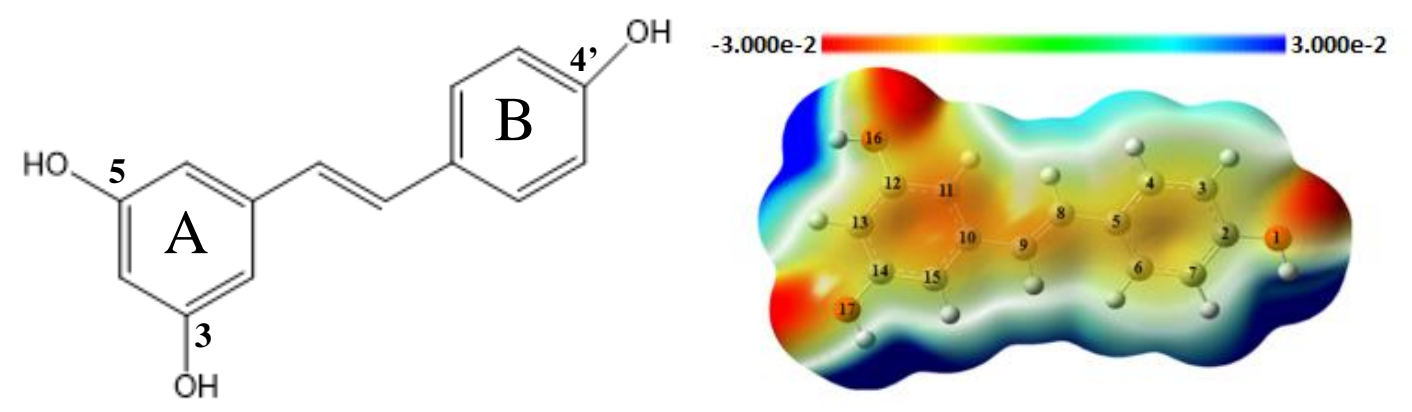

Figure 12: Resveratrol A- and B-ring, and its molecular electrostatic potential (MEP), where the deep red color indicates an electron-rich site, whereas deep blue indicates an electron-deficient site.

The electrostatic potential of a molecule indicates charge distribution of the reactive site of a molecule as an indicator of ligand binding. Moreover, the MEP reflects the dominant role of $\mathrm{OH}$ groups, with the negative (red) and positive (blue) potentials. The density functional theory (DFT) calculates BDE to determine the measure of the strength of a chemical bond $\mathrm{O}-\mathrm{H}$. The 4$\mathrm{OH}$ of Res has a BDE of $325.7 \mathrm{~kJ} \mathrm{~mol}^{-1}$, while $3-\mathrm{OH}$ and $5-\mathrm{OH}$ are 348.2 and $353.6 \mathrm{~kJ} \mathrm{~mol}^{-1}$, 
respectively. ${ }^{35,36}$ The hydroxyl group with a lowest $\mathrm{BDE}$ is more reactive; therefore, 4-OH group has highly condensed electron density area localized over the oxygen atoms with electron lone pairs along with the ionization potential (IP) and electronegativity of the resveratrol play an important role in chemical reactivity behavior. ${ }^{36}$ The IP of Res is $155.68 \mathrm{kcal} \mathrm{mol}^{-1}$ representing the ease of electron donation with a highest occupied molecular orbital (HOMO) energy at -7.5 $\mathrm{eV}$ and lowest unoccupied molecular orbital (LUMO) at $2.53 \mathrm{eV} . .^{34,37}$

The large surface-to-area ratio and extraordinary surface kinetics of atoms that make up the nanoparticles (gold, palladium, iron oxide and various nanoparticles) allow highly effective loading of anticancer phytochemical compounds in their nascent form onto the surface of nanoparticles. ${ }^{38}$ The strong propensity of phytochemical-conjugated nanoparticles (size range: 15-30 nm) to internalize into tumor cells, through a combination of pathways (e.g., endocytosis and phagocytic). ${ }^{10}$ This allows for efficient transport and delivery of a reservoir of phytochemicals conjugated to the nanoparticles into the cellular matrix. Several studies have shown that Res exerts antimicrobial and antioxidant activities, which extend to anti-tumor properties, inhibitory effects on type II $5 \alpha$-reductase (prostate-specific, membrane-associated enzyme that catalyzes the conversion of testosterone to dihydrotestosterone) in vitro, and in vivo growth-inhibitory activity against ascitic fibrosarcoma. ${ }^{21,22,39}$ Thereby, Res can be a competitive inhibitor of the type II steroid $5 \alpha$-reductase used to treat benign prostatic hyperplasia.

Res has been reported to suppress M2-like polarization of tumor-associated macrophages (TAMs) which is associated with pro-tumoral features, this subsequently inhibited tumor growth in a mouse lung cancer xenograft model. ${ }^{40-43}$ Leischner et al. ${ }^{42}$ reviewed Res to be a promising natural stimulant of immune responses including $\mathrm{NK}$ cells, CD8+ and CD4+ T-cells. 
Mechanistic investigations revealed that Res act as a natural inhibitor for the matrix metalloproteinase (MMP)-7 and -9 which play a role in malignant tumor invasion, and tumor metastasis. $^{40,44}$ There is conclusive evidence that the mechanism of modulation of MMP-7 and -9 is due to the innate ability of Res to inhibit $\beta$-catenin pathway and decreased activity of signal transducer and activator of transcription 3 (STAT3). ${ }^{45,46}$ Gum Arabic (GA) a complex mixture of polysaccharides (galactose backbone branched with mannose, arabinose, rhamnose, $\alpha$ - and $\beta$ sugar residues) and glycoproteins that are cross-linked forming arabinogalactan proteins, which makes up the majority of its overall structure. ${ }^{47}$

The exudates of Acacia seyal trees are the source of GA and it has been used as a stabilizer in the food industry. ${ }^{48-51}$ Therefore, creation of palladium nanoparticles encapsulated with anti-tumor Res and GA to optimize stability presents an unprecedented opportunity to exploit the dual therapy effects of both the palladium nanoparticles and also the surface coated Res. ${ }^{52}$ There is also evidence that surface encapsulation of Res on nanoparticles surface would enhance their bioavailability and facilitate synergistic biorthogonal response in vivo. ${ }^{53}$ This can also be extended on how resveratrol-derived phenol nanoparticles can be internalized into the laminin receptor positive cancerous cells. Res can be used to sensitize tumor cells through an additive, potentiation and synergistic effects with PdNPs for a augmented therapeutic response that creates an optimal modality approach, which can ultimately facilitate clinical applications of combination regimens in the future of cancer therapy.

\subsection{Combinational treatment parameter}

It is apparent that monotherapeutic options for cancer treatment may not be able to exhibit the most beneficial effects, thereby a combinational treatment approach that target tumor specific 
receptors may result in a greater therapeutic efficiency. Chiou et al. ${ }^{54}$ alluded that there are crucial principles that need to be considered when developing a combinational treatment. That is, neutralizing responses and overlapping unacceptable toxicity that is greater than clinical benefit. The use of natural product-based agents such as Res in combination with nanodrugs is a promising approach. Therefore, the above mentioned experimental rationale and the synergistic therapeutic effects of Res-derived phenols encapsulated PdNPs as drug-like metal architecture prompted us to hypothesize that the production of nanoparticles of palladium encapsulated with Res would create the next generation of cancer therapy nanomedicine agents capable of delivering potent doses of both resveratrol-derived phenols and polyphenols encapsulating the nanoparticles directly to tumor cells. Tumor selective affinity of resveratrol on the Pd surface would lead to endocytosis of PdNPs thus providing an additional armamentarium for therapy through NIR $808 \mathrm{~nm}$ laser induced heat production around PdNPs present within tumor cells providing a synergistic biorthogonal response. We further hypothesized that the mechanism of cell death will involve immunomodulatory characteristics because resveratrol has been known to induce immunomodulation by their ability to target cell signaling pathways as well as macrophages. The amalgamations of resveratrol conjugated palladium nanopharmaceutical could be a new addition to the nanomedical repertoire in the near future. 


\subsection{Aim and objectives of the study}

\subsubsection{Aim}

The main goal of this research was to explore the production of biocompatible palladium nanoparticles using resveratrol to treat, image and evaluate the efficacy of the formulation in prostate cancer.

\subsubsection{Objectives}

This work was classified into three parts with the following objectives:

1. The production and characterization of palladium nanoparticles synthesized using resveratrol (Res-PdNPs) for the imaging and treatment of prostate cancer.

2. To increase the resveratrol-derived phenols and polyphenols corona loading on the palladium nanoparticle surface capable of providing adjuvant therapeutic benefits through delivering potent doses of both resveratrol and nanoparticles directly to tumor cells.

3. The prostate tumor selective affinity of Res-PdNPs via endocytosis was evaluated.

Our overarching goal for this study is to exploit the inherent anti-angiogenesis and pro-apoptotic properties of palladium nanoparticles through synergistic anti-tumor characteristics of resveratrol-all aimed at developing a new class green nanotechnology-based phytochemical embedded palladium nanoparticles for applications in prostate cancer therapy. 


\section{REFERENCES}

1. Katti K, Chanda N, Shukla R, Zambre A, Suibramanian T, Kulkarni RR. Kannan R, Katti KV. Green Nanotechnology from Cumin Phytochemicals: Generation of Biocompatible Gold Nanoparticles. Int J Green Nanotechnol Biomed. 2009;39-52. doi:10.1080/19430850902931599.

2. Parveen K, Banse V, Ledwani L. Green synthesis of nanoparticles: Their advantages and disadvantages. AIP Conf Proc. 2016;1724. doi:10.1063/1.4945168.

3. Kalpana VN, Rajeswari VD. A Review on Green Synthesis, Biomedical Applications , and Toxicity Studies of ZnO NPs. Bioinorg Chem Appl. 2018;1-12. doi:10.1155/2018/3569758.

4. Geraldes AN, Alves A, Leal J, Estrada-Villegas, Lincopan N, Katti KV, Lugao AB. Green Nanotechnology from Plant Extracts: Synthesis and Characterization of Gold Nanoparticles. ANP. 2016;5:176-185.

5. Katti K V. Renaissance of nuclear medicine through green nanotechnology: functionalized radioactive gold nanoparticles in cancer therapy - my journey from chemistry to saving human lives. J Radioanal Nucl Chem. 2016;309(1):5-14. doi:10.1007/s10967-016-4888-0.

6. Chanda N, Shukla R, Zambre A, Mekapothula S, Kulkarni RR, Katti K, Bhattacharyya K, Fent GM, Casteel SW, Boote EJ, Viator JA, Upendran A, Kannan R, Katti KV. An Effective Strategy for the Synthesis of Biocompatible Gold Nanoparticles Using Cinnamon Phytochemicals for Phantom CT Imaging and Photoacoustic Detection of Cancerous Cells. Pharm Res. 2011;28(2):279-291. doi:10.1007/s11095-010-0276-6. 
7. Katti KK, Kattumuri V, Bhaskaran S, Katti K V, Kannan R. Facile and General Method for Synthesis of Sugar-Coated Gold Nanoparticles. Int J Green Nanotechnol Biomed. 2009;1(1):B53-B59. doi:10.1080/19430850902983848

8. Shukla R, Nune SK, Chanda N, Katti K, Mekapothula S, Kulkarni RR, Welshons WV, Kannan R, Katti KV. Soybeans as a phytochemical reservoir for the production and stabilization of biocompatible gold nanoparticles. Small. 2008;4(9):1425-1436. doi:10.1002/smll.200800525.

9. Anastas, P.T.; Warner, J. C. Green Chemistry: Theory and Practice, Oxford University Press: New York, 1998, p.30.

10. Khoobchandani M, Zambre A, Katti K, Lin C, Katti K V. Green Nanotechnology from Brassicaceae: Development of Broccoli Phytochemicals - Encapsulated Gold Nanoparticles and Their Applications in Nanomedicine. Int J Green Nanotechnol Biomed. 2013. doi:10.1177/1943089213509474.

11. Axiak-Bechtel S, Upendran A, Lattimer J, Kelsey J, Cutler C, Selting K, Bryan J, Henry C, Boote E, Tate D, Bryan M, Katti KV, Kannan R. Gum arabic-coated radioactive gold nanoparticles cause no short-term local or systemic toxicity in the clinically relevant canine model of prostate cancer. Int J Nanomedicine. 2014;9(1):5001-5011. doi:10.2147/IJN.S67333.

12. Jahromi EZ. Divsalar A, Saboury AA, Khaleghizadeh S, Mansouri-Torshizi H, Kostova I. Palladium complexes: new candidates for anti-cancer drugs. J Iran Chem Soc. 2018;13(5):967-989. doi:10.1007/s13738-015-0804-8. 
13. Katti K, Chanda N, Shukla R, Zambre A, Suibramanian T, Kulkarni RR, Kannan R, Katti KV. Green Nanotechnology from Cumin Phytochemicals: Generation of Biocompatible Gold Nanoparticles. Int J Green Nanotechnol Biomed. 2009;1(1):B39-B52. doi:10.1080/19430850902931599.

14. Nune SK, Chanda N, Shukla R, Katti K, Kulkarni K, Subramanin T, Mekapothula S, Kannan R, Katti KV. Green nanotechnology from tea: phytochemicals in tea as building blocks for production of biocompatible gold nanoparticles. $J$ Mater Chem. 2009;19(19):2912-2920. doi:10.1039/B822015H.

15. Gurunathan S, Kim E, Han JW, Park JH, Kim J. Green Chemistry Approach for Synthesis of Effective Anticancer Palladium Nanoparticles. Molecules. 2015;20(12):22476-22498. doi:10.3390/molecules201219860.

16. Ramaswamy S. Reflections on current Ayurveda research. J Ayurveda Integr Med. 2018;9:250-251. doi:10.1016/j.jaim.2018.11.001.

17. Chanda N, Kan P, Watkinson LD, Shukla R, Zambre A, Carmack TL, Engelbrecht H, Lever JR, Katti K, Fent GM, Casteel SW, Smith CJ, Miller WH, Jurisson S, Boote E, Robertson JD, Culter C, Dobrovolskaia M, Kannan R, Katti KV. Radioactive gold nanoparticles in cancer therapy: therapeutic efficacy studies of GA-198AuNP nanoconstruct in prostate tumor-bearing mice. Nanomedicine: NBM. 2010;6:201-209, doi:10.1016/j.nano.2009.11.001.

18. Katti KV, Kannan R, Katti K, Nune SK. Stabilized, biocompatible gold nanoparticles and enviro-friendly method for making same. US Patent 8,333,994, 2012. 
19. Kattumuri V, Katti KV, Boote E, Kannan R, Casteel S, Churchill R. Stabilized gold nanoparticle and contrast agent. US Patent 9,549,998, 2017.

20. Katti KV, Kannan R, Cutler CS. Gum arabic coated 198gold radioactive nanoparticles for cancer therapy. US Patent App. 13/293,827, 2012.

21. Kannan R, Katti KK, Katti KV, White HW, Cutler CS. Methods and articles for gold nanoparticle production. US Patent 8,241,393, 2012..

22. Katti KV, Khoobchandani M, Thipe VC, Al-Yasiri AY. Prostate tumor therapy advances in nuclear medicine: green nanotechnology toward the design of tumor specific radioactive gold nanoparticles. J Radioanal Nucl Chem. 2018;318(3):1737-1747. doi:10.1007/s10967-018-6320-4.

23. KV Katti, R Kannan, KK Katti, SK Nune, CS Cutler, Caldwell C, Shukla R, Chanda N, Zambre A, Upendran A. EGCG stabilized gold nanoparticles and method for making same. US Patent 9,358,310, 2016.

24. Chanda N, Kattumuri V, Shukla R, Zambre A, Katti K, Upendran A, Kulkarni RR, Kan P, Fent GM, Casteel SW, Smith CJ, Boote E, Robertson JD, Cutler CS, Lever RR, Katti KV, Kannan R. Bombesin functionalized gold nanoparticles show in vitro and in vivo cancer $\begin{array}{lllll}\text { receptor } & \text { specificity. } & P N A S, & 2010 & 107\end{array}$ https://doi.org/10.1073/pnas.1002143107.

25. Shukla R, Chanda N, Zambre A, Upendran A, Katti K, Kulkarni RR. Laminin receptor specific therapeutic gold efficacy in treating prostate cancer. PNAS, 2012:1-6. 
doi:10.1073/pnas.1121174109.

26. Sinha S, McKnight D, Katti KV, Kannan R, Robertson DJ, Cowden JW, Mohan RR. Gold Nanoparticles Stabilized in Gum Arabic for Corneal Gene Therapy. Invest Ophthalmol Vis Sci. 2008;49(13):4787. http://dx.doi.org/.

27. Mohan RR, Sinha S, Katti K V, Kannan R, Stapleton WM, Schultz GS. Evaluation of Polymeric- and Gold-Nanoparticles for Gene Delivery in the Cornea. Invest Ophthalmol Vis Sci. 2007;48(13):2733. http://dx.doi.org/.

28. Gamal-Eldeen AM, Moustafa D, El-Daly SM, Abo-Zeid MAM, Saleh S, Khoobchandani M, Katti K, Shukla R, Katti KV. Gum Arabic-encapsulated gold nanoparticles for a noninvasive photothermal ablation of lung tumor in mice. Biomed Pharmacother. 2017;89:1045-1054. doi:10.1016/j.biopha.2017.03.006.

29. Chanda N, Shukla R, Katti K V, Kannan R. Gastrin releasing protein receptor specific gold nanorods: breast and prostate tumor avid nanovectors for molecular imaging. Nano Lett. 2009;9(5):1798-805. doi: 10.1021/n18037147.

30. Kannan R, Rahing V, Cutler C, Pandrapragada R, Katti KK, Kattumuri V, Robertson JD, Casteel SJ, Jurisson S, Smith C, Boote E, Katti KV. Nanocompatible Chemistry toward Fabrication of Target-Specific Gold Nanoparticles. J. Am. Chem. Soc. 2006,128(35):11342-11343. doi:10.1021/ja063280c.

31. Kattumuri V, Katti K, Bhaskaran S, Boote EJ , Casteel SW, Fent GM, Robertson DJ , Chandrasekhar M, Kannan R, Katti KV. Gum arabic as a phytochemical construct for 
the stabilization of gold nanoparticles: In vivo pharmacokinetics and X-ray-contrastimaging studies. Small. 2007;3(2):333-341. doi:10.1002/smll.200600427.

32. Curry F-RE. Redefining tumour vascular barriers. Nat Nanotechnol. 2016;11:494.

33. Jahnert T, Hager MD, Schubert US. Application of phenolic radicals for antioxidants, as active materials in batteries, magnetic materials and ligands for metal-complexes. $J$. Mater. Chem. A . 2014:15234-15251. doi:10.1039/c4ta03023k.

34. Mikulski D, Szela M, Molski M, Górniak R. Quantum-chemical study on the antioxidation mechanisms of trans-resveratrol reactions with free radicals in the gas phase, water and ethanol environment. J. Mol. Struct. 2010;951:37-48. doi:10.1016/j.theochem.2010.04.005.

35. Nazarparvar E, Zahedi M, Klein E. Density Functional Theory (B3LYP) Study of Substituent E ff ects on O-H Bond Dissociation Enthalpies of trans -Resveratrol Derivatives and the Role of Intramolecular Hydrogen Bonds. J Org Chem. 2012;16;77(22):10093-104. doi:10.1021/jo301612a.

36. Chatterjee S, Olsen S, Blanch EW, Wang F. Resveratrol's Hidden Hand: A Route to the Optical Detection of Biomolecular Binding. J. Phys. Chem. B, 2018, 122 (11):2841-2850. doi:10.1021/acs.jpcb.7b10278.

37. Frau J, Muñoz F, Glossman-mitnik D. A Molecular Electron Density Theory Study of the Chemical Reactivity of Cis- and Trans-Resveratrol. Molecules. 2016, 21(12), 1650. doi:10.3390/molecules21121650 
38. Gamal-Eldeen AM, Moustafa D, El-Daly SM, El-Hussieny EA, Saleh S, Khoobchandani M, Bacon KL, Gupta S, Katti K, Shukla R, Katti KV. Photothermal therapy mediated by gum Arabic-conjugated gold nanoparticles suppresses liver preneoplastic lesions in mice. J Photochem Photobiol B Biol. 2016;163:47-56. doi:10.1016/j.jphotobiol.2016.08.009.

39. Gold-smith F, Fernandez A, Bishop K. Mangiferin and Cancer: Mechanisms of Action. Nutrients. 2016, 8(7), 396. doi:10.3390/nu8070396.

40. Soto BL, Hank JA, Van De Voort TJ, Subramanian L, Polans AS, Rakhmilevich AL, Yang RK, Seo S, Kim K, Reisfeld RA, Gillies SD, Sondel PM. The anti-tumor effect of resveratrol alone or in combination with immunotherapy in a neuroblastoma model. Cancer Immunol Immunother. 2011;60(5):731-738. doi:10.1007/s00262-011-0971-0.

41. Liu H, Pan C, Yang W, Liu X. Antitumor and immunomodulatory activity of resveratrol on experimentally implanted tumor of $\mathrm{H} 22$ in Balb/c mice. World $J$ Gastroenterol. 2003;9(7):1474-1476.

42. Leischner C, Burkard M, Pfeiffer MM, Lauer UM, Busch C, Venturelli S. Nutritional immunology: function of natural killer cells and their modulation by resveratrol for cancer prevention and treatment. Nutr J. 2016:1-12. doi:10.1186/s12937-016-0167-8.

43. Park EJ, Pezzuto JM. The pharmacology of resveratrol in animals and humans. Biochim Biophys Acta - Mol Basis Dis. 2015;1852(6):1071-1113. doi:10.1016/j.bbadis.2015.01.014.

44. Carter LG, D’Orazio JA, Pearson KJ. Resveratrol and cancer: Focus on in vivo evidence. 
Endocr Relat Cancer. 2014;21(3). doi:10.1530/ERC-13-0171.

45. Tarapore RS, Siddiqui IA. Modulation of Wnt/b-catenin signaling pathway by bioactive food components. 2012;33(3):483-491. doi:10.1093/carcin/bgr305.

46. Thakur R, Mishra DP. Pharmacological modulation of beta-catenin and its applications in cancer therapy Role of -catenin in cancer. J Cell Mol Med. 2013;17(4):449-456. doi:10.1111/jcmm.12033.

47. De D, Toulouse DE. Doctorat de l' université de toulouse. 2015:1-190.

48. Fallourd MJ, Viscione L. Chapter 1 - Ingredient selection for stabilisation and texture optimisation of functional beverages and the inclusion of dietary fibre. In: Paquin PBT-F and SBT, ed. Woodhead Publishing Series in Food Science, Technology and Nutrition. Woodhead Publishing; 2009:3-38. doi:https://doi.org/10.1533/9781845695569..

49. Featherstone S. Chapter 8 - Ingredients used in the preparation of canned foods. In: Featherstone SBT-ACC in C and RP (Fourteenth E, ed. Oxford: Woodhead Publishing; 2015:147-211. doi:https://doi.org/10.1016/B978-0-85709-678-4.00008-7.

50. Sulieman AME-H. Chapter 13 - Gum Arabic as Thickener and Stabilizing Agents in Dairy Products. In: Mariod AABT-GA, ed. Academic Press; 2018:151-165. doi:https://doi.org/10.1016/B978-0-12-812002-6.00013-0.

51. Estevinho BN, Rocha F. Chapter 7 - Application of Biopolymers in Microencapsulation Processes. In: Grumezescu AM, Holban AMBT-B for FD, eds. Handbook of Food Bioengineering. Academic Press; 2018:191-222. doi:https://doi.org/10.1016/B978-0-12- 
811449-0.00007-4.

52. Han J, Schmidt A, Zhang T, Permentier H, Groothuis GMM, Bischoff R, Kuhn FE, Horvatovich P, Casini A. Bioconjugation strategies to couple supramolecular exofunctionalized palladium cages to peptides for biomedical applications. Chem Commun. 2017;53(8):1405-1408. doi:10.1039/C6CC08937B.

53. Tsai MJ, Lu IJ, Fu YS, Fang YP, Huang YB, Wu PC. Nanocarriers enhance the transdermal bioavailability of resveratrol: In-vitro and in-vivo study. Colloids Surfaces B Biointerfaces. 2016;148:650-656. doi:https://doi.org/10.1016/j.colsurfb.2016.09.045.

54. Chiou Y, Li S, Ho C, Pan M. Prevention of Breast Cancer by Natural Phytochemicals: Focusing on Molecular Targets and Combinational Strategy. Mol Nutr Food Res. 2018;1800392. doi:10.1002/mnfr.201800392. 


\section{CHAPTER 3}

\section{MATERIALS AND METHODS}

\subsection{Materials}

All chemicals and reagents used in the synthesis of palladium nanoparticles (PdNPs) were purchased from standard vendors: Sodium tetrachloropalladate (II) $\left(\mathrm{Na}_{2} \mathrm{PdCl}_{4}\right)$; resveratrol (Res), cisplatin and etoposide (Sigma-Aldrich, St. Louis, MO, USA) and gum arabic (GA) (Thermo Fisher Scientific, NJ, USA). ICP-element standard: $\mathrm{Pd}\left(\mathrm{NO}_{3}\right)_{2}$ (purity: $99.99 \%$ ), Media: Roswell Park Memorial Institute (RPMI)-1640, Hank's Balanced Salt Solution (HBSS), fetal bovine serum (FBS), TryplE, Trypan blue, MTT (3-(4,5-Dimethylthiazol-2-yl)-2,5-Diphenyltetrazolium Bromide), DAPI (4',6-diamidino-2-phenylindole), Wheat germ agglutinin (WGA), Oregon Green® 488 conjugate were obtained from ThermoFisher Scientific, USA.

\subsection{Cell line used in this study}

Cell lines used in this study included prostate cancer (PC-3) cells that were obtained from the American Type Culture Collection (ATCC) (Manassas, VA, USA), and cultured by the University of Missouri Cell and Immunobiology Core facility using procedures recommended by ATCC. Primary human aortic endothelial cells (HAECs) were used in the study as the normal control cell line. The PC-3 cells were maintained in RPMI-1640 media supplemented with [+] Lglutamine, $10 \%$ FBS and $0.7 \mathrm{~mL}$ gentamicin, and HAEC cells were maintained in vascular cell basal medium supplemented with endothelial cell growth kit-VEGF (ATCC, Manassas, VA, USA). 


\subsection{Characterization of palladium nanoparticles (PdNPs)}

The absorption measurements were collected by using the Cary $60 \mathrm{UV}$-vis spectrophotometer, Agilent Technologies, Santa Clara, USA. A Malvern Zetasizer Nano-ZS90, Malvern Instruments Ltd., UK using dynamic light scattering (DLS) was used to determine the hydrodynamic size and zeta potential. Eppendorf 5424R centrifuge (Eppendorf AG, Hamburg, Germany) was used to purify the PdNPs and the concentration of Pd was measured by Inductively coupled plasma optical emission Spectroscopy (ICP-OES), Perkin Elmer, MA, USA while the quantification of Res molecules conjugated to the surface of the PdNPs was investigated using liquid chromatography-tandem mass spectrometry (LC-MS/MS) with multiple reaction monitoring (MRM) (Thermo Fisher Scientific, Waltham, MA). Cellular viability studies were measured using the Molecular device SpectraMax M2 microplate reader at $570 \mathrm{~nm}$, CytoViva Nanoscale Hyperspectral Microscopy, (CytoViva, Inc., Auburn, USA), the Leica SP8 spectral confocal microscope (Leica microsystems, Wetzlar, Germany) was used to investigate cellular internalization of the PdNPs, and Olympus 1X71 Fluorescent Microscope, (Olympus, Tokyo, Japan) was used to study cellular morphological changes of the cells. The acquisition of Transmission Electron Microscope (TEM) images was by loading the PdNPs onto a carboncoated copper grid (carbon square 200 mesh (CF200-CU-UL), Hatfield, PA, Electron microscopy sciences (EMS), USA) and the sample-loaded grid was subsequently air-dried. TEM images were acquired with JEOL 1400 TEM operated at $300 \mathrm{kV}$ (JEOL, Ltd, Tokyo, Japan). Elemental studies were obtained on EI Tecnai F30 G2 Twin TEM with HAADF STEM coupled to an energy-dispersive X-ray spectroscopy (EDS), JEOL, LTD., Tokyo, Japan. The functional groups of Res conjugated to the surface of PdNPs were characterized by Fourier-transform infrared spectroscopy (FT-IR). The FT-IR spectra were acquired with a Varian 640-IR (Agilent Technologies, Santa Clara, CA, USA). 
3.4 Synthesis of resveratrol-derived phenols encapsulated palladium nanoparticles (ResPdNPs)

The synthesis of palladium nanoparticles was prepared as shown in Scheme 2.

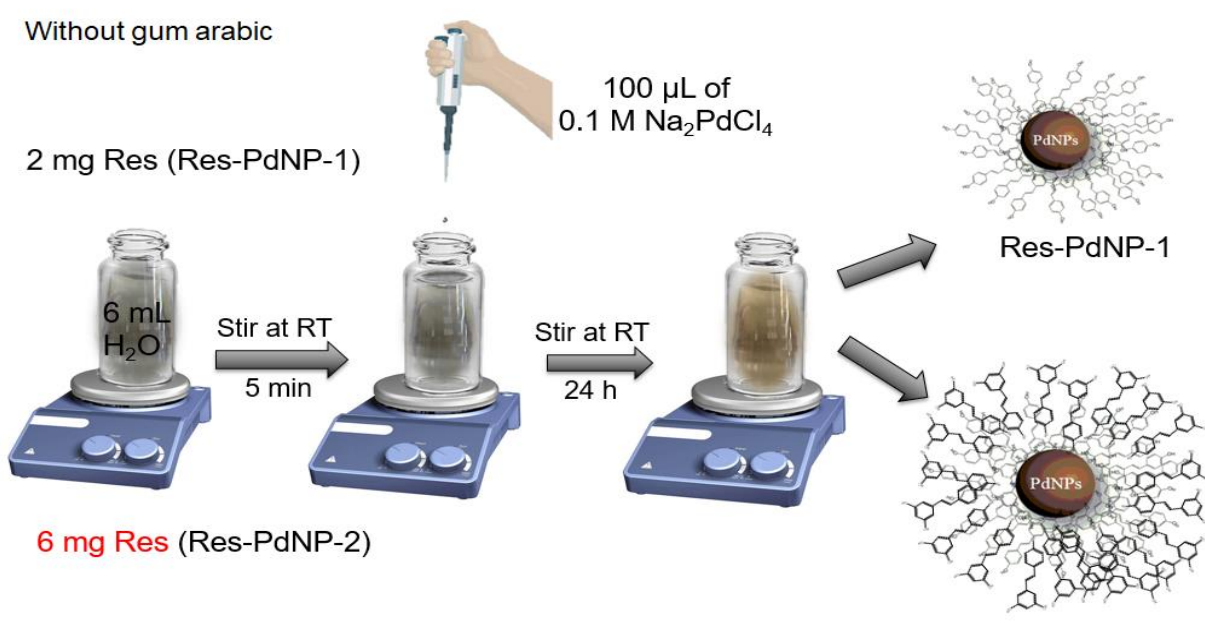

Res-PdNP-2

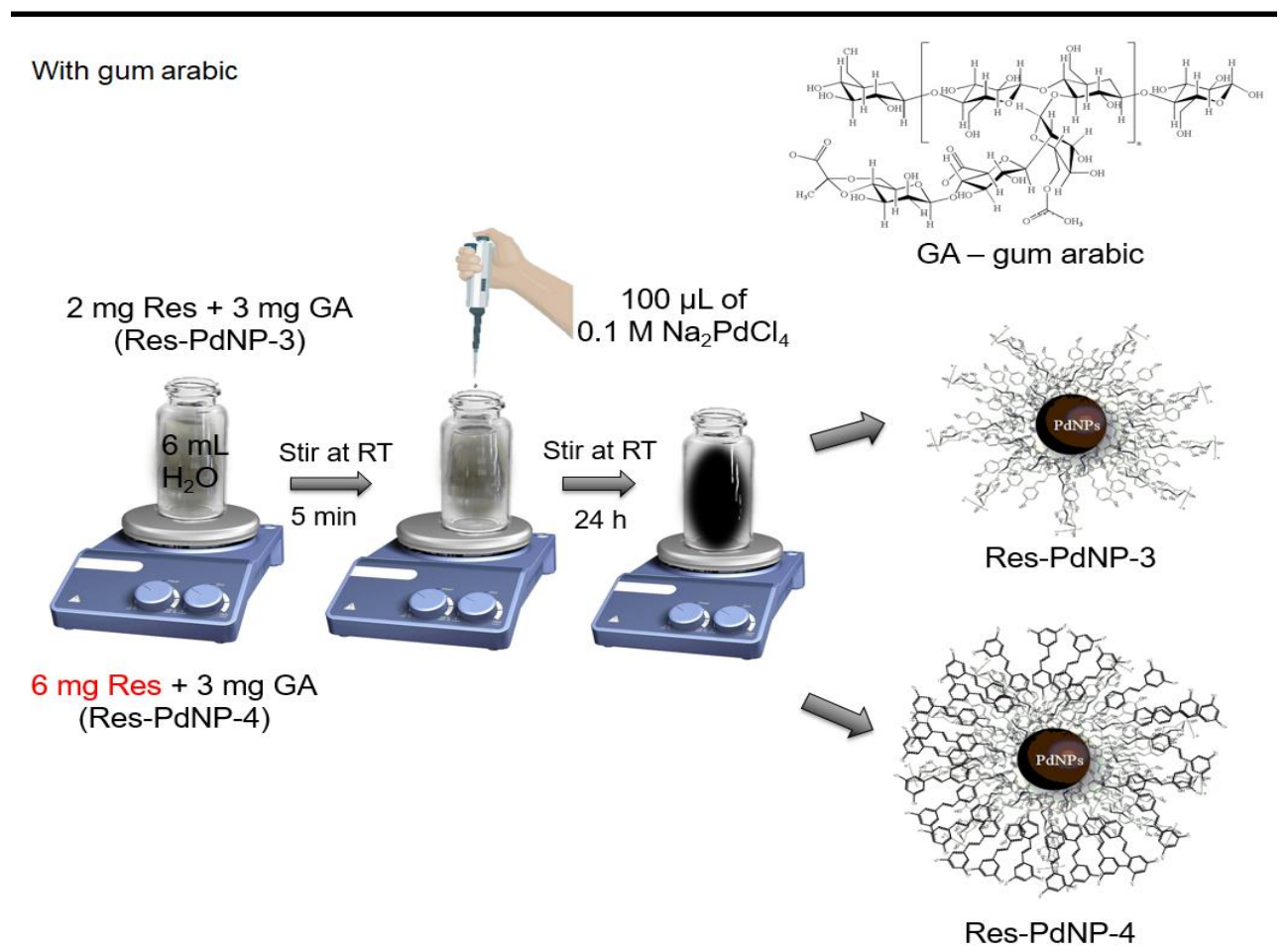

Scheme 2: Synthesis of PdNPs using resveratrol as a reducing and capping agent. RT room temperature 


\subsubsection{Res initiated and stabilized palladium nanoparticles (Res-PdNP-1 and Res-PdNP-2)}

The production of Res-PdNPs (Res-PdNP-1 and Res-PdNP-2) was carried out by varying the amount of Res (from 0.5 to $8 \mathrm{mg}$ ) dissolved in $6 \mathrm{~mL}$ of distilled water and stirred, to this mixture $100 \mu \mathrm{L}$ of two concentrations of $\mathrm{Na}_{2} \mathrm{PdCl}_{4}(10 \mathrm{mM}$ and $0.1 \mathrm{M})$. After completing rigorous extensive optimization and reproducibility studies. The following synthetic routes were achieved:

Res-PdNPs were synthesized by adding $2 \mathrm{mg}$ of Res into a $20 \mathrm{~mL}$ vial and $6 \mathrm{~mL}$ of distilled water were added. The mixture was stirred for $5 \mathrm{~min}$ at room temperature. To the mixture, 100 $\mu \mathrm{L}$ of $0.1 \mathrm{M} \mathrm{Na}_{2} \mathrm{PdCl}_{4}$ salt was added for the production of Res-PdNP-1. An attempt to increase the Res-derived phenols and polyphenols corona on the PdNPs, $6 \mathrm{mg}$ of Res was used for the synthesis while other reaction conditions remained consistent, for the production of Res-PdNP-2. The color of the solutions changed from a white to a dark brown solution, which indicated the formation of PdNPs and the solution was stirred overnight for optimization. The Res-PdNPs solutions were washed twice; by ultracentrifugation at $17000 \mathrm{rpm}$ at $12{ }^{\circ} \mathrm{C}$ for $15 \mathrm{~min}$ using an Eppendorf 5424R centrifuge (Eppendorf AG, Hamburg, Germany) and re-suspended in distilled water.

\subsubsection{Res initiated and gum arabic stabilized palladium nanoparticles (Res-PdNP-3 and Res-PdNP-4}

The production of Res-PdNP-3 and Res-PdNP-4 was facilitated through the addition of different ratios of gum arabic (GA) to Res $(0.5: 1,1: 1,1.5: 1$ and 2:1). The synthetic routes were optimized to achieve the reproducibility of Res-PdNP-3 and Res-PdNP-4. The Res-PdNPs were synthesized 
by adding $2 \mathrm{mg}$ of Res and $3 \mathrm{mg}$ of GA into a $20 \mathrm{~mL}$ vial and $6 \mathrm{~mL}$ of distilled water were added. The mixture was stirred for $5 \mathrm{~min}$ at room temperature. One hundred $\mu \mathrm{L}$ of $0.1 \mathrm{M}$ $\mathrm{Na}_{2} \mathrm{PdCl}_{4}$ salt was added to the mixture to produce Res-PdNP-3. On the other hand, $6 \mathrm{mg}$ of Res was used to increase the Res-derived phenols and polyphenols corona on the PdNPs for the production of Res-PdNP-4. The color of the solutions changed from a white solution to a dark black colored solution, which indicated the formation of PdNPs and the solution was stirred overnight. The PdNPs solution was washed twice by ultracentrifugation at $17000 \mathrm{rpm}$ at $12{ }^{\circ} \mathrm{C}$ for $15 \mathrm{~min}$ and re-suspended in distilled water.

Control PdNPs were produced using GA: Briefly, $12 \mathrm{mg} \mathrm{GA}$ was dissolved in $6 \mathrm{~mL}$ of distilled water and stirred at $100{ }^{\circ} \mathrm{C}$ for $5 \mathrm{~min}$. To this mixture, $100 \mu \mathrm{L}$ of $0.1 \mathrm{M} \mathrm{Na}_{2} \mathrm{PdCl}_{4}$ solution was added for the production of gum arabic stabilized palladium nanoparticles (GA-PdNPs).

\subsection{Characterization of PdNPs}

\subsubsection{In vitro stability analysis of PdNPs}

The stability of Res-PdNP-1, Res-PdNP-2, Res-PdNP-3 and Res-PdNP-4 was further confirmed by mixing the $1 \mathrm{~mL}$ of PdNPs with $2 \mathrm{~mL}$ of various biological solutions in triples $(\mathrm{n}=3)$ such as aqueous solutions of $1 \% \mathrm{NaCl}, 0.5 \%$ cysteine, $0.2 \mathrm{M}$ histidine, $0.5 \%$ human serum albumin (HSA), $0.5 \%$ bovine serum albumin (BSA), and $\mathrm{pH} \mathrm{5,} \mathrm{pH} \mathrm{7,} \mathrm{pH} 9$ and a mixture of these solutions at equivalent volumes was incubated at $37{ }^{\circ} \mathrm{C}$ to mimic in vivo condition, respectively. The samples were measured by DLS using the Malvern Zetasizer Nano-ZS90 to monitor the change in the hydrodynamic size as a parameter for stability, this was measured for a week. 


\subsubsection{Determination of palladium nanoparticles concentration}

The concentration of PdNPs can be determined by calculation using a geometric method and experimentally using inductively coupled plasma atomic emission spectroscopy (ICP-OES).

\section{(i) Geometric methods}

The crystallization of palladium nanoparticles in each unit cell consists of 8 corners: 1/8 and 6 faces: $1 / 2$, therefore a unit cell has $(8 \times 1 / 8)+(6 \times 1 / 2)=4$ atoms as shown in Scheme 3 .
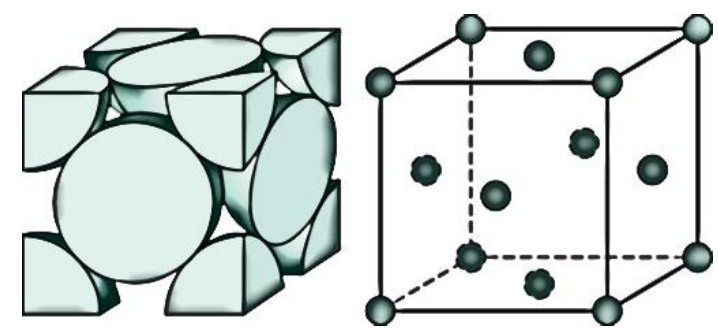

\section{Scheme 3: Unit cell of a face centered cubic (FCC) crystalline palladium nanoparticle}

The radius of a Pd atom can be calculated, given that Pd has an FCC crystal structure, a density (d) of $12.0 \mathrm{~g} / \mathrm{cm}^{3}$, and an atomic weight $(M)$ of $106.4 \mathrm{~g} / \mathrm{mol}, N_{A}$ is Avogadro's number (per mol) is $6.022 \times 10^{23}, Z$ is the number of atoms per unit cell $=4, a$ is the edge length and $r$ is the atomic radius in Eq. 1 and 2. ${ }^{211-213}$

$$
d=\frac{Z \times M}{a^{3} \times N_{A}}
$$

Eq. 1

Relation between atomic radius(r) and edge length (a) is given by: 


$$
\begin{gathered}
r=\frac{\sqrt{2} a}{4} \Rightarrow \\
a^{3}=\frac{Z \times M}{d \times N_{A}} \\
\left(\frac{4 r}{\sqrt{2}}\right)^{3}=\frac{Z \times M}{d \times N_{A}}
\end{gathered}
$$

Eq. 2

Solve for $r$

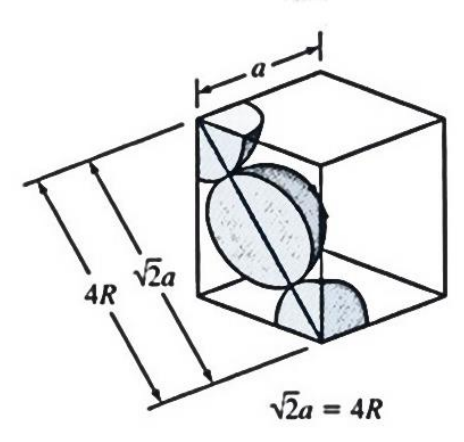

$$
\begin{aligned}
& \left(\frac{4 r}{\sqrt{2}}\right)^{3}=\frac{4 \times 106.4}{12 \times\left(6.022 \times 10^{23}\right)} \\
& \frac{64 r^{3}}{2 \sqrt{2}}=5.88 \times 10^{-23} \\
& r^{3}=2.59 \times 10^{-23} \\
& r=1.37 \times 10^{-8} \mathrm{~cm} \\
& r=0.1375
\end{aligned}
$$

Since the atomic radius for $\mathrm{Pd}=0.1375 \mathrm{~nm}$, the volume $\left(V_{c}\right)$ of the unit cell is calculated using Eq. 3, were $a$ is the edge length

$$
\begin{gathered}
V_{c}=a^{3} \\
a=\frac{4 r}{\sqrt{2}}
\end{gathered}
$$




$$
\begin{gathered}
V_{c}=\left(\frac{4 r}{\sqrt{2}}\right)^{3} \\
V_{c}=\left(\frac{4 \times 0.1375}{\sqrt{2}}\right)^{3} \\
=0.05877
\end{gathered}
$$

\section{Atomic packing}

Spherical palladium nanoparticles:

$$
\begin{gathered}
V=\frac{4}{3} \pi r^{3} \\
\frac{V_{\text {per atom }}}{V_{\text {per cell }}}=\frac{4}{3} \pi r^{3} \quad \mathrm{~V}_{\text {per atom }}=1.6286 \mathrm{r}^{3}
\end{gathered}
$$

Atomic packing factor can be calculated using Eq. 4:

$$
\begin{aligned}
& \mathrm{APF}=\frac{V_{\text {atom }}}{V_{\text {crystal }}}=\frac{\frac{4}{3} \pi r^{3}}{0.05877} \\
& ={ }_{71.274} r^{3} \\
& \mathrm{~N}=\mathrm{n} \times(\text { APF })
\end{aligned}
$$$$
\text { Eq. } 4
$$

Where $\mathrm{n}-$ number of atoms/unit cell $\mathrm{n}=4$ 
Final derivation relies solely on the radius or diameter of the nanoparticle core using Eq. 5 to determine the average number of palladium atoms per nanosphere:

$$
\mathrm{N}=285.1 r^{3}
$$

Where $r$ is the radius of the nanoparticle. To determine the concentration of PdNPs/mL, Eq. 6 was used, where $N_{T}$ - total number of Pd atoms divided by $\mathrm{N}$ - average number of palladium atoms per nanosphere, where $\mathrm{V}(1 \mathrm{lit})$ is the volume of the reaction solution in liter and $N_{A}$ is the Avogadro's constant $\left(6.022 \times 10^{23}\right)$. The concentrations of nanoparticles were calculated with the following formula:

$$
C=\frac{N_{T}}{N V N_{A}}
$$

Eq. 6

\section{(ii) Inductively coupled plasma - optical emission spectroscopy (ICP-OES)}

Inductively Coupled Plasma - Optical Emission Spectroscopy (ICP-OES) instrument (Model: 4100, Agilent Inc., Santa Clara, CA, USA) was used to determine the concentration of Pd in the PdNPs. The sample introduction system consisted of solvent-resistant tubing, a double-pass cyclonic chamber, and an inert flow blurring nebulizer (Nene). Acid digestion technique was utilized for the preparation of samples. PdNPs were digested in a mixture of $\mathrm{HNO}_{3}(1.1 \mathrm{~mL} \sim 65$ $\%)$ and $\mathrm{HCl}(0.3 \mathrm{~mL} \sim 30 \%)$ and diluted to $10 \mathrm{~mL}$ with ultrapure water. Three replicates of the sample and a certified reference material (ICP-element standard: $\operatorname{Pd}\left(\mathrm{NO}_{3}\right)_{2}$ (purity: $99.99 \%$ ) diluted to $1 \mathrm{mg} / \mathrm{mL}$ were analyzed. Total elemental concentrations of the PdNPs were determined by ICP-Quadrupole-MS (ICP-QMS; Agilent 7700 series, Agilent Technologies, Germany). The ICP-MS was equipped with a PEEK Mira Mist nebulizer (Burgener research, 
Canada) and a PFA inert sample introduction kit with a sapphire injector (inner diameter 2.5 mm, for Agilent 7700 series, Agilent Technologies, Germany). Measurements were conducted at a RF power of $1,550 \mathrm{~W}$ and a carrier gas flow of $1.17-1.18 \mathrm{~L} / \mathrm{min}$.

\subsection{Quantification of resveratrol on the surface of PdNPs}

\subsubsection{Liquid chromatography-tandem mass spectrometry multiple reaction monitoring (LC-MS/MRM)}

The amount of Res molecules conjugated to the surface of the PdNPs was quantified using liquid chromatography-tandem mass spectrometry (LC-MS/MS) with multiple reaction monitoring (MRM) on a Quantiva triple quadrupole mass spectrometer (Thermo Fisher Scientific, Waltham, MA). Method development was conducted to determine MRM transitions for Res and its retention time on the column. The Res-PdNPs were stored at $4{ }^{\circ} \mathrm{C}$ prior to analyses. The ResPdNPs were centrifuged at $16 \mathrm{~K} \times \mathrm{g}$ in a micro-centrifuge tube and the supernatant diluted 1:10,000 with internal standard solution $(30 \mathrm{ng} / \mathrm{mL}$ tolbutamide in $5 \%$ acetonitrile $(\mathrm{ACN}) / 1 \%$ formic acid (FA)). Then $25 \mu \mathrm{L}$ was transferred into autosampler vials and placed in a cooled (7 $\left.{ }^{\circ} \mathrm{C}\right)$ autosampler.

Res standard $(5 \mu \mathrm{L})$ was full-loop injected into a $\mathrm{C}_{18}$ trap column $\left(\mathrm{C}_{18}\right.$ trap, Eksigent) and the sample was eluted from the trap column and separated on a $20 \mathrm{~cm}$ x $75 \mu \mathrm{m}$ inner diameter pulledneedle analytical column packed with $\mathrm{HxSIL}_{18}$ reversed phase resin (Hamilton Co.) with a step gradient of acetonitrile at $500 \mathrm{~nL} / \mathrm{min}$. The Eksigent Nano 1D plus HPLC system is attached to a Thermo Scientific TSQ Quantiva triple-quadrupole mass spectrometer. LC gradient conditions: Initial conditions were $2 \% \mathrm{~B}(\mathrm{~A}: 0.1 \%$ formic acid in water, B: $99.9 \%$ acetonitrile, $0.1 \%$ formic 
acid), followed by 2 min ramp to $90 \% \mathrm{~B}$, held at $90 \%$ B for 8 min, ramped back to (1 min) and held at (2 min) initial conditions. Total run time was $10 \mathrm{~min}$.

MRM conditions: ionization voltage 1600V, Q1 \& Q3 resolution 0.7 (full-width half max), collision gas 2.5 mTorr, Dwell Time 200 msec. An MRM transition consists of a precursorfragment pair, which allowed the mass spectrometer (Thermo Scientific TSQ Quantiva QQQ) to ignore every molecule in a sample except the Res to facilitate accurate quantitation with very high specificity and sensitivity. To specifically quantify the Res, two optimized transitions were generated as follows: The stock solution of Res $(2.5 \mathrm{mg} / \mathrm{mL}$ in ethanol) was diluted 1:1000 in $70 \%$ ACN, $0.1 \%$ FA. This solution was then analyzed by direct infusion $(3 \mu \mathrm{L} / \mathrm{min})$ for quantification.

\subsection{Cellular studies of PdNPs encapsulated with Res-derived phenols and polyphenols}

\subsubsection{MTT Cell Proliferation Assay}

MTT Cell Proliferation Assay kit was obtained from ATCC, was used to determine the in vitro cellular viability of the Res-PdNPs against the cells (prostate cancer (PC-3) and primary aortic endothelial cells (HAEC) used in this study. Briefly, $100 \mu \mathrm{L} /$ well of $5 \times 10^{4}$ cells/mL were seeded into 96-well plates and the cells were incubated for $24 \mathrm{~h}$ at $37{ }^{\circ} \mathrm{C}$ in $\mathrm{CO}_{2}$ incubator with $5 \% \mathrm{CO}_{2}$. After incubation, the media was removed and the cells were treated with the Res-PdNPs, GAPdNPs, free Res and GA, while cisplatin and etoposide were used as positive controls and cells with no treatment were used as negative controls at different concentrations $(0,2.75,5.5,11,22$ and $44 \mu \mathrm{g} / \mathrm{mL})$, respectively. The cells were treated at different time intervals $(24,48$ and $72 \mathrm{~h})$ and after incubation in a quadruplet manner, $10 \mu \mathrm{L}$ of MTT dye solution was added in each well and the plates were incubated further for $4 \mathrm{~h}$ to allow crystallization. The media solution was 
carefully removed and $100 \mu \mathrm{L}$ of dimethyl sulfoxide (DMSO) was added to dissolve the formazan crystals. The cell viability was measured by microplate reader (Molecular device, USA) operating at $570 \mathrm{~nm}$. The percent cell viability was calculated by using the formula:

$$
\text { Cellular viability }(\%)=\frac{\text { Absorbance of the treated cells at } 570 \mathrm{~nm}}{\text { Absorbance of untreated cells at } 570 \mathrm{~nm}} \times 100 \%
$$

The half-maximal inhibitory concentration $\left(\mathrm{IC}_{50}\right)$ values were calculated using GraphPad Prism version 6.01 software.

\subsubsection{Migration assay}

The 6-well plates were marked to monitor the region for acquiring an image and $5 \times 10^{5}$ cells $/ \mathrm{mL}$ was seeded into 6-well plates and the cells were incubated for $24 \mathrm{~h}$ for $100 \%$ confluence. A 200 $\mu \mathrm{L}$ pipette tip was used to aseptically make a vertical stretch though the cell monolayer. The media and cell debris were carefully aspirated and the plates were gently watched once with $1 \mathrm{x}$ PBS solution. The cells were treated with highest concentration of Res-PdNP-4 (44 $\mu \mathrm{g} / \mathrm{mL})$ for 24 h. The Olympus 1X71 Fluorescence Microscope (Shinjuku, Tokyo, Japan) was used to acquire images of the cells at 1 and $24 \mathrm{~h}$ after treatment. The area of the stretch was measured using the CellSens Dimension Software 1.13.

\subsubsection{Cellular internalization and trafficking pathway}

The cellular internalization mediated endocytosis mode of Res-PdNPs was investigated by blocking the laminin receptor (67LR) overexpressed onto prostate and various other tumor cells. The optimum dose and incubation time was determined by incubating the cells with highest concentration of Res-PdNP-4 (44 $\mu \mathrm{g} / \mathrm{mL}$ ) for different time points (6 and $24 \mathrm{~h}$ ). Cell trafficking 
pathway of Res-PdNPs was further evaluated by using RPSA Monoclonal Antibody (MLuC5) inhibitor to confirm laminin receptor mediated endocytosis.

This experiment was performed by two techniques - confocal fluorescence microscope and TEM. For dark field and confocal microscopic studies, ultra clean and sterile cover slips were kept in 6 well plate to grow the cells on the cover slip. For the study by TEM technique, cells were grown on the plate without the coverslip. Briefly, $8 \times 10^{5}$ cells $/ \mathrm{mL}$ were seeded into 6 well plates in the respective media and incubated for $24 \mathrm{~h}$ in $\mathrm{CO}_{2}$ incubator at $37^{\circ} \mathrm{C}$. The cells were pre-incubated with the inhibitors as following: PBS (control) and MLuC5 laminin receptor antibody $(10 \mu \mathrm{g} / \mathrm{mL} ; 60 \mathrm{~min})$ and the cells were incubated in $\mathrm{CO}_{2}$ incubator at $37^{\circ} \mathrm{C}$. After the incubation, cells are washed twice with 1x PBS followed by the treatment with Res-PdNP-4 (44 $\mu \mathrm{g} / \mathrm{mL}$ ) for $6 \mathrm{~h}$ in $\mathrm{CO}_{2}$ incubator at $37^{\circ} \mathrm{C}$. The samples were prepared for the following techniques:

\subsubsection{Confocal and dark-field microscopic technique}

After incubation, cells were washed five times with 1x PBS and fixed with $4 \%$ paraformaldehyde (PFA) and further incubated for 15 min in the dark. The cells were washed three times with 1x HBSS and cells were labeled with $2 \mu \mathrm{g} / \mathrm{mL}$ WGA conjugate, incubated for $10 \mathrm{~min}$ at room temperature in the dark. After incubation, the cells were washed twice with 1x HBSS, thereafter, the cells were permeabilized with $0.2 \%$ Triton $\mathrm{X}-100$ for $5 \mathrm{~min}$ at room temperature. The cells were further washed twice with 1x HBSS and the slides were prepared by using DAPI nuclear dye and observed with CytoViva Nanoscale Hyperspectral Microscopy coupled with dual mode fluorescence (CytoViva, Inc., Auburn, USA) and the Leica SP8 spectral confocal 
microscope. Cell morphology was initially observed, followed by the uptake of Res-PdNP-4 and the images were captured via Dage Imaging Software and LAS X software, respectively.

\subsubsection{Transmission electron microscopy (TEM) technique}

This technique provided an overview of the Res-PdNP-4 uptake in the each cell compartment. After incubation, cells were washed 10 times with 1x PBS solution, centrifuged into pellets, and fixed with $2 \%$ glutaraldehyde $2 \%$ paraformaldehyde in sodium cacodylate buffer $(0.1 \mathrm{M})$ incubated for $15 \mathrm{~min}$ in the dark. The cells were further post-fixed with $1 \%$ buffered osmium tetraoxide in 2-mercaptoethanol buffer and dehydrated in graded acetone series and embed in Epon-Spurr epoxy resin. Sections were cut at $85 \mathrm{~nm}$ using a diamond knife (Diatom, Hatfield PA, USA). The sections were stained with Sato's triple lead stain and 5\% aqueous uranyl acetate for organelle visualization. The prepared samples were examined on JEOL 1400 TEM microscope (JEOL, Peabody, Mass.) operated at $80 \mathrm{kV}$ at the University of Missouri's Electron Microscopy Core Facility, Columbia, MO, USA.

\subsection{Animal studies}

The in vivo experiments of Res-PdNPs involving animals were approved by the Institutional Animal Care and Use Committees (IACUC) of the Harry S. Truman Memorial Veterans Hospital and the University of Missouri-Columbia, MO, USA. The experiments were performed according to the guidelines outlined in the Care and Use of Laboratory Animals. Imprinting control regions-severe combined immunodeficiency (ICR - SCID) male mice (from Taconic Farms, Hudson, New York) were used for the therapeutic study. The mice used in our investigations weighed 28-31 g. 


\subsubsection{In vivo therapeutic efficacy study of Res-PdNP-4}

The severely compromised immunodeficiency (SCID) male mice were subcutaneously inoculated with $10 \times 10^{6}$ prostate cancer (PC-3) cells (suspended in $0.1 \mathrm{~mL}$ of sterile DPBS and Matrigel $\left.{ }^{\circledR}(2: 1, \mathrm{v}: \mathrm{v})\right)$ in the right hind flank under inhalation anesthesia (isoflurane/oxygen). After inoculation, the animals were treated when the tumors were palpable by intravenous (IV) administration twice a week. The mice were randomly divided into three groups ( $\mathrm{n}=5 /$ group) with no significant difference in tumor volume, the day of randomization was considered day zero of therapy study. At the day 0 , tumors in the mice were measured by digital caliper measurements and calculated as length $\mathrm{x}$ width $\mathrm{x}$ height. The first group was IV injected with $100 \mu \mathrm{L}$ saline, this served as the control group. The second group was IV injected with $100 \mu \mathrm{L}$ Res-PdNP-4 (0.25 mg/kg bw) whereas the third group was IV injected with $100 \mu \mathrm{L}$ free Res ( 7 $\mathrm{mg} / \mathrm{kg}$ bw) as shown in Table 5 .

Table 5: Antitumor study dose design for the animal body weight (kg)

\begin{tabular}{|l|l|l|l|}
\hline Group & Sample & Dose $(\mathbf{m g} / \mathbf{k g ~ b w})$ & Number of mice \\
\hline 1 & Saline control & -- & 5 \\
\hline 2 & Res-PdNP-4 IV & 0.25 & 5 \\
\hline 3 & Free Res & 7 & 5 \\
\hline
\end{tabular}

${ }^{\mathrm{IV}}$ Intravenous administration 
The animals were monitored for their tumor volume, body weight and health effects for another 6-7 weeks and were sacrificed at the end of the study. A normal healthy group of mice $(n=5)$ that had not been experimentally manipulated served as control for complete blood counts (CBC) parameters (mean counts for white blood cells (WBC), red blood cells (RBC) as well as

hemoglobin, lymphocytes, and platelets counts) and body weight/eating habits of animals to monitor the tolerated of the treated groups. Body weight and tumor volume measurements were observed twice per week for all groups, for approximately 6-7 weeks. Animals were sacrificed at the end of study or when tumors reached endpoint (when animals were experiencing pain, distress and illness through the evaluation of body weight, physical appearance, measurable clinical signs, unprovoked behavior, response to external stimuli and a tumor burden greater than $10 \%$ body weight. Tumors were submitted for histology for the angiogenesis measurements. For angiogenesis analysis, animal tissues were stained with CD31 antibody.

\subsection{Statistical analysis}

All experimental data were given as mean \pm SEM. Statistical analysis was carried out using the one-way analysis of variances (ANOVA) using Graph Pad Prism software. $P<0.05$ was considered significant. 


\section{CHAPTER 4}

\section{RESULTS AND DISCUSSIONS}

\subsection{UV-Vis spectroscopy of palladium nanoparticles (PdNPs) encapsulated with resveratrol (Res)-derived phenols and polyphenols}

\subsubsection{Res initiated and stabilized palladium nanoparticles (Res-PdNP-1 and Res-PdNP-2)}

Resveratrol was utilized to produce palladium nanoparticles. Res-derived phenols and polyphenols encapsulated palladium nanoparticles were synthesized through a redox reaction by transferring electrons from hydroxyl groups of Res to the palladium salt. The formation of PdNPs was observed by a color-change from a colorless solution to a brown color solution. The chemical structure of the Res plays a significant role as a powerful electron injector that also creates coating on palladium nanoparticles surface. The resulting solution of nanoparticles (ResPdNP-1 and Res-PdNP-2 (increased Res-derived phenols and polyphenols corona)) was characterized by measuring the morphology, size distribution and surface charge determined by ultraviolet-visible (UV-Vis) spectroscopy, transmission electron microscopy (TEM) and dynamic light scattering (DLS), respectively.

The UV-vis spectra of Res showed an absorption peak at $306 \mathrm{~nm}$, representing transresveratrol. ${ }^{1-3}$ The UV -vis spectra of the metal precursor $\left(\mathrm{Na}_{2} \mathrm{PdCl}_{4}\right)$ has an absorption peak at $420 \mathrm{~nm}$ attributed by $\mathrm{Pd}^{2+}$ ions. ${ }^{4,5}$ The UV-Vis spectra of the Res-PdNP-1 and Res-PdNP-2 solution revealed the disappearance of $420 \mathrm{~nm}$ absorbance, which confirmed the reduction of the $\mathrm{Pd}^{2+}$ to $\mathrm{Pd}^{0}$, thereby confirming the synthesis of PdNPs (Figure 13). 

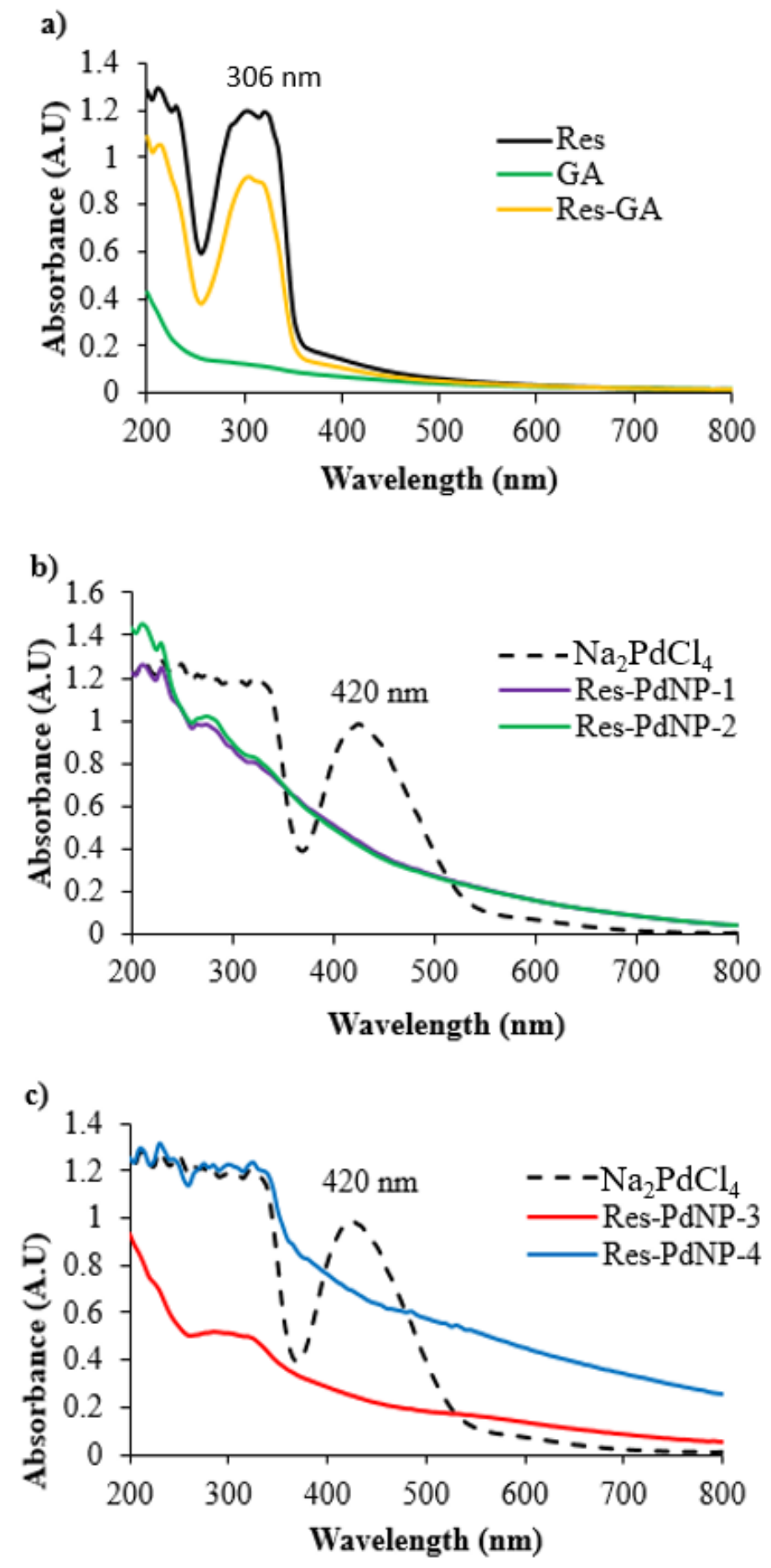

Figure 13: UV-Vis spectra of palladium nanoparticles encapsulated with resveratrolderived phenols and polyphenols a) free Res, GA and Res-GA mixture, b) Res-PdNP-1 and Res-PdNP-2 and c) Res-PdNP-3 and Res-PdNP-4. 


\subsubsection{Res initiated and Gum Arabic stabilized palladium nanoparticles (Res-PdNP-3 and Res-PdNP-4)}

The color-change from a colorless solution to a dark-brown color solution and the disappearance of the $420 \mathrm{~nm}$ peak on the UV-Vis spectra of GA stabilized PdNPs revealed the successful production of PdNPs. ${ }^{6}$ The absorption peak at $306 \mathrm{~nm}$ of the Res-PdNP-3 and Res-PdNP-4 (increased Res corona) indicated Res-derived phenols and polyphenols encapsulation on the surface of the PdNPs. The intensity of the Res peak was higher in Res-PdNP-4 as compared to Res-PdNP-3 which was due to increased Res-derived phenols and polyphenols corona on the surface of the PdNPs as shown in Figure 13. Moreover, The UV-Vis spectra of the Res-GA mixture (Figure 13) predominantly showed the $306 \mathrm{~nm}$ absorption peak reflected by Res molecule anchoring onto the structure of GA. This data revealed that GA provides a supportive matrix to stabilize nanoparticles and increase the loading of Res-derived phenols and polyphenolic molecules. $^{7}$

The physicochemical properties of the synthesized PdNPs were further evaluated by dynamic light scattering (DLS) and transmission electron microscopy (TEM). The DLS was used to determine the hydrodynamic size and zeta $(\zeta)$ potential of Res-PdNPs. The DLS measurements revealed that the hydrodynamic sizes of Res-PdNP-1, Res-PdNP-2, Res-PdNP-3 and Res-PdNP4 were $93 \pm 4,254 \pm 18,44 \pm 1$, and $117 \pm 4 \mathrm{~nm}$, respectively, as shown in Table 6 . It was expected that Res-PdNP-2 and Res-PdNP-4 would have a significantly larger hydrodynamic size compared to Res-PdNP-1 and Res-PdNP-3 due to increased Res-derived phenols and polyphenols corona on the surface of the PdNPs due to the surface coating (Table 6). ${ }^{7-9}$ The $\zeta$ potential provides crucial information of inter-particle and nanoparticle-cell interaction on the stability of nanoparticles in solution. The magnitude of the $\zeta$-potential (hydrodynamic shear 
force) indicates the degree of electrostatic repulsive forces between adjacent particles in fluid environment of the blood resulting in low serum protein binding and potentially longer circulation time. ${ }^{10}$ This provides an excellent index for the efficient dispersion and the long-term stability for both in vitro and in vivo systems for the application of PdNPs in biomedical and other applications.

The $\zeta$-potentials were $-26 \pm 2,-28 \pm 3,-31 \pm 1$ and $-40 \pm 3 \mathrm{mV}$ for Res-PdNP-1, Res-PdNP-2, ResPdNP-3 and Res-PdNP-4, respectively (Table 6). A higher $\zeta$-potential is directly proportional to the stability of the nanoparticles, indicating no tendency of the particles to aggregate. ${ }^{11}$ The anionic carboxyl groups of GA offer additional stability as shown by a higher $\zeta$-potential compared to Res-PdNPs without GA. ${ }^{12}$ The polydispersity index (PDI) indicates the quality of the nanoparticles with respect to the average uniformity (shape and size) of a particle solution, whether the nanoparticles are monodispersed or polydispersed on the basis of size distribution, which is a highly important physical characteristic of pharmaceutical-grade products. ${ }^{13}$ The FDA's Guidance for Industry emphasizes the importance of particle size distribution (polydispersity) as nanomaterial's critical quality attributes (CQAs) ${ }^{13}$ According to Danaei et al. ${ }^{13}$ in drug delivery applications using lipid-based carriers (liposome and nanoliposome formulations, such as Doxil®, Abraxane®, NanoTherm ${ }^{\circledR}$, Ferumoxtran-10, and CellSearch ${ }^{\circledR}$ ), PDI values that are less than 0.3 are acceptable for clinical applications. The PDI of the synthesized PdNPs indicated that Res-PdNP-1, Res-PdNP-3 and Re-PdNP-4 was 0.3, which indicate monodispersity; the PDI of Res-PdNP-2 was at 0.5 reflecting some degree of polydispersity as shown in Table 6 . 


\subsection{Electron microscopy studies of Res-PdNPs}

The TEM images revealed that Res-PdNPs were monodispersed and are spherical in shape as shown in Figure 14. The size distribution for the measurement of the core size was performed by the analysis of the TEM images using the ImageJ software, and showed that the average core sizes of Res-PdNP-1, Res-PdNP-2, Res-PdNP-3 and Res-PdNP-4 are 83 $\pm 14,53 \pm 14,24 \pm 6$ and $9 \pm 3 \mathrm{~nm}$, respectively (Figure 14 and 15). For the Res-PdNPs with GA (Res-PdNP-3 and ResPdNP-4), one would expect a higher size, however the arabinogalactan proteins of GA have been reported as reducing and stabilizing agents for the synthesis of nanoparticles. ${ }^{14}$ Similarly Moreno-Trejo and Sánchez-Domínguez ${ }^{14}$, reported on the use of Mesquite Gum (proteinaceous arabinogalactan gum that is similar to GA) as a reducing and stabilizing agent for the synthesis of highly concentrated silver nanoparticles. Moreover, these proteins give some physicochemical properties (immunochemical identity and high solubility in water) to the GA to form compact coacervation of globular structures; these structures in aqueous solution provide the capacity to reduce the surface tension and to act as a steric stabilizer. ${ }^{15}$ The TEM image of Res-PdNP-2 shows that the nanoparticles seem to be denser compared to the Res-PdNP-1, and this can be attributed to amount of loaded Res-derived phenols and polyphenolic molecules as concurred with the DLS data. 

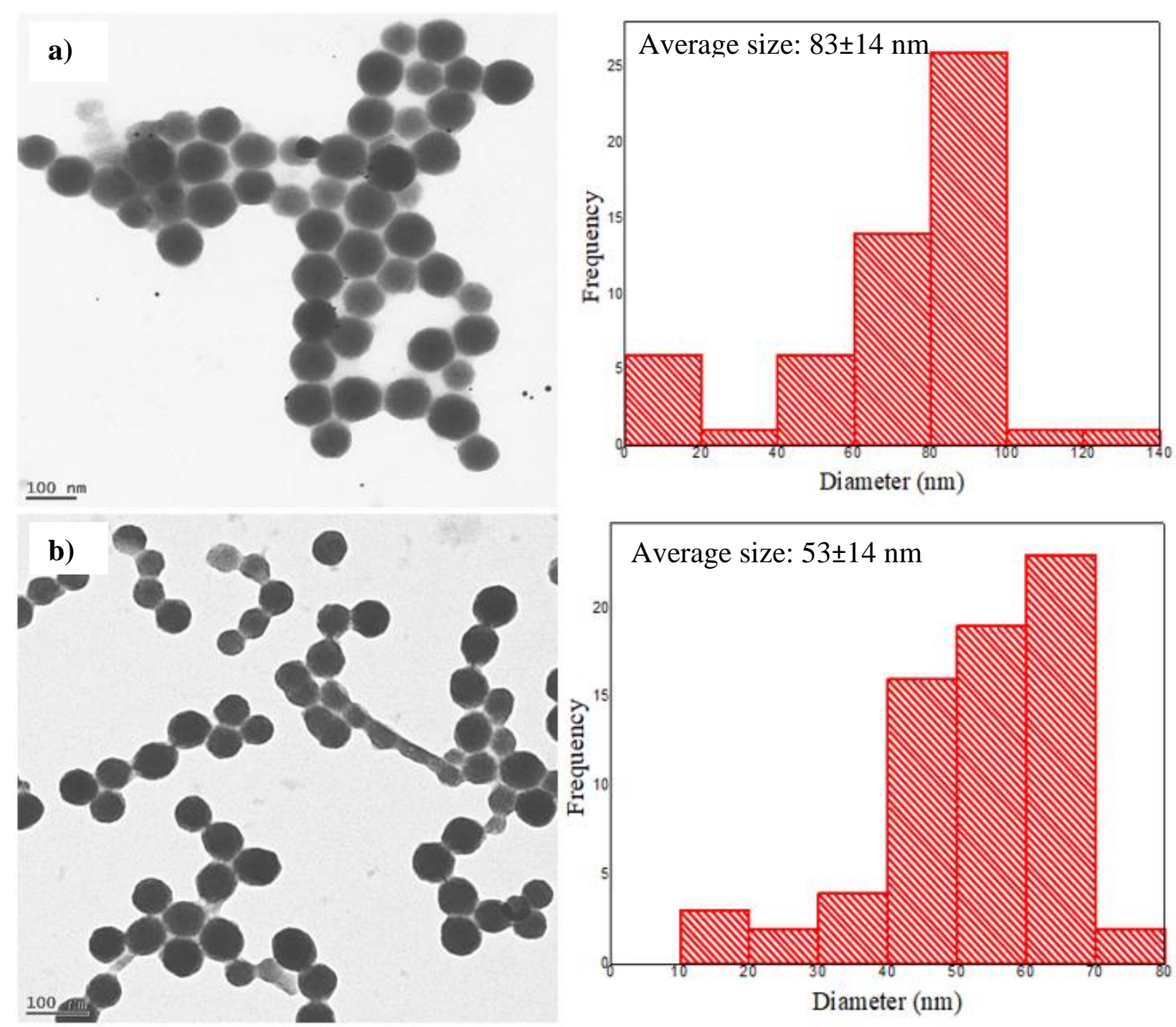

Figure 14: TEM image and size distribution of a) Res-PdNP-1 and b) Res-PdNP-2. 

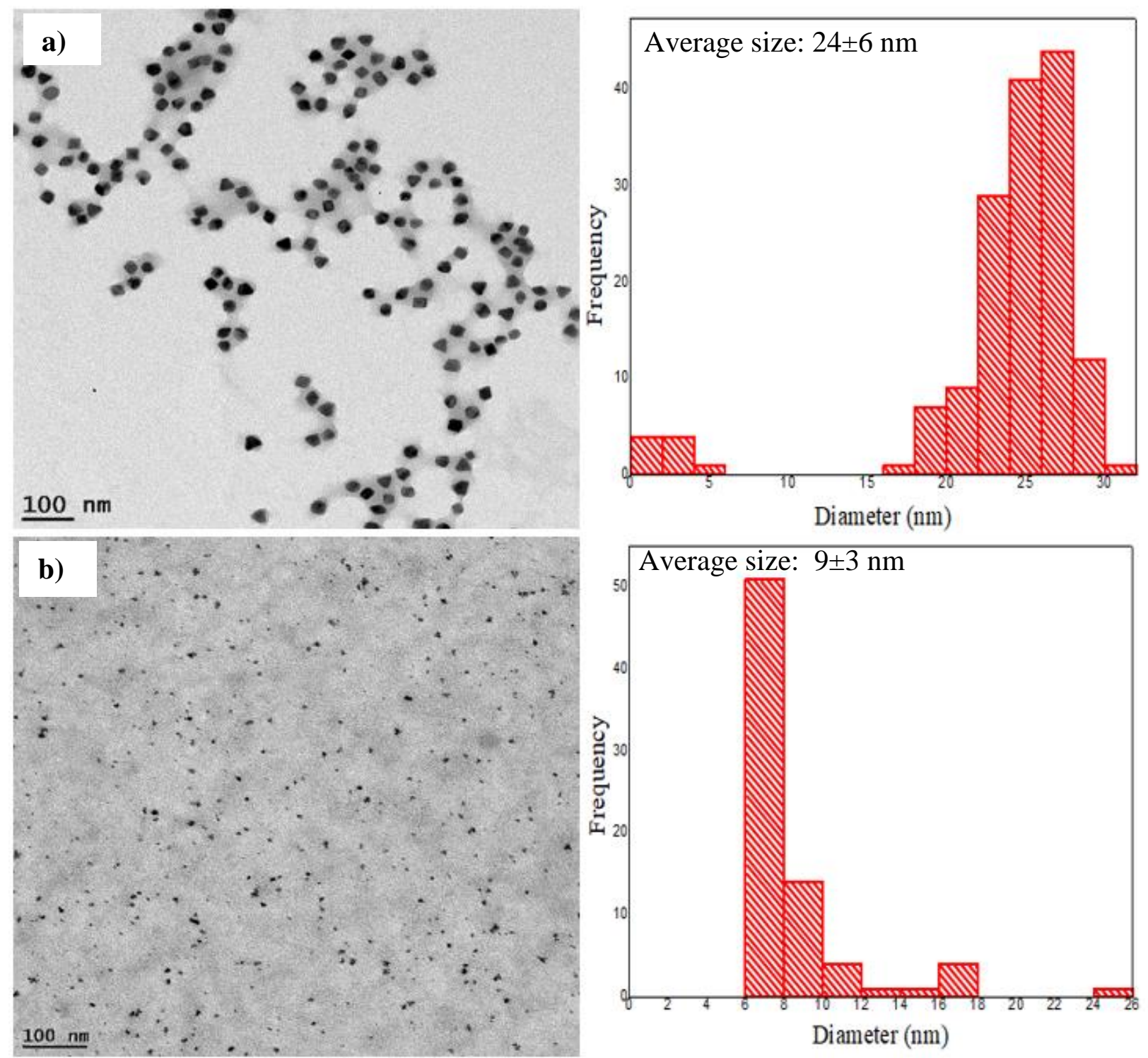

\section{Figure 15: TEM image and size distribution of a) Res-PdNP-3 and b) Res-PdNP-4.}

It is to be expected that the hydrodynamic sizes of PdNPs are significantly greater than the core sizes, confirming surface coating - that is Res-derived phenols and polyphenolic molecules have been encapsulated onto the surface of the palladium nanoparticles rendering them optimum in vitro and in vivo stabilities. Moreover, the surface coating of Res-PdNP-2 and Res-PdNP-4 is higher that Res-PdNP-1 and Res-PdNP-3 as a result of increased Res-derived phenols and polyphenols corona as shown in Table 6. Inductively coupled plasma - optical emission 
spectrometry (ICP-OES) was performed to estimate the palladium concentration in the ResPdNPs (Table 6).

Table 6: Physiochemical properties of Res-PdNPs

\begin{tabular}{|l|l|l|l|l|l|l|}
\hline Sample & $\begin{array}{c}\text { Hydrodynamic } \\
\text { size (nm) }\end{array}$ & $\begin{array}{c}\text { Core size } \\
(\mathbf{n m})\end{array}$ & $\begin{array}{c}\text { Surface } \\
\mathbf{c o a t i n g} \\
(\mathbf{n m})\end{array}$ & $\mathbf{P D I}$ & $\begin{array}{c}\text { Zeta } \\
(\mathbf{m V})\end{array}$ & \multicolumn{1}{|c|}{$\left[\begin{array}{c}\mathbf{P d}] \\
(\mathbf{\mu g} / \mathbf{m L})^{\mathbf{a}}\end{array}\right.$} \\
\hline Res-PdNP-1 & $93 \pm 4$ & $83 \pm 14$ & 10 & 0.3 & $-26 \pm 2$ & 165 \\
\hline Res-PdNP-2 & $254 \pm 18$ & $53 \pm 14$ & 201 & 0.5 & $-28 \pm 3$ & 166 \\
\hline Res-PdNP-3 & $44 \pm 1$ & $24 \pm 6$ & 21 & 0.3 & $-31 \pm 1$ & 167 \\
\hline Res-PdNP-4 & $117 \pm 4$ & $9 \pm 3$ & 108 & 0.3 & $-40 \pm 3$ & 167 \\
\hline
\end{tabular}

${ }^{a}$ ICP-OES, Geometric calculations revealed that [Pd] was expected at $177 \mu \mathrm{g} / \mathrm{mL}$

High-resolution TEM (HRTEM) analysis was performed to investigate crystallization, lattice planes and palladium atomic packing of the Res-PdNPs. The HRTEM images revealed that ResPdNP-1 and Res-PdNP-2 are amorphous nanoparticles that serve as intermediates for the generation of crystalline Pd nanoparticles; this was evident based on the results reported by Tianou et al. ${ }^{16}$ Moreover, the nucleation and crystallization of nanoparticles proceeds by two fundamental pathways i) aggregation of the Pd nanoclusters into larger assemblies formed by numerous nanoclusters $(0.5$ to $3 \mathrm{~nm})$ and ii) structural rearrangement with pre-nucleation $\mathrm{Pd}$ nanoclusters towards direct conversion to crystalline nanoparticles as shown in Figure $16 .{ }^{17}$ 

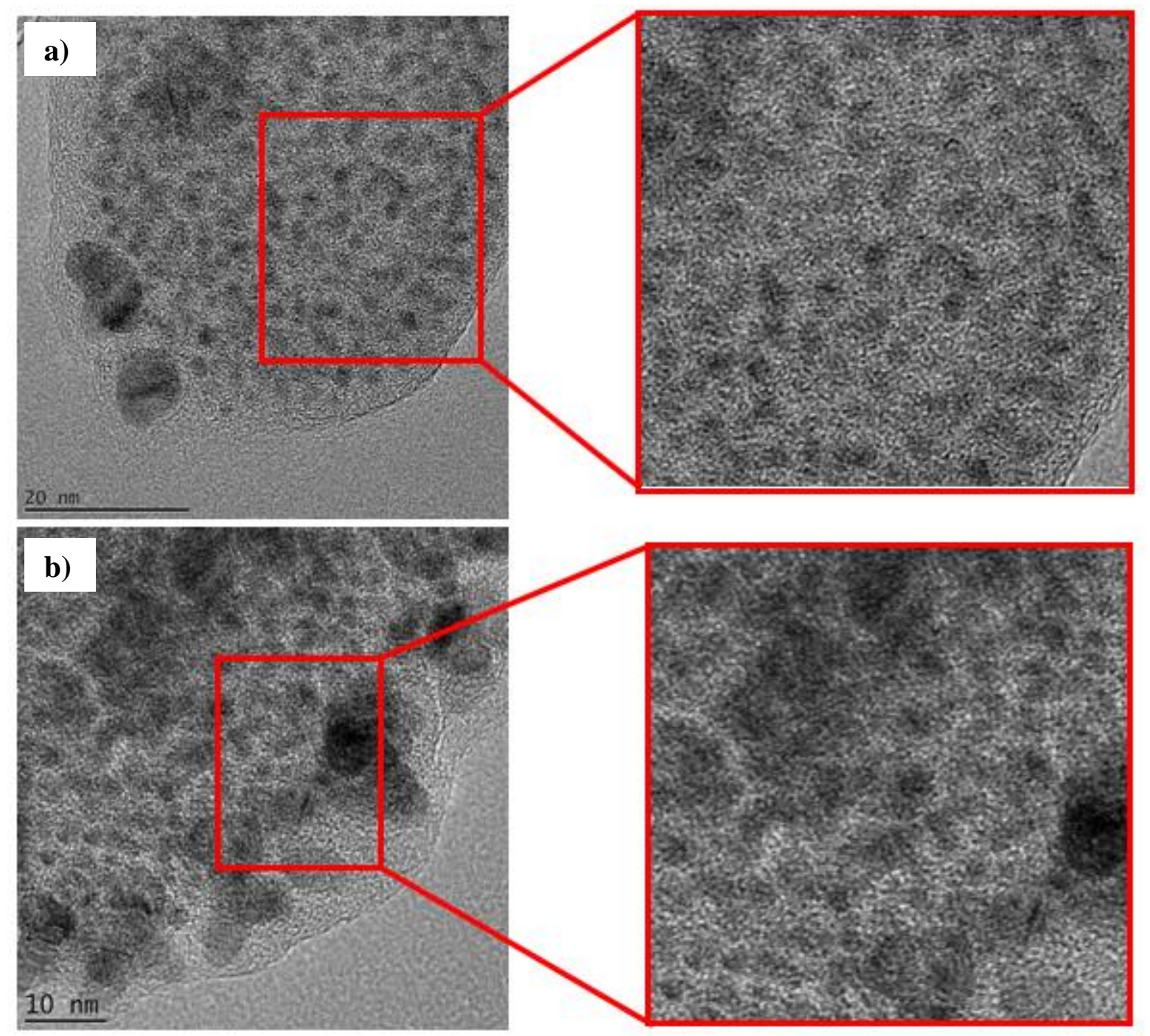

c)

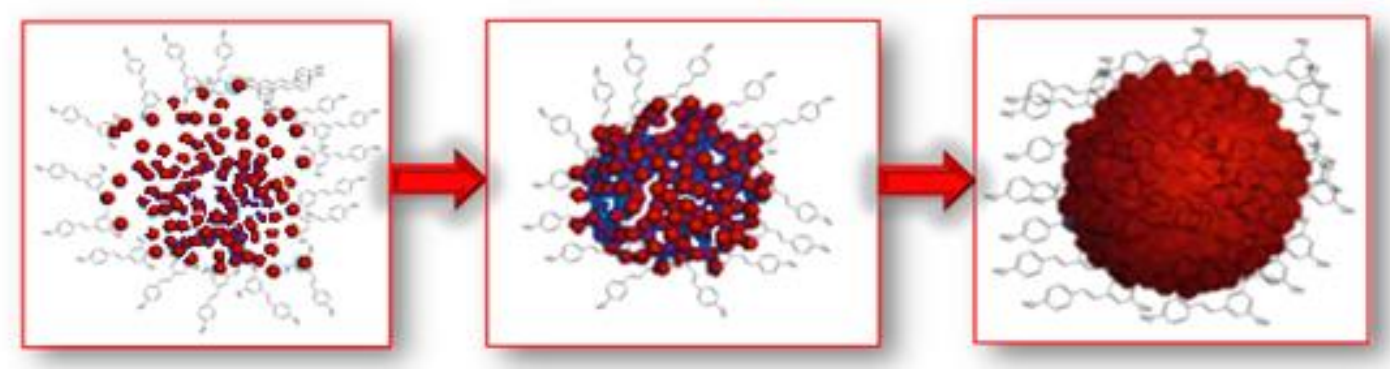

Figure 16: HRTEM images of a) Res-PdNP-1, b) Res-PdNP-2 confirming the existence of individual Pd nanoclusters and c) schematic drawing visualizing Pd nanocluster agglomeration (structures are not drawn to scale). 
The addition of GA allowed for the production of thermodynamically stable nanoparticles through kinetically inhibiting the rate of nucleation via direct crystallization. This resulted in particles with a higher stability; furthermore, the HRTEM images of Res-PdNP-3 and ResPdNP-4 revealed that the nanoparticles are crystalline with interplanar lattice d-spacing of 0.2 $\mathrm{nm}$ between the lattice planes (Figure 17). ${ }^{14}$ 

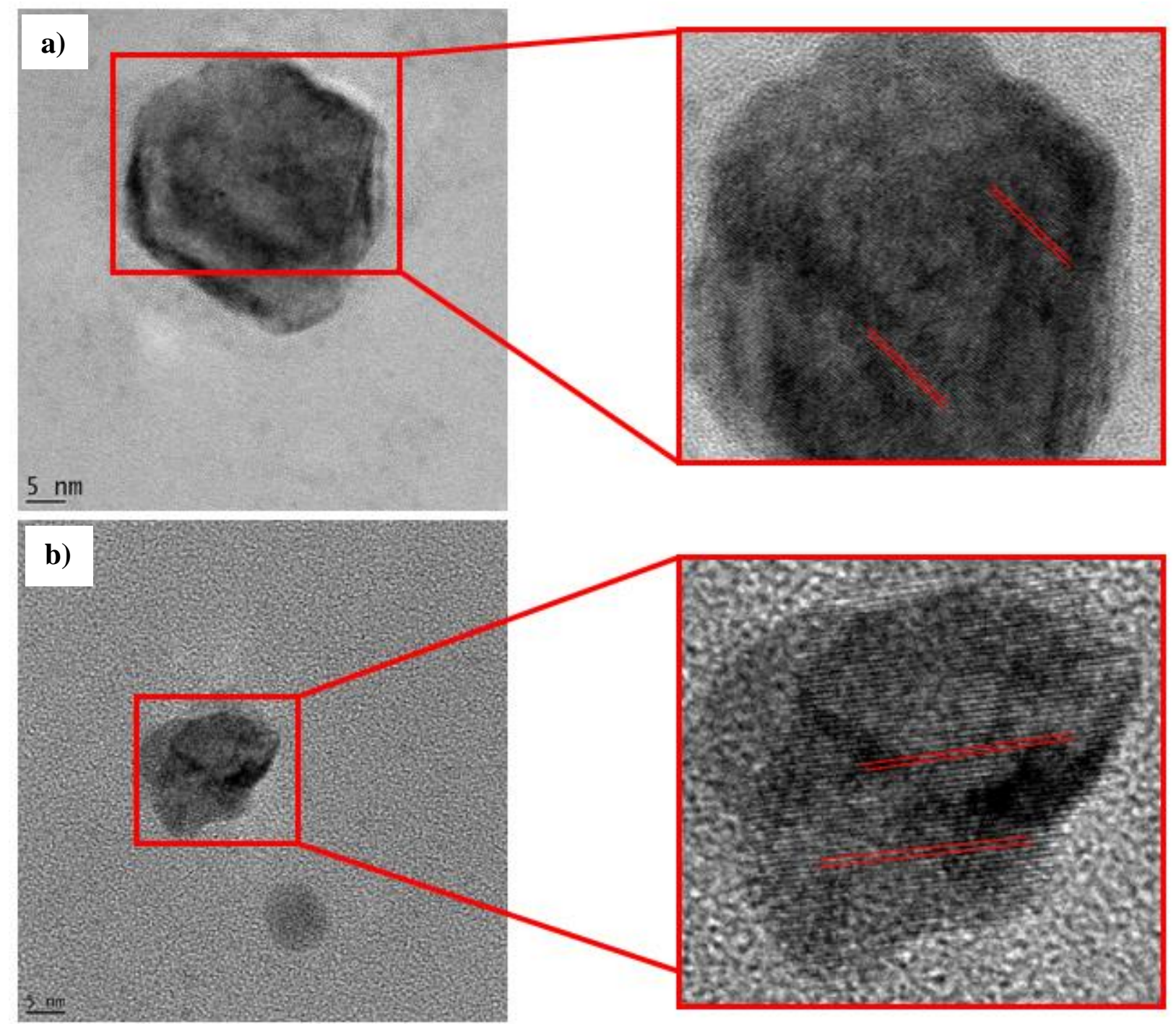

c)

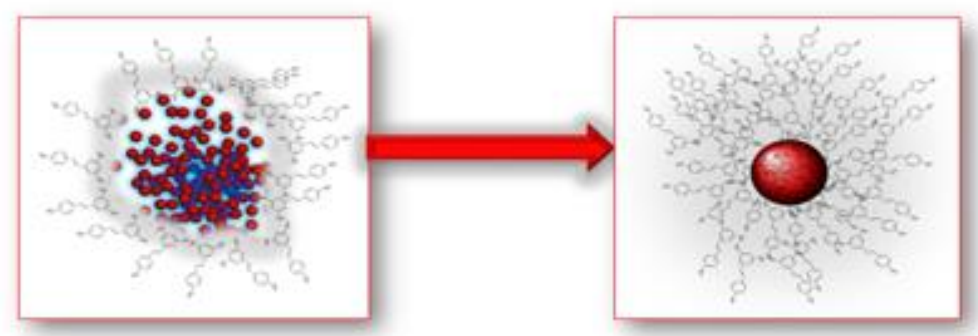

Figure 17: HRTEM images of a) Res-PdNP-3, b) Res-PdNP-4 crystalline nature and c) Schematic drawing visualizing PdNPs nanocrystals (structures are not drawn to scale). 
The effect of GA is to facilitate the interface transition from an amorphous to a crystalline resveratrol conjugated PdNPs. ${ }^{18}$ Thanh et al. ${ }^{19}$ demonstrated the nucleation and growth of nanoparticles in solution. They reported that during nucleation growth, small nanoparticulates coalescence through surface and strain energies (energy stored in a system that allows for atomic rearrangement) leading to the formation of an interface. These energies of interface between the crystalline nucleus of the small Pd nanoparticulates and the amorphous PdNPs intermediates will be larger than the activation energy, thereby allowing atomic rearrangements triggered by the non-Newtonian shear-thinning behavior of GA in solution to propagate crystallization waves through anchoring and compacting the amorphous nanoparticles, leading to mono- or polycrystalline face-centered cubic (FCC) structures. ${ }^{18,20-22}$ Figure 18 shows a schematic illustration of a crystallization mechanism of amorphous-crystalline interface of PdNPs, crystallization is driven by long-range elastic interaction due to small crystal nucleus formation in amorphous nanoparticles is induced by short-range atomic rearrangements. ${ }^{23}$ 


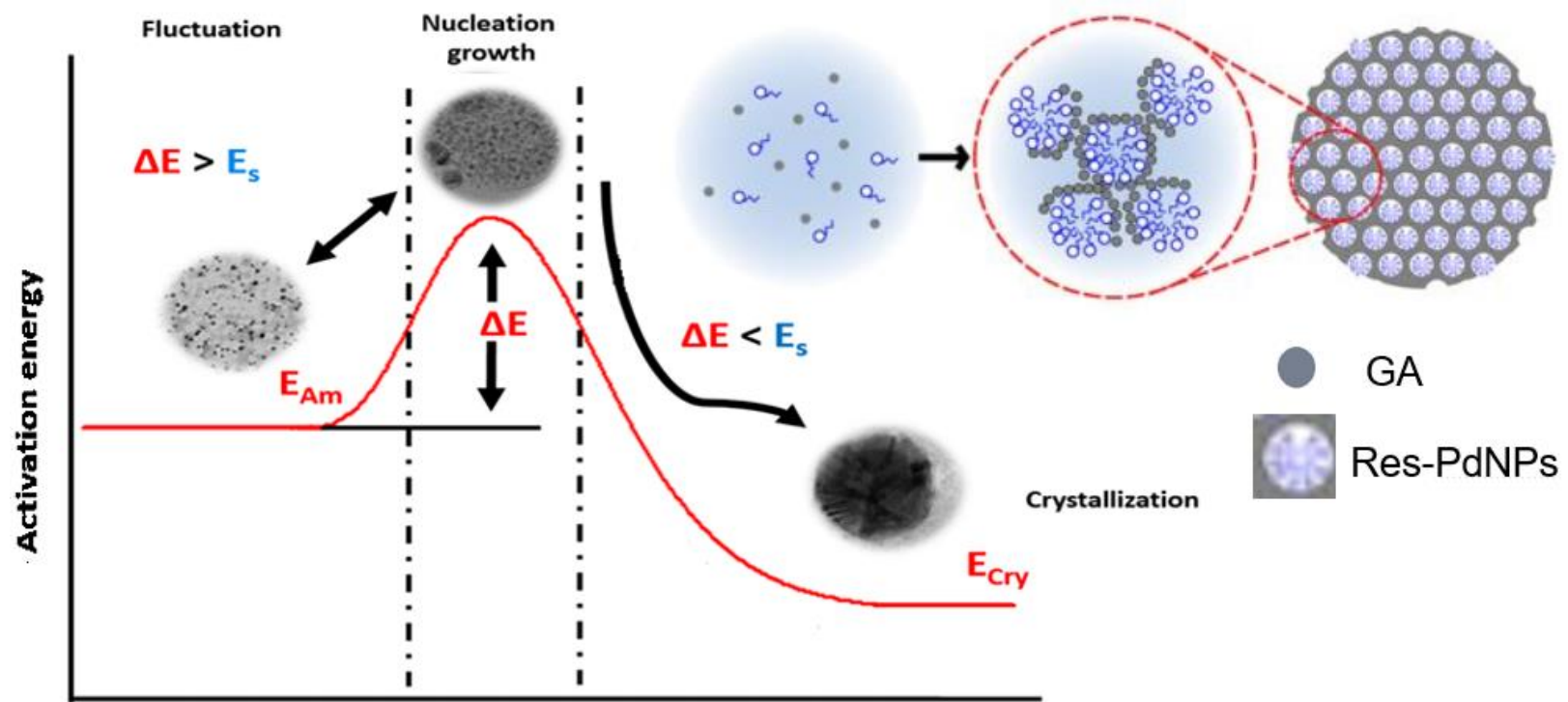

Reaction coordinate

$E_{s}$ - Strain energy of amorphous PdNPs intermediates $\Delta \mathrm{E}$ - Activation energy

Figure 18: Crystal growth of palladium nanoparticles influenced by GA, and a schematic illustration of crystallization mechanism of amorphous palladium nanoparticles. ${ }^{19}$

\subsection{Elemental analysis of Res-PdNPs}

The scanning transmission electron microscope (STEM)-high-angle annular dark-field (HAADF) imaging and energy-dispersive X-ray spectroscopy (EDS) mapping allowed for further confirmation for the presence of elemental Pd. All the Res-PdNPs samples showed the presence of $\mathrm{Pd}$ as shown in Figure 19A and 19B. The presence of $\mathrm{Cu}$ peaks is from the $\mathrm{Cu}$ grid and the $\mathrm{Na}$ and $\mathrm{Cl}$ are by-products from the reaction during the synthesis of Res-PdNPs. 


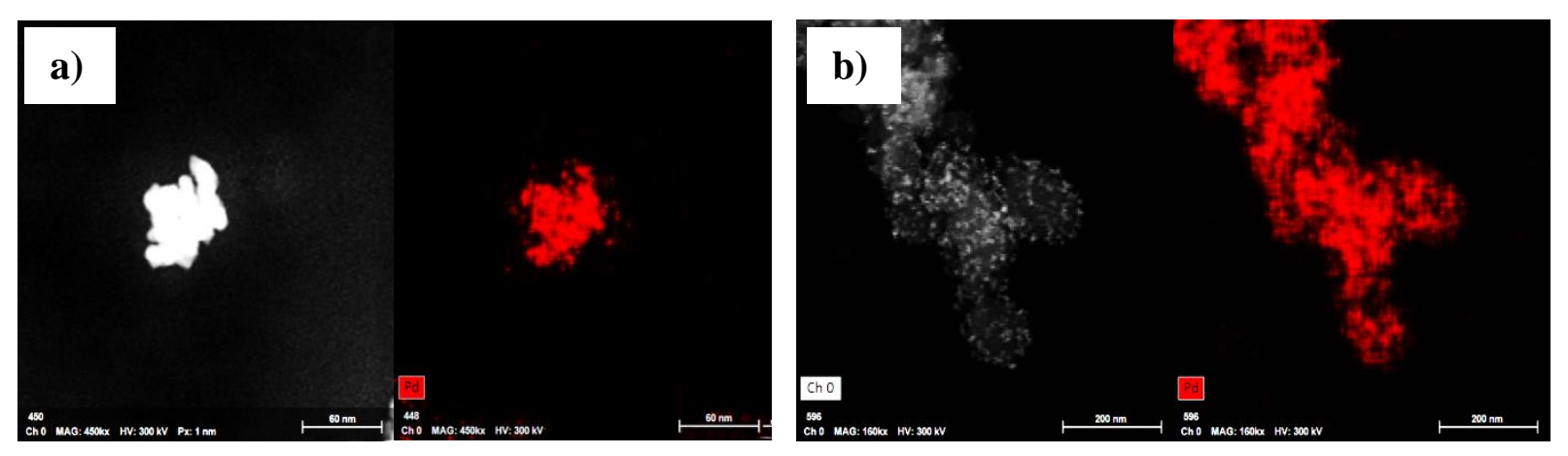

c)

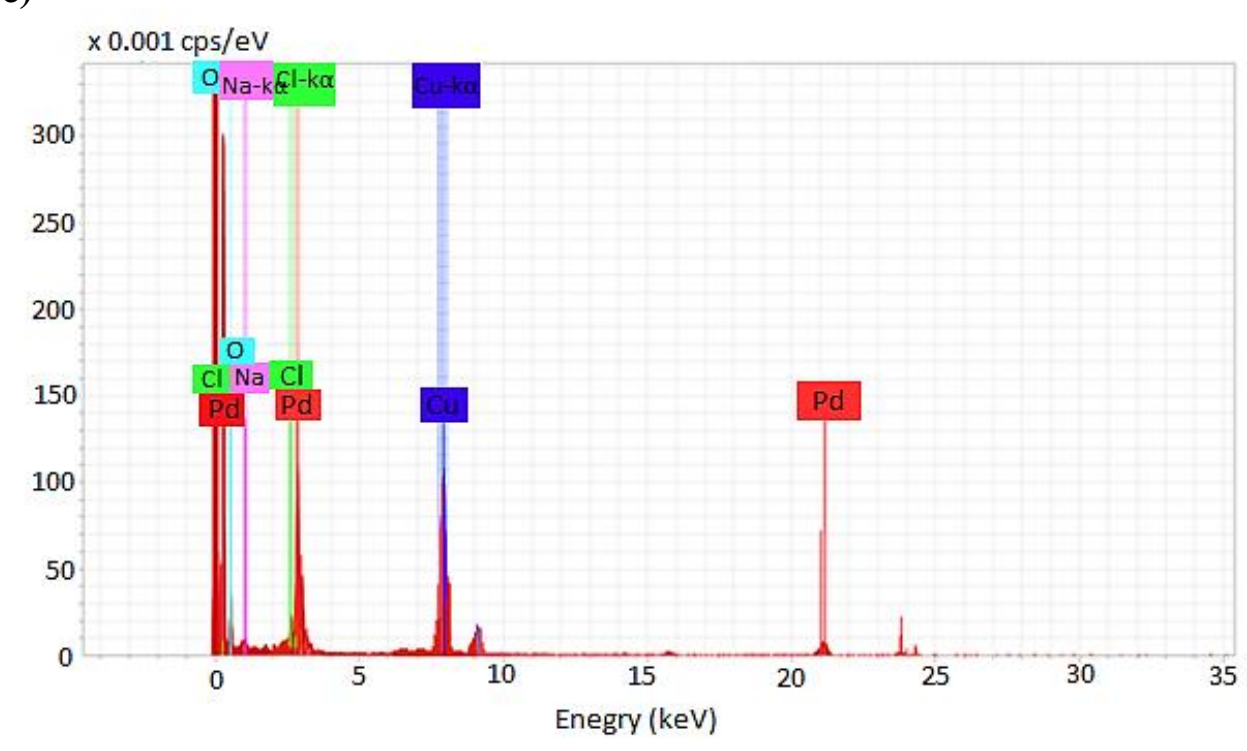

Figure 19A: STEM-HAADF image and EDS elemental maps showing the presence of Pd, $\mathrm{O}, \mathrm{Cu}, \mathrm{Na}$ and $\mathrm{Cl}$ in a) Res-PdNP-1, b) Res-PdNP-2, respectively and c) EDS spectrum of Res-PdNP-2.

The EDS mapping of Res-PdNP-4 revealed a spatial distribution of Pd as shown in Figure 19B, that further corroborates the anchoring and compacting capacity of PdNPs facilitated by GA supportive matrix. 

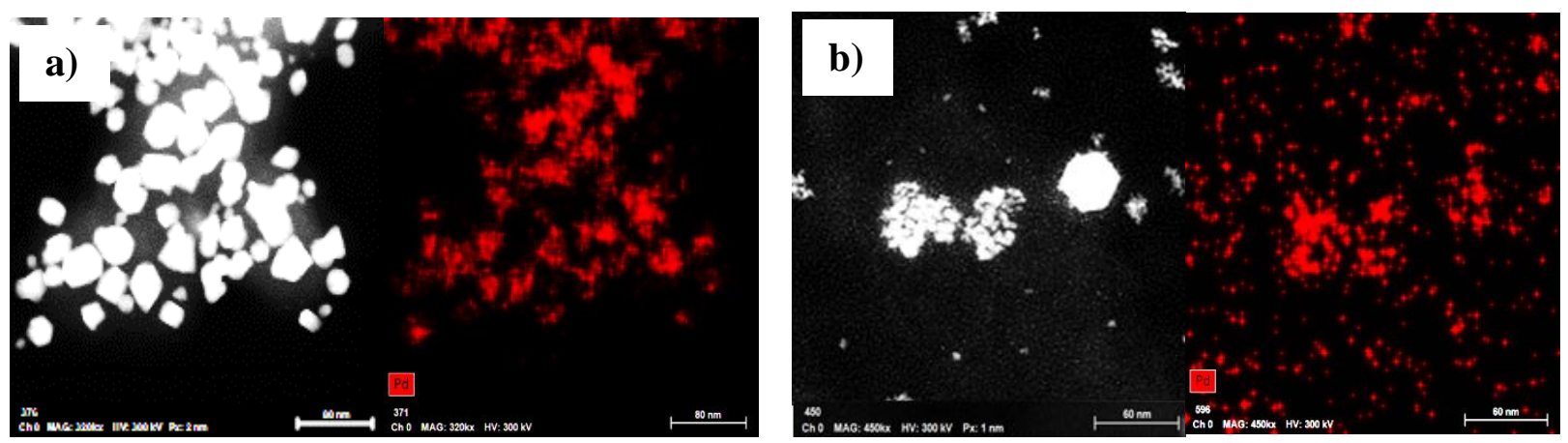

c)

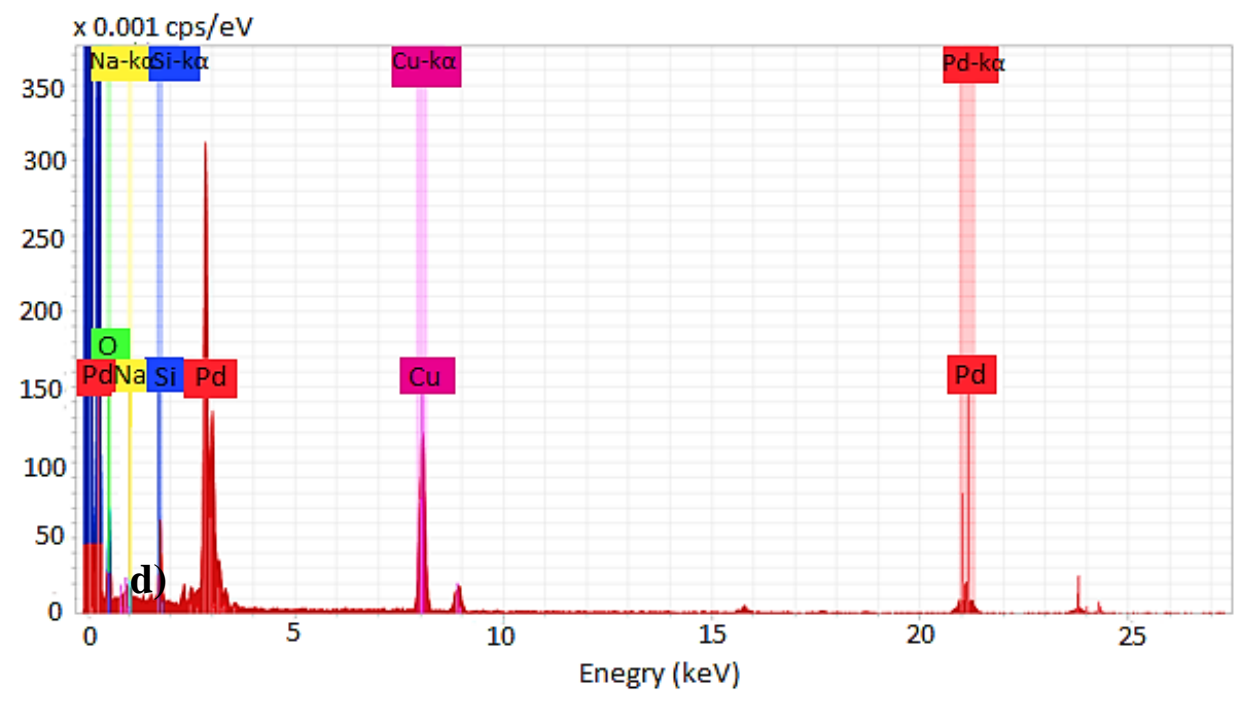

Figure 19B: STEM-HAADF image and EDS elemental maps showing the presence of Pd, O, Cu, Si, Na and Cl in a) Res-PdNP-3, b) Res-PdNP-4 and c) EDS spectrum of Res-PdNP4.

\subsection{In vitro stability of palladium nanoparticles}

The stability of the Res-PdNPs was evaluated by monitoring the size of the nanoparticles in various biological media $(1 \% \mathrm{NaCl}, 0.5 \%$ cysteine, $0.2 \mathrm{M}$ histidine, $0.5 \%$ human serum albumin (HSA), $0.5 \%$ bovine serum albumin (BSA), and $\mathrm{pH} 5, \mathrm{pH} 7$, and $\mathrm{pH} 9$ at $\left.37^{\circ} \mathrm{C}\right)$ in triples $(\mathrm{n}=3)$. Moreover, the Res-PdNPs size was also monitored in a mixture of the above-mentioned biological media at $37{ }^{\circ} \mathrm{C}$ to mimic in vivo conditions for a week. The results showed that the 
size of the Res-PdNP-1 and Res-PdNP-2 were not stable in bovine serum albumin (BSA), human serum albumin (HSA), and the mixture solution. The Res-PdNP-3 and Res-PdNP-4 exhibited increased stability with no tendency to aggregate, thereby demonstrating superior in vitro stability in the tested biological fluids. This was attributed to the presence of GA, which confers high cohesive and adhesive properties towards improved stability of Res-PdNPs (Figure 20). ${ }^{24}$ Several studies by Gamal-Eldeen et al. ${ }^{25,26}$, Fent et al. ${ }^{27}$ de Barros et al. ${ }^{28}$, Wu et al. ${ }^{29}$, Williams et al. $^{30}$ demonstrated that the addition of GA promoted colloidal stabilization, which was primarily attributed to the existence of steric forces from the GA that prevents the aggregation of nanoparticles. Based on the stability, only Res-PdNP-3 and Res-PdNP-4 were investigated further due to their superior stability. 


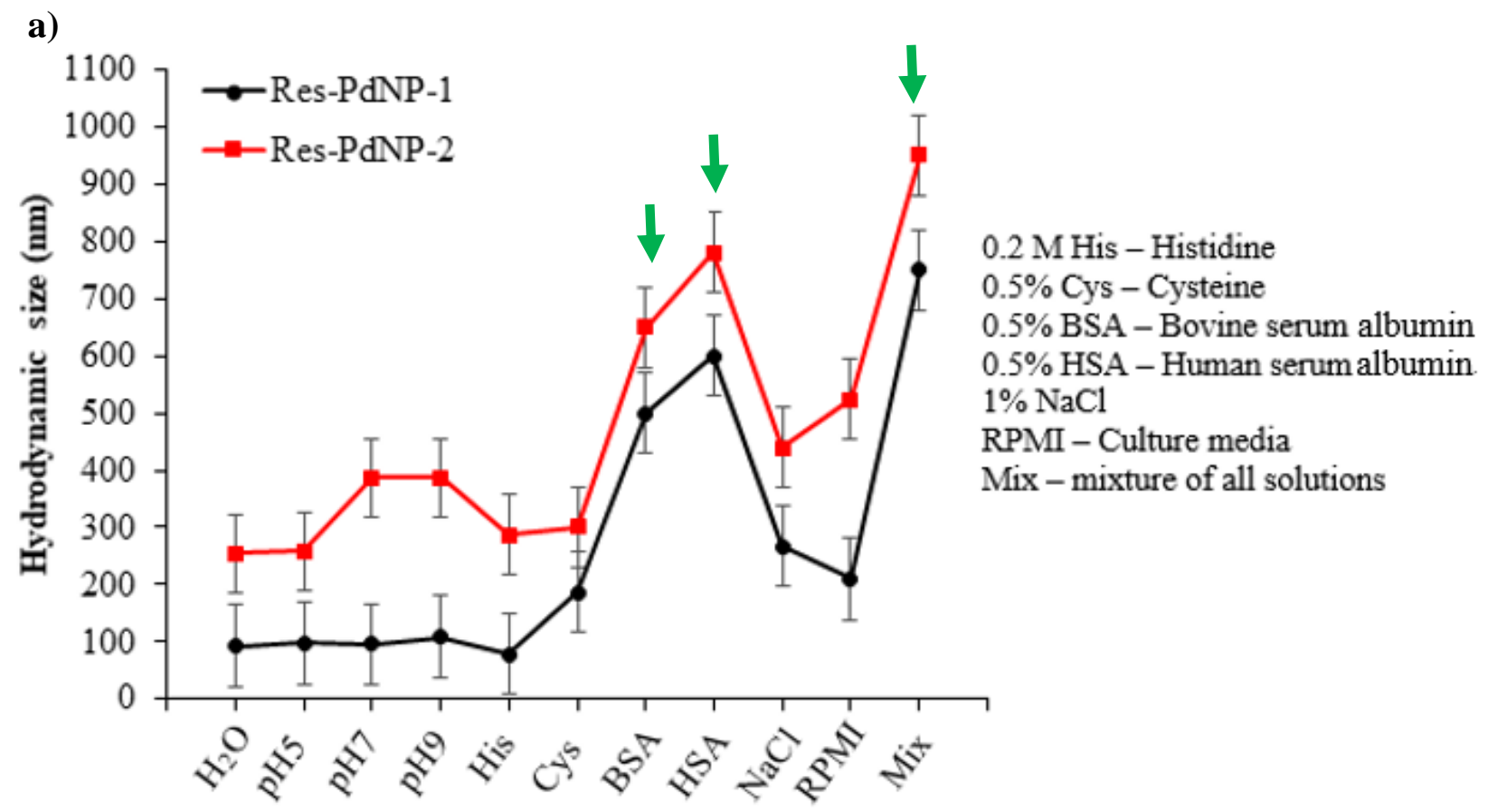

Biological media

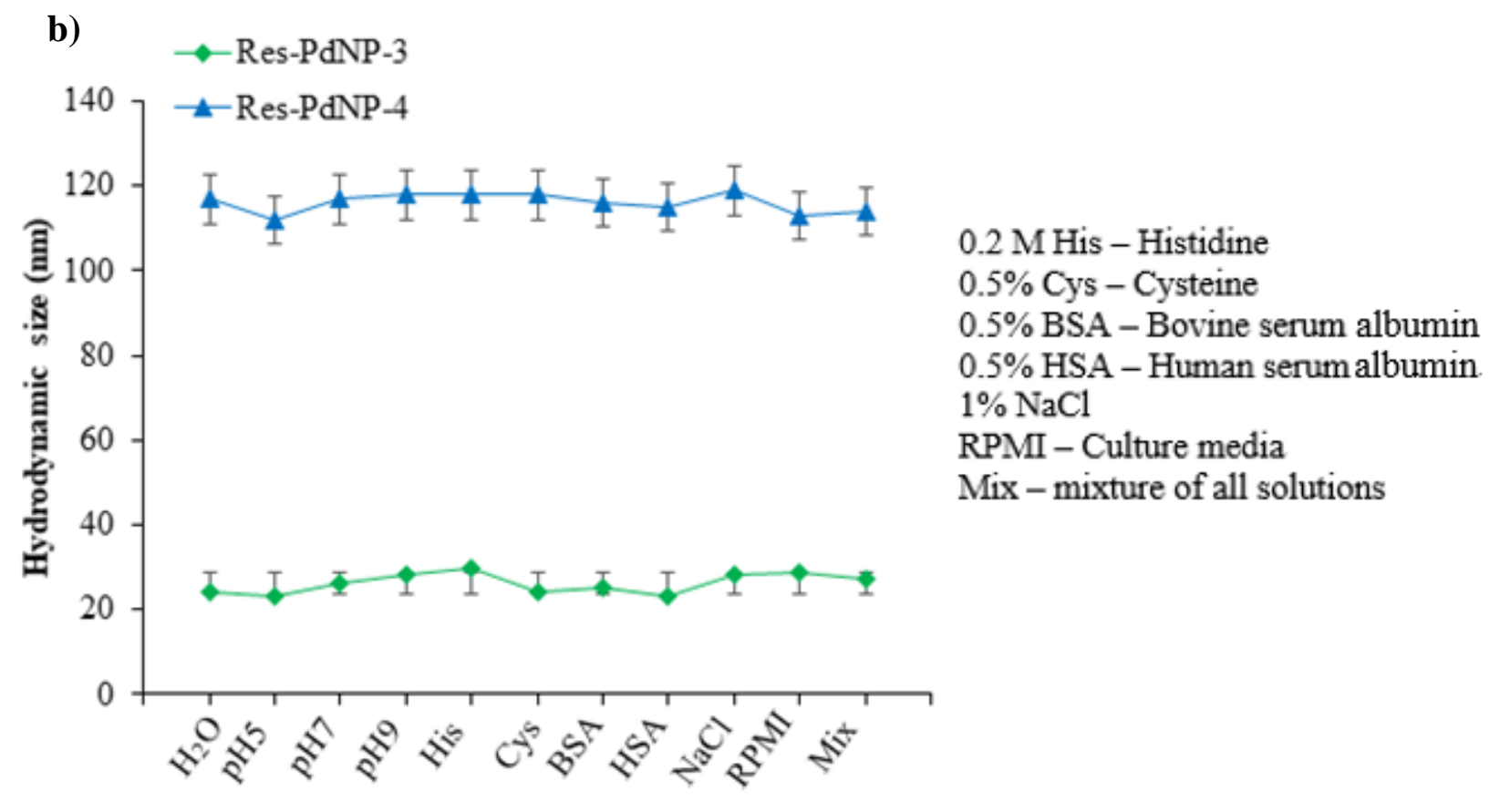

Biological media

Figure 20: In vitro stability assay of Res-PdNPs by monitoring the hydrodynamic size of a) Res-PdNP-1 and Res-PdNP-2 and b) Res-PdNP-3 and Res-PdNP-4 in various biological media at $37^{\circ} \mathrm{C}$ for a week $(\mathrm{n}=3)$. Res-PdNP-1 and Res-PdNP-2 not stable in media (green arrow) 


\subsection{Fourier transform-infrared (FTIR) of Res-PdNP-3 and Res-PdNP-4}

The functional group conjugation of Res-derived phenols and polyphenols on the surface of PdNPs was investigated by FTIR spectroscopy. An intensive wide band emerged in the range

$3363-3388 \mathrm{~cm}^{-1}$, attributed to the presence of phenolic hydroxyl groups (O-H stretching) of Res, the broad range is due to the formation of extensive hydrogen bonding among polyphenolic molecules in solution. ${ }^{31}$ The band at $2114-2150 \mathrm{~cm}^{-1}$ is attributed to the $-\mathrm{C} \equiv \mathrm{C}$ - stretching vibrations, which can be attributed to the oxidative products of Res (triple-bond resveratrol analog) and the peak at $1641-1645 \mathrm{~cm}^{-1}$ was attributed to the stretching vibration of $-\mathrm{C}=\mathrm{C}$ between the aromatic rings. The bands at $951-982 \mathrm{~cm}^{-1}$ were attributed to the in-plane bending vibration of $=\mathrm{C}-\mathrm{H}$ in the aromatic ring, however this band shifted to $890 \mathrm{~cm}^{-1}$ reflecting out-ofplane bending of the exomethylene groups in Res-PdNP-3. The peak at 573-640 $\mathrm{cm}^{-1}$ of free Res and Res-PdNP-4 is from monosubstitution (phenol) C-H bends. These results indicate that Res molecules were conjugated to the Res-PdNPs surface with high affinity for the metal center and generally does not dissociate under physiological conditions as indicated by high stability (Figure 21). 


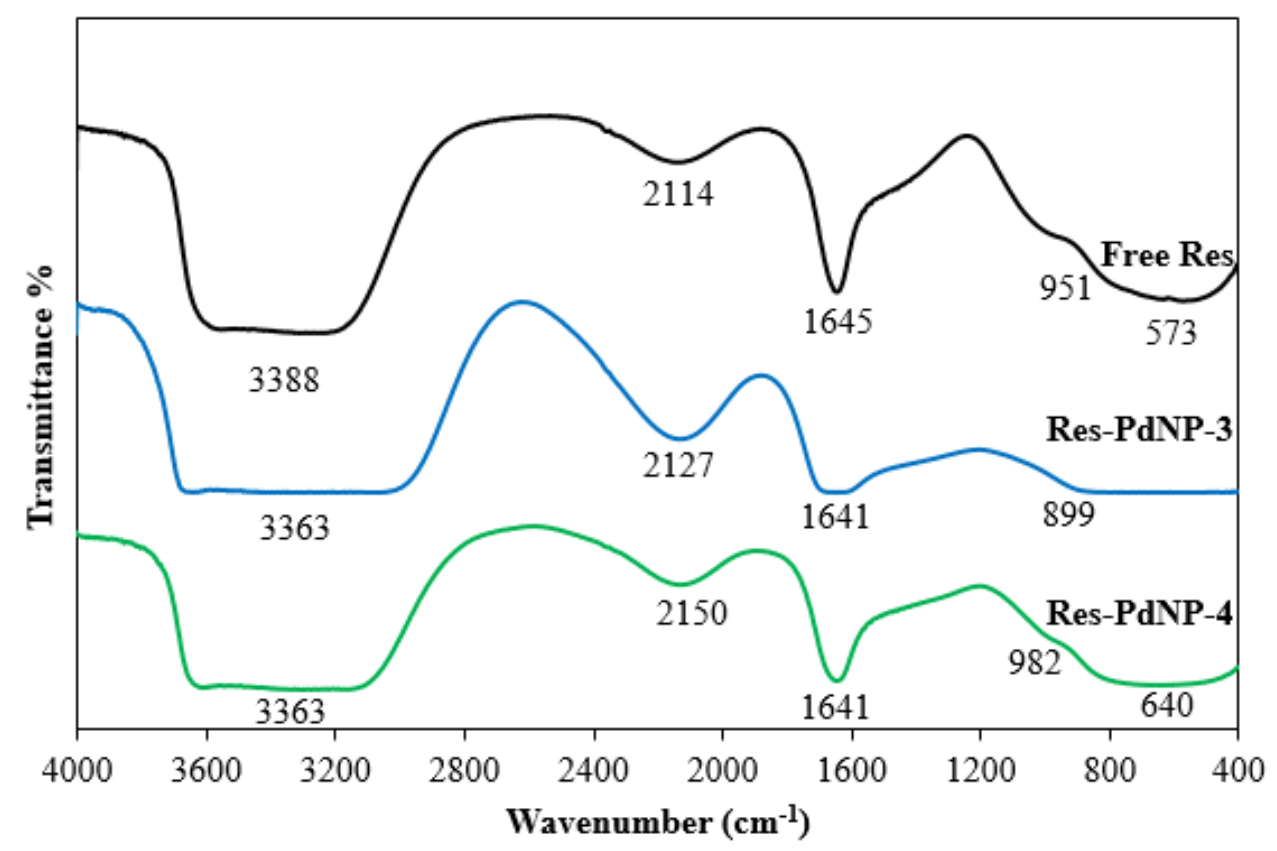

Figure 21: Fourier transform-infrared (FTIR) spectra of free Res and Res-PdNPs.

\subsection{Quantification of Res-derived phenols and polyphenolic molecules encapsulated onto} the surface of the nanoparticles by liquid chromatography-tandem mass spectrometry with multiple reaction monitoring (LC-MS/MRM)

The concentration of Res-derived phenols and polyphenolic molecules encapsulated on the surface of Res-PdNP-3 and Res-PdNP-4 was determined to be 6 and $20 \mu \mathrm{g} / \mathrm{mL}$, respectively as quantified using LC-MS/MS with multiple reaction monitoring (MRM) on the Quantiva (Figure 22). Res-PdNP-3 significantly had lower Res compared to free Res because some of the Res were oxidized in the reaction for the reduction of $\mathrm{Pd}^{(\mathrm{II})}$ ions to produce Res-PdNPs and subsequently capping the nanoparticles. On the other hand, Res-PdNP-4 had a higher concentration of loaded Res-derived phenols and polyphenols attributed to the increased Resderived phenols and polyphenols corona on the Re-PdNP-4, thereby corroborating with the data obtained from DLS. 

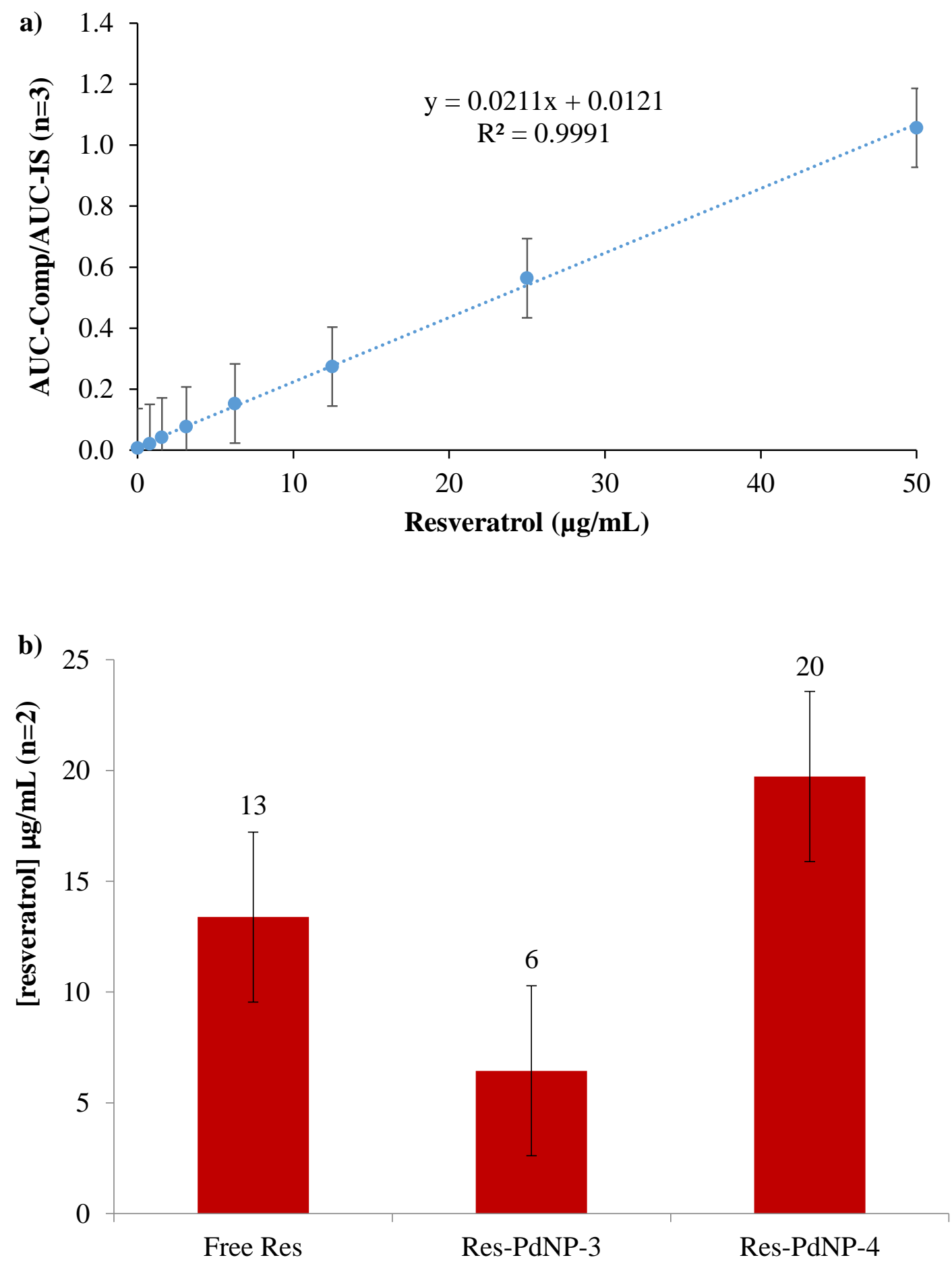

Figure 22: LC-MS/MRM Quantitation of Res a) Res standard curve normalized to AUC for tolbutamide internal standard and b) Res-PdNPs monitored by MRM to specifically quantify the resveratrol in the samples. 


\section{7 ${ }^{1} \mathrm{H}$ nuclear magnetic resonance (NMR) analysis of the Res-PdNP-4}

The presence of Res-derived phenols and polyphenols encapsulated on the Res-PdNP-4 was investigated by ${ }^{1} \mathrm{H}$ NMR. Figure 23 shows the ${ }^{1} \mathrm{H}$ NMR spectrum Res, GA and Res-GA mixture. The characteristic peaks of Res are seen in the region between chemical shifts $(\delta) 6-8$ ppm and the crowded signals between $\delta 3-5.5 \mathrm{ppm}$, which are characteristic signals of polysaccharides $\left(\mathrm{OH}\right.$ and $\mathrm{CH}$ group of mannose $(\delta 3.65-3.60 \mathrm{ppm}), \mathrm{CH}_{2}$ group of arabinose $(\delta 3.81-3.55$ ppm), $\mathrm{C}_{4}$ of galactose $(\delta 3.98,4.28 \mathrm{ppm}), \beta$-sugar residues $(\delta 4.92-4.96 \mathrm{ppm})$ and $\alpha$-sugar residues $(\delta 5.1-5.3 \mathrm{ppm}))$, while the upfield peak at $1.4 \mathrm{ppm}$ is assigned to the methyl group of the rhamnose sugar of GA (Figure 23a and b, respectively). ${ }^{32-38}$ The ${ }^{1} \mathrm{H}$ NMR spectrum of the Res-GA mixture was collected, as shown in Figure 23c, and revealed that the Res spectra does not overlap the GA spectra, thus allowing clear distinction and identification. 


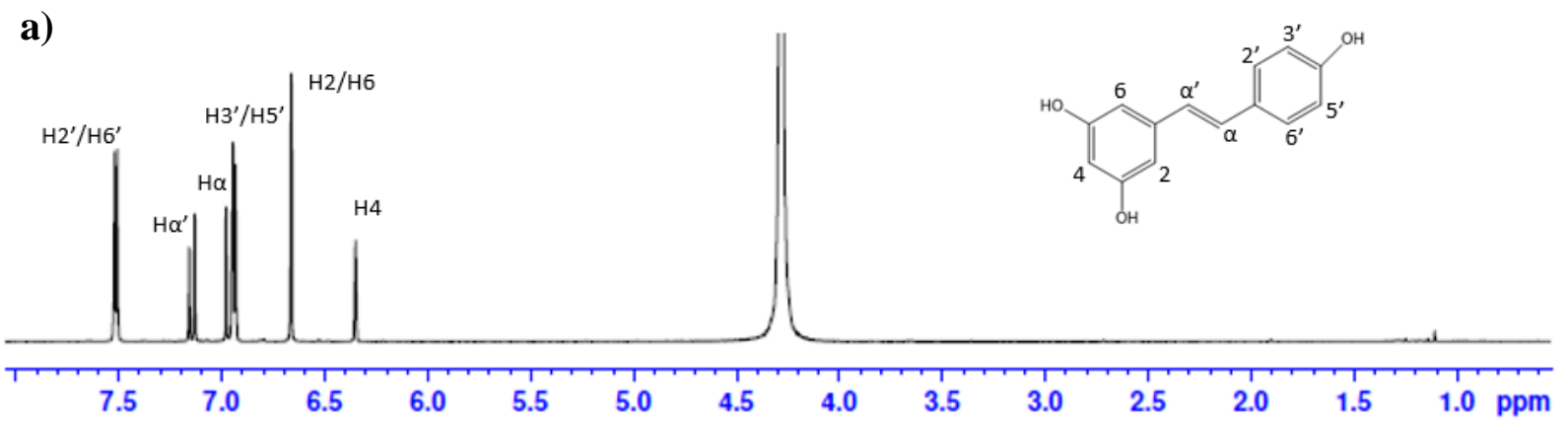

b)

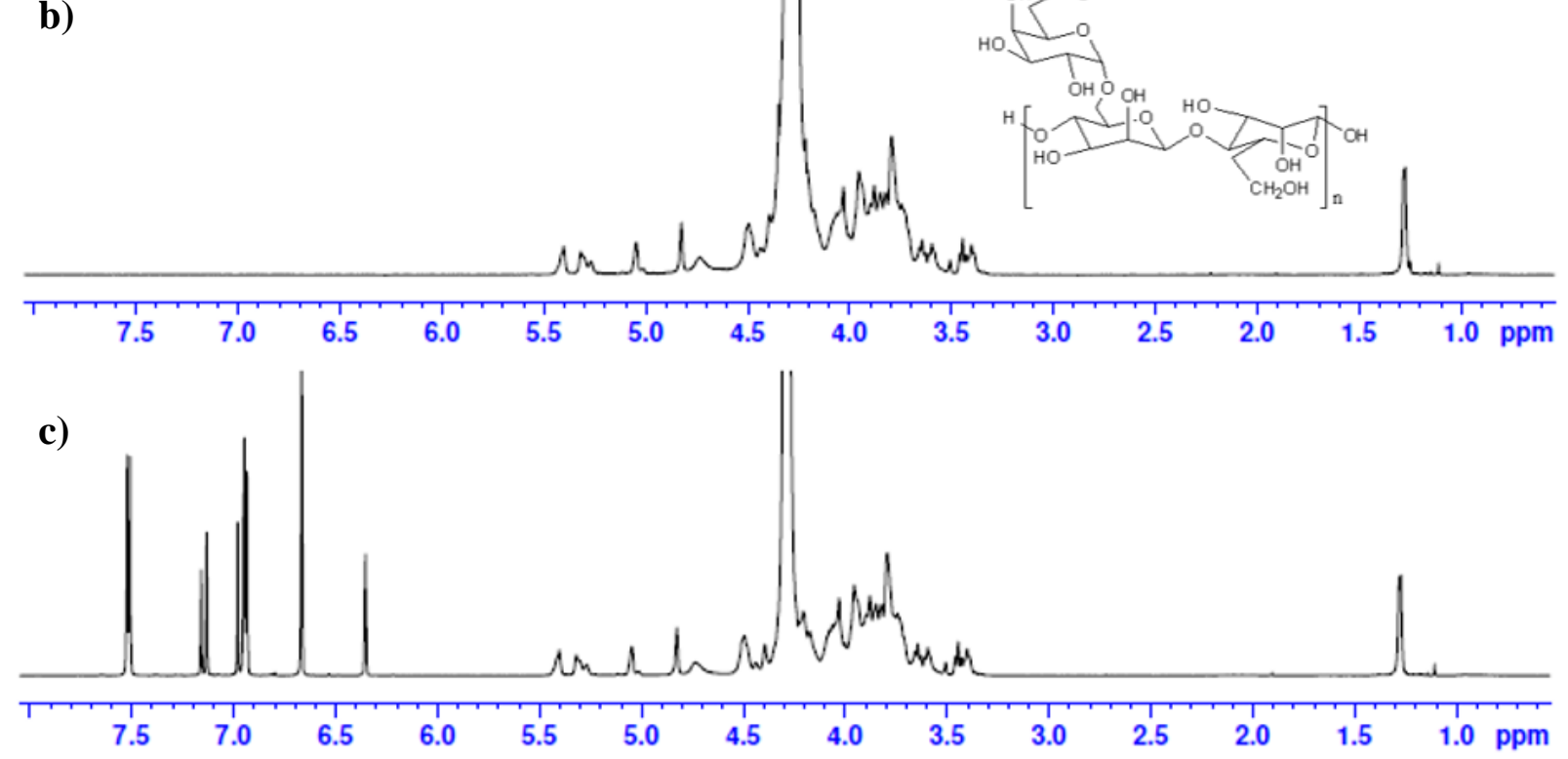

\section{Figure 23: ${ }^{1} \mathrm{H}$ NMR spectra of a) Free Res, b) GA and c) Res-GA mixture in $\mathrm{D}_{2} \mathrm{O}$.}

The ${ }^{1} \mathrm{H}$ NMR spectra of Res-PdNP-4 was different to the spectra of Res-GA mixture as shown in Figure 24. The intensity of the peaks corresponding to Res is less and significantly different in the Res-PdNP-4 compared to that observed in the spectrum of free Res. Notably, this further corroborates that Pd(II) was reduced to PdNPs via the oxidation of Res, while GA shows slight changes but mostly looks the same. ${ }^{39}$ Figure 24 insert had high signal to noise with limited integration details obtained. Hence understanding the oxidation product is tricky. This result reveals the presence of Res-derived phenols and polyphenols due to the oxidation of Res. The GA shows slight changes but mostly looks the same to me. 


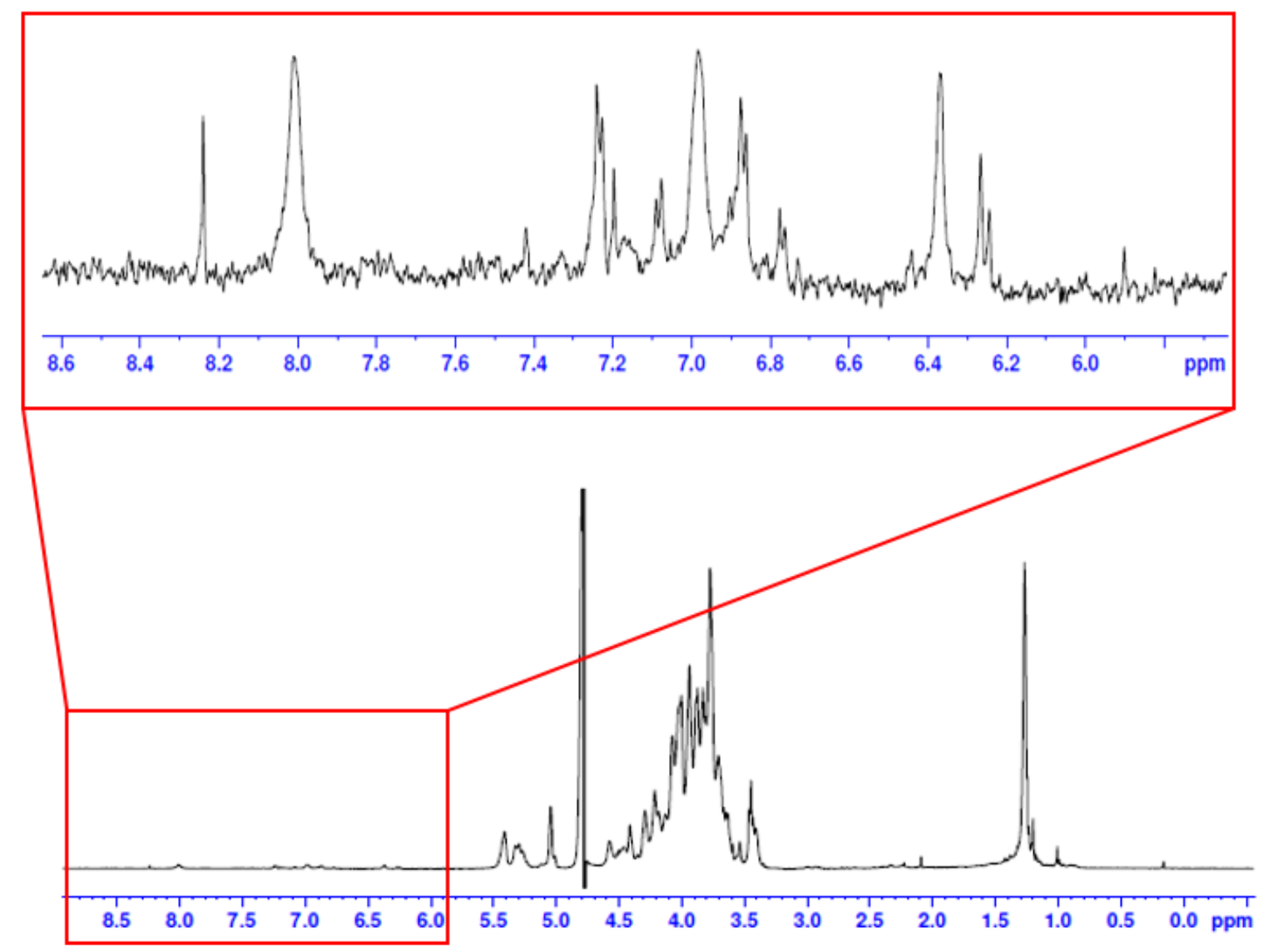

Figure 24: ${ }^{1} \mathrm{H}$ NMR spectra of Res-PdNP-4 in $\mathrm{D}_{2} \mathrm{O}$ (insert: magnified Res region).

A study by Camont et al. ${ }^{40}$ reported that $\sim 85 \%$ of oxidization end-products of trans-Res are piceatannol and 3,5-dihydroxybenzoic acid, while $\sim 15 \%$ is attributed to 3,5dihydroxybenzaldehyde and para-hydroxybenzaldehyde. The structure of these oxidation endproducts of trans-Res are listed in Figure 25. 
(a)<smiles>Oc1ccc(/C=C/c2cc(O)cc(O)c2)cc1</smiles>

Resveratrol

(c)<smiles>O=C(O)c1cc(O)cc(O)c1</smiles>

3,5-dihydroxybenzoic acid (b)<smiles>Oc1cc(O)cc(/C=C/c2ccc(O)c(O)c2)c1</smiles>

Piceatannol

(e)<smiles>O=Cc1ccc(O)cc1</smiles>

Figure 25: Chemical structure of resveratrol (a) and of its oxidation products piceatannol (b), 3,5-dihydroxybenzoic acid (c), 3,5-dihydroxybenzaldehyde (d) and parahydroxybenzaldehyde (e). ${ }^{40}$

There are several mechanisms that can explain the ${ }^{1} \mathrm{H}$ NMR spectra during the synthesis of PdNPs using Res. The reduction reaction follows an electron transfer mechanism that involves the release of both electrons and protons from the Res molecule. ${ }^{41}$ Based on the quantum chemistry calculations by the Functional of Density Theory method for the determination of antioxidant pharmacophore of Res. Res contains both resorcinol and phenol moieties and the B ring of resveratrol is more easily oxidized compared to the resorcinol ring A due to its lower ionization potential. ${ }^{41-43}$ During the production of Res-PdNPs, $\mathrm{Pd}^{2+}$ is reduced to $\mathrm{Pd}^{0}$ by an electron transfer facilitated by Res chelating complex with $\mathrm{Pd}^{2+}$ ion resulting in $\mathrm{Res}$ oxidizing to quinone which can be expressed stoichiometrically as Eq. 7 and Figure 26:

$$
\mathrm{nPd}^{2+}+2 \mathrm{R}-(\mathrm{OH}) \mathrm{n} \rightarrow \mathrm{nPd}^{0}+2 \mathrm{nR}=\mathrm{O}+2 \mathrm{nH}^{+}
$$


Where $\mathrm{R}$ and $\mathrm{n}$ represent the heterocyclic or alkyl groups and the number of the hydroxyl groups oxidised by $\mathrm{Pd}(\mathrm{II})$ species, respectively. ${ }^{41} \mathrm{Bao}$ and $\mathrm{Liu}^{44}$ and Keylor et al. ${ }^{45}$ reported of the chemistry and biology of various derivatives based on Res.

a)

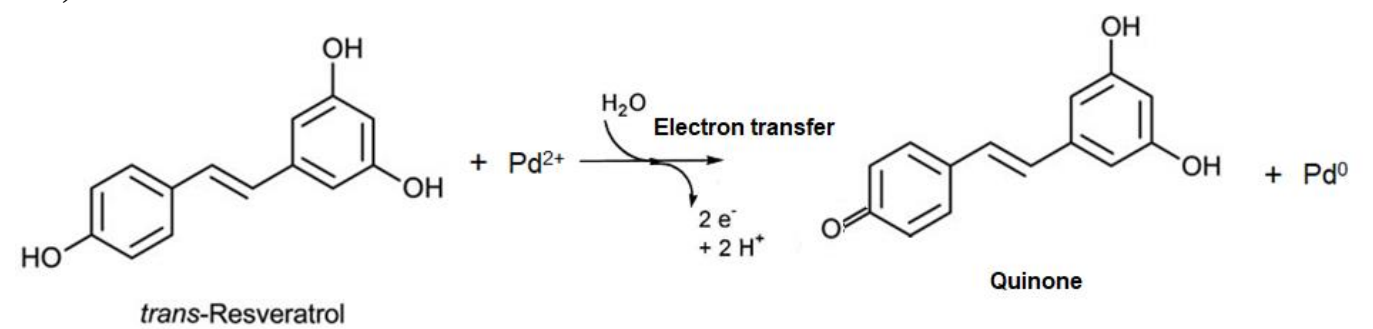

trans-Resveratrol

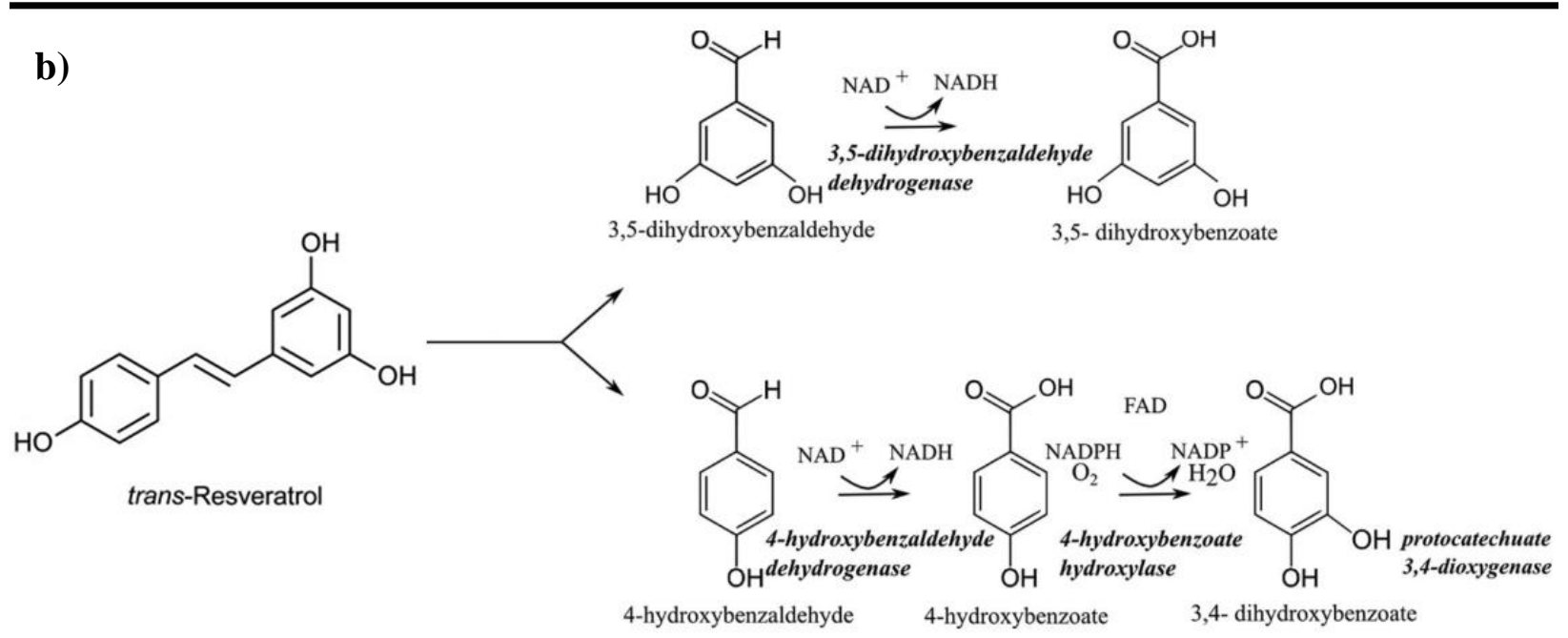

Figure 26: Mechanism for the formation of Res-PdNPs by using Res a) simplified mechanism of Res to quinone form with the reduction of $\mathrm{Pd}^{2+}$ to $\mathrm{Pd}^{\mathbf{0}}$ by electron transfer and b) multiple oxidative products of Res.

Here there is oxidation at only one site, $\mathrm{C}-\mathrm{H}$ changing to $\mathrm{C}-\mathrm{OH}$, change in carbon oxidation state is -1 to +1 . A 2 electron change. Correspondingly the $\mathrm{Pd}^{2+}$ goes to $\mathrm{Pd}^{0}$, also a 2 electron reduction. The ratio of moles of resveratrol: $\mathrm{Pd}^{2+}$ is 1:1. For the Res-PdNPs: Total grams of resveratrol used is $0.33 \mathrm{mg} / \mathrm{mL}$ for $6 \mathrm{~mL}$ which is a total of $0.00198 \mathrm{~g}$ of resveratrol, therefore, 
total moles of resveratrol used is $0.00198 \mathrm{~g} / 228.25 \mathrm{~g} / \mathrm{mol}=9 \times 10^{-6}$ moles and total amount of $\mathrm{Na}_{2} \mathrm{PdCl}_{4}$ used is $100 \mu \mathrm{L}\left(1 \times 10^{-4} \mathrm{~L}\right)$ of $0.1 \mathrm{M}$. Hence moles of $\mathrm{Na}_{2} \mathrm{PdCl}_{4}$ or $\mathrm{Pd}^{2+}$ is $1 \times 10^{-4} \mathrm{~L} \times 0.1$ $\mathrm{M}=1 \times 10^{-5}$ moles. The moles of resveratrol $\left(9 \times 10^{-6}\right.$ moles $)$ and $\mathrm{Pd}^{2+}\left(1 \times 10^{-5}\right.$ moles $)$ is close to $1: 1$, based on this most of Res is oxidized. This represents similar work by Palliyarayil et al. ${ }^{41}$ reporting that the formation of PdNPs follows a 1:1 reaction using (+)-Catechin as a reducing agent.

\subsection{Cellular internalization study}

The cellular internalization of Res-PdNP-4 was investigated against prostate cancer (PC-3) cells at $42 \mu \mathrm{g} / \mathrm{mL}$. The PC-3 cells were incubated with Res-PdNP-4 for 2, 6 and $24 \mathrm{~h}$ to be evaluated using dark field optical microcopy (CytoViva). The CytoViva images revealed that cellular internalization of Res-PdNP-4 is time-dependent with optimal uptake achieved at $24 \mathrm{~h}$ incubation (Figure 27). ${ }^{46}$ The CytoViva images are limited to a two-dimensional field-of-view, with a level of uncertainly whether the Re-PdNP-4 nanoparticles are on the surface of the cells or they are indeed internalized into the cell. The images in Figure 27 show that the Res-PdNP-4 are

contained within the cytoplasm and localized around the nucleus (DAPI stained - blue). ${ }^{47,48}$

Confocal microscopy was used to acquire a three-dimensional field-of-view. A 3D projection snapchat image revealed that the Res-PdNP-4 was localized inside the cytoplasm as shown in Figure 28. The CytoViva and confocal microscopy images of the Res-PdNP-4 seem to be aggregated in the cytoplasm; however, that was due to lower magnification. Thus, TEM was used to further investigate the integrity of the Res-PdNP-4 inside the cell. TEM image analysis of PC-3 cells unambiguously confirmed the internalization and localization of Res-PdNP-4 within PC-3 cancer cells. Moreover, the early endocytic pathway reveals the presence of pseudopodium 
for the internalization of Res-PdNP-4. ${ }^{49}$ These results are in accordance with previous studies in that the Res-PdNP-4 were mostly encapsulated in the autophagosome-like vesicles in the cytoplasm (Figures 29 and 30). ${ }^{48,50,51}$ The trajectory of the Res-PdNP-4's ability to escape the endosomal pathways within the PC-3 cells guarantees a successful delivery of Res-derived phenols and polyphenols encapsulated on the surface of the PdNPs nanocarrier platform. ${ }^{51}$

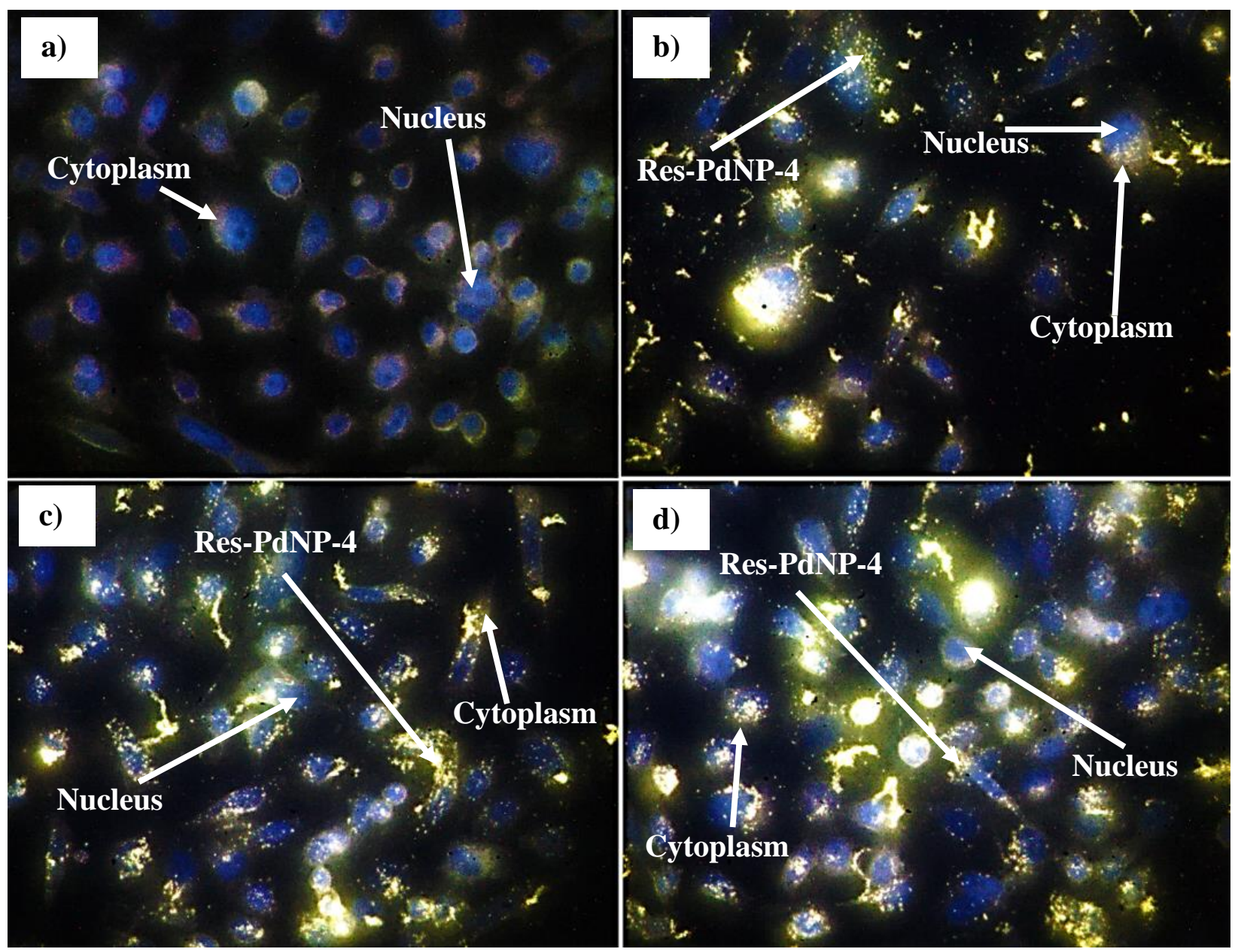

Figure 27: Dark field CytoViva images of prostate cancer (PC-3) cells showing cellular internalization post treatment a) control (no treatment), b) PC-3 cells incubated for 2 h, c) PC-3 cells incubated for $6 \mathrm{~h}$ and d) PC-3 cells incubated with $42 \mu \mathrm{g} / \mathrm{mL}$ Res-PdNP-4 for 24 h. 

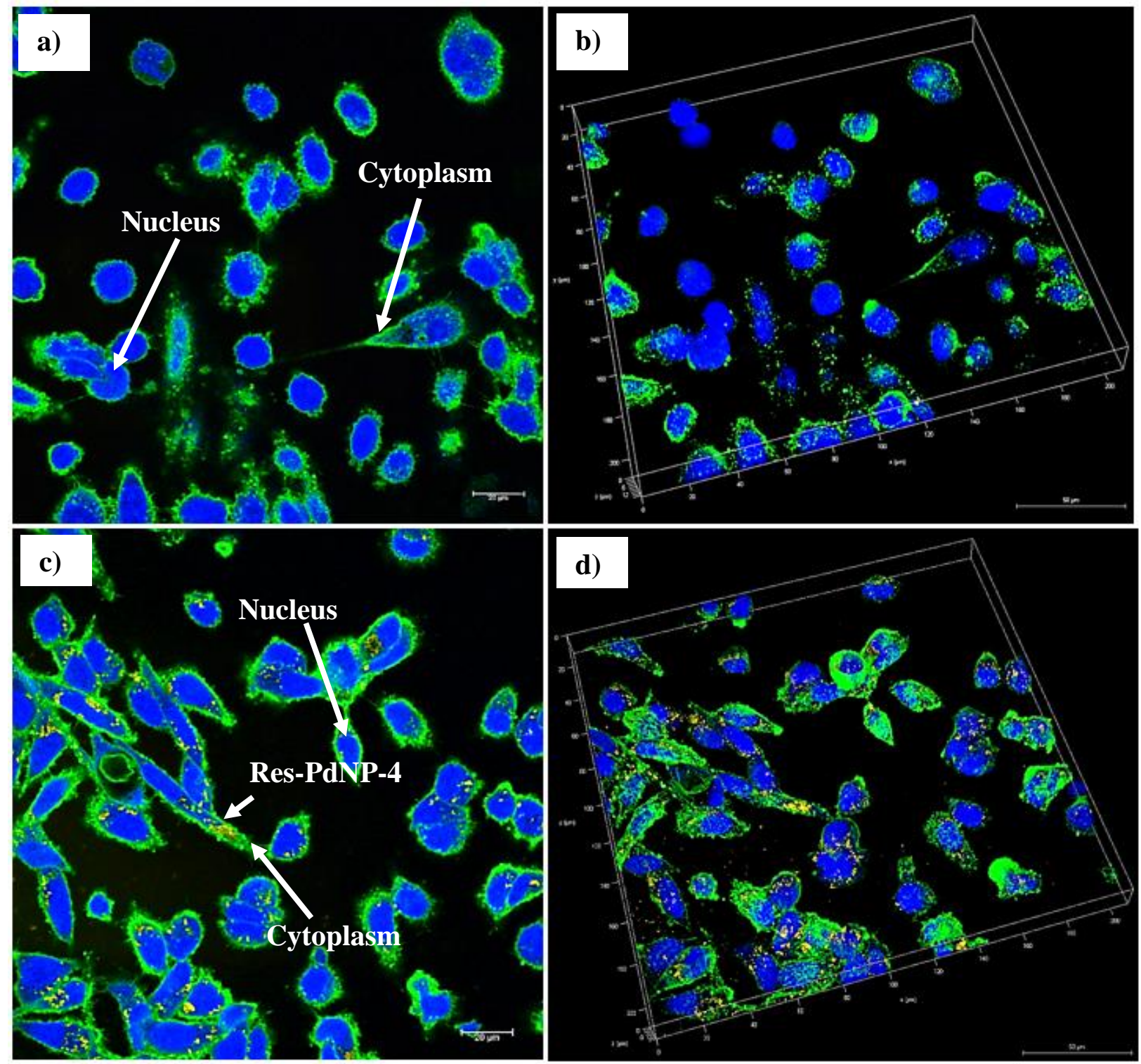

Figure 28: Confocal microscopy images of a) PC-3 control cells (no treatment) and b) 3D projection of a), c) PC-3 cells incubated with $42 \mu \mathrm{g} / \mathrm{mL}$ Res-PdNP-4 for $6 \mathrm{~h}$ and d) 3D projection of c). Nucleus (DAPI stain - blue), cytoplasm (WGA stain - green) and ResPdNP-4 (yellow). 

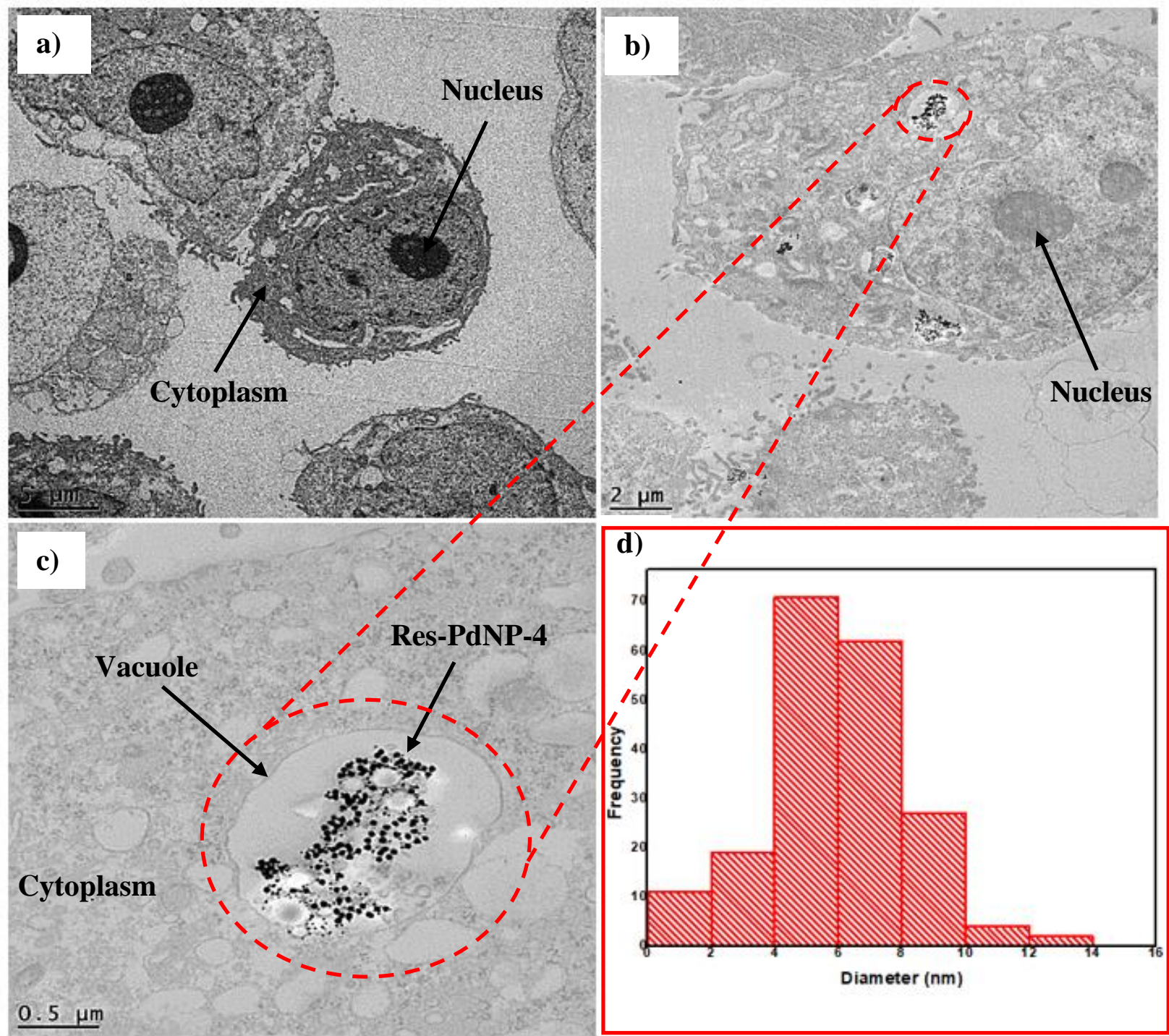

Figure 29: TEM images indicating cellular internalization of Res-PdNP-4 at 6 h post treatment a) PC-3 control cells (no treatment), b) PC-3 cells treated with $42 \mu \mathrm{g} / \mathrm{mL}$ ResPdNP-4, c) magnified image of b) and d) size distribution histogram of Res-PdNP-4. 


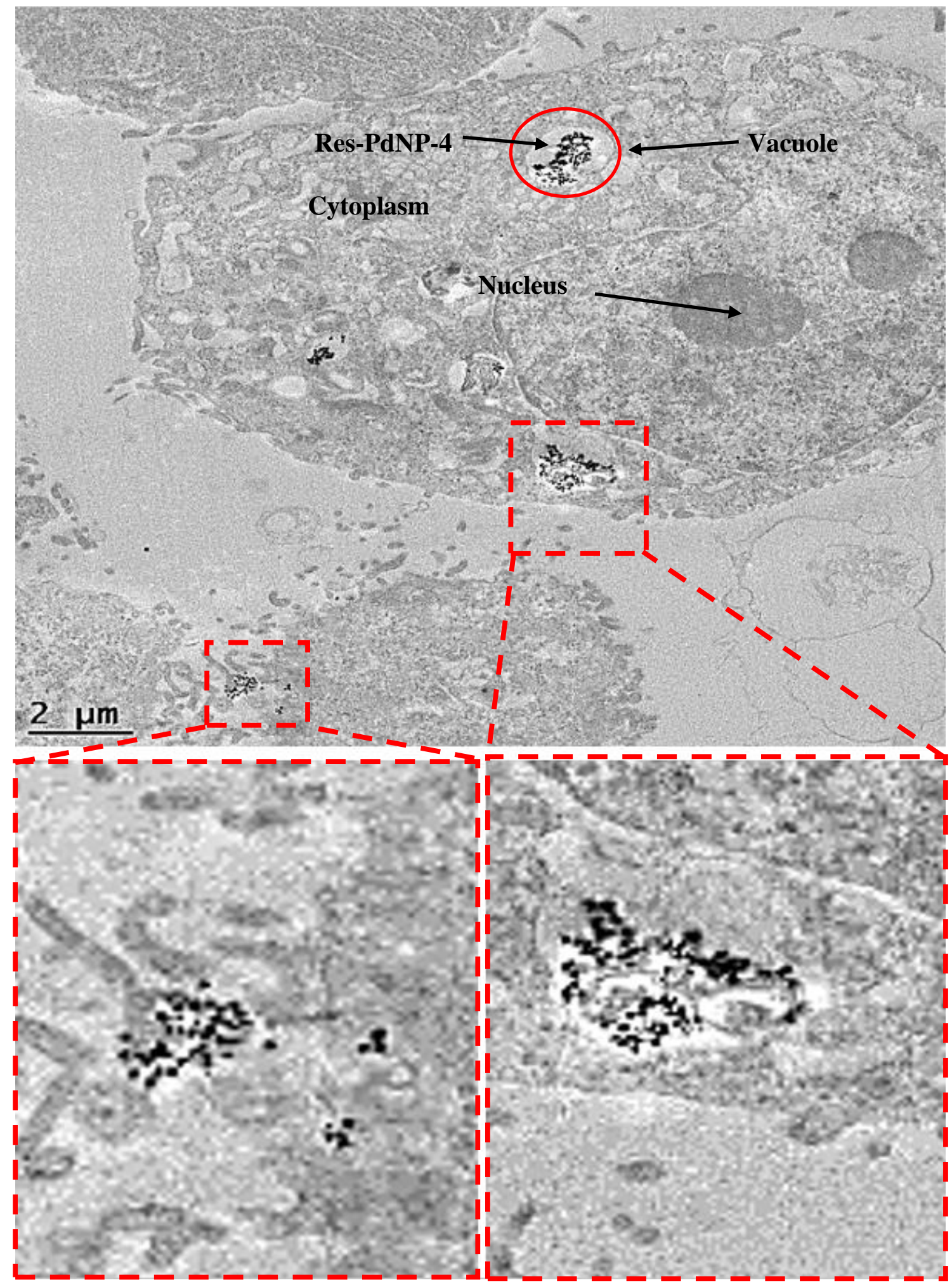

Figure 30: TEM images indicating cellular internalization through endocytic pathway. 42 $\mu \mathrm{g} / \mathrm{mL}$ Res-PdNP-4 at $6 \mathrm{~h}$ post treatment in PC-3 cancer cells. 


\subsection{Receptor mediated cellular internalization of Res-PdNP-4}

The CytoViva, confocal microscopy and TEM images clearly demonstrate that Res-PdNP-4 are internalized into prostate cancer cells with high stability and maintain their integrity inside the cells with no tendency to aggregate inside the cell confirming in vitro and in vivo stability. The mode of internalization was not confirmed at this point, however TEM images confirmed an endocytic pathway based on the formation of an autophagosome-like vacuole containing ResPdNP-4 inside the cytoplasm as shown in Figures 29 and 30. This prompted further investigation whether Res-PdNP-4 cellular internalization is receptor-mediated. The images obtained were comparable with previously reported images in the literature. ${ }^{46-49,51-55}$

Res-PdNP-4 targeting capabilities were investigated towards the laminin receptors (Lam 67R), which are over expressed on human prostate cancer cells and breast and colon cancer. ${ }^{47,56-57}$ Studies by Shukla et al. ${ }^{47}$ and Khoobchandani et al. ${ }^{48}$ revealed that Epigallocatechin gallate (EGCG) conjugated gold nanoparticles (EGCG-AuNPs) demonstrated receptor-mediated internalization through the Lam 67R receptor in prostate cancer cells. Most recently, work by

Diaz-Quiñone et al. $^{56}$ reported that compounds against laminin receptor exhibit anti-cancer properties in prostate cancer cells. Therefore, we hypothesized that Res-PdNP-4 internalization in prostate cancer (PC-3) cells through endocytosis as shown by the TEM images would be mediated through Lam 67R receptor expression.

Blocking studies were performed by confocal microscopy using MLuC5 antibody as a probe. The prostate cancer (PC-3) cells were incubated with MLuC5 to block Lam 67R receptors and the cells were treated with Res-PdNP-4. Confocal images revealed that Res-PdNP-4 were not internalized in the PC-3, which demonstrates that Res-PdNP-4 have Lam 67R receptor affinity 
(Figure 31), thus validating the hypothesis of prostate tumor specific receptor-mediated internalization. Scanning tunnelling electron microscopy (STEM) and energy-dispersive X-ray spectroscopy (EDS) mapping was performed on the Res-PdNP-4 internalized in the prostate cancer (PC-3) cells, confirming that the internalized nanoparticles are indeed Res-PdNP-4 as shown in Figure 32.

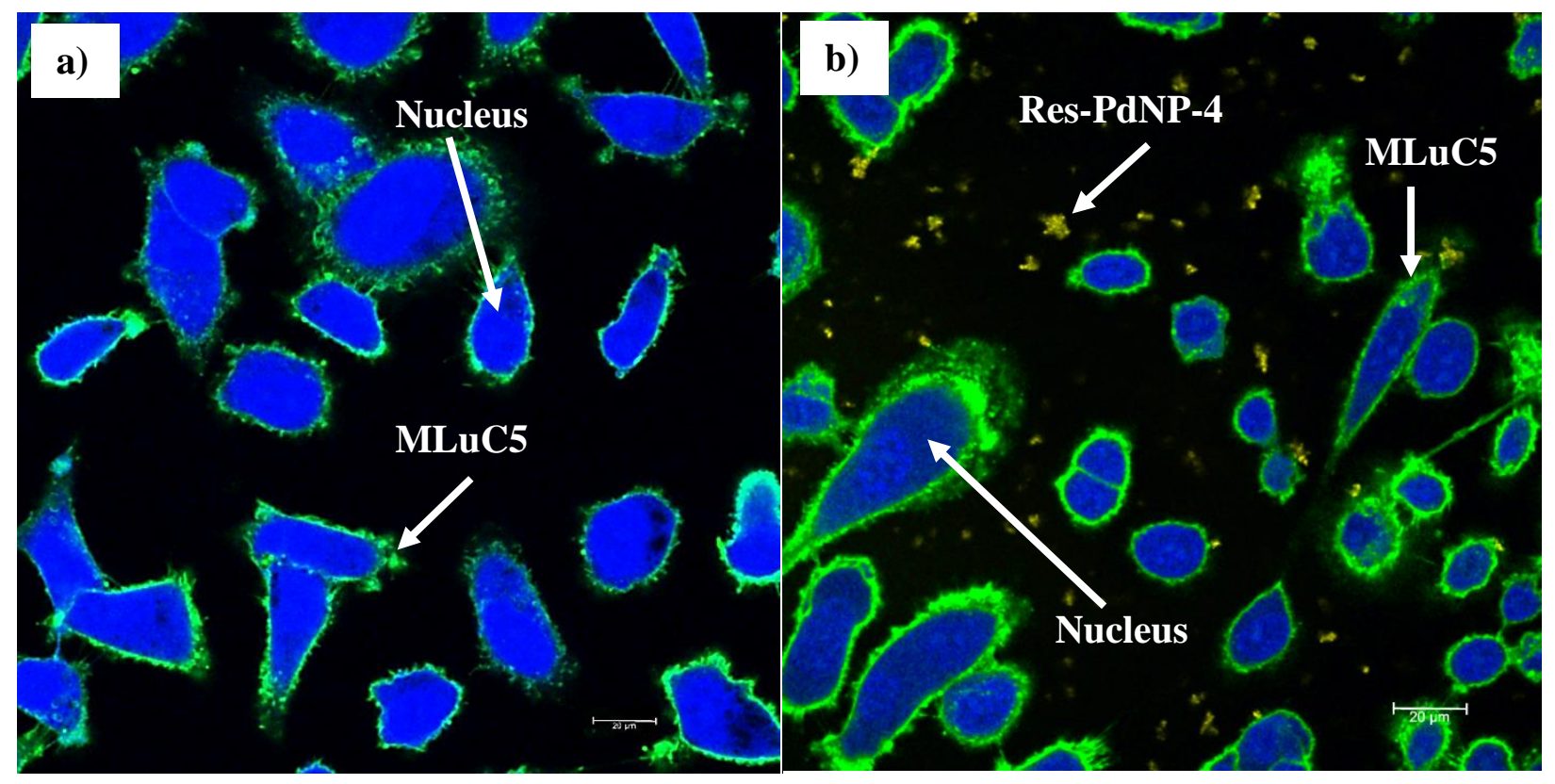

Figure 31: Confocal microscopic images showing immunocytochemical localization of Lam 67R a) prostate cancer (PC-3) control cells (no treatment) and b) inhibition in Res-PdNP-4 uptake in the presence of Laminin 67 receptor blocking antibody (MLuC5) at 6 h post treatment in PC-3 cancer cells. 

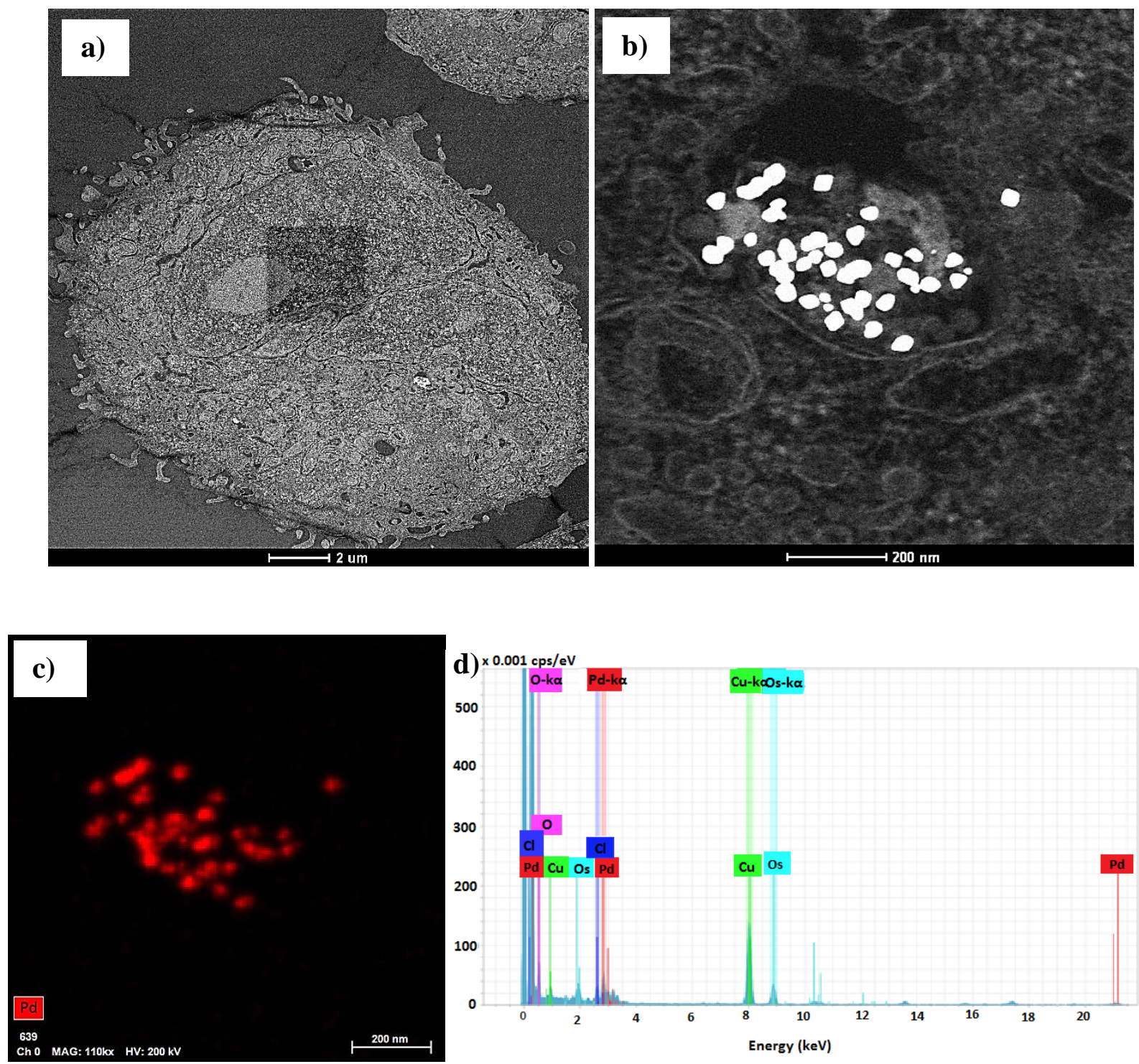

Figure 32: STEM and EDS mapping of prostate cancer (PC-3) cells treated with $42 \mu \mathrm{g} / \mathrm{mL}$ Res-PdNP-4 a) dark-field image of PC-3 cells; b) magnified image of a) showing a vaculoe with nanoparticles; c) EDS mapping of Res-PdNP-4 and d) EDS spectrum confirming the presence of Pd inside the prostate cancer cells. 


\subsection{Assessment of the cytotoxicity of Res-PdNP-4 against prostate cancer (PC-3) cells and normal human aortic endothelial cells (HAEC)}

The cytotoxicity of Res-PdNP-4 was studied on prostate cancer (PC-3) cells as well as on human aortic endothelial cells (HAEC) to investigate the biocompatibility of Res-PdNP-4 via in vitro assay using colorimetric cell viability (MTT) assay. In principle, viable cells are capable of metabolizing the tetrazolium dye MTT 3-(4,5-dimethylthiazol-2-yl)-2,5-diphenyltetrazolium bromide to its insoluble formazan, which has a purple color. The experiment was performed by the treating PC-3 and HAEC cells with Res-PdNP-4 concentrations $(0,2.75,5.5,11,22$ and 44 $\mu \mathrm{g} / \mathrm{mL}$ ). Alongside the treatment with Res-PdNP-4, the cells were also treated with free Res and GA, cisplatin (Pt-based chemotherapeutic drug), etoposide (plant-derived chemotherapeutic drug) and GA-PdNPs as controls. The cellular viability of prostate cancer (PC-3) against ResPdNP-4 after $72 \mathrm{~h}$ of post treatment demonstrated that Res-PdNP-4 exhibit comparable anticancer efficacy as cisplatin and etoposide (Figure 33) with the half maximal inhibitory concentrations $\left(\mathrm{IC}_{50}\right)$ data summarized in Table 7. Please refer to the appendix (Figures 41 and 42) for the results of the cellular viability of PC-3 cells at 24 and $48 \mathrm{~h}$ post treatments.

The free GA and GA-PdNPs had no effect on the PC-3 cells in all time-points, this was because GA is non-toxic as reported in literature. ${ }^{58,59}$ Moreover, recent studies showed that GA has the ability to prevent the toxicity of potent chemotherapeutic drugs such as cisplatin, cyclophosphamide and doxorubicin. ${ }^{60}$ Kannan et al. ${ }^{61}$ and Axiak-Bechtel et al. ${ }^{62}$ reported that GA provides a nontoxic coating that prevents nanoparticle aggregation in vivo. A number of $\mathrm{Pd}$ complexes and compounds $[\mathrm{Pd}(\mathrm{I})$ and $\mathrm{Pd}(\mathrm{II})]$ have been reported to be toxic. ${ }^{63-65}$ However, in the current study, GA-PdNPs were not toxic, this could be attributed to inert Pd (0 oxidation 
state). This suggests that the increased Res corona on the Res-PdNP-4 has a synergistic effect facilitated by PdNPs being effective carriers to enhance the bioavailability of Res, validating the therapeutic potential of Res-PdNP-4 for treating prostate cancer through adjuvant therapeutic benefits of inherent anti-angiogenesis and pro-apoptotic properties of PdNPs and anti-tumor characteristics of Res - providing an additional armamentarium in tumor therapy.

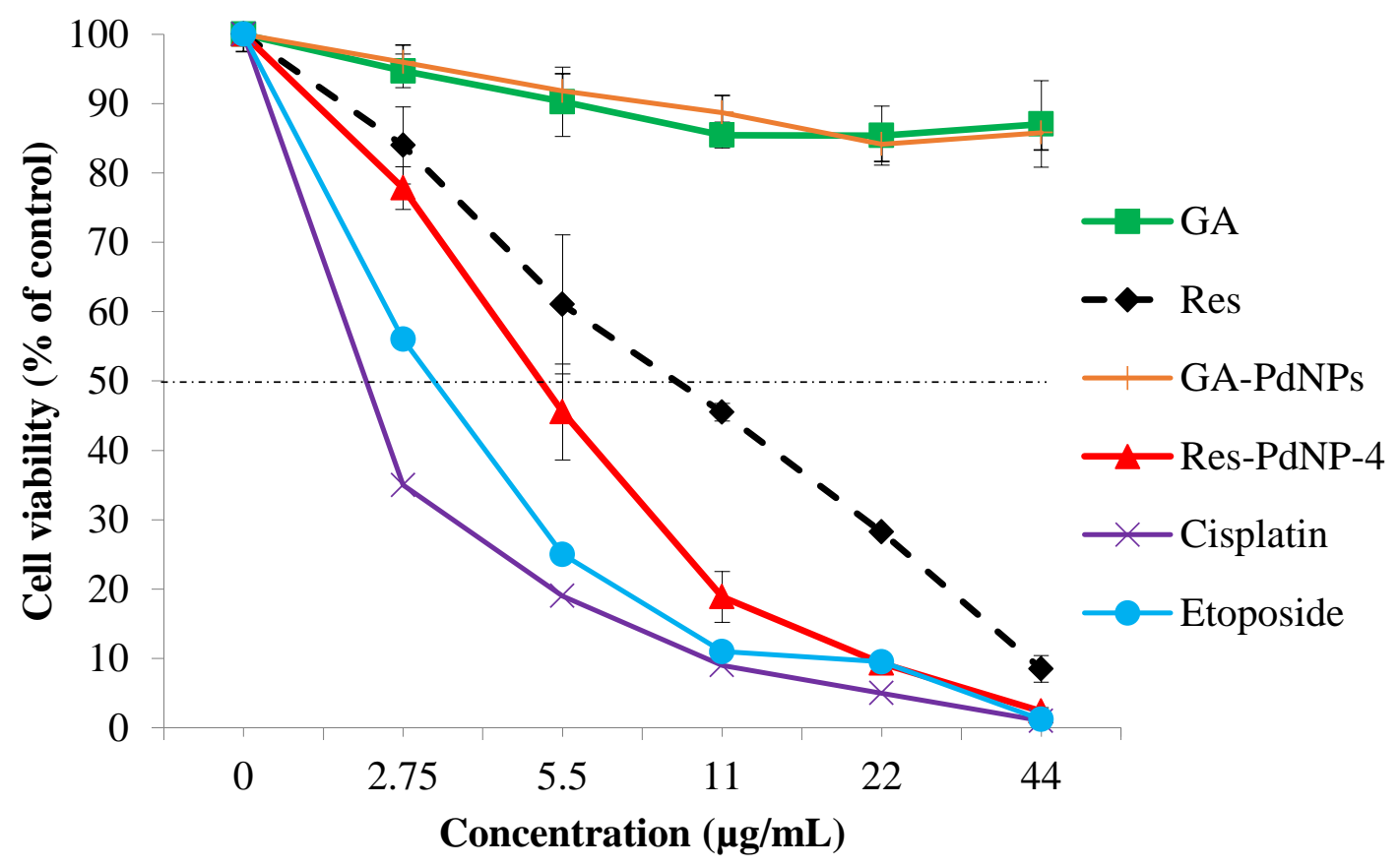

Figure 33: Cell viability of prostate cancer (PC-3) after $72 \mathrm{~h}$ post incubation with ResPdNP-4 and controls (free Res, GA, GA-PdNPs, cisplatin and etoposide), n=4; results reported as mean \pm SEM.

Based on the exceptional cytotoxic data of Res-PdNP-4 against prostate cancer (PC-3) cells, the Res-PdNP-4 nanoparticles were further investigated against normal human aortic endothelial cells (HAEC). From the cellular internalization studies by confocal microscopy and TEM revealed that Res-PdNP-4 has Lam 67R receptor affinity, which are receptors overexpressed in prostate cancer than normal cells. The HAEC cells were incubated with Res-PdNP-4, cisplatin 
and etoposide at different concentrations $(0,2.75,5.5,11,22$ and $44 \mu \mathrm{g} / \mathrm{mL})$ for $72 \mathrm{~h}$ post treatments. As expected, cisplatin and etoposide treatments were highly toxic to HAEC due to the toxicity profile while Res-PdNP-4 presented no toxicity further corroborating Lam 67R receptor-mediated delivery, making them specific to cancerous cells as shown in Figure 34. Please refer to the appendix (Figure 42) for the results of the cellular viability HAEC cells at 24 and $48 \mathrm{~h}$ post treatments.

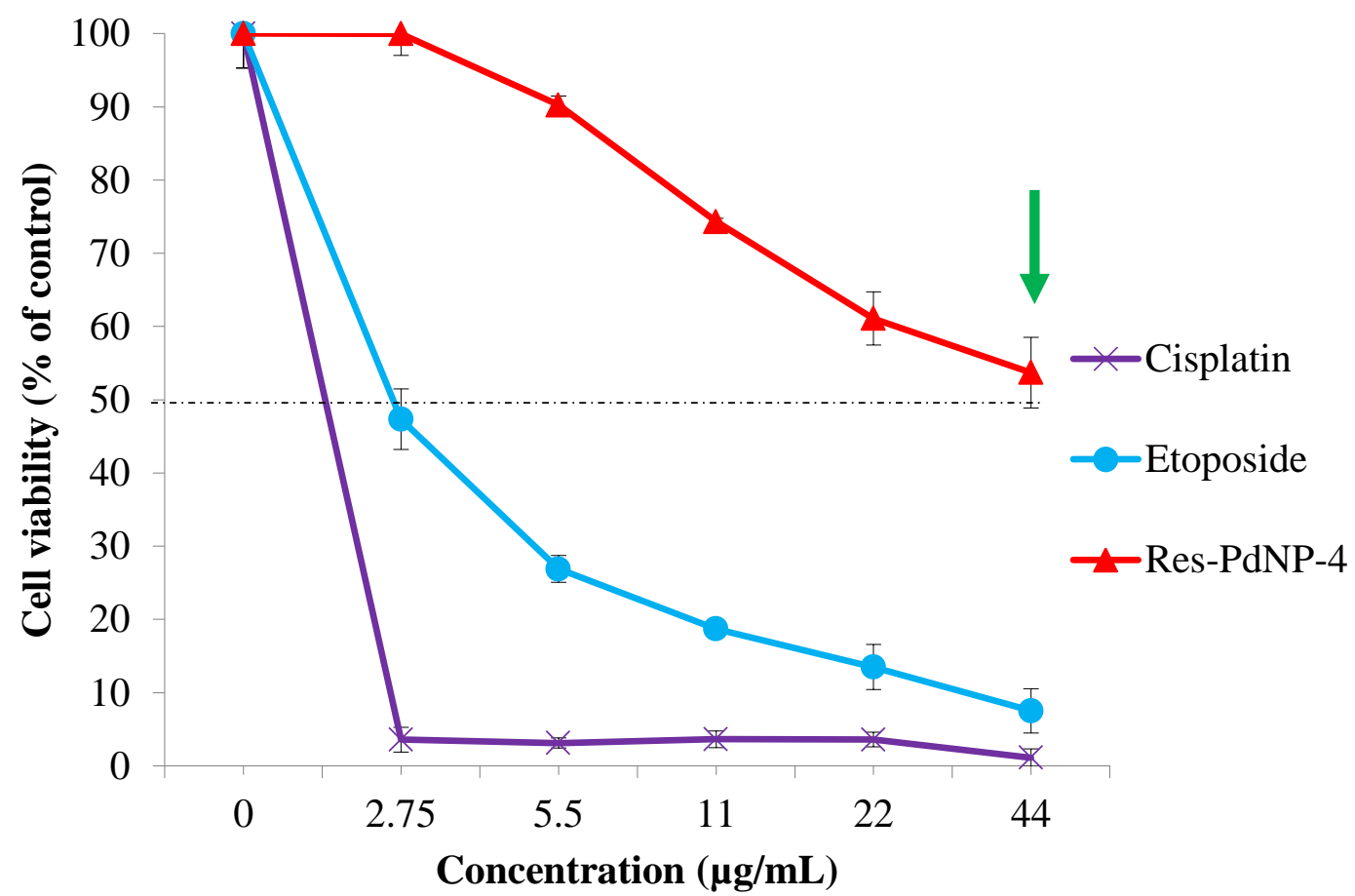

Figure 34: Cell viability of normal human aortic endothelial cells (HAEC) after 72 h post incubation with Res-PdNP-4 and controls (cisplatin and etoposide). At high Res-PdNP-4 concentration no toxicity was observed (green arrow) $n=4$; results reported as mean \pm SEM. 
Table 7: in vitro cellular viability studies

\begin{tabular}{|c|c|c|c|c|}
\hline \multirow{2}{*}{ Sample } & \multirow{2}{*}{ Cell line } & \multicolumn{3}{|c|}{$\mathrm{IC}_{50}(\mu \mathrm{g} / \mathrm{mL})$} \\
\hline & & $24 \mathrm{~h}$ & $48 h$ & $72 \mathrm{~h}$ \\
\hline Cisplatin & \multirow{3}{*}{ Prostate cancer (PC-3) } & 2 & 2 & 2 \\
\hline Etoposide & & N/A & 5 & 3 \\
\hline Res-PdNP-4 & & 10 & 7 & 5 \\
\hline Cisplatin & \multirow{3}{*}{$\begin{array}{l}\text { Normal human aortic } \\
\text { endothelial cells (HAEC) }\end{array}$} & 3 & 2 & 2 \\
\hline Etoposide & & 2 & 3 & 3 \\
\hline Res-PdNP-4 & & 15 & N/A & N/A \\
\hline
\end{tabular}

${ }_{50}{ }_{50}$ half maximal inhibitory concentration, ${ }^{\mathrm{N} / \mathrm{A}}$ no $50 \%$ inhibition

\subsection{Cellular morphology and migration studies}

The cellular morphology of prostate cancer (PC-3) cells and normal human aortic endothelial cells (HAEC) was investigated. The PC-3 cells treated with Res-PdNP-4, cisplatin and etoposide incubated for $72 \mathrm{~h}$ post treatment at $42 \mu \mathrm{g} / \mathrm{mL}$ showed dramatic morphological changes, which is an initial indication of the PC-3 cells undergoing apoptosis. This was evident due to the presence cell shrinkage and cell debris confirming morphological characteristics of apoptosis (Figure 35). The treatment of HAEC cells with Res-PdNP-4 was identical to the control (no treatment) which revealed that Res-PdNP-4 present no toxic effect towards normal cells; however, that is not true 
for cisplatin and etoposide since they are highly toxic towards normal cells (Figure 36). This data is in total agreement with the in vitro cellular viability results. This data is very convincing that Res-PdNP-4 is comparable to data as observed with cisplatin and etoposide in PC-3 cells. However, Res-PdNP-4's low toxicity towards normal cells makes it an ideal cancer therapy.
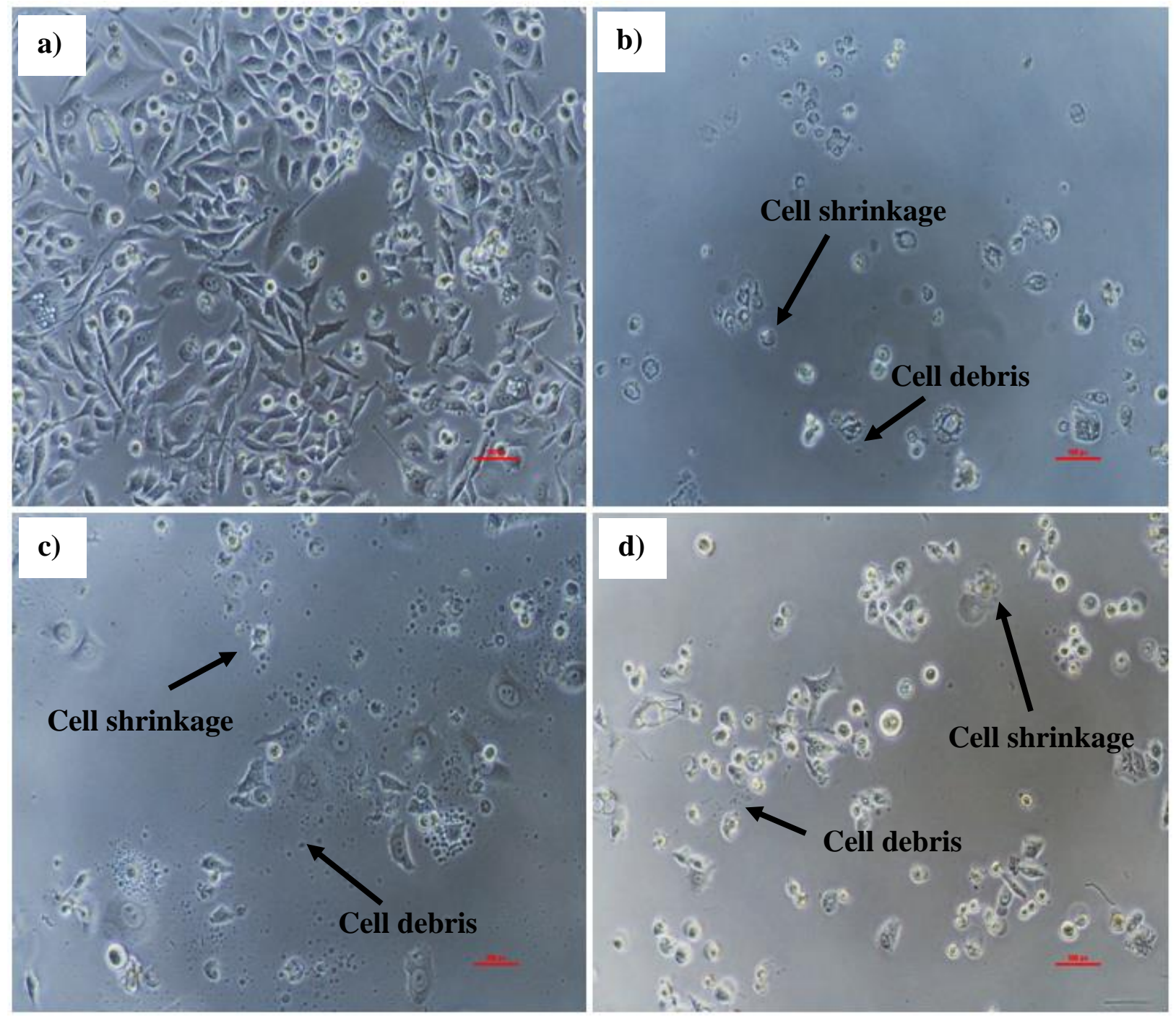

Figure 35: Cellular morphological studies of prostate cancer (PC-3) cells after 72 h post incubation with a) control (no treatment), b) cisplatin, c) etoposide and d) Res-PdNP-4 for $72 \mathrm{~h}$ at $42 \mu \mathrm{g} / \mathrm{mL}$. 

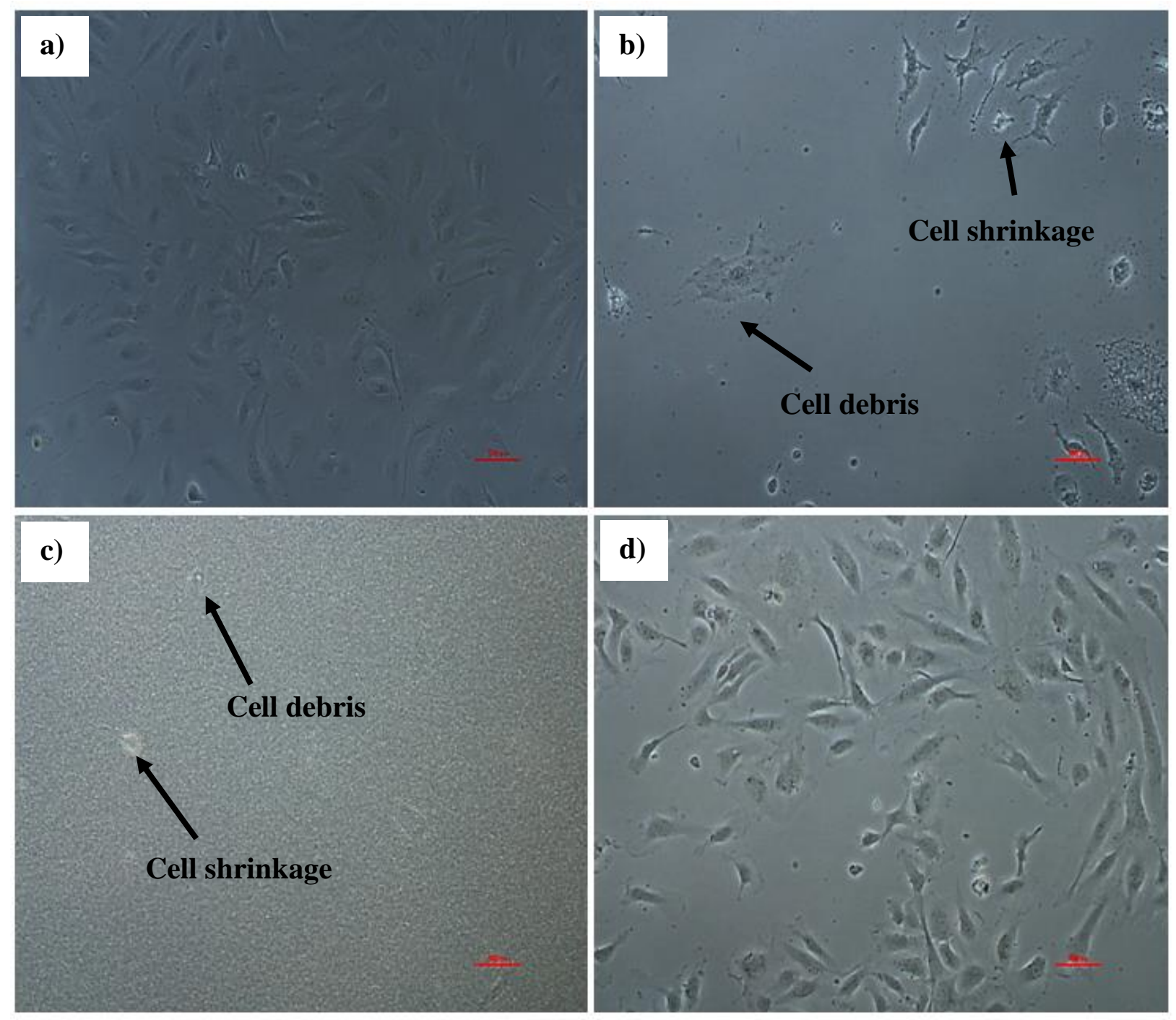

Figure 36: Cellular morphological studies of normal human aortic endothelial cells (HAEC) after $72 \mathrm{~h}$ post incubation with a) control (no treatment), b) cisplatin, c) etoposide and d) Res-PdNP-4 for $72 \mathrm{~h}$ at $42 \mu \mathrm{g} / \mathrm{mL}$.

Cellular migration studies of prostate cancer (PC-3) treated with Res-PdNP-4 revealed that the Res-PdNP-4 have the ability to stop cellular migration and provides preliminary evidence of Res-PdNP-4 to limit tumor metastasis (Figure 37). 

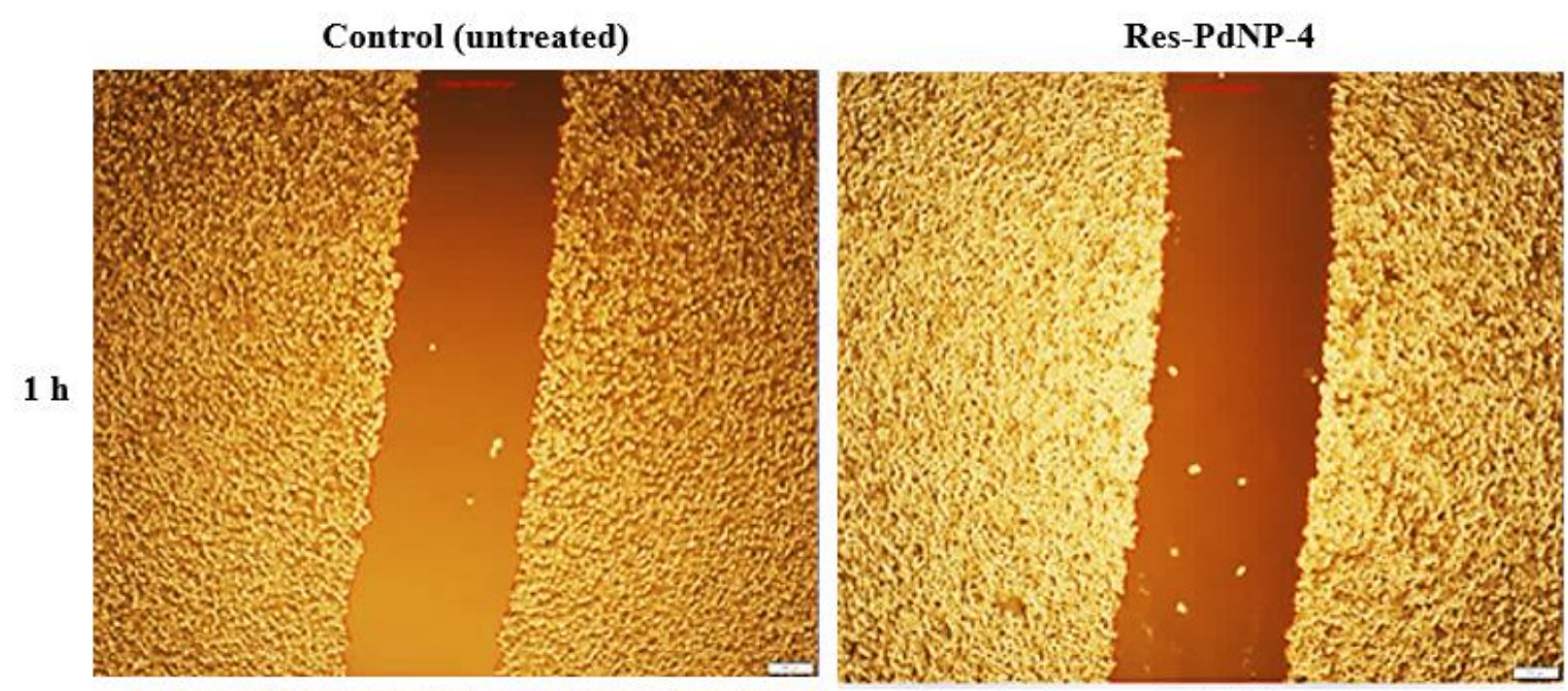

24 h
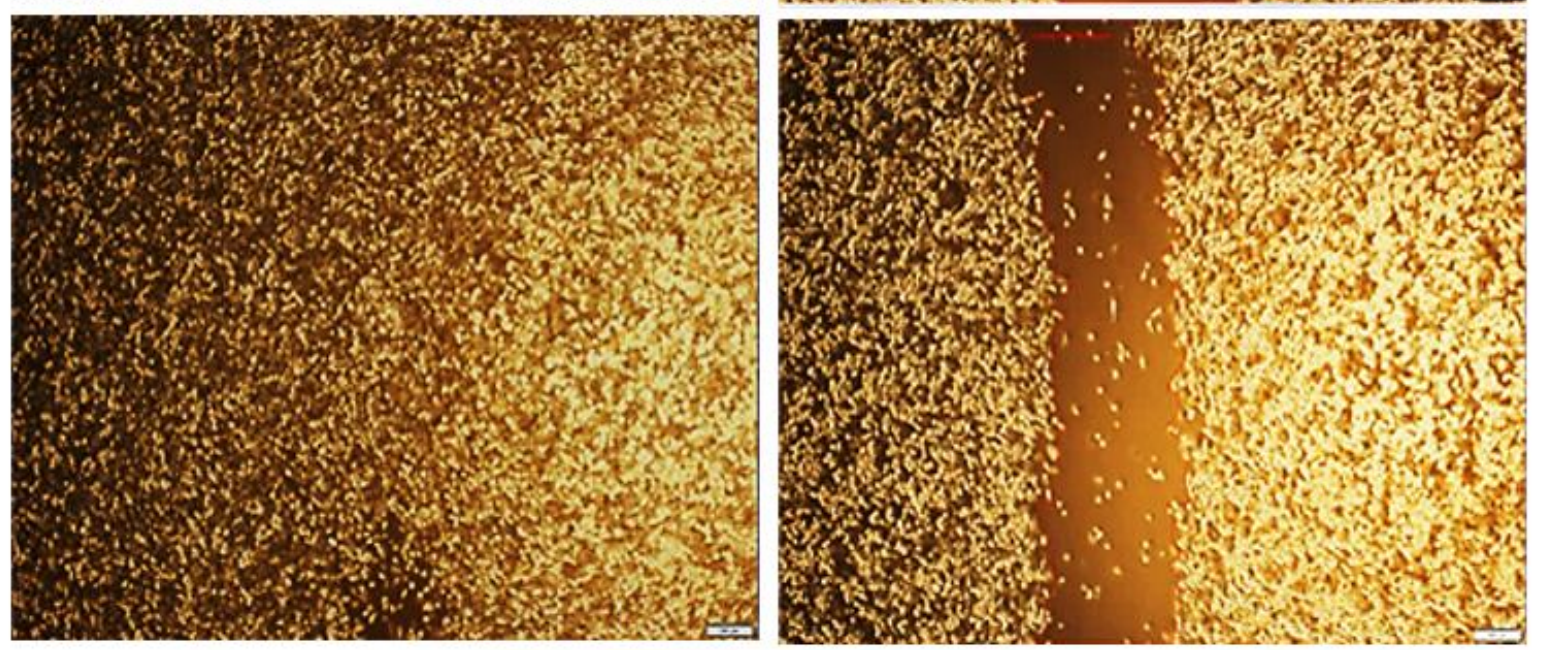

Figure 37: Cellular migration study of prostate cancer (PC-3) cells treated with Res-PdNP-

4 compared with control cells (no treatment). 


\subsection{Therapeutic efficacy study of Res-PdNP-4}

The therapeutic efficacy study of Res-PdNP-4 (increased Res corona with GA) was investigated using human prostate tumor-bearing severely combined immunodeficient (SCID) male mice as the animal model to evaluate Res-PdNP-4's ability to control or reduce tumor size. There were three groups of mice $(\mathrm{n}=5)$ bearing prostate $(\mathrm{PC}-3)$ tumors with comparable size (the mean tumor volume ranging from $0.02-0.1 \mathrm{~cm}^{3}$ ) and mean body weights (bw) ranged from 28-31.2 $\mathrm{g}$. After inoculation, the animals were treated when the tumors were palpable by intravenous (IV) administration twice a week, at which time the tumors were measured by digital caliper measurements and calculated as length $\mathrm{x}$ width $\mathrm{x}$ height. The first group was IV injected with $100 \mu \mathrm{L}$ saline, this served as the control group. The second group was IV injected with $100 \mu \mathrm{L}$ Res-PdNP-4 $(0.25 \mathrm{mg} / \mathrm{kg} \mathrm{bw})$ whereas the third group was IV injected with $100 \mu \mathrm{L}$ free Res $(7$ $\mathrm{mg} / \mathrm{kg} \mathrm{bw})$. An additional control group of normal untreated mice was added to the study, which served as controls for the complete blood count (CBC) values. The study was conducted for 31 days; day 0 was considered the day of randomization and the day of initial treatment.

Results of the therapeutic study indicated that Res-PdNP-4 has the ability to control the tumor volume in comparison to the saline control group and free Res treated group (Figure 38). Approximately after one week of treatment (day 10), the tumor size was controlled by ResPdNP-4 at $0.04 \pm 0.02 \mathrm{~cm}^{3}$ while the group treated with saline $\left(0.05 \pm 0.02 \mathrm{~cm}^{3}\right)$ and free Res $\left(0.08 \pm 0.06 \mathrm{~cm}^{3}\right)$ started to show a slight increase in the tumor size. The tumor size of the first and third group (saline and free Res) at two week post injection (day 17) was much greater than the previous week greater at $0.06 \pm 0.02 \mathrm{~cm}^{3}$ and $0.1 \pm 0.05 \mathrm{~cm}^{3}$, respectively, as compared to the 
Res-PdNP-4 treatment in which the tumor size was controlled at $0.04 \pm 0.02 \mathrm{~cm}^{3}$ as shown in Figure 38.

Four weeks post treatment (day 31), there was a transient increase in the tumor size at $0.2 \pm 0.1$ $\mathrm{cm}^{3}$ and $0.3 \pm 0.05 \mathrm{~cm}^{3}$ for the groups treated with saline and free Res, respectively. The tumor size for Res-PdNP-4 treated group was $0.1 \pm 0.04 \mathrm{~cm}^{3}$, which clearly showed the ability of ResPdNP-4 to control the tumor growth. By day 35, only few animals from all the treated groups remained alive while many animals were euthanized due to tumor burden during the study. From the saline control and free Res groups only 1 out of 5 animals and 3 out of 5 animals from ResPdNP-4 remained alive. The body weights were taken twice a week during treatment until the end of the study, no significant body weight loss was observed for the Res-PdNP-4 treatment compared to the normal healthy control group as shown in Figure 39. The in vivo therapeutic efficacy shows that Res-PdNP-4 are highly effective compared to free Res, which was due to the high corona of Res on the PdNPs and PdNPs facilitated effectively enhanced delivery of Res with high bioavailability, giving an advantage in tumor therapy. A study by Chen et al. ${ }^{66}$ reported that Pd nanosheets in vivo accumulate in the liver and spleen from IV administration which was $\mathrm{Pd}$ nanosheet size and time point post-injection dependent. At 4 days post-injection, the Pd nanosheets were cleared by renal excretion while at 30 days post-injection their data revealed that $13 \mathrm{~nm}$ Pd nanosheets were in the spleen. We believe that the Res-PdNP-4 have a similar route of clearance because of the comparable size with the Pd nanosheets. 


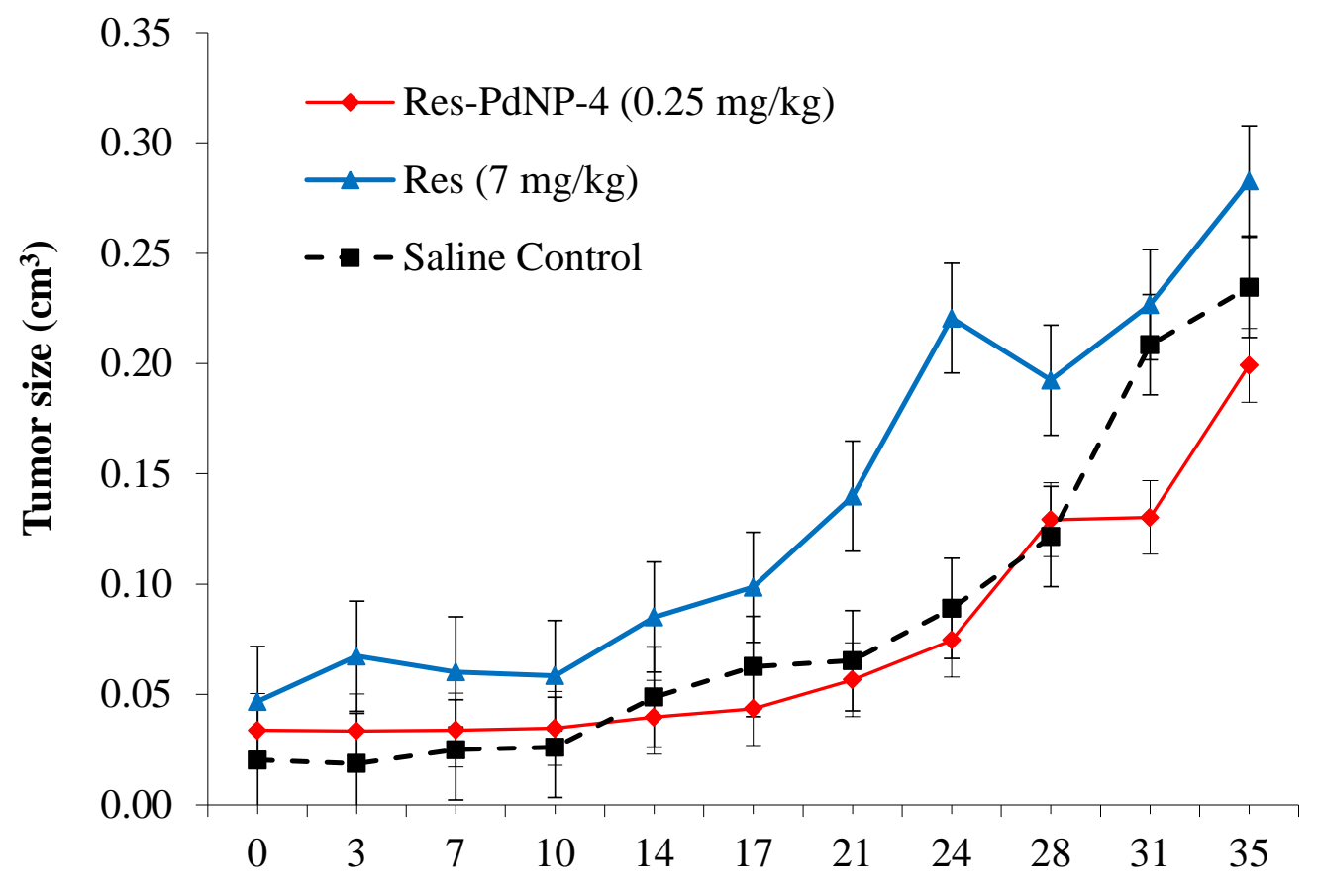

Days after palpable tumors randomized

Figure 38: Therapeutic efficacy studies of prostate tumor-bearing SCID male mice treated by intravenous injection twice a week with Res-PdNP-4, Res and saline. 


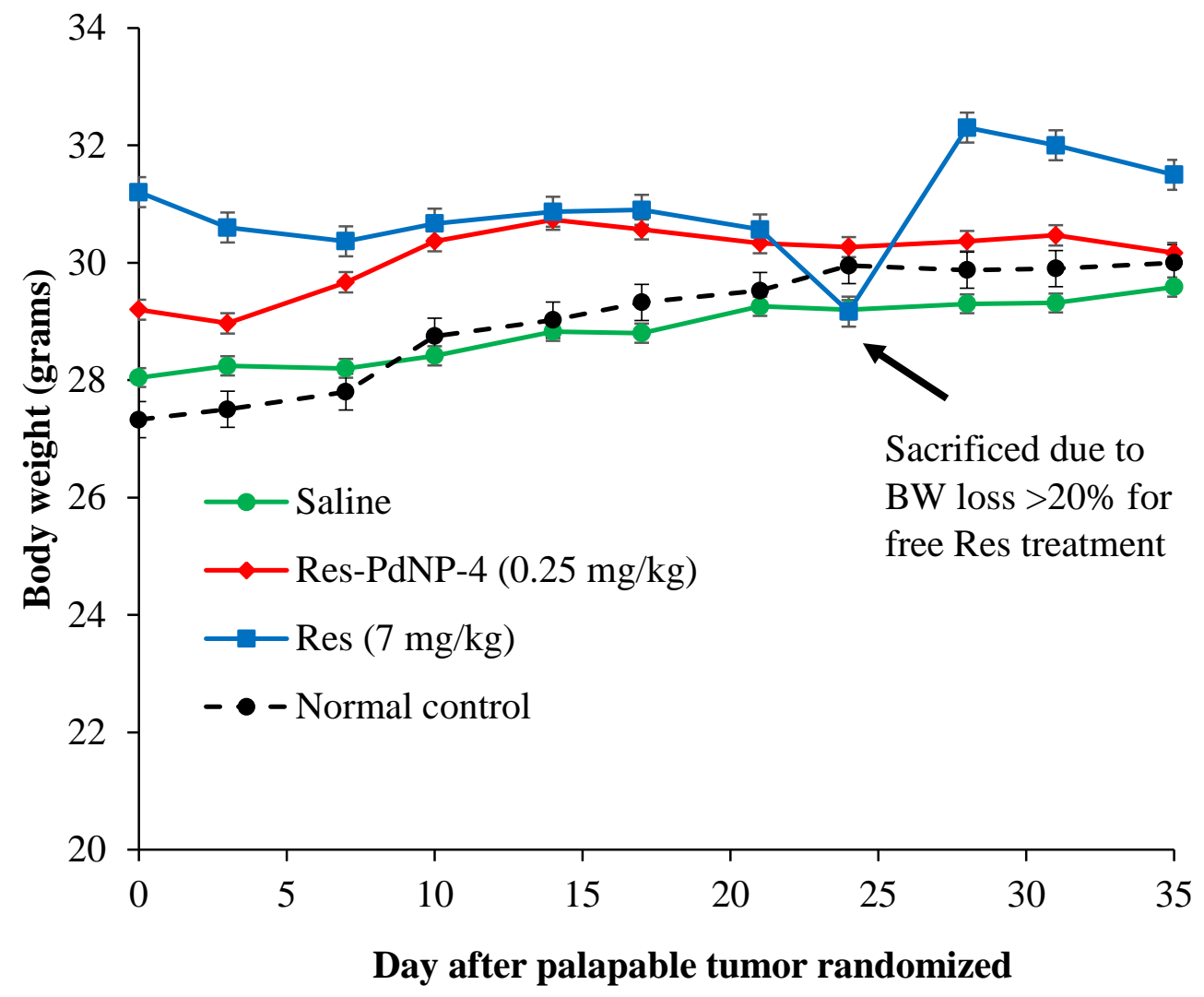

Figure 39: Average body weight of the SCID male mice during the study.

Blood parameters within the tumor-bearing saline, free Res and Res-PdNP-4 treatment groups were compared with baseline levels from a normal healthy group that had not been experimentally manipulated and served as the control for this analysis. The parameters that were compared included mean counts for white blood cells (WBC), red blood cells (RBC) as well as hemoglobin, lymphocytes, and platelets counts. The analysis revealed that the mean WBC count for the Res-PdNP-4 treated group was $2.2 \pm 1.1 \times 10^{3} \mathrm{WBC} / \mu \mathrm{L}$, saline treated group was $3.7 \pm 2.0$ $\mathrm{x} 10^{3} \mathrm{WBC} / \mu \mathrm{L}$ and $3.5 \pm 1.4 \times 10^{3} \mathrm{WBC} / \mu \mathrm{L}$ for free Res treated group. By contrast, the WBC count for the normal control group was $1.6 \pm 0.5 \times 10^{3} \mathrm{WBC} / \mu \mathrm{L}$. There was no significant difference in WBC count among all the treated groups. The mean RBC count, hemoglobin 
(g/dL), lymphocytes and platelets count, revealed that there was no significant differences among treated groups and slightly lower than those of the control group. Moreover, the platelets counts for the control group was $657.5 \pm 212.3 \times 10^{3} / \mu \mathrm{L}$, while the Res-PdNP-4, saline and free Res treated groups was $697.5 \pm 174.6 \times 10^{3} / \mu \mathrm{L}, 900.2 \pm 307.1 \times 10^{3} / \mu \mathrm{L}$ and $847 \pm 217.1 \times 10^{3} / \mu \mathrm{L}$, respectively.

\subsubsection{Immunohistochemistry of tumor xenografts}

Tumor growth is directly proportional to sufficient blood vessels present in the tissue responsible for the blood supply through sprouting angiogenesis and co-option of existing blood vessels. ${ }^{67}$ The mean microvessel density (MVD) was measured to evaluate neovascularization within the tumor xenografts by CD31 staining; this was quantified by ImageJ Immunohistochemistry (IHC) Image Analysis software. The CD31 positively stained vessels in prostate cancer tissue sections are shown in Figure 40a-c, respectively. Res-PdNP-4 treatment revealed a substantial decrease in number of microvessel density (MVD) at $35 \pm 5$ vessels per $\mathrm{mm}^{2}$ compared to the saline and free Res treated groups at $72 \pm 3$ and $85 \pm 5$ vessels per $\mathrm{mm}^{2}$, respectively.

The obtained MVD data are clearly indicative and support the therapeutic efficacy in vivo results that Res-PdNP-4 has the ability to control the tumor volume compared to the saline and free Res treated group. Analysis of tissue CD31 biomarker showed that the Res-PdNP-4 inhibited angiogenesis in tumor tissue. Thus, the results indicated that Res-PdNP-4 was obviously efficient for prostate cancer cells, a synergism of antitumor property of Res-derived phenols and polyphenols with PdNPs nanocarrier delivery for increased Res-derived phenols and polyphenols bioavailability in comparison to the saline control group and free Res treated groups. 

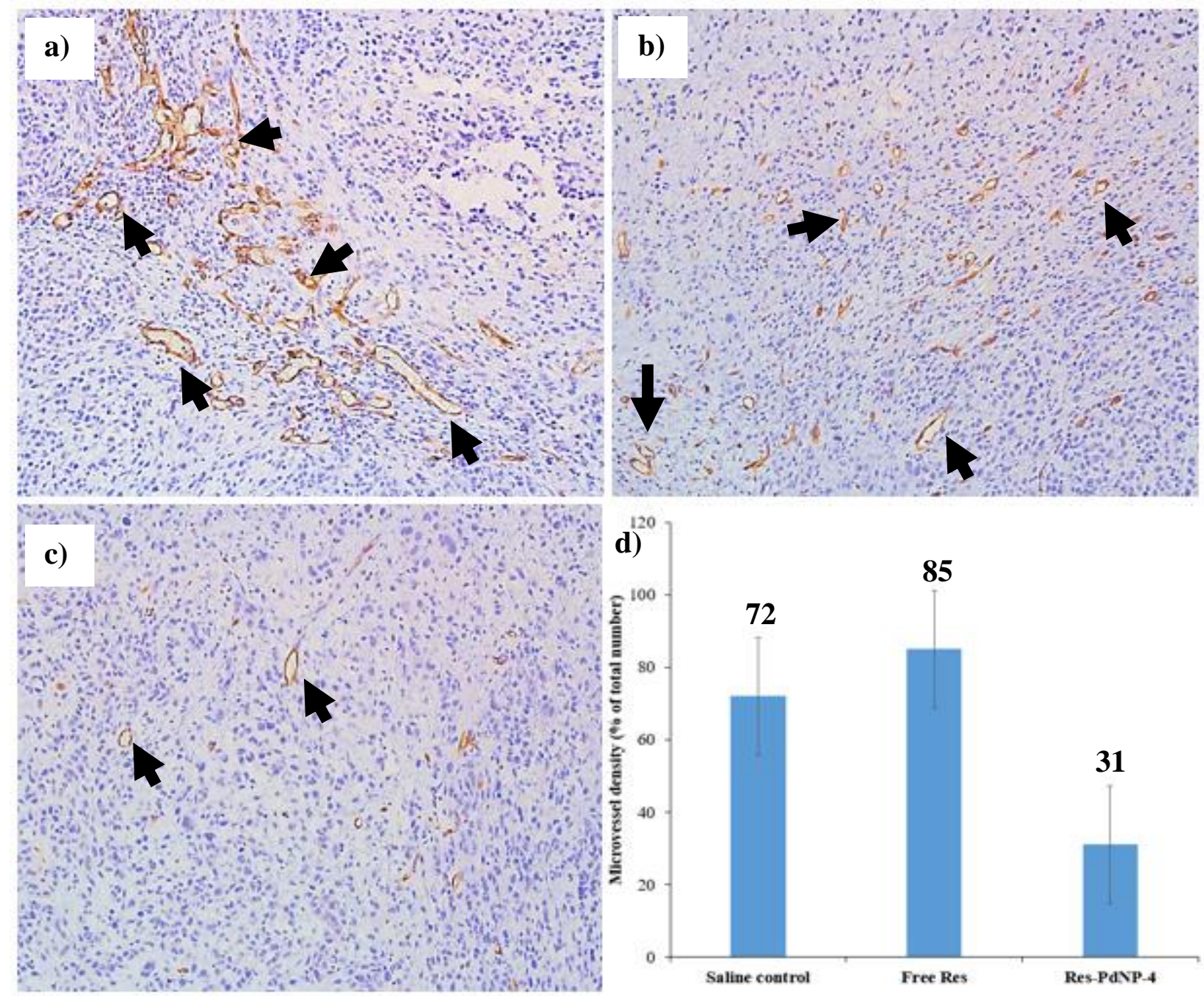

Figure 40: Immunohistochemical CD31 staining of tumor tissues to evaluate blood vessels. Twelve fields from each xenograft were analyzed to determine the average number of vessels per field (microvessel density $=$ MVD). $\mathbf{N}=3$; mean \pm STEM; a) saline control group, b) free Res treated group and c) Res-PdNP-4 treated group. 


\section{CHAPTER 5}

\section{CONCLUSION}

The present research resulted in the development of four types of resveratrol encapsulated palladium nanoparticles: Res-PdNP-1, Res-PdNP-2 (increased Res-derived phenols and polyphenols corona), Res-PdNP-4 (stabilized with GA) and Res-PdNP-4 (increased Res-derived phenols and polyphenols corona stabilized with GA). The production of nanoparticles with a higher Res corona (Res-PdNP-2 and Res-PdNP-4) was not accidental after completing extensive optimization and research on achieving this synthetic route was challenging. The Res-PdNPs can be used for the treatment of prostate cancer through selective and specific target-mediated delivery to achieve a high therapeutic benefit without affecting surrounding healthy tissues.

In the first project of this study, resveratrol served a dual role as a reducing and stabilizing agent for the reduction of $\mathrm{Pd}$ within the $\mathrm{Na}_{2} \mathrm{PdCl}_{4}$ salt to the corresponding palladium nanoparticles. The synthesis route to achieve the synthesis of Res-PdNP-1 was successful. Based on the results obtained of Res-PdNP-1, the amount of Res-derived phenols and polyphenols loaded onto the palladium nanoparticles was less to achieve high therapeutic effect. This prompted further investigation, into increasing the Res-derived phenols and polyphenols corona on the PdNPs. The protocols that were established to synthesize increased Res-derived phenols and polyphenols corona by using more Res in the reaction were successful; this was evident through the production of Res-PdNP-2. The in vitro stability data of Res-PdNP-1 and Res-PdNP-2 revealed that Res-PdNPs were not stable in serum.

In the second project of this study, the development of stable Res-PdNPs using gum arabic (GA). The introduction of GA provided much needed stability to the Res-PdNPs and the Res-PdNP-3 
and Res-PdNP-4 were stable in all biological media tested. Moreover, TEM results revealed that GA was not limited to the stability of the Res-PdNP-3 but also facilitated the crystallization of the produced palladium nanoparticles (Res-PdNP-3 and Res-PdNP-4) and subsequently provided a supportive matrix with higher resveratrol-derived phenols and polyphenols loading capacity. The ${ }^{1} \mathrm{H}$ NMR results demonstrated that oxidized Res, as well as oxidized products (piceatannol, 3,5-dihydroxybenzoic acid, 3,5-dihydroxybenzaldehyde and para-hydroxybenzaldehyde) of Res on the PdNPs. The Res-PdNP-4 was considered the ideal Res-PdNPs for further investigation because of their high stability and Res corona in order to improve the bioavailability, biodistribution and provide an adjuvant therapy to induce selective and specific prostate tumorcell-death. The in vitro cellular results revealed that Res-PdNP-4 were selective to the laminin receptors overexpressed in prostate cancer cells. This was established through cellular internalization results which confirmed Res-PdNP-4 cellular internalization is mediated through an endocytic pathway. The cellular viability results of Res-PdNP-4 nanoparticles exhibited comparable anticancer efficacy against prostate cancer (PC-3) cells as chemotherapy drugs (cisplatin and etoposide), while cisplatin and etoposide treatments were highly toxic to normal HAEC cells due to the toxicity profile. The Res-PdNP-4 is an excellent candidate to be used for treatment of prostate cancer because they presented no toxicity against HAEC cells.

In the third project of this study, the therapeutic efficacy results showed that Res-PdNP-4 nanoparticles have significant therapeutic effect and are able to control the tumor size in comparison to the control and free resveratrol treated groups. It is remarkable to conclude from these results that tumor selective affinity of Res-PdNP-4 provides an additional armamentarium for therapy through antitumor property of high Res corona and adjuvant therapeutic benefits through PdNPs for the destruction of tumor without affecting surround tissues. 


\section{FUTURE WORK}

The promising results of the experiments that were performed in the present study to develop Res-PdNPs for prostate cancer treatment and imaging requires further research work in order to transfer them from experimental work to clinical trials.

Further investigation of the oxidized Res products (Res-derived phenols and polyphenols) on the PdNPs are required to fully understand the identity of the encapsulated compounds. The in vivo evaluation results of Res-PdNP-4 in SCID mice showed that these nanoparticles dose response was good, although further studies are required to determine a better dose to see increased efficacy. The dose administrated was well tolerated by the animals, as no animal health problems or discomfort was observed as evidenced by body weight/eating habits of animals. This study was performed through intravenous (IV) administration of the Res-PdNP-4, intraperitoneal (IP) delivery and direct injection into the tumor may show better response as has been the case with many different types of nanoparticles.

The exploration of using an external beam such as near-infrared to excite PdNPs through the use of different laser techniques for photodynamic therapy. More animals studies by using larger animals such as dogs needs to be performed to confirm the efficacy of these nanoparticles to cure prostate cancer because the organs of larger animals such as dogs nearly similar to the organs of humans and therefore the results will be more accurate and similar to what supposed to be in humans. The results of the future studies determine transferring Res-PdNP-4 from experimental trial to clinical trial. The Res-PdNP-4 can be evaluated to treat other cancers such as breast and lung cancer in the future. 


\section{APPENDIX}

A - Cellular viability of Res-PdNP-4 against prostate cancer (PC-3) cells

a)

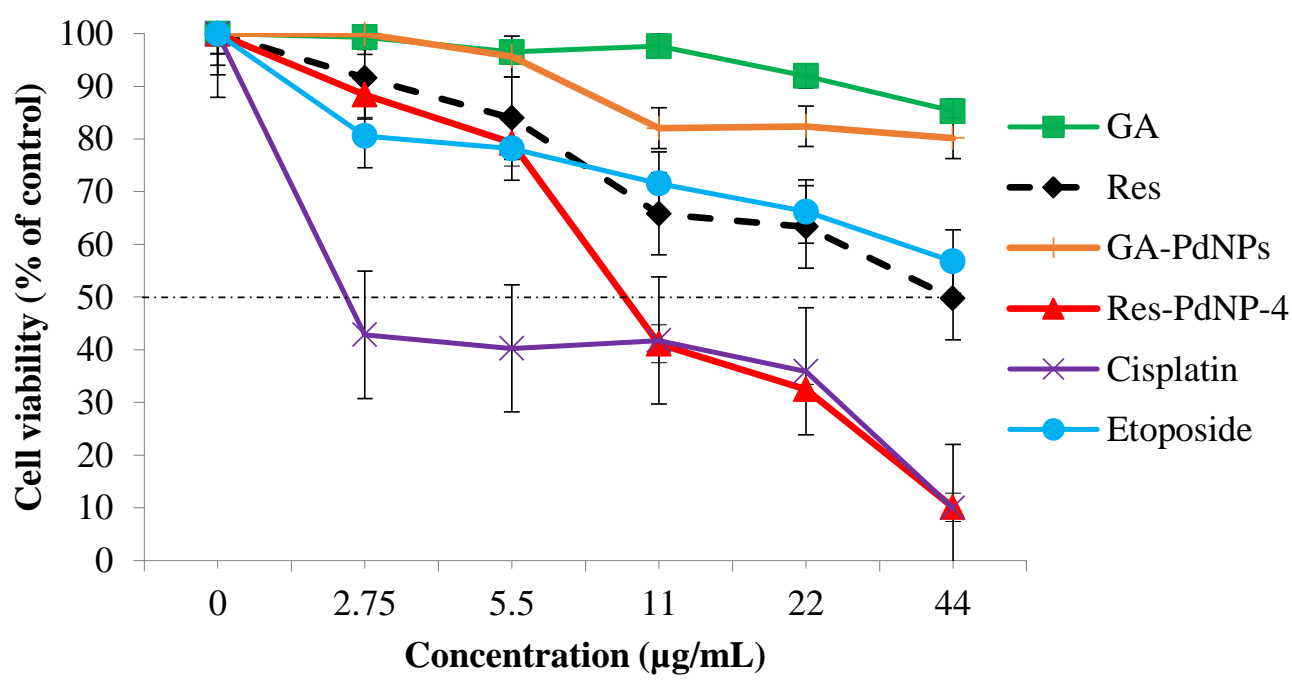

b)

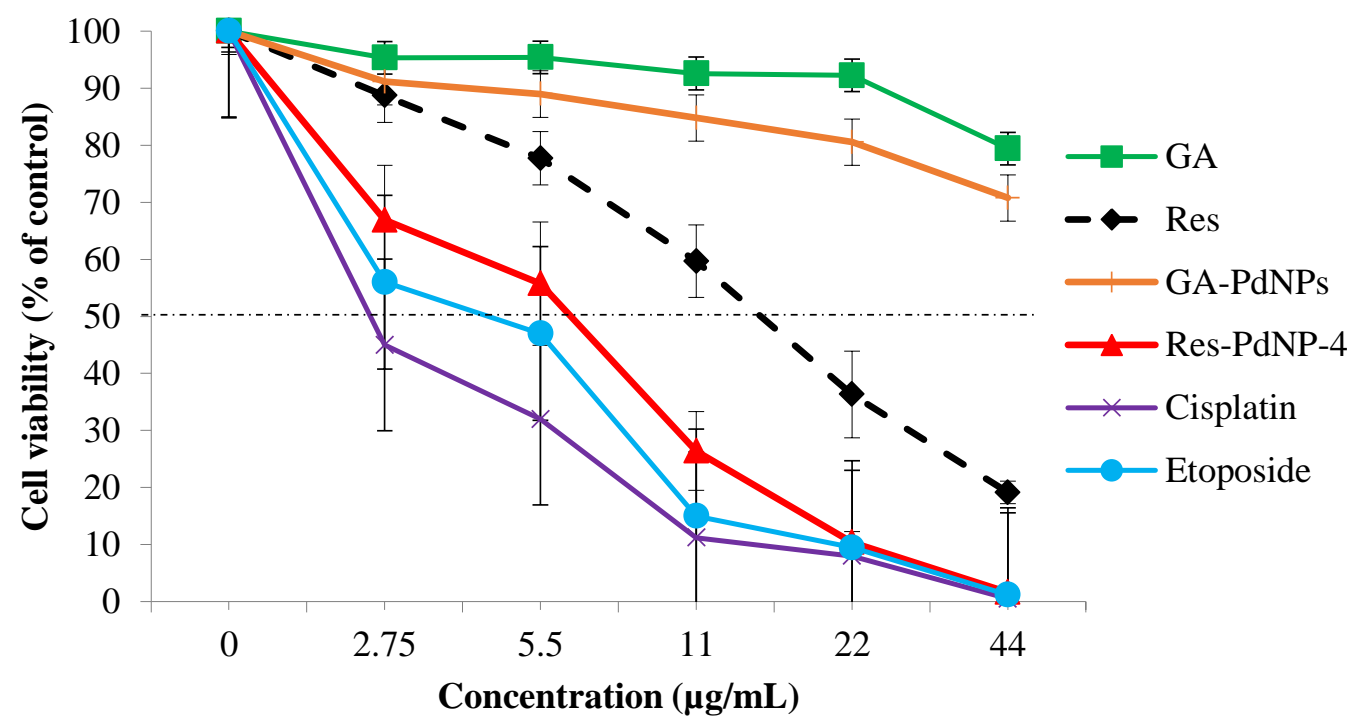

Figure 41: Cell viability of prostate cancer (PC-3) after a) $24 \mathrm{~h}$ and b) $48 \mathrm{~h}$ post incubation with Res-PdNP-4 and controls (free Res, GA, GA-PdNPs, cisplatin and etoposide), n=4; results reported as mean \pm SEM. 
B - Cellular viability of Res-PdNP-4 against human aortic endothelial cells (HAEC)

a)
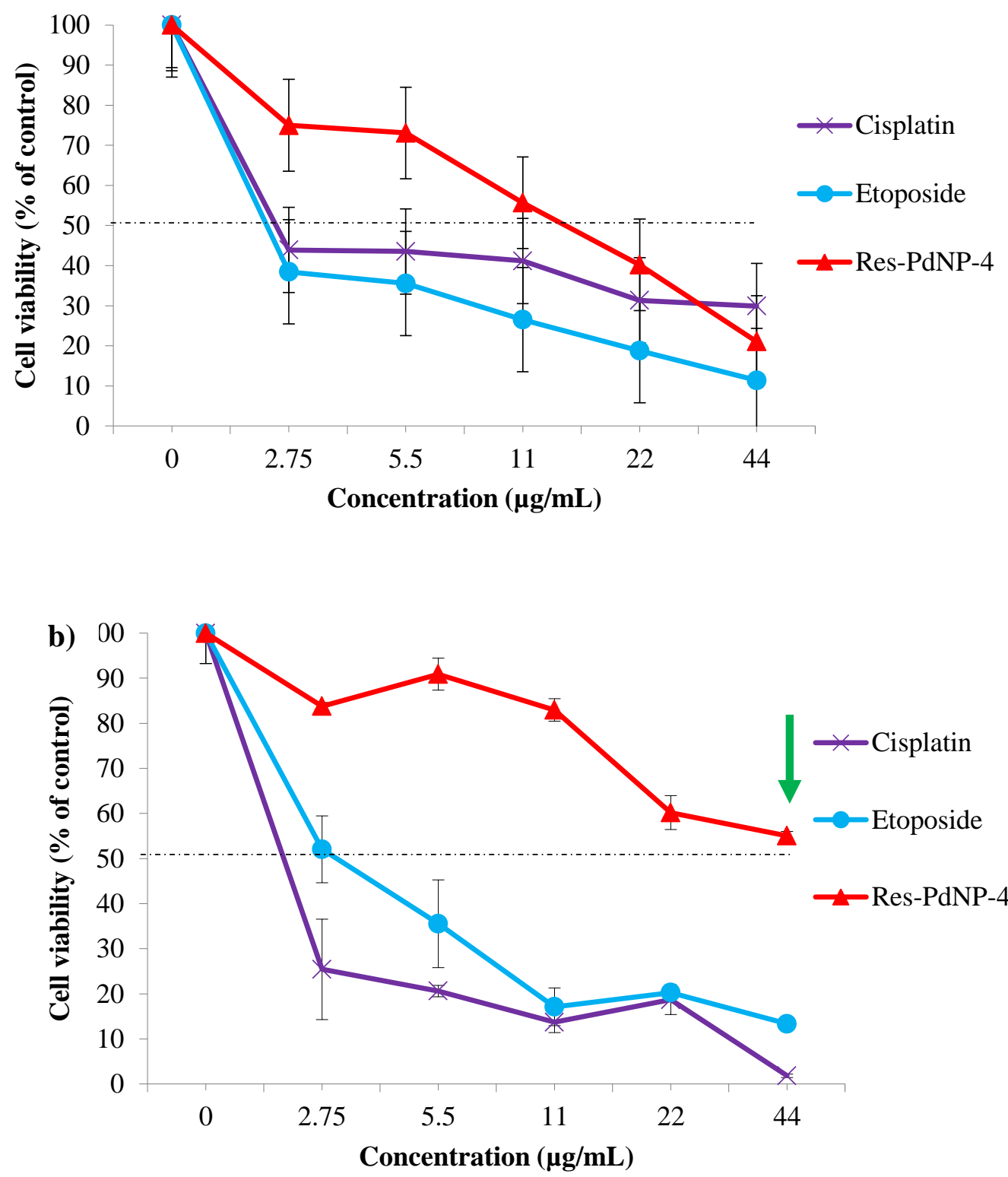

Figure 42: Cell viability of human aortic endothelial cells (HAEC) after a) 24 h and b) 48 h post incubation with Res-PdNP-4 and controls (cisplatin and etoposide). At high ResPdNP-4 concentration no toxicity was observed (green arrow), n=4; results reported as mean \pm SEM. 


\section{REFEENCES}

1. Camont L, Cottart C-H, Rhayem Y, Nivet-Antoine V, Djelidi R, Collin F, Beaudeux JL, Bonnefont-Rousselot D. Simple spectrophotometric assessment of the trans-/cisresveratrol ratio in aqueous solutions. Anal Chim Acta. 2009;634(1):121-128. doi:https://doi.org/10.1016/j.aca.2008.12.003.

2. Nour V, Trandafir I, Muntean C. Ultraviolet Irradiation of Trans-Resveratrol and HPLC Determination of Trans-Resveratrol and Cis-Resveratrol in Romanian Red Wines. $J$ Chromatogr Sci. 2012;50(10):920-927. http://dx.doi.org/10.1093/chromsci/bms091.

3. Allan KE, Lenehan CE, Ellis AV. UV Light Stability of $\alpha$-Cyclodextrin/Resveratrol Host

- Guest Complexes and Isomer Stability at Varying pH. Aust. J. Chem. 2009; 62(8) 921926. doi:10.1071/CH08506.

4. Kora AJ, Rastogi L. Green synthesis of palladium nanoparticles using gum ghatti (Anogeissus latifolia) and its application as an antioxidant and catalyst. Arab J Chem. 2015:0-9. doi:10.1016/j.arabjc.2015.06.024.

5. Shalik MR, Ali ZJ, Khan M, Kuniyil M, Assal ME, Alkathlan HZ, Al-warthan A, Siddqui MR, Khan M, Adil SF. Green Synthesis and Characterization of Palladium Nanoparticles Using Origanum vulgare L. Extract and Their Catalytic Activity. Molecules. 2017;22(1):165. doi:10.3390/molecules22010165.

6. Kalaiselvi A, Roopan SM, Madhumitha G, Ramalingam C, Elango G. Synthesis and characterization of palladium nanoparticles using Catharanthus roseus leaf extract and its 
application in the photo-catalytic degradation. Spectrochim Acta Part A Mol Biomol Spectrosc. 2015;135:116-119. doi:https://doi.org/10.1016/j.saa.2014.07.010.

7. Kattumuri V, Katti K, Bhaskaran S, Boote EJ, Casteel SW, Fent GM, Robertson DJ, Chandrasekhar M, Kannan R, Katti KV. Gum arabic as a phytochemical construct for the stabilization of gold nanoparticles: In vivo pharmacokinetics and X-ray-contrast-imaging studies. Small. 2007;3(2):333-341. doi:10.1002/smll.200600427.

8. Kapoor DN, Bansal A, Sharma R, Dhawan S. Advanced Nanomedicine: Present Contributions and Future Expectations. Am J Phytomedicine Clin Ther. 2013;1(2):124139.

9. Shukla R, Nune SK, Chanda N, Katti K, Mekapothula S, Kulkarni RR, Welshons WV, Kannan R, Katti KV. Soybeans as a phytochemical reservoir for the production and stabilization of biocompatible gold nanoparticles. Small. 2008;4(9):1425-1436. doi:10.1002/smll.200800525.

10. Honary S, Zahir F. Effect of zeta potential on the properties of nano-drug delivery systems - A review (Part 1). Trop J Pharm Res. 2013;12(2):255-264. doi:10.4314/tjpr.v12i2.19.

11. Sikora A, Bartczak D, Geißler D, Kestens V, Roebben G, Ramaye Y, Varga Z, Palmai M, Shard AG, Goenaga-Infante H, Minelli C. A systematic comparison of different techniques to determine the zeta potential of silica nanoparticles in biological medium. Anal Methods. 2015;7(23):9835-9843. doi:10.1039/c5ay02014j.

12. Abreu FOM da S, Silva NA da, Sipauba M de S, Marques TF, Bomfim TA, Monteiro Jr. 
OA, Forte MM. Chitosan and gum arabic nanoparticles for heavy metal adsorption. Polímeros. 2018;28(3):231-238. doi:10.1590/0104-1428.02317.

13. Danaei M, Dehghankhold M, Ataei S, Hasanzadeh DF, Javanmard R, Dokhani A, Khorasani S, Mozafari MR. Impact of Particle Size and Polydispersity Index on the Clinical Applications of Lipidic Nanocarrier Systems. Pharmaceutics. 2018:1-17. doi:10.3390/pharmaceutics10020057.

14. Moreno-Trejo MB, Sánchez-Domínguez M. Mesquite gum as a novel reducing and stabilizing agent for modified tollens synthesis of highly concentrated ag nanoparticles. Materials (Basel). 2016;9(10):14-22. doi:10.3390/ma9100817.

15. Cui H, Webber MJ, Stupp SI. Self-Assembly of Peptide Amphiphiles: From Molecules to. Stud Polit Econ. 2010;94(1):1-18. doi:10.1002/bip.

16. Tianou H, Wang W, Yang X, Cao Z, Kuang Q, Wang Z, Shan Z, Jin M, Yin Y. Inflating hollow nanocrystals through a repeated Kirkendall cavitation process. Nat Commun.:1-8. doi:10.1038/s41467-017-01258-0.

17. Kellermeier M, Gebauer D, Melero-garcía E, Drechsler M, Talmon Y, Kienle L, Colfen H, Garcia-Ruiz JM, Kunz W. Colloidal Stabilization of Calcium Carbonate Prenucleation Clusters with Silica. Adv Funct Mater. 2012:4301-4311. doi:10.1002/adfm.201200953.

18. Yasuda H. Microsecond time- and subnanometer spatial-scale in situ observations of crystallization process in amorphous antimony nanoparticles by the UHVEM newly developed at Osaka University. EMC. 2016; 1:3. 
doi:10.1002/9783527808465.EMC2016.6125.

19. Thanh NTK, Maclean N, Mahiddine S. Mechanisms of Nucleation and Growth of Nanoparticles in Solution. Chem Rev. 2014;114(15):7610-7630. doi:10.1021/cr400544s.

20. Vollath D, Fischer FD, Holec D. Surface energy of nanoparticles - influence of particle size and structure. Beilstein J. Nanotechnol. 2018:2265-2276. doi:10.3762/bjnano.9.211.

21. Grammatikopoulos P, Cassidy C, Singh V, Sowwan M. Coalescence-induced crystallisation wave in Pd nanoparticles. Scientific Reports. 2014:1-9. doi:10.1038/srep05779.

22. De D, Toulouse DE. Doctorat de l' université de toulouse. 2015:1-190.

23. Khazaei A, Rahmati S, Hekmatian Z, Saeednia S. A green approach for the synthesis of palladium nanoparticles supported on pectin: Application as a catalyst for solvent-free Mizoroki - Heck reaction. Journal Mol Catal A, Chem. 2013;372:160-166. doi:10.1016/j.molcata.2013.02.023.

24. Castaño A, Maurer MS. Natural history and therapy of TTR-cardiac amyloidosis: emerging disease-modifying therapies from organ transplantation to stabilizer and silencer drugs.. Heart Fail Rev. 2015;20(2):163-178. doi:10.1007/s10741-014-9462-7.

25. Gamal-Eldeen AM, Moustafa D, El-Daly SM, El-Hussieny EA, Saleh S, Khoobchandani M, Bacon KL, Gupta S, Katti K, Shukla R, Katti KV. Photothermal therapy mediated by gum Arabic-conjugated gold nanoparticles suppresses liver preneoplastic lesions in mice. J Photochem Photobiol B Biol. 2016;163:47-56. doi:10.1016/j.jphotobiol.2016.08.009. 
26. Gamal-Eldeen AM, Moustafa D, El-Daly SM, Abo-Zeid MAM, Saleh S, Khoobchandani M, Katti K, Shukla R, Katti KV. Gum Arabic-encapsulated gold nanoparticles for a noninvasive photothermal ablation of lung tumor in mice. Biomed Pharmacother. 2017;89:1045-1054. doi:10.1016/j.biopha.2017.03.006.

27. Fent GM, Casteel SW, Kim Y, Kannan R, Katti K, Chanda N, Katti K. Biodistribution of maltose and gum arabic hybrid gold nanoparticles after intravenous injection in juvenile swine. Nanomedicine Nanotechnology, Biol Med. 2009;5(2):128-135. doi:10.1016/j.nano.2009.01.007.

28. Ribeiro De Barros H, Cardoso MB, Camargo De Oliveira C, Franco CR, de Lima Belan S, Vidotti M, Reigel-Vidotti IC. Stability of gum Arabic-gold nanoparticles in physiological simulated pHs and their selective effect on cell lines. RSC Adv. 2016;6(12):9411-9420. doi:10.1039/c5ra24858b.

29. Wu W, Kong X, Zhang C, Hua Y, Chen Y. Improving the stability of wheat gliadin nanoparticles - Effect of gum arabic addition. Food Hydrocoll. 2018;80:78-87. doi:10.1016/j.foodhyd.2018.01.042.

30. Williams DN, Gold KA, Holoman TRP, Ehrman SH, Wilson OC. Surface modification of magnetic nanoparticles using gum arabic. J Nanoparticle Res. 2006;8(5):749-753. doi:10.1007/s11051-006-9084-7.

31. Al-jumaily EFA, Hamid GS, Ali KF. Synthesis and Total Phenol Content of New Resveratrol Derivative. Am J Adv Drug Deliv. 2014;2(3):320-329. 
32. Bonechi C, Martini S, Ciani L, Lamponi S, Rebmann H, Rossi C, Ristori S. Using liposomes as carriers for polyphenolic compounds: The case of Trans-resveratrol. PLoS One. 2012;7(8). doi:10.1371/journal.pone.0041438.

33. Daoub RMA, Elmubarak AH, Misran M, Hassan EA, Osman ME. Characterization and functional properties of some natural Acacia gums. J Saudi Soc Agric Sci. 2016. doi:10.1016/j.jssas.2016.05.002.

34. Nep EI, Conway BR. Characterization of Grewia Gum, a Potential Pharmaceutical Excipient. $\quad J \quad$ Excipients Food Chem. 2010;1(1):30-40. https://ojs.abo.fi/index.php/jefc/article/view/12.

35. Sarika PR, Nirmala RJ. Curcumin loaded gum Arabic aldehyde-gelatin nanogels for breast cancer therapy. Mater Sci Eng C. 2016;65:331-337. doi:10.1016/j.msec.2016.04.044.

36. Lu DL, Ding DJ, Yan WJ, Li RR, Dai F, Wang Q, Yu SS, Li Y, Jin XL, Zhou B. Influence of glucuronidation and reduction modifications of resveratrol on its biological activities. ChemBioChem. 2013;14(9):1094-1104. doi:10.1002/cbic.201300080.

37. Daoub RMA, Elmubarak AH, Misran M, Hassan EA, Osman ME. Characterization and functional properties of some natural Acacia gums. J Saudi Soc Agric Sci. 2016. doi:10.1016/j.jssas.2016.05.002.

38. Singh S, Bothara SB. Physico-chemical and structural characterization of mucilage isolated from seeds of Diospyros melonoxylon Roxb. Brazilian J Pharm Sci. 2014;50(4):713-726. doi:10.1590/S1984-82502014000400006. 
39. Fajardo AR, Fávaro SL, Rubira AF, Muniz EC. Dual-network hydrogels based on chemically and physically crosslinked chitosan/chondroitin sulfate. React Funct Polym. 2013;73(12):1662-1671. doi:10.1016/j.reactfunctpolym.2013.10.003.

40. Camont L, Collin F, Couturier M, Thérond P, Jore D, Gardès-Albert M, BonnefontRousselot D. Radical-induced oxidation of trans-resveratrol. Biochimie. 2012;94(3):741747. doi:10.1016/j.biochi.2011.11.005.

41. Palliyarayil A, Jayakumar KK, Sil A, Kumar NS. A Facile Green Tea Assisted Synthesis of Palladium Nanoparticles Using Recovered Palladium from Spent Palladium Impregnated Carbon. Johnson Matthey Technol. Rev. 2018, 62, (1), 60-73.

42. Queiroz AN, Gomes BAQ, Moraes Jr. WM, Borges RS. A theoretical antioxidant pharmacophore for resveratrol. Eur. J. Med. Chem. 2009;44(4):1644-1649. doi.org/10.1016/j.ejmech.2008.09.023.

43. Corduneanu O, Janeiro P, Brett AMO. On the Electrochemical Oxidation of Resveratrol. Electroanalysis. 2006;18(8):757-762.

44. Bao LL, Liu ZQ. Hybrid of Resveratrol and Glucosamine: An Approach To Enhance Antioxidant Effect against DNA Oxidation. Chem. Res. Toxicol. 2018;31:936-944. doi. 10.1021/acs.chemrestox.8b00136.

45. Keylor MH, Matsuura BS, Stephenson CRJ. Chemistry and Biology of ResveratrolDerived Natural Products. Chem. Rev. 2015;115:8976-9027. doi.10.1021/cr500689b.

46. Khoobchandani M, Zambre A, Katti K, Lin C, Katti K V. Green Nanotechnology from 
Brassicaceae: Development of Broccoli Phytochemicals - Encapsulated Gold Nanoparticles and Their Applications in Nanomedicine. Int J Nanotechnol Nanomed. 2013. doi:10.1177/1943089213509474.

47. Shukla R, Chanda N, Zambre A, Upendran A, Katti K, Kulkarni RR, Nune SK, Casteel SW, Smith CJ, Vimal J, Boote E, Robertson JD, Kan P, Engelbrecht H, Watkinson LD, Carmack TL, Lever RR, Cutler CS, Caldwell C, Kannan R, Katti KV. Laminin receptor specific therapeutic gold nanoparticles (198AuNP-EGCg) show efficacy in treating prostate cancer. Proc Natl Acad Sci. 2012;109(31):12426-12431. doi:10.1073/pnas.1121174109.

48. Khoobchandani M, Katti K, Maxwell A, Fay WP, Katti K V. Laminin receptor-avid nanotherapeutic EGCg-AuNPs as a potential alternative therapeutic approach to prevent restenosis. Int J Mol Sci. 2016;17(3). doi:10.3390/ijms17030316.

49. Shi Q, Wada K, Ohkoshi E, Lin L, Huang R, Morris-Natschke SL, Goto M, Lee KH. Antitumor agents 290. Design, synthesis, and biological evaluation of new LNCaP and PC-3 cytotoxic curcumin analogs conjugated with anti-androgens. Bioorganic Med Chem. 2012;20(13):4020-4031. doi:10.1016/j.bmc.2012.05.011.

50. Zhang L, Chen X, Wu J, Ding S, Wang X, Lei Q, Fang W. Palladium nanoparticles induce autophagy and autophagic flux blockade in Hela cells. RSC Adv. 2018;8(8):4130-4141. doi:10.1039/c7ra11400a.

51. Foroozandeh P, Aziz AA. Insight into Cellular Uptake and Intracellular Trafficking of Nanoparticles. Nanoscale Res Lett. 2018;13. doi:10.1186/s11671-018-2728-6. 
52. Tiwari PM, Eroglu E, Bawage SS, Vig K, Miller ME, Pillai S, Dennis VA, Singh SR. Enhanced intracellular translocation and biodistribution of gold nanoparticles functionalized with a cell-penetrating peptide (VG-21) from vesicular stomatitis virus. Biomaterials. 2014;35(35):9484-9494. doi:10.1016/j.biomaterials.2014.07.032.

53. Bagherifam S, Skjeldal FM, Griffiths G, Maelandsmo GM, Engebraten O, Nystrom B, Hasirci V, Hasiri N. PH-responsive Nano carriers for doxorubicin delivery. Pharm Res. 2015;32(4):1249-1263. doi:10.1007/s11095-014-1530-0.

54. Khoobchandani M, Katti K, Zambre A, Katti K V. Cellular uptake and cytotoxic effects of broccoli phytochemicals based gold nanoparticles (B-AuNPs): Enhanced cancer therapeutic efficacy. Tech Proc 2013 NSTI Nanotechnol Conf Expo, NSTI-Nanotech 2013. $2013 ; 3: 422-425$.

55. Yang C, Uertz J, Yohan D, Chithrani BD. Peptide modified gold nanoparticles for improved cellular uptake, nuclear transport, and intracellular retention. Nanoscale. 2014;6(20):12026-12033. doi:10.1039/c4nr02535k.

56. Diaz-Quiñones A, Figueiredo ML, Shearer JJ, Neto MF, Umbaugh CS. A dock derived compound against laminin receptor (37 LR) exhibits anti-cancer properties in a prostate cancer cell line model. Oncotarget. 2017;9(5):5958-5978. doi:10.18632/oncotarget.23236.

57. Sarkis M, Ghanem E, Rahme K. Jumping on the Bandwagon: A Review on the Versatile Applications of Gold Nanostructures in Prostate Cancer. Int J Mol Sci. 2019;20(4):970. doi:10.3390/ijms20040970. 
58. Mortensen A, Aguilar F, Crebelli R, Domenico AD, Frutos MJ, Galtier P, Gott D, Gundert-Remy U, Lambre C, Leblanc JC, Lindtner O, Moldeus P, Mosesso P, Oskarsson A, Parent-Massin D, Stankovic I, Waalkens-Berendsen I, Woutersen RA, Wright M, Younes M, Brimer L, Christodoulidou A, Lodi F, Tard A, Dusemund B. Re- evaluation of acacia gum (E 414) as a food additive. EFSA J. 2017;15(4):1-51. doi:10.2903/j.efsa.2017.4741.

59. Ali BH, Ziada A, Blunden G. Biological effects of gum arabic: A review of some recent research. Food Chem Toxicol. 2009;47(1):1-8. doi:10.1016/j.fct.2008.07.001.

60. Patel S, Goyal A. Applications of natural polymer gum Arabic: A review. Int J Food Prop. 2015;18(5):986-998. doi:10.1080/10942912.2013.809541.

61. Kannan R, Rahing V, Cutler C, Pandrapragada R, Katti KK, Kattumuri V, Robertson JD, Casteel SJ, Jurisson S, Smith C, Booe E, Katti KV. Nanocompatible Chemistry toward Fabrication of Target-Specific Gold Nanoparticles. J. Am. Chem. Soc. 2006:11342-11343. doi:10.1021/ja063280c.

62. Axiak-Bechtel SM, Upendran A, Lattimer JC, Kelsey J, Cutler CS, Selting KA, Bryan JN, Henry CJ, Boote E, Tate DJ, Bryan ME, Katti KV, Kannan R. Gum arabic-coated radioactive gold nanoparticles cause no short-term local or systemic toxicity in the clinically relevant canine model of prostate cancer. Int J Nanomedicine. 2014;28(9):500111. doi:10.2147/IJN.S67333.

63. Mansouri-Torshizi H, Rezaei E, Kamranfar F, Majd MH. Investigating the apoptosis ability of ethylenediamine 8-hydroxyquinolinato palladium (II) complex. Adv Pharm Bull. 
2016;6(3):449-453. doi:10.15171/apb.2016.058.

64. Ulukaya E, Frame FM, Cevatemre B, Pellacani D, Walker H, Mann VM, Simms MS, Stower MJ, Yilmaz VT, Maitland NJ. Differential Cytotoxic Activity of a Novel Palladium-Based Compound on Prostate Cell Lines, Primary Prostate Epithelial Cells and Prostate Stem Cells. PLoS One. 2013;8(5). doi:10.1371/journal.pone.0064278.

65. Hadizadeh S, Najafzadeh N, Mazani M, Amani M, Mansouri-Torshizi H, Niapour A. Cytotoxic Effects of Newly Synthesized Palladium(II) Complexes of Diethyldithiocarbamate on Gastrointestinal Cancer Cell Lines. Biochem Res Int. 2014;2014. doi:10.1155/2014/813457.

66. Chen M, Chen S, He C, Mo, S, Wang X, Liu G, Zheng N. Safety profile of twodimensional Pd nanosheets for photothermal therapy and photoacoustic imaging. Nano Research. 2016;10(4):1234-1248. doi 10.1007/s12274-016-1349-6

67. Yu YX, Xu S, You H, Zhang Y, Yang B, Sun X, Yang L, Chen Y, Fu S, Wu J. In vivo synergistic anti-tumor effect of Paclitaxel nanoparticles combined with radiotherapy on human cervical carcinoma. Drug Deliv. 2017;24(1):75-82. doi:10.1080/10717544.2016.1230902. 


\section{VITA}

Velaphi Clement Thipe was born in South Africa. He received his Diploma degree in Biotechnology from the Department of Biotechnology from the University of Johannesburg (UJ), South Africa. Immediately, after his graduation, he enrolled for a Bachelor of Technology (BTech) in Biotechnology from UJ. Velaphi obtained his MSc degree in Nanoscience from the Department of Applied Chemistry from UJ sponsored by the Department of Science and Technology. After he earned the Master degree, he worked for the South African Breweries as a brewing technician operator. Then he got a Fulbright scholarship from United States Department of State and South Africa's National Research Foundation (NRF) to pursue PhD degree in Chemistry at the University of Missouri-Columbia. His $\mathrm{PhD}$ research is directed towards developing new protocols to produced high resveratrol corona palladium nanoparticles to be used

for the treatment of prostate cancer. Velaphi is planning to graduate from the University of Missouri in May of 2019. Then, he will go back home and possibly do a joint postdoctoral appointment between the Nuclear and Energy Research Institute (IPEN) in Brazil and the Institute of Green Nanotechnology at the University of Missouri-Columbia. 